\title{
Multi-objective Design of a Transonic \\ Turbocharger Compressor with Reduced Noise and Increased Efficiency
}

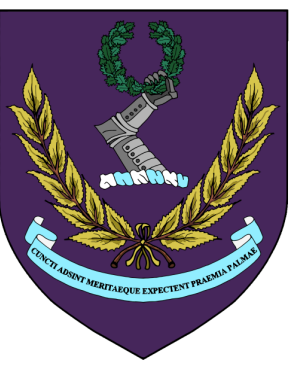

Peng Wang

Department of Mechanical Engineering University College London

A thesis submitted for the degree of

Doctor of Philosophy

April, 2017 
I would like to dedicate this thesis to my families and friends ... 


\section{Declaration}

I, Peng Wang, confirm that the work presented in this thesis is my own. Where information has been derived from other sources, I confirm that this has been indicated in the thesis.

Signature: 王鹏

Name: Peng Wang

Date: April 11, 2017 


\section{Acknowledgements}

It would not be possible to complete this work without the countless help and support from many people around me, and it is only possible to give particular thanks to some of them here.

This thesis will never be produced without the help, support and guidance from my principal supervisor, Prof Mehrdad Zangeneh, who introduced me to the field of turbomachinery aerodynamics, aeroacousics, and optimisations. After three years and a half of doing my $\mathrm{PhD}$, I had the privilege to get a job offer from the company Advanced Design Technology, Ltd founded by him even before the completion of all $\mathrm{PhD}$ work. It has been almost three years since I started to work at ADT, and when I look back the journey I have made, I realise how fruitful this experience has been. At ADT, I have had the opportunities to maximise and use the skills and knowledge I learned through the course of this $\mathrm{PhD}$. I have greatly broadened my horizon and deepened my knowledge of the academic research and industry applications in this fascinatingly interesting and challenging field. I am also one of those very lucky people who can have the chance to work with the world-class leading companies for turbomachine's manufacturing and design. I have learned a great deal from them. None of these could happen if I was not admitted by my supervisor in 2010 when I was struggling for finding a job in the very crucial market. So, thank you very much professor.

I must acknowledge the financial, academic, and technical support from Napier turbocharger Ltd. My both supervisors Dr Frank Heyes and Dr Paul Roach have been extremely patient with my research. They provided invaluable advices for my work and facilitated good 
experimental measurements for my final impeller design. I also received very generous hospitality from them every time when I visited Napier for discussions. To them, I express my sincere gratitude.

Experiences at UCL mechanical engineering department will be undoubtedly the most important time for my academic career. I have spent ten years in this place where I have had great opportunities to work with many intelligent and gifted academics. Prof Nader Saffri, who served as my second supervisor, provided me with significant moral support and careful advices through the period of my $\mathrm{PhD}$. Dr William Suen, who was my undergraduate personal tutor and our graduate student tutor, has given me massive help and support ever since I joined this department in 2006 as a first year undergraduate. I still remember that day when I first stepped into his office. Dr Rama Balachandran, who acted as my third year project supervisor, is a very important model for me to follow in pursuing the academic excellence. He is the one who firstly introduced me to the field of CFD and aerothermal acoustics. The experience with him of doing the third year project on the CFD simulations for the sheared layer aerodynamics in a combustion chamber actually laid the necessary foundation for my $\mathrm{PhD}$ research, and I can only realise the importance until I look back now. Dr Alvin Blackie, who retired three years ago and was the deputy head of department and undergraduate tutor, has given me plenty of help and advices ever since I joined this institute. Particularly, I received a very kind reference letter from him to support my $\mathrm{PhD}$ application in 2010. I am rather grateful that he invited me to teach the first year computing course which proved to me a really useful experience.

I am a very lucky man who has had opportunities to work with many fellow PhD students. I must give special thanks to my group members, Pietro Boselli, Jiangnan Zhang, Jeremy Nahon and Irfan Muhammad. Pietro, you are the one I envy most for your smartness and huge muscles. Jiangnan, I have mentioned to many of our common friends that 
you are the one I admire a lot because of your ability to concentrate regardless of any surrounding environment. Fellows from other research groups such as Liu Liu and Ye Yuan, I am glad we had the chance to share a flat. Yajie Li, Qicheng Meng, Chongwei Zhang, Guodong Xu, Anne Charlotte Gaillard, I had the most interesting discussions with you guys about many relevant and irrelevant things for academic research. Those joyful moments have been imprinted in my mind forever. Taaha Hussain, Mart Magi, Baptiste Duboc, you are my oldest friends at UCL since the undergraduate time. Zhiyu Yuan, Yuanchang Liu, Rui Song, Wenwen Liu, Xujiang Wang, Yang Chen, Cedrick Lin, thank all of you very much for the wonderful times in Roberts building, Gower Street, Hampstead Road, and many other places.

ADT's staffs are inseparable from the completion of this work either. Our journey still keeps going on. I need to give my special thanks to Fabio Pengue and Mathieu de Maillard, who were the key design engineers at ADT, and they taught me how to use TD1 properly and showed me the design process step by step. Richard Hunsley, who was our engineering team manager, has been the example for my career development. Nunzio, Abul and Raoul, I cannot remember how many laughters we have shared with. Pedro, David, Peixin, Faidon, Darshan and Paolo for you guys all, I offer my sincere gratitude too. We will keep adventuring as ADT staffs with great fun towards our next big goal!

Last but not least, I would like to thank my family for all their love and encouragement. My parents, Juncai Wang and Yongfang Chen who raised me with a mind full of curiosities. Even if they received little school education, they have never appeared to be short-sighted at all. About 12 years ago, their decision to send me to study abroad has changed my life entirely. Their unquestionable support and love can never be over-exaggerated. And most of all to my beautiful, loving, supportive, encouraging, and patient girlfriend Jing Qian whose 
faithful support during the entire course of this $\mathrm{PhD}$ is so appreciated. Thank you, my love! You have shown magnificent braveness during these years when I am not with you in China. 


\begin{abstract}
In marine diesel engines, transonic centrifugal compressors are widely used due to their capabilities to: 1) downsize engines by increasing output power; 2) cause less fuel consumption; 3) enhance the combustion efficiency. The design of a high performance transonic compressor still poses a great challenge to the turbomachinery community. Apart from the traditional requirements such as good choke and stall margin, high boosting pressure ratio, and high stage efficiency, it is also necessary to reduce the turbocharger noise as much as possible for a more comfortable working environment. The most effective way is to reduce the compressor noise at the source, i.e. the compressor impeller itself must produce less aerodynamic noise rather than using any silencers. This thesis is dedicated to present a design methodology for such multi-disciplinary and multi-objective tasks, and propose a new impeller design for a marine turbocharger.

The conventional turbomachinery design method has almost reached its bottleneck even if it is coupled with advanced optimisation algorithms. A different design philosophy, which is known as inverse design theory, suggests an alternative solution for such design challenges. It provides a different perspective to look at the problem. Rather than starting with the blade profiling, this approach prescribes the flow field on the blade surface and robustly finds the corresponding blade geometry that would result in the given flow field. Since the flow field plays a predominant role in determining both aerodynamic and aeroacoustic performances, it gives designers more direct control for getting an optimal design. Thus, this method can significantly strengthen the performances in a much shortened design time.
\end{abstract}


An in-house aeroacoustic code has been developed based upon the well-known Ffowcs-Williams and Hawkings equation to predict the rotor induced tonal noise at the blade passing frequency and its harmonics. It can also be easily expanded to predict the transient flow induced broadband noise if the very fine unsteady calculations are available.

A new centrifugal compressor impeller was designed in this dissertation using the inverse design code TURBOdesign1. Its performances are evaluated from aerodynamic, aeroacoustic and mechanical aspects. The CFD simulations for the compressor characteristics and internal flow field details, the numerical predictions of aeroacoustic sound emissions, and FEA analysis for the structure integrity have all been attempted to thoroughly assess the performances of baseline and optimised impellers. The computational results found that the new impeller can lead to better performances in all three aspects, which are supported by the experimental measurements conducted for both impellers using the same test configurations. The experimental data confirmed that the inversely redesigned impeller wheel provide a wider compressor operating range, higher efficiency at large rotating speed, and a few $\mathrm{dB}(\mathrm{A}) \mathrm{s}$ lower noise emissions in the upstream.

The present work suggests that the synergistic use of the inverse design approach, aeroacoustic solver, CFD simulations and FEA analysis can allow designers to effectively carry out the multi-disciplinary and multi-objective designs for turbomachines. 


\section{Contents}

Contents ix

List of Figures $\quad$ xiv

List of Tables $\quad$ xxiii

Nomenclature $\quad$ xxiv

1 Introduction: design challenges of a transonic turbocharger compressor 1

1.1 Research background . . . . . . . . . . . . . . . 1

1.2 Research objectives . . . . . . . . . . . . . . . . . . . 4

1.3 Thesis structure . . . . . . . . . . . . . . . 5

2 Literature survey: multi-objective design and analysis for a tran$\begin{array}{ll}\text { sonic centrifugal compressor } & 7\end{array}$

2.1 Compressor stage components . . . . . . . . . . . . 8

2.2 Classifications of the turbocharger noise . . . . . . . . . . 10

2.3 Theory of aerodynamic sound: aeroacoustics . . . . . . . . . 13

2.3.1 Pre-acoustic analogy era: rotor noise physics . . . . . . . . 13

2.3.2 Acoustic analogy era: Lighthill and FW-H . . . . . . . . . 14

2.3.3 Recent research on the aerodynamic noise prediction approach: an integration of FW-H and CFD . . . . . . . . 15

2.4 Turbomachinery aeroacoustics . . . . . . . . . . . . . . 16

2.4.1 Turbomachinery noise sources . . . . . . . . . . . 16 
2.4.2 Numerical studies on compressor noise . . . . . . . . . . 20

2.5 Inverse design approach for turbomachines . . . . . . . . . . . . 21

2.5.1 Potential flow method . . . . . . . . . . . . 22

2.5.2 Circulation method . . . . . . . . . . . . . 22

2.5.3 Time marching method . . . . . . . . . . . . . . 23

2.5.3.1 $\Delta P \operatorname{method} \ldots \ldots \ldots \ldots 24$

2.5.3.2 Swirl velocity method . . . . . . . . . 25

2.5.4 Summary . . . . . . . . . . . . . . . 26

2.6 Existing studies of inversely designed turbomachines with acoustic optimisations . . . . . . . . . . . . . . 26

2.7 Summary . . . . . . . . . . . . . . . . . . . . 27

3 Mathematical formulations for aeroacoustics $\quad 28$

3.1 Lighthill's acoustic analogy . . . . . . . . . . . . . . 28

3.2 Curle's equation . . . . . . . . . . . . . . . . . . . 30

3.3 Ffowcs-Willams and Hawkings equation: a convective scheme . . . 31

3.3.1 Convective FW-H . . . . . . . . . . . . . . . . . . . 32

3.4 Integral formulations . . . . . . . . . . . . . . . . . 36

3.5 Summary . . . . . . . . . . . . . . . . . . . . . 38

4 Aeroacoustic solver for rotor-induced noise 39

4.1 Integral formulations . . . . . . . . . . . . . . . . . . . . 39

4.2 Integral solution to the convective $\mathrm{FW}-\mathrm{H}$ equation . . . . . . . . 41

4.2 .1 Retarded-time algorithm . . . . . . . . . . . . . 43

4.2.1.1 Thickness noise . . . . . . . . . . . . . . 43

4.2.1.2 Loading noise . . . . . . . . . . . . . . . . . 45

4.2 .2 Mid-panel quadrature . . . . . . . . . . . . . . . . . . . 46

4.2 .3 Root-find algorithm . . . . . . . . . . . . . . . 47

4.2.4 Drawbacks of retarded-time algorithm . . . . . . . . 49

4.2.4.1 Source-time dominant algorithm . . . . . . . . 49

4.2.5 Collapsing sphere algorithms . . . . . . . . . . . . 50

4.2.6 Emission surface algorithms . . . . . . . . . . . . 51

4.2.7 Permeable surface integration scheme . . . . . . . . . . 52 
4.3 Summary . . . . . . . . . . . . . . . . 54

5 Applications and validations of the developed aeroacoustic solver 56

5.1 Coding algorithm development . . . . . . . . . . . . . 57

5.2 UH1H NACA 0012 helicopter rotor . . . . . . . . . . . . 61

5.2.1 Geometry definition . . . . . . . . . . . . 61

5.2 .2 Experimental set-up . . . . . . . . . . . . 62

5.3 Numerical results of UH1H NACA 0012 rotor noise . . . . . . . . 62

5.4 Transonic centrifugal compressors . . . . . . . . . . . . 69

5.4 Test facility . . . . . . . . . . . . . . . 70

5.4 .2 Validation ................... . . 70

5.4 .3 CFD analysis . . . . . . . . . . . . . . . 74

6 Mathematical formulations of the inverse design method 83

6.1 Theoretical formulation of inverse design . . . . . . . . . . . . . . 84

6.1.1 Blade loading . . . . . . . . . . . . . . . 86

6.1.2 Flow solver: vorticity method and Clebsch formulation . . 90

6.1.2.1 Calculation of the density . . . . . . . . 90

6.1.2.2 Calculation of the velocity - approximate method 91

6.1.2.3 Calculation of the velocity - exact method . . . . 99

6.1.3 Blade shape calculation . . . . . . . . . . . . . . 101

6.2 Parametrisation of the blade loading . . . . . . . . . . . 102

$6.2 .1 r V_{\theta}^{*}$ at LE and TE . . . . . . . . . . . . . . . 103

$6.2 .2 \partial r V_{\theta}^{*} / \partial m^{*}$ from the inlet to outlet . . . . . . . . . . . 104

6.3 Summary . . . . . . . . . . . . . . . . 106

$\begin{array}{lll}7 & \text { Design and analysis of a new transonic centrifugal impeller } & 108\end{array}$

7.1 Analysis of the existing compressor stage . . . . . . . . . . . 109

7.1.1 Geometry details of baseline impeller . . . . . . . . . . . . 110

7.1.2 CFD analysis for the existing compressor stage . . . . . . . 110

7.1.2.1 Grid independence study . . . . . . . . . . . 110

7.1.2.2 Validation with test results . . . . . . . . . 113

7.2 Design of the new impeller using TURBOdesign1 . . . . . . . 115

7.2 .1 Design point selection . . . . . . . . . . . . 116 
7.2.2 Meridional contour and number of blades . . . . . . . . . . 117

7.2.3 Thickness distribution . . . . . . . . . . . . . . . . . . 117

7.2 .4 Inlet boundary conditions . . . . . . . . . . . . . 118

7.2.5 Blockage factor . . . . . . . . . . . . . . . . 118

7.2.6 Blade loading . . . . . . . . . . . . . . . . . . . . 121

7.2.6.1 Spanwise $r V_{\theta}^{*} \ldots \ldots \ldots . \ldots . \ldots 121$

7.2.6.2 Streamwise $\partial\left(r V_{\theta}^{*}\right) / \partial m^{*} \ldots \ldots \ldots . \ldots 122$

7.2 .7 Stacking . . . . . . . . . . . . . . 126

7.3 Study of impeller structure integrity . . . . . . . . . . . . . . 127

7.4 Performance comparisons at the peak speed . . . . . . . . . 138

7.4.1 Internal flow field details . . . . . . . . . . . . . . . . . 138

7.4.2 Near field acoustics: numerical calculations . . . . . . . . . 139

7.4 .3 Summary . . . . . . . . . . . . . . . . . . . . . 144

7.5 Predicted performances of the complete stage of the redesign . . . 149

7.6 Summary . . . . . . . . . . . . . . . . . . . . 150

8 Evaluations of the inversely designed impeller 152

8.1 Test rig . . . . . . . . . . . . . . . . . . . 153

8.2 Validation of CFD predictions . . . . . . . . . . . . 153

8.3 Measurements of redesigned compressor stage maps . . . . . . . . 155

8.4 Measured acoustic noise . . . . . . . . . . . . . . . 156

8.5 Comparisons of flow physics . . . . . . . . . . . . . . 170

8.5.1 Choke margins . . . . . . . . . . . . . . . . 170

8.5.2 Tip leakage flow . . . . . . . . . . . . . . . 171

8.5.3 Flow separations . . . . . . . . . . . . . . 172

8.5.4 Exit flow uniformity . . . . . . . . . . . . . . 174

8.5.5 Shock wave structures . . . . . . . . . . . . . . 175

8.5.6 Flow in the vaned diffuser . . . . . . . . . . . . . . . . 189

8.5.7 Stall margins . . . . . . . . . . . . . . . . . . . . 191

8.6 Summary . . . . . . . . . . . . . . . . . . 192

9 Conclusions and Future work 195

9.1 Accomplishments achieved in this thesis . . . . . . . . . . 196 
9.2 Recommendation of future work . . . . . . . . . . . . . 196

9.2.1 Aeroacoustics . . . . . . . . . . . . . . 197

9.2.2 Aerodynamics . . . . . . . . . . . . . . . . 198

9.2.3 Turbomachinery design and optimisation . . . . . . . . . 198

$\begin{array}{ll}\text { Bibliography } & 200\end{array}$

$\begin{array}{lr}\text { APPENDICES } & 210\end{array}$

Appendix A Appendix A: Derivation of Formulation 1C in terms $\begin{array}{ll}\text { of source-time derivative } & 211\end{array}$ 


\section{List of Figures}

1.1 Schematic of a turbocharger cycle . . . . . . . . . . . . 2

2.1 Meridional view of a centrifugal compressor stage . . . . . . . . 11

2.2 Noise spectrum of a transonic fan, Cumpsty [1989] . . . . . . . . 17

3.1 Flow over a rigid body whose surface is defined by $f(\mathbf{x}, t) \ldots 33$

4.1 Schematics of collapsing sphere and $\Gamma$ curve . . . . . . . . . . . 42

4.2 Roots of the retarded time equation . . . . . . . . . . . . . . 48

5.1 Flow chart of FW-H solver programming: a retarded -time algorithm 59

5.2 Flow chart of FW-H solver programming: an advanced -time algorithm with RANS . . . . . . . . . . . . . 60

5.3 Cross-section of NACA 0012 rotor . . . . . . . . . . . . . 61

5.4 Schematics of NACA 0012 rotor noise measurement set-up . . . . 62

5.5 Discretized rotor blade surface . . . . . . . . . . . . . . 63

5.6 $M_{t i p}=0.80$, comparison of predictions (blade usrface) and experiment . . . . . . . . . . . . . . . . . . 64

$5.7 M_{t i p}=0.85$, comparison of predictions (blade surface) and experiment . . . . . . . . . . . . . . . . . . . . 64

5.8 Permeable and impermeable surface cross-section . . . . . . . . 65

5.9 The integral data surfaces for NACA0012 rotor noise calculations. Permeable surfaces 1-6 has the doubled chordlength as the original blade, and spanlenggth increasing from $2 \%$ to $12 \%$. . . . . . . 66

5.10 Enlarged blade tip regions of integral surfaces . . . . . . . . . 66 
$5.11 M_{t i p}=0.80$, comparison of predictions and experiment $\ldots . .67$

$5.12 M_{t i p}=0.85$, comparison of predictions and experiment . . . . . 67

$5.13 M_{t i p}=0.88$, comparison of predictions and experiment . . . . . 68

5.14 Comparisons of experimental results at different Mach numbers, $M_{t i p}=0.88$, and $M_{t i p}=0.89 \ldots \ldots \ldots \ldots$. . . . . 68

5.15 Comparisons between the baseline SRV2 impeller and its redesigned version using inverse design method . . . . . . . . . . . . 69

5.16 Schematics of the nosie testing rig . . . . . . . . . . . 71

5.17 Numerical prediction of noise spectrum at $50000 \mathrm{rpm}$. . . . . . 71

5.18 Measured noise spectrum (see Fig. 6a in Raitor \& Neise [2008]) . 72

5.19 Aerodynamic and aeroacoustic performance comparisons between the original and redesigned compressor . . . . . . . . . . 73

5.20 Comparison of the characteristics of the original and redesign compressor ....................... 74

5.21 Mach number contour comparison between the original (LHS) and redesign $(\mathrm{RHS})$ at $15 \%$ of span . . . . . . . . . 75

5.22 Mach number contour comparison between the original (LHS) and redesign $($ RHS $)$ at $25 \%$ of span . . . . . . . . . . 76

5.23 Mach number contour comparison between the original (LHS) and redesign $(\mathrm{RHS})$ at $50 \%$ of span . . . . . . . . . . 77

5.24 Mach number contour comparison between the original (LHS) and redesign (RHS) at $75 \%$ of span . . . . . . . . . . . 78

5.25 Mach number contour comparison between the original (LHS) and redesign $($ RHS ) at $95 \%$ of span . . . . . . . . . . 78

5.26 Streamwise blade loading distributions on the main blade (lower) and splitter (upper) at $15 \%$ of span . . . . . . . . . . . 79

5.27 Streamwise blade loading distributions on the main blade (lower) and splitter (upper) at $25 \%$ of span . . . . . . . . . . 79

5.28 Streamwise blade loading distributions on the main blade (lower) and splitter (upper) at $50 \%$ of span . . . . . . . . . 80

5.29 Streamwise blade loading distributions on the main blade (lower) and splitter (upper) at $75 \%$ of span . . . . . . . . . . . 80 
5.30 Streamwise blade loading distributions on the main blade (lower) and splitter (upper) at $95 \%$ of span . . . . . . . . . . . 81

5.31 Mach number contour comparison between the original (LHS) and redesign $(\mathrm{RHS})$ at $\mathrm{LE} \ldots \ldots$. . . . . . . . . . . . . . 81

5.32 Comparison of spanwise variation of isentropic compression efficiency at the TE . . . . . . . . . . . . . . . 82

5.33 Comparison of isentropic compression efficiency in streamwise location from impeller inlet to outlet . . . . . . . . . . . . 82

6.1 Cascade notaitons . . . . . . . . . . . . . . . . . . . . . . 87

6.2 Blade loading parametrisation curve . . . . . . . . . . . 106

7.1 Overall design flow chart for the new impeller . . . . . . . . . . . 109

7.2 Meridional contour of the baseline impeller and diffuser . . . . . . 111

$7.33 \mathrm{D}$ view of the baseline impeller wheel . . . . . . . . . . . . 111

7.4 Impeller-diffuser set up in CFD . . . . . . . . . . . . . . . . 112

7.5 CFD predictions of the compressor characteristics under three meshes in Table 7.2 at speedline 1800 (about 30krpm) . . . . . . . . . . . 113

7.6 CFD set up for the compressor stage simulation . . . . . . . . . . 114

7.7 Comparisons between the predicted and measured baseline compressor's characteristics: pressure ratio . . . . . . . . . . . . . 115

7.8 Comparisons between the predicted and measured baseline compressor's characteristics: efficiency . . . . . . . . . . . . . 116

7.9 CFD prediction of Napier baseline impeller performances at $26 \mathrm{krpm} 117$

7.10 Meridional contour for the inverse designed impeller . . . . . . . . 118

7.11 Comparisons of full blade thickness at different spanwise height:

(a) Hub section; (b)25\% spanwise height; (c)50\% spanwise height;

(d)75\% spanwise height; (e)98\% spanwise height; (f)Shroud section 119

7.12 Comparisons of splitter blade thickness at different spanwise height:

(a) Hub section; (b)25\% spanwise height; (c)50\% spanwise height;

(d)75\% spanwise height; (e)98\% spanwise height; (f)Shroud section 119

7.13 Blockage factor distribution from the inlet to outlet . . . . . . . . 121

7.14 Blade loading for a 4.5 pressure ratio centrifugal impeller . . . . . 123

7.15 Blade loading for the redesigned SRV2 centrifugal impeller . . . . 125 
7.16 Optimal blade loading for the final redesign . . . . . . . . . . 127

7.17 Illustration of the definition of stacking angle in TD1 . . . . . . . 128

7.18 Simplified FEA set up. $\omega=32.5 \mathrm{krpm}$. . . . . . . . . . . . 128

7.19 Comparisons of the von Mises stress contour on the blade surface 130

7.20 Comparisons of the deformation contour on the blade surface . . . 130

7.21 Full impeller wheel with fillets used for FEA analysis . . . . . . . 131

7.22 Full impeller wheel mesh used for FEA analysis . . . . . . . . . . 132

7.23 Thermal analysis for the redesigned full impeller wheel . . . . . . 132

7.24 Stress analysis for the redesigned full impeller wheel . . . . . . . . 133

7.25 Deformation analysis for the redesigned full impeller wheel . . . . 134

7.26 Campell diagram for the redesigned full impeller wheel . . . . . . 134

7.27 Comparisons of blade loading calculated in CFX and TD1 near the

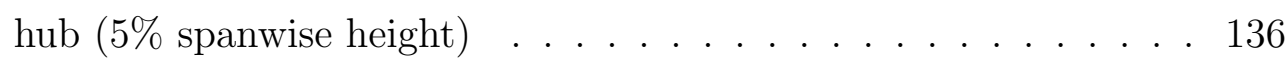

7.28 Comparisons of blade loading calculated in CFX and TD1 at the midspan (50\% spanwise height) . . . . . . . . . . . 137

7.29 Comparisons of blade loading calculated in CFX and TD1 near the blade shroud (90\% spanwise height) . . . . . . . . . . . 137

7.30 Comparisons of the blade surface pressure calculations between inviscid TD1 and viscous CFX solver . . . . . . . . . . . 138

7.31 Relative Mach number comparisons between baseline (left) and redesign (right) near the hub (10\% spanwise height) at speedline 1800, BEP . . . . . . . . . . . . . . . . . . 140

7.32 Relative Mach number comparisons between baseline (left) and redesign (right) at the midspan (50\% spanwise height) at speedline 1800, BEP . . . . . . . . . . . . . . . . . . 140

7.33 Relative Mach number comparisons between baseline (left) and redesign (right) near the shroud (90\% spanwise height) at speedline 1800, BEP . . . . . . . . . . . . . . . . . . . 141

7.34 Blade surface pressure comparisons between baseline (left) and redesign (right) near the hub (10\% spanwise height) at speedline $1800, \mathrm{BEP} \ldots \ldots \ldots \ldots 14 \ldots \ldots \ldots$ 
7.35 Blade surface pressure comparisons between baseline (left) and redesign (right) at midspan (50\% spanwise height) at speedline 1800, BEP . . . . . . . . . . . . . . . . . . . . 142

7.36 Blade surface pressure comparisons between baseline (left) and redesign (right) near the shroud (90\% spanwise height) at speedline 1800, BEP . . . . . . . . . . . . . . . . 142

7.37 Relative Mach number comparisons between baseline (left) and redesign (right) near the impeller exit at speedline 1800, BEP . . 143

7.38 Relative positions between the impeller and microphones for numerical calculations . . . . . . . . . . . . . . . . . . . . . . . . 144

7.39 Comparisons of the acoustic pressure signature in one period between the baseline and redesigned impeller near the choke: single blade . . . . . . . . . . . . . . . . . . . . . 145

7.40 Comparisons of the total acoustic pressure signature in one period between the baseline and redesigned impeller wheels near the choke: all blades . . . . . . . . . . . . . . . . . . 146

7.41 Comparisons of sound pressure level in the frequency domain between baseline and redesign near choke . . . . . . . . . . . . . . 146

7.42 Comparisons of the acoustic pressure signature in one period between the baseline and redesigned impeller near the BEP: single blade . . . . . . . . . . . . . . . . . . . . . . . . 147

7.43 Comparisons of the total acoustic pressure signature in one period between the baseline and redesigned impeller wheels near the BEP:

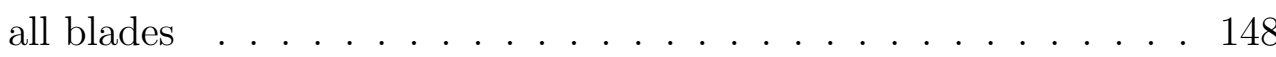

7.44 Comparisons of sound pressure level in the frequency domain between baseline and redesign near the BEP . . . . . . . . . . . . . 148

7.45 Comparisons of the predicted pressure ratio between baseline and redesign at multiple speedlines . . . . . . . . . . . . . . . . . . . . 149

7.46 Comparisons of the predicted isentropic efficiency between baseline and redesign at multiple speedlines . . . . . . . . . . . 150

8.1 Schematic of turbocharger control facility . . . . . . . . . . . . . 153

8.2 Schematic of oil control facility . . . . . . . . . . . . . . . . . 154 
8.3 Comparisons between the predicted and measured redesigned compressor's characteristics: pressure ratio . . . . . . . . . . . 155

8.4 Comparisons between the predicted and measured redesigned compressor's characteristics: efficiency . . . . . . . . . . . . . . 156

8.5 Comparisons of the measured stage pressure ratios of baseline and redesign compressors at different operating speedlines . . . . . . 157

8.6 Comparisons of the measured stage efficiency (non-dimensionalised) of baseline and redesign compressors at different operating speedlines 158

8.6 (continued) Comparisons of the measured stage efficiency (nondimensionalised) of baseline and redesign compressors at different operating speedlines . . . . . . . . . . . . . . . . . . . . 159

8.7 Comparisons of the two compressors' performance and their operating ranges. (Experimental measurements) . . . . . . . . . . 160

8.8 Different noise measurement positions. (MP2 and 3 are symmetric to MP4 and 5) . . . . . . . . . . . . . . . . . 161

8.9 Sound level measured at the inlet for different positions . . . . . . 162

8.10 Average sound levels measured at position 2, 4 and position 3, 5 . 163

8.11 Comparisons of the measured spectral characteristics of baseline and redesign impellers at speedline 1300 . . . . . . . . . . . 163

8.12 Comparisons of the measured spectral characteristics of baseline and redesign impellers at speedline 1400 . . . . . . . . . . . 164

8.13 Comparisons of the measured spectral characteristics of baseline and redesign impellers at speedline 1500 . . . . . . . . . . 165

8.14 Comparisons of the measured spectral characteristics of baseline and redesign impellers at speedline $1600 \ldots \ldots 6$

8.15 Comparisons of the measured spectral characteristics of baseline and redesign impellers at speedline $1700 \ldots$. . . . . . . . 167

8.16 Comparisons of the measured spectral characteristics of baseline and redesign impellers at speedline $1800 \ldots \ldots$. . . . . . . . . 168

8.17 Comparisons of the measured spectral characteristics of baseline and redesign impellers at speedline 1900 . . . . . . . . . . . 169 
8.18 Comparisons of the relative Mach number distribution between the baseline (left) and redesign (right) impeller near the hub at speedline $1000 \ldots \ldots \ldots$. . . . . . . . . . . . 171

8.19 Comparisons of the relative Mach number distribution between the baseline (left) and redesign (right) impeller at the midspan at speedline $1000 \ldots \ldots \ldots$. . . . . . . . . . . . 172

8.20 Comparisons of the relative Mach number distribution between the baseline (left) and redesign (right) impeller near the shroud at speedline 1000 . . . . . . . . . . . . . . . . . 173

8.21 Comparisons of the relative Mach number distribution between the baseline (left) and redesign (right) impeller near the hub at speedline 1500 . . . . . . . . . . . . . . . . . . . . . . 174

8.22 Comparisons of the relative Mach number distribution between the baseline (left) and redesign (right) impeller at the midspan at speedline 1500 . . . . . . . . . . . . . . . . 175

8.23 Comparisons of the relative Mach number distribution between the baseline (left) and redesign (right) impeller near the shroud at speedline 1500 . . . . . . . . . . . . . . . . . . 176

8.24 Comparisons of the relative Mach number distribution between the baseline (left) and redesign (right) impeller near the hub at speedline 1800 . . . . . . . . . . . . . . . . . . . . . 177

8.25 Comparisons of the relative Mach number distribution between the baseline (left) and redesign (right) impeller at the midspan at speedline $1800 \ldots \ldots$. . . . . . . . . . . . . 178

8.26 Comparisons of the relative Mach number distribution between the baseline (left) and redesign (right) impeller near the shroud at speedline 1800 . . . . . . . . . . . . . . . . . . . . 179

8.27 The conventional throat area schematic . . . . . . . . . . . . 179

8.28 Comparisons of the relative Mach number distribution between the baseline (left) and redesign (right) near the diffuser hub at speedline $1800 \ldots \ldots$. . . . . . . . . . . . . . . 180 
8.29 Comparisons of the relative Mach number distribution between the baseline (left) and redesign (right) at the diffuser midspan at speedline 1800 . . . . . . . . . . . . . . . . . . . 181

8.30 Comparisons of the relative Mach number distribution between the baseline (left) and redesign (right) near the shroud at speedline 1800182

8.31 Tip leakage flow behaviours for the baseline (left) and redesign (right) blade channels at the peak efficiency point . . . . . . . . . 182

8.32 Surface streamline structures on the baseline and redesign impeller at the peak efficiency point . . . . . . . . . . . . . . . 183

8.33 Mach number distributions on the suction surface of baseline and redesign impeller at the peak efficiency point . . . . . . . . . . . . 184

8.34 Comparisons of velocity vectors on the full blade suction surface between the baseline (left) and redesign (right) . . . . . . . . . . . 184

8.35 Comparisons of velocity vectors on the splitter blade suction surface between the baseline (left) and redesign (right) . . . . . . . . 185

8.36 Comparisons of the exit flow Mach number at $1 \mathrm{~mm}$ downstream of trailing edge for the baseline (left) and redesign (right) impeller at the peak efficiency point . . . . . . . . . . . . . . . . 185

8.37 Comparisons of the relative Mach number near the impeller LE between the baseline (left) and redesign (right) impeller at the peak efficiency point . . . . . . . . . . . . . . 186

8.38 Comparisons of the relative Mach number near the hub between the baseline (left) and redesign (right) impeller at the peak efficiency point . . . . . . . . . . . . . . 186

8.39 Comparisons of the relative Mach number at the midspan between the baseline (left) and redesign (right) impeller at the peak efficiency point . . . . . . . . . . . . . . . 187

8.40 Comparisons of the relative Mach number near the shroud between the baseline (left) and redesign (right) impeller at the peak efficiency point . . . . . . . . . . . . . . . . . . 188

8.41 Comparisons of the blade surface pressure near the hub between the baseline and redesign at the peak efficiency point . . . . . . . 188 
8.42 Comparisons of the blade surface pressure at the midspan between the baseline and redesign at the peak efficiency point . . . . . . . 189

8.43 Comparisons of the blade surface pressure near the shroud between the baseline and redesign at the peak efficiency point . . . . . . . 190

8.44 Comparisons of the pressure recovery of the same vaned diffuser when matched with the baseline and redesigned impellers . . . . . 190

8.45 Comparisons of the Mach number at the diffuser inlet between the baseline (left) and redesign (right) impeller . . . . . . . . . . . . 191

8.46 Comparisons of 3D streamlines in the same diffuser channel with incoming flow from baseline (left) and redesign (right) impeller exit 191

8.47 Comparisons of surface pressure on the midspan position of the diffuser with baseline and redesign incoming flow conditions . . . 192 


\section{List of Tables}

2.1 Dominant mechanisms for subsonic noise generation . . . . . . . 20

2.2 Dominant mechanisms for supersonic noise generation . . . . . . . 20

5.1 Regimes of integral formulations for subsonic or transonic cases . 63

5.2 SRV2 compressor parameters . . . . . . . . . . . 70

7.1 Baseline impeller parameters . . . . . . . . . . . . . . . . 110

7.2 Three different meshes used for the mesh-independence study . . . 112

7.3 Spanwise $r V_{\theta}^{*}$ distribution . . . . . . . . . . . . . . . 122

7.4 Aluminium alloy 2618A mechanical properties: no thermal effects considered . . . . . . . . . . . . . . . . . . . . . 129

7.5 Normalised results from the simplified FEA modelling . . . . . . . 131

7.6 Material constants of Aluminium alloy 2618A . . . . . . . . . 133 


\section{Nomenclature}

\section{Roman Symbols}

$\boldsymbol{l} \quad$ streamwise length vector in the blade surface

$\boldsymbol{u} \quad$ fluid velocity

$\boldsymbol{V} \quad$ velocity vector in the stationary frame

$\boldsymbol{v} \quad$ blade velocity

$\boldsymbol{W} \quad$ velocity vector in the relative frame

$\boldsymbol{x} \quad$ observer position

$\boldsymbol{y} \quad$ source position

M Mach number vector

n normal vector to integral surface

$\mathbf{r} \quad$ radiative vector

$B \quad$ number of blades

$B_{f} \quad$ stream thickness factor

c speed of sound

$c_{p} \quad$ specific heat capacity

$f \quad$ closed source surface 
G Green function

$g \quad$ collapsing sphere

$H \quad$ Heaviside function (in Chapter 3 only)

$H \quad$ stagnation enthalpy

$h \quad$ specific enthaply

$l_{c} \quad$ chord length

$M_{0} \quad U_{0} / c$, convective flow Mach number

$M_{r} \quad$ Mach number in radiative direction

$p \quad$ static pressure on the blade surface

$p_{L}^{\prime} \quad$ loading noise

$p_{T}^{\prime} \quad$ thickness noise

Q volume flow

$r \quad$ source-observer distance

$r \overline{V_{\theta}} \quad$ swirl velocity/circulation/specific Euler work

$S \quad$ generic integral data surface

$s \quad$ entropy

$S^{\prime} \quad$ sawtooth function

$S_{b} \quad$ blade surface

$S_{p} \quad$ porous surface

$t \quad$ observer/reception time

$T_{i j} \quad$ Lighthill stress tensor

$U_{0} \quad$ medium velocity 
$U \quad$ inernal energy

\section{Greek Symbols}

$\alpha \quad$ vorticity sheet

$\beta \quad \sqrt{1-\left(\frac{U_{0}}{c}\right)^{2}}$

$\delta_{i j} \quad$ Kronecker tensor

$\delta() \quad$ delta function

$\eta \quad$ isentropic efficiency

$\kappa \quad$ splitter work ratio

$\lambda \quad$ Clebsch scalar

$\mu \quad$ Clebsch scalar

$\omega \quad$ angular velocity

$\phi \quad$ potential function

$\pi \quad \simeq 3.1415926 \ldots$

$\psi \quad$ stream function

$\rho \quad$ fluid density

$\rho_{0} \quad$ ambient density

$\sigma_{i j} \quad$ viscous tensor stress

$\tau \quad$ emission/source time

$\vartheta \quad$ angle between $\hat{n}$ and $\hat{r}$

$\Omega \quad$ vorticity vector

$\Gamma \quad$ bound circulation (in Chapter 6 only)

$\Gamma \quad$ intersection curve 
$\Pi \quad$ pressure ratio

$\Sigma \quad$ emission surface

\section{Superscripts}

* meridional normalisation

** conjugate

$+\quad$ upper surface

- $\quad$ lower surface

$\overline{()} \quad$ pitchwise/tangential/circumferential average

1 superscript index

$\widetilde{()} \quad$ periodic component

j superscript index

\section{Subscripts}

$0 \quad$ subscript index

am artificially modified

bl blade surface

$h \quad$ hub

mbl meridional component on the blade surface

$s \quad$ shroud

\section{Other Symbols}

$\square^{2} \quad$ convective wave operator

$\square^{2} \quad$ wave operator

\section{Acronyms}


BEP best efficiency point

BPF blade passing frequency

IGV inlet guide vane

LE leading edge

MP measurement point

OGV outlet guide vane

RSM response surface method

TCN tip clearance noise

TE trailing edge 
"A thousand miles begin with a single step."

Lao-Tze (500 - 600 BC)

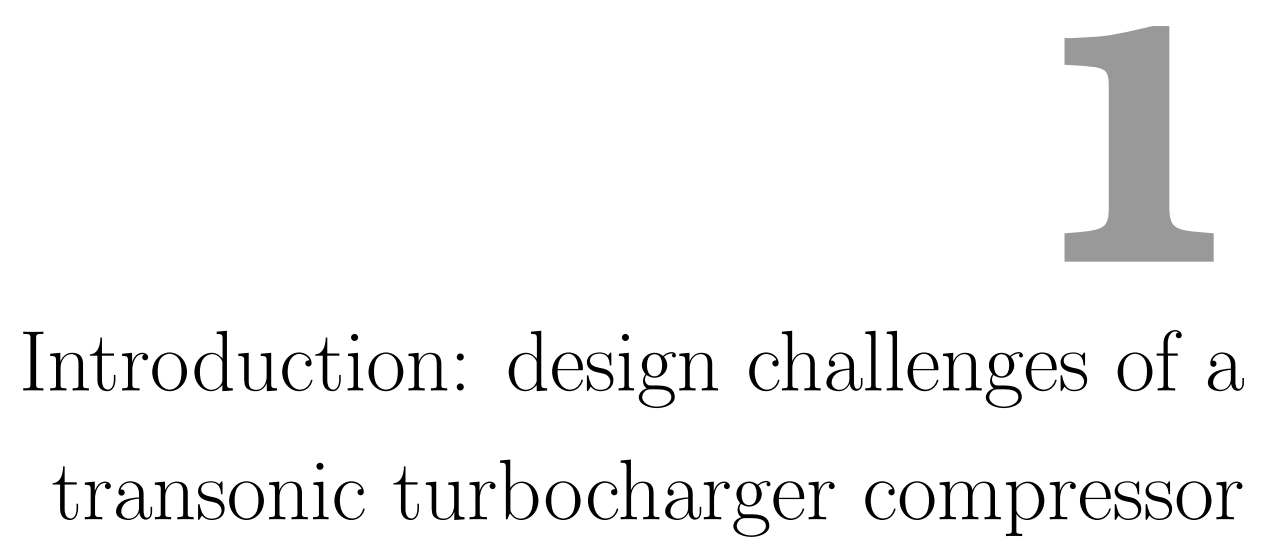

\subsection{Research background}

Turbochargers play a very important role in the modern turbomachinery industry, which are commonly used for transportations such as automotives, trucks, ships, and helicopters. A turbocharger consists of two key components, centrifugal compressor and radial/axial flow turbine. The compressor is connected to the turbine through a coaxial shaft. Turbine blades can be driven to spin by exhausted gases from the engine, and the torque is transmitted through the shaft to rotate the compressor into which air is then extracted. In the fast spinning blade channels, the pressure and kinetic energy of air is increased significantly. After leaving the impeller passages, air flows into a diffuser where the deceleration occurs and high pressure air is obtained. A scroll collector or volute is placed after the diffuser to further decelerate the flow and orient it into the right trajectory 


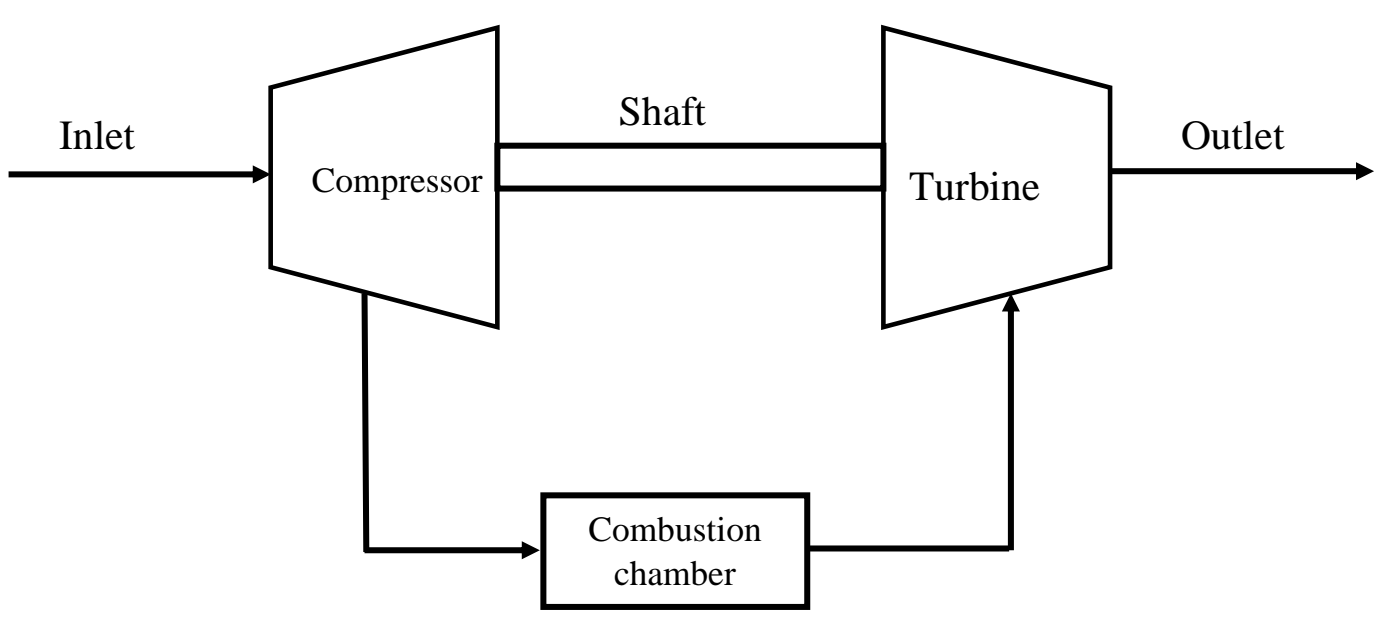

Figure 1.1: Schematic of a turbocharger cycle

before going to an engine for burning. Figure 1.1 shows a brief schematic of the entire turbocharging process.

In this work only compressor will be studied. The usual way to characterise a turbocharger compressor's performances is to use the compressor map, which includes the mass flow rate, pressure ratio, efficiency and turbo speeds. Such a map defines the compressor's operating range, capabilities of boosting pressure and aerodynamic efficiency. In addition to that, the modern industry has an extra factor to evaluate a compressor's performances, which is the aerodynamically generated sound. In the U.S and many countries in the Europe the requirement for low noise working environment is getting ever stricter. It poses a very big challenge for the turbomachinery community given that designing transonic compressors with only high aerodynamic performances is already quite difficult. The purpose of this project is to explore the design possibilities of a transonic centrifugal compressor for a turbocharger to get both aerodynamic and aeroacoustic performances enhanced.

In heavy duty applications the compressor is demanded to deliver very high pressure ratio (typically 3.5: 1 for automobiles and over 5:1 for large marine ships) so as to increase the combustion efficiency. Of these scenarios the flows in the compressor are always transonic and have very complex structures. The strongly distorted flow behaviours can induce a great deal of losses to the compres- 


\section{INTRODUCTION: DESIGN CHALLENGES OF A TRANSONIC TURBOCHARGER COMPRESSOR}

sor efficiency and generate a large amount of unfavourable aerodynamic sound. This type of aerodynamic noise lies in the field of aeroacoustics, which is an interdisciplinary combining aerodynamics and theory of acoustics. It mainly focuses on investigating the sound generated by turbulent flows and aerodynamic forces interacting with solid surfaces. Although much attention has been paid to studying this phenomenon in the past few decades, the reduction of turbomachinery noise still remains very challenging due to complexities of the acoustic and flow field, particularly for transonic turbomachines. More details about the challenges of modern turbomachinery aeroacoustics research can be found in a review paper by Peake \& Parry [2012].

Acoustic liner is a common passive way to reduce the compressor's noise, but it harms the aerodynamic efficiency. An active way would be more favoured since it can help to reduce the noise at the source, i.e., a successful design for compressor itself that can retain good aerodynamic performances and limit the noise levels without using other supplementary tools.

The conventional way of designing a compressor impeller is to parametrise the blade profile using three-dimensional CAD modelling. Once the blade geometry is fixed, the aerodynamic and aeroacoustic performances can be predicted using computational simulations. The blade profiling must be repeated until the target performances of a compressor are met. The blade parametrisations can be coupled with advanced optimization algorithms and DoE (design of experiment) method to seek an optimal design. This methodology has been proven to be effective for many industrial applications. However, there are still some intrinsic disadvantages embodied in such method listed below:

1. The design period is generally very long.

2. It is often DP-locked (design point locked) rather than multi-points design.

3. The CFD simulations require an enormous amount of computational resources and do not provide guide lines for the refinement of blade profiling in the followed design process.

4. Updating the blade profiles is time-consuming. 


\section{INTRODUCTION: DESIGN CHALLENGES OF A TRANSONIC TURBOCHARGER COMPRESSOR}

5. The space for exploring the optimal design is constrained.

The current trend of seeking down-sizing and quieter designs has pushed the traditional method to its bottleneck.

An alternative way for the blade design is the three dimensional inverse design method. The conventional design method is nevertheless quite intuitive at first glance, which is a process starting from CAD drawing of an impeller wheel to flow simulations, therefore it is also called the direct design method. The inverse design, as its name suggests, fundamentally differs from the direct method at the starting point. First, the flow field has be to prescribed. Following that, the blade profile is automatically computed in an iterative way until the final blade profile can give the specified flow field, hence, an inverse process. The optimisation for an impeller blade coming out of inverse design is then naturally related to the prescription of the flow field rather than the blade profiling. It is not difficult to see that the advantage of the inverse design is its possibility to take advantage of the knowledge and information from CFD calculations of the flow field. The aerodynamic behaviours of the flow on the blade surface largely determine the performance of a compressor, therefore, one can specify a modified flow field that is devised to lead to better performances of a compressor than the previous step's design. The aeroacoustic theory has also suggested the close relations between the flow field on the blade surface and noise generations. Thus, it is possible to use the inverse design method to design compressor impellers with multi-objectives.

\section{$1.2 \quad$ Research objectives}

This project is motived to redesign a transonic centrifugal impeller used for marine applications by Napier Turbocharger, Ltd. The selected final design will be prototyped and tested. Its macro objectives are three-fold:

- to redesign a transonic centrifugal impeller that can improve both aerodynamic and aeroacoustic performances when compared with the baseline design;

- to expound the design method for redesigning and optimising impellers 


\section{INTRODUCTION: DESIGN CHALLENGES OF A TRANSONIC TURBOCHARGER COMPRESSOR}

so further design work with multi-objective and multi-disciplinary can be carried out in the frame work of 3D inverse design;

- to shed lights upon the future multi-point, multi-objective, and multidisciplinary design of transonic centrifugal compressors.

The micro objectives include:

- to explore the numerical prediction method for rotating rotors in both subsonic and transonic regimes;

- to systemically establish a model that can be used for rotor induced noise evaluations;

- to use the inverse design method to design a new impeller that has improved performances over the baseline one, and particularly, the highest efficiency zone needs to be shifted to the higher turbo speeds on the compressor map;

- to investigate the impeller's performance using CFD and the developed aeroacoustic model;

- to validate the predicted performances of the new impeller experimentally and compare with the baseline back to back;

- to analyse the flow field differences between baseline and redesigned impeller using the detailed CFD calculations.

\subsection{Thesis structure}

The rest of the thesis will be structured in the following way:

- Chapter 2 presents a literature survey mainly covering two topics, the aerodynamic design and aeroacoustics of turbomachines. These two topics both have had a long development history and a very broad range, and it is only intended to review those works that can relate these two disciplines focusing on a historical and application point of view. 


\section{INTRODUCTION: DESIGN CHALLENGES OF A TRANSONIC TURBOCHARGER COMPRESSOR}

- Chapter 3 is devoted to the theoretical formulations of the aeroacoustics that can be used for turbomachine noise predictions. The acoustic genealogy dates back to the well-known Lighthill acoustic analogy and FW-H equation with the convective term.

- Chapter 4 develops a solver that is an extension to the well-known Farassat Formulation 1A for the rotor induced noise in the steady-state form. An algorithm has also been developed for the numerical implementation of this solver. A program named as NoiseComp has been written in FORTRAN 90 to perform acoustic calculations, and its source code and user manual were given to Napier Turbochargers.

- Chapter 5 shows two applications of the acoustic solver, including a 2D helicopter rotor's noise calculations at various tip Mach numbers, and the 3D SRV2 centrifugal impeller at transonic conditions. The aeroacoustic predictions are compared with the published experimental data for validations.

- Chapter 6 explains the theory used in the inverse design code TURBOdesign1. Most of the key equations have been re-derived and presented in a systematic way in this chapter.

- Chapter 7 details the design procedures for the new impeller. The aerodynamic and aeroacoustic performances of Napier's baseline wheels are analysed using CFX simulations and acoustic solver, which are validated with test data.

- Chapter 8 discusses the test results of the redesigned impeller and compares its performances with the baseline impeller. Detailed flow analyses of the two designs from CFD solutions are also discussed.

- Chapter 9 gives some conclusive remarks of the current thesis and suggestions for future research and studies. 
"If I have seen further than others, it is by standing upon the shoulders of giants."

Sir Isaac Newton (1643 - 1727)

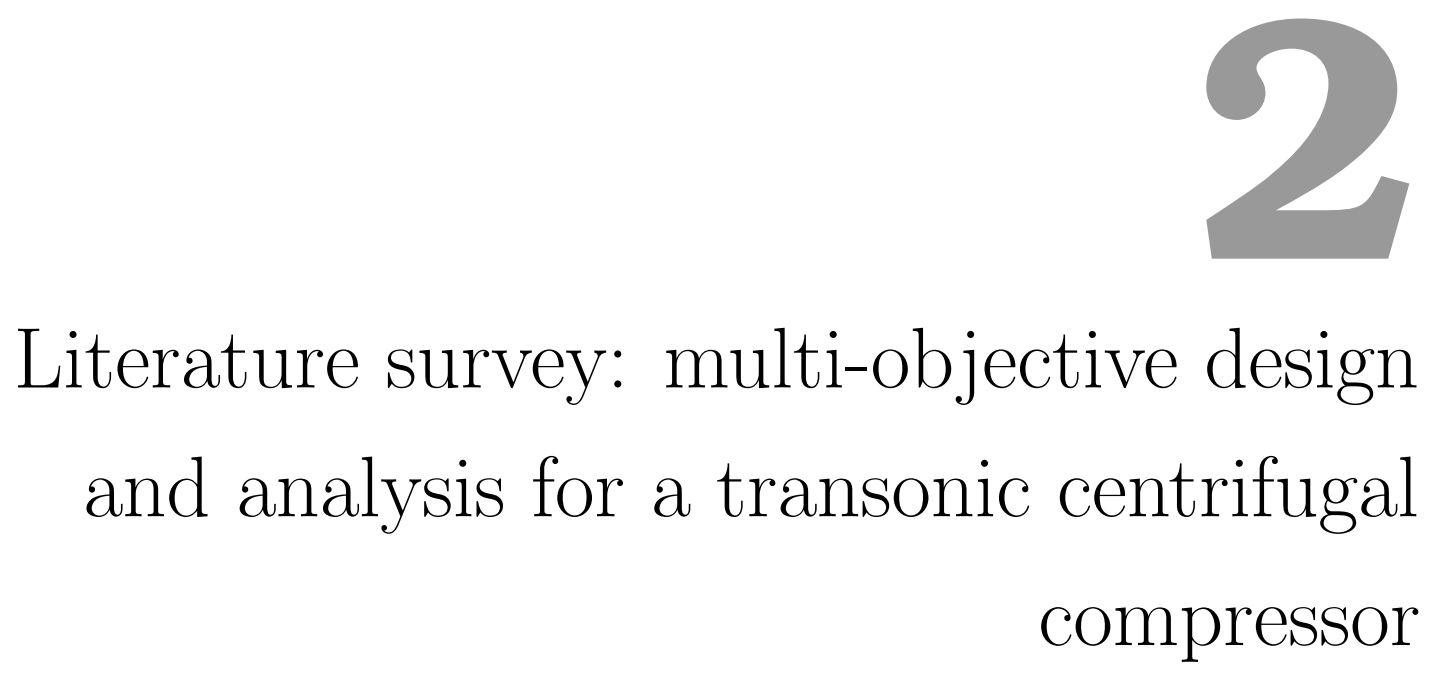

In the last chapter, the background and objectives of this project have been clearly presented and defined. This chapter is dedicated to reviewing the work that has been done for designing turbomachinery components with multi-objectives. From the literature survey, one can easily find an enormous amount of research articles studying all different turbomachinery components using computational and experimental techniques. It is, however, not intended (neither realistic) to critically review all the existing design and analysis methodologies. Since the purpose of this project is to redesign a transonic compressor with lower noise and better efficiency at higher speeds compared to the baseline design, our efforts for the literature review will be concentrated on these three fields:

1. features of the noise spectrum of turbocharger compressors; 


\section{LITERATURE SURVEY: MULTI-OBJECTIVE DESIGN AND ANALYSIS FOR A TRANSONIC CENTRIFUGAL COMPRESSOR}

2. aeroacoustic noise prediction approach for turbomachines;

3. inverse design method for turbomachines.

The novelty of the current work will naturally emerge in the contextualisation of previous works on these three topics.

\subsection{Compressor stage components}

The term "compressor stage" normally refers to a system including a few different components which will be mentioned many times in this thesis. In this section we intend to give a brief overview and definition of these components for the convenience that we would not need to explain them in different contexts again.

Dixon \& Hall [2010] classified a turbomachine as any device through which the energy can be transferred either from or to the fluid by the rotating blade rows. One of the two main categories of turbomachines absorbs energy to increase the fluid pressure or head, such as, compressors, pumps, and fans; the other one converts the potential and kinetic energy of a flow into the mechanical energy, such as hydraulic, gas, and steam turbines. Depending on the direction of the flow passing through the rotor channels, the turbomachines can be further subcategorised into radial and axial flow turbomachines. The flow path of a radial turbomachine is completely or mainly in a plane that is perpendicular to the rotation axis whereas the flow path of an axial type is completely or mainly in a plane that is parallel to the rotation axis.

A centrifugal/radial compressor is usually constituted of the following ingredients and also schematically illustrated in Fig. 2.1.

1. Meridional contour

The meridional contour, shown in Fig. 2.1, is perhaps the first important geometric concept for the analysis of any turbomachinery components. It presents a channel formed by the hub and shroud, both of which are solid disks confining the flow. The hub and shroud are projected into the $R-z$ plane that is often used in a cylindrical coordinates. $R$ and $z$ refer to the radial and axial direction respectively, and $z$ axis is usually taken as the rotation axis. 


\section{LITERATURE SURVEY: MULTI-OBJECTIVE DESIGN AND ANALYSIS FOR A TRANSONIC CENTRIFUGAL COMPRESSOR}

\section{Impeller}

Impeller is the most important part of a compressor. The design or optimisation of a compressor usually means modifying the impeller geometry to get the target performances. For a turbocharger compressor, the impeller normally has a few pairs of full/long and splitter/short blades. The standard industrial compressor often has only the full blades. The frontal part of the blades are usually known as inducer, which is very significant for determining the compressor performances, particularly in determining the efficiency and operating range. This is the area where the most complicated flow behaviours occur, such as shocks, flow separations, reverse flow and vortex sheddings. The exit of the blade is usually known as the exducer, which can have a cut-off or rounded edge. It may or may not be leaned either in the way of its tip leading the root or its root leading the tip. The lean angle of the exducer is quite critical to determine the mechanical stress of the impeller wheel and also the jet-wake flows interacting with the next component, diffuser.

\section{Diffuser}

Diffuser is the place where exit flow from blade passages decelerates. There are in general two types of diffusers, namely, vaneless and vaned diffuser. The vaneless diffuser is nothing more than an empty section formed by the hub and shroud disk. If one puts some vanes in this space, it then becomes a vaned diffuser. The vaned diffuser can be used to enhance the pressure ratio and efficiency but it will considerably reduce the compressor operating range when compared with the vaneless diffuser. A comprising solution is to use the so called low solidity vaned diffuser(LSVD), which has fewer vanes than the conventional vaned diffuser. The LSVD normally causes smaller losses of choke and stall margins when compared with a vaned diffuser.

4. Volute

A volute, sometimes also known as the scroll collector, is commonly used at the vaneless/vaned diffuser downstream. The flows are diffused and decelerated inside the volute before going into a discharge pipe, which further converts the kinetic energy into the static pressure. 


\section{LITERATURE SURVEY: MULTI-OBJECTIVE DESIGN AND ANALYSIS FOR A TRANSONIC CENTRIFUGAL COMPRESSOR}

\section{Ported shroud}

The ported shroud is a casing treatment device placed on the shroud of the impeller wheel, and it is commonly put at an axial position in the vicinity of the splitter leading edge. This device is well-known for its capability to improve the stall margin for a compressor. However, its working mechanism of postponing the compressor instability is still not too well understood yet. In recent years a few articles worth particular attention have been published by some turbomachinery specialists from Honeywell Turbo Technologies, such as Chen \& Lei [2013]; Gancedo et al. [2014]. In short, they concluded that the enhanced compressor stability due to the ported shroud is a result of eliminating swirling back flow at the inducer and recirculating it into the impeller channel. This device is of high significance for improving the compressor operating range. The turbomachinery experts from HTT (Honeywell Turbo Technologies) and CTT (Cummins Turbo Technologies) have conducted optimisations for its design, as can be found in the works by Yin et al. [2009] and Sivagnanasundaram et al. [2012].

All these components have both aerodynamic and aeroacoustic effects on the compressor performances. These five components comprise a system in a highly nonlinear manner, and their effects on the flow field interact with each other. A thorough optimisation for every single component with multi-objectives is not realistic. In the current work, only the impeller will be studied.

\subsection{Classifications of the turbocharger noise}

The noise sources of a turbocharger are quite diversified. The main sources can be of either aerodynamic or mechanical nature. In the book by Hung [2013] the turbocharger noise sources are classified as nine different types enumerated below:

1. Pulsation whistle

It is caused by the slight differences in the compressor wheel chamber volumes due to the variations of manufacturing processes.

2. Rotational noise

This is a rotating-blade-related noise by compressor or turbine blade rows. 
$\mathbf{R}$

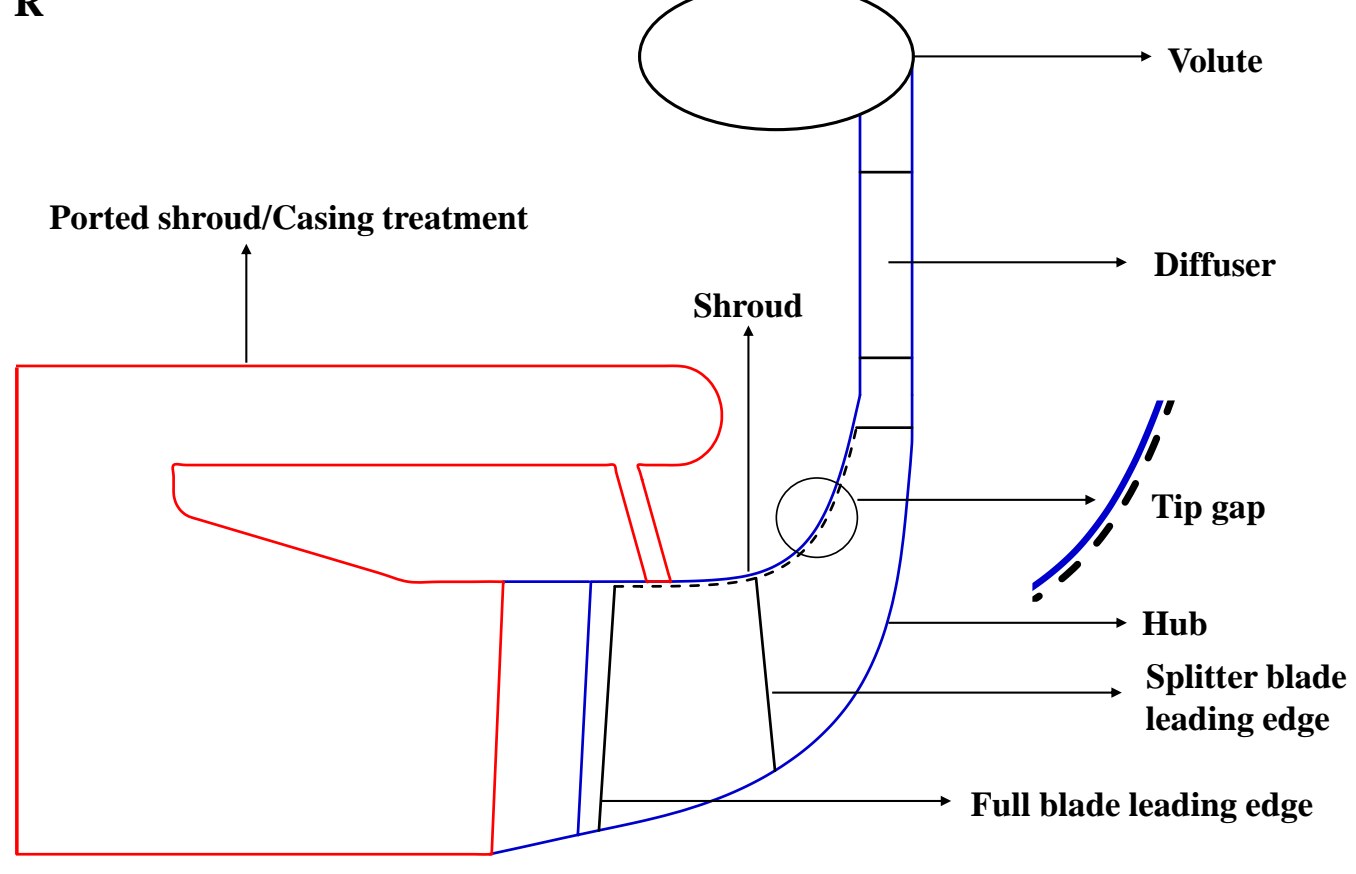

Figure 2.1: Meridional view of a centrifugal compressor stage

It has very high frequencies between $8-18 \mathrm{kHz}$ or even higher, which results from the number of blades and the turbo shaft speed.

3. Growling noise

This noise is normally observed when the compressor starts to stall, which results in a partial flow separation at the suction side near the impeller outlet. Its spectrum has a wide band between 1200 and $3500 \mathrm{~Hz}$.

4. Whining noise

When the whining noise occurs this is an indication of a deep surge condition. The air recirculates from the compressor outlet to the inlet. Its spectrum ranges from 800 to $2700 \mathrm{~Hz}$ and this noise propagates to the compressor inlet.

5. Unbalance whistle

The unbalance whistle noise is due to the unbalanced rotor and unbalanced change of rotor after a long operating time. 


\section{LITERATURE SURVEY: MULTI-OBJECTIVE DESIGN AND ANALYSIS FOR A TRANSONIC CENTRIFUGAL COMPRESSOR}

6. Constant tone (howling)

This type of noise is triggered by oil whirling in the oil film bearing.

7. High-order harmonic noise

This noise type is caused by an inappropriate design of the oil damper clearance between the bearing outer race or cartridge and bearing house.

8. Wear noise

It occurs in turbochargers using rolling element bearings if wear defects of balls, rollers, cage, inner and out races take place.

9. Crackling noise

It mostly takes place in wastegated turbochargers in which the waste gate vibrates about its pivot axis.

The first four noise types are due to flow aerodynamics in the compressor and turbine wheels. The others are related to the solid structure, which is not of interest for this project.

The rotational, growling and whining noise types are closely related to impeller blades and the compressor's aerodynamic characteristics. The rotational noise is generated by either the compressor or turbine wheel and it usually occurs at high frequencies. The growling noise can be heard when there is partial reversal of the air in the compressor, indicating the stalling starts to happen. The whining noise is due to the complete recirculation of air inside the compressor from outlet to inlet, which means the compressor is in the deep surge mode. The choke and stall related noise is undoubtedly very important for a compressor. However, the numerical evaluations of such noises are quite difficult without a great deal of computational resources, especially for stall and surge noise. The evaluations would have to employ the most advanced transient simulations to model. Additionally, the experimental validations are quite difficult without the most advanced acoustic chamber and testing facilities. It is not our intention to devise a thorough computational strategy to accurately simulate the compressor's entire noise spectrum. Our primary concern is to get a viable design solution to reduce the compressor noise emissions while maintaining good aerodynamic performances. 


\section{LITERATURE SURVEY: MULTI-OBJECTIVE DESIGN AND ANALYSIS FOR A TRANSONIC CENTRIFUGAL COMPRESSOR}

The next section will review the rotor related acoustic theories and their expansions to the modern prediction method for the aerodynamic noise.

\subsection{Theory of aerodynamic sound: aeroacous- tics}

In the last section a detailed classification of turbocharger noise is presented from an engineering perspective. As has been understood, the sound perceived from a turbocharger is multi-directional, multi-dimensional, and multi-sourced. In reality these different types of noise always happen simultaneously, and it is not possible to disentangle and handle them individually. Viewing this problem from an aerodynamic design point of perspective, it is reducing the rotor induced noise worth the most effort. Therefore, this section is dedicated to reviewing:

1. the previous work on the mechanism and prediction methods for rotorinduced noise;

2. mechanisms of noise generation in turbomachines and the features of sound spectrum.

\subsubsection{Pre-acoustic analogy era: rotor noise physics}

In the 1930s, researchers identified that the thickness and loading noise are the two major sources for a propeller noise field, of which the former is due to the displacement of flow by the propeller and the latter by the accelerating force on the fluid by the moving blade. Demming [1938] and Ernsthausen [1937] contributed to the theory of thickness noise and Gutin [1948] studied the loading noise. In the early 1950s, Garrick \& Watkins [1954] extended Gutin's work by replacing the normal pressure distribution over a propeller by a distribution of acoustic pressure doublet acting on blade subject to uniform rectilinear motion for a rotating propeller in forward flight. These works formed the fundamental methodology for predicting the rotor noise before Lighthil's acoustic analogy theory is developed. 


\section{LITERATURE SURVEY: MULTI-OBJECTIVE DESIGN AND ANALYSIS FOR A TRANSONIC CENTRIFUGAL COMPRESSOR}

\subsubsection{Acoustic analogy era: Lighthill and FW-H}

In 1952, Lighthill developed his well-known acoustic analogy equation by rearranging the momentum and continuity equation. The reason why it is called an acoustic analogy is because this equation is not based on the physics of flowinduced noise but rather a way of representing the sound generation by the governing equations of a compressible flow. This acoustic analogy equation has a wave operator in the LHS (left hand side) and a source term in the RHS (right hand side), thus making it an inhomogeneous wave equation. Initially, this theory was to study the jet flow noise, and no solid boundary effects were considered. Nevertheless, it is widely regarded that Lighthill's work gave birth to modern aeroacoustics. Curle [1955] extended Lighthill's theory and studied the solid boundary effects upon the aerodynamic sound, but Curle's work only considered the stationary solid boundaries which are quite rare in industrial applications. In the late 1960s, Ffowcs-Williams \& Hawkings [1969] started from Lighthill's theory and further extended his work by exploiting the powerful generalised function theory. They included the moving solid boundary effects and developed the socalled FW-H equation which has been the foundation for the aeroacoustic studies ever since. The FW-H equation is a general form of Lighthill's acoustic analogy equation and characterizes the noise field consisting of three parts, monopole, dipole, quadrupole, of which the former two are surface terms and the final one is a volume term. Since the mid of 1970s, Farassat can perhaps be regarded as the most active researcher of aeroacoustics. Based on the FW-H equation, Farassat [1975] developed two formulations known as Farassat Formulation 1 and Formulation $1 \mathrm{~A}$, of which the latter is easier to implement and more accurate than the former. It has been used most for rotor noise predictions and has shown good robustness and reliability. This formulation is a surface integral equation using the retarded time algorithm, which means the observer time series are given first and then the corresponding time when the sound signals are emitted are computed accordingly. Farassat Formulation 1A has a very good reputation in evaluating the noise generated by blades rotating subsonically. Many previous studies have shown that Formulation 1A can give accurate prediction for a rotor with a tip Mach number less than 0.80. It has been adopted over past two 


\section{LITERATURE SURVEY: MULTI-OBJECTIVE DESIGN AND ANALYSIS FOR A TRANSONIC CENTRIFUGAL COMPRESSOR}

decades for helicopter rotors, and propeller noise predictions. However, the limit of this formulation is that it fails to predict the noise accurately enough in the presence of transonic cases. This is because the original Farassat Formulation 1A neglects the quadrupole term, and this highly nonlinear volume term can have significant contribution to the total noise field so that it should not be neglected under transonic/supersonic circumstances.

\subsubsection{Recent research on the aerodynamic noise predic- tion approach: an integration of FW-H and CFD}

Farassat's Formulations 1 and $1 \mathrm{~A}$ are the most significant outcome of the aeroacoustic research from the 1970s to 1990s. These two formulations can lead to adequately good results without resorting to a very large amount of computational resources, especially when dealing with low Mach number aerodynamic noise. Since the 1990s, much work has been dedicated to finding feasible methods for accurate predictions of supersonic aerodynamic noise, to which the quadrupole source is mainly attributed. This noise source was not included in Farassat's Formulations. Brentner [1994]; Farassat [1986]; Farassat \& Brentner [1998] and Ianniello [1999, 2007] made much effort to directly evaluate the quadruole source term, but the methods they discovered are all difficult to implement, particularly in the construction of the acoustic surface for the quadrupole source.

The fast-developing CFD (Computational Fluid Dynamics) technology in 1990s shed light upon this difficult problem. di Francescantonio [1997] suggested another perspective to analyse the rotor-induced noise. The idea was that the integral surface used in Farassat's Formulations is not necessarily to coincide with the rotor blade surface. A fictitious surface that is permeable can be used as the integral surface. If this fictitious surface is able to enclose all the noise sources, then the volume integration to find the quadrupole noise can be neglected, significantly reducing the complexity of fully solving the FW-H equation. This idea was already proposed in Ffocws Williams and Hawkings original paper(FfowcsWilliams \& Hall [1970]), however; it would never be implemented until the CFD technology is advanced enough to provide accurate predictions of fluid quantities on the permeable surface. 


\section{LITERATURE SURVEY: MULTI-OBJECTIVE DESIGN AND ANALYSIS FOR A TRANSONIC CENTRIFUGAL COMPRESSOR}

\subsection{Turbomachinery aeroacoustics}

\subsubsection{Turbomachinery noise sources}

Turbomachinery noise has been a very undeseriable phenomenon for a long time. Literature dedicated to this research began as early as the 1900s. Some thorough reviews on the axial turbomachinery noise work were published by Morfey [1973] and later Cumpsty [1977]. Understanding turbomachinery noise field requires a very fine knowledge of the flow field going through turbomachines. The physics of the compressible flow inside turbomachines has strong three dimensionality owing to the blade velocity variations and high blade-twisting. Without powerful computational resources, simulations or modellings for complex flow physics are difficult to accomplish, particularly for transonic turbomachines. Only in the past two decades, both computer hardware and CFD techniques have been constantly improved, which paves the way for the aeroacoustic and aerodynamic research.

In a review paper, Peake \& Parry [2012] emphasised the present impending quest of the public concern to reduce greenhouse emission and aviation noise. A number of targets have been set in both Europe and the USA to cut the $\mathrm{CO}_{2}$, $\mathrm{NO}_{x}$ emssion and significantly reduce aircraft noise.

Turbomachinery noise sources and generation mechanisms must be well understood before better design can be proposed. Cumpsty [1989], Peake \& Parry [2012] categorised the noise sources of turbomachines very similarly. Figure 2.2 shows the sub- and supersonic fan noise spectra. One can see that there are two components in the spectra, namely, tonal and broadband noise. In the subsonic spectrum, it can be seen that the tones at blade passing frequency $(\mathrm{BPF})^{1}$ and its harmonics are dominant, the rest of the spectrum are the broadband noise which is considerably lower. It is, however, quite different a story for the supersonic noise spectrum. The broadband noise is less well-defined and the BPFs noise peaks are mixed up with other pure tones, which are therefore called multiple pure tones, or combination pure tones, or buzz-saw noise as it is heard. Interestingly, the buzz-saw noise is a specific supersonic phenomenon.

The generation mechanisms of tonal and broadband noise are different and

\footnotetext{
${ }^{1} \mathrm{BPF}=$ Rotating frequency $\times$ Number of Blades
} 
2. LITERATURE SURVEY: MULTI-OBJECTIVE DESIGN AND ANALYSIS FOR A TRANSONIC CENTRIFUGAL COMPRESSOR
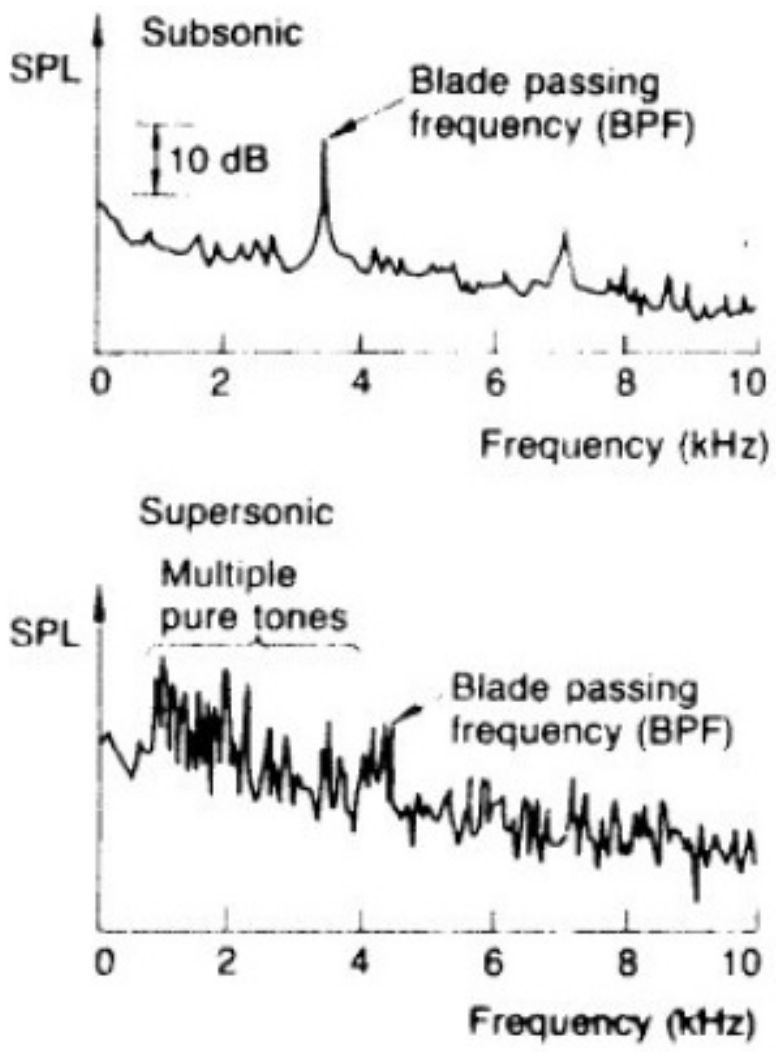

Figure 2.2: Noise spectrum of a transonic fan, Cumpsty [1989] 


\section{LITERATURE SURVEY: MULTI-OBJECTIVE DESIGN AND ANALYSIS FOR A TRANSONIC CENTRIFUGAL COMPRESSOR}

elaborated below.

- Tonal noise

1. Rotor-blade inducing

The tonal components of BPF and its harmonics are highly associated with the rotor-blades that cause the force and volume displacement effects. Provided that a blade row with $B$ blades rotating at the speed of $\omega$ will produce a blade passing frequency $B \omega$. The sound field at the frequency $\omega$ can be decomposed into azimuthal harmonics of orders equal to multiples of $B$. It is worth noting that this mode cannot propagate if the blade tip speed is subsonic, but once the tip speed reaches supersonic conditions, this mode can propagate to the far field. Significantly, the shocks are formed around the blade tips. These shocks attached to the tips eventually cause the buzz-saw noise. This is a primary source noise in transonic turbomachines. The strength of shocks are quite sensitive to the blade geometry and blade-to-blade spatial positions. A careful design of blades can help to reduce the shock strength which is believed to be able to cut the tonal noise eventually.

2. Wake-stator interaction

Another factor causing the tonal noise is the interaction between the wake produced by rotating blades and the stator blade row. This noise is highly related to the numbers of rotor and stator rows, which was first studied by Tyler \& Sorfin [1962].

- Broadband noise

Knowledge of the broadband noise was relatively less available as it is difficult to identify from the spectrum as the tonal noise. However, in an experimental study carried out Ganz et al. [1998], an 18-inch model-scale fan was tested. The obtained results provide many insights for the understanding of this particular noise.

As is shown in Figure 2.2, the broadband noise forms the background for the tonal noise. Cumpsty [1989] suggested two hypotheses for the explanation 


\section{LITERATURE SURVEY: MULTI-OBJECTIVE DESIGN AND ANALYSIS FOR A TRANSONIC CENTRIFUGAL COMPRESSOR}

of the broadband noise generation, 1) interactions between turbulence in the blade passages and blades; 2) flow instabilities in the passages.

1. Turbulence in blade passages

The flow physics in the rotating blade passages is extremely complicated. A great deal of turbulent eddies can be produced near the boundary layer, which can significantly amplify the quadrupole source noise. This mechanism was first studied by Ffowcs-Williams \& Hall [1970].

\section{Blade-tip vortex}

The vortex shedding near the TE(trailing edge) can be formed by the exit flow or the flow in the tip gap shown in Fig. 2.1, which partially originates the broadband noise.

To the best knowledge of the author, the most comprehensive work dedicated to the transonic centrifugal compressor noise measurements is the experimental study by Raitor \& Neise [2008], in which detailed acoustic tests were carried out using two centrifugal compressor stages, which are known as the SRV2 and SRV4 impeller respectively. The SRV2 impeller was coupled with both vaneless and vaned diffuser to measure its noise at both the inlet and outlet. SRV4 is a redesigned impeller to replace with SRV2 in order to gain higher aerodynamic performances. It was only coupled with the vaneless diffuser to the noise measurements. Raitor \& Neise [2008] identified the dominant noise generation mechanisms at different locations, which are summarised in the Table 2.1 and 2.2. Their experimental investigations were the first detailed systematic study conducted for the characterisations of dominant noise generation mechanisms for centrifugal compressors, providing many insights for the understanding and designing of quieter centrifugal compressors. In addition to that, the SRV2 impeller is a well-known case for the aerodynamic studies too. In Chapter 5, we will investigate many more details of this impeller.

An interesting phenomenon reported in their paper is the tip clearance noise at lower operating speeds. This particular noise is very influential at lower speeds and can even be higher than the BPF tones. Previous studies on this particular noise, e.g. Kameier \& Neise [1997] and Fukano \& Jang [2004], based on axial 


\section{LITERATURE SURVEY: MULTI-OBJECTIVE DESIGN AND ANALYSIS FOR A TRANSONIC CENTRIFUGAL COMPRESSOR}

Table 2.1: Dominant mechanisms for subsonic noise generation

\begin{tabular}{ll}
\hline \hline Inlet & TCN (tip clearance noise) at lower impeller tip Mach \\
nutlet & TCN at lower impeller tip Mach numbers $\left(M_{t i p}<0.95\right)$ \\
& in case of compressor with vaneless diffuser, and rotor- \\
& stator-interaction noise in case of vaned diffuser
\end{tabular}

Table 2.2: Dominant mechanisms for supersonic noise generation

\begin{tabular}{ll}
\hline \hline Inlet & Rotor-alone noise at blade passing and its harmonics at \\
& sonic speed Mach numbers; Rotor-alone noise at the BPF \\
& and its harmonics plus buzz-saw noise caused by shock \\
& waves attached to the rotor blades; \\
& Blade tone noise due to rotor-alone noise and rotor-stator \\
interaction noise
\end{tabular}

turbomachines, suggested that the TCN is due to the secondary flow in the gap between the blade tip and casing wall which causes the rotating instability (RI). A mathematical relationship between the tip clearance frequency, blade passing frequency and rotational instability frequency was also given in their paper. In Raitor and Neise's paper they showed that the computed TCN frequency matched the observed one quite well.

\subsubsection{Numerical studies on compressor noise}

In the past two decades, FW-H approach has been extensively used for rotorinduced noise predictions, such as helicopter rotors, wind turbines. However, the noise induced by more complicated turbomachinery rotor configurations, such as a compressor, has been studied less frequently. Numerical acoustic predictions employing this method for centrifugal compressors were carried out only recently and mainly restricted to subsonic conditions, such as Sun \& Lee [2003], Sun et al. [2006], Liu et al. [2006]. These numerical studies suggest that the compressor geometry have strong correlations with the aerodynamic noise field. In order to study the nature of how turbomachine geometries affects the noise field, numerical prediction is a better approach than experimental investigations, since the latter 


\section{LITERATURE SURVEY: MULTI-OBJECTIVE DESIGN AND ANALYSIS FOR A TRANSONIC CENTRIFUGAL COMPRESSOR}

is much more expensive.

Amiet [1988] studied the correlations between the noise of a propeller and its blade and suggested that a swept rotor blade produce less noise than straight rotor blade, since the former would remove singularities on the leading and trailing edges. It is worth mentioning that the effects of the geometry of the vaned-diffuser leading edge on the acoustic field have received much attention. In a series of studies by Ohta and co-workers [2005; 2006; 2009], a new design of centrifugal compressor was proposed. They found that a guide vane with leading tip tapered can significantly help reduce the interaction tone noise and also improve the aerodynamic performance. Zhang et al. [2010] studied a centrifugal fan with inclined vaned diffuser leading edge and showed that introducing a proper inclined angle effectively reduces $3.6 \mathrm{~dB}$ overall noise while improving fan performances.

However, there is still a lack of a consistent design strategy that considers the aerodynamic and aeroacoustic performances for turbomachines, particularly for the transonic ones. It is mainly due to the decoupling of the blade profiling and flow simulations in the conventional design method. If the blade geometry generation and flow physics calculation can be synchronised in an integrated design process, a design strategy may be possibly devised for the multi-objective and multi-disciplinary design. The inverse design method for the turbomachines actually shed the light to such a difficult problem as it can start from the flow field prescriptions and iteratively update the blade profile until the final blade geometry is obtained that can give the specified flow field. In the next section, the inverse design approach for the turbomachines will be reviewed.

\subsection{Inverse design approach for turbomachines}

This section is dedicated to the survey of the inverse design approach for turbomachines. As aforementioned, there has been a long history of this design method and a number of different mathematical formulations including numerical and analytical type with/without taking account of fluid viscosity. Due to the mathematical complexities of this method, it is still often regarded massively as an being-developed method that has not survived the real industrial applications yet, however, to the best knowledge of the author, there are indeed two exceptions, 


\section{LITERATURE SURVEY: MULTI-OBJECTIVE DESIGN AND ANALYSIS FOR A TRANSONIC CENTRIFUGAL COMPRESSOR}

which are known as the TURBOdesign1 and TURBOdesign2 (TURBOdesign1 and TURBOdesign2 will hereinafter referred to as TD1 and TD2) commercial codes. These two codes, especially TD1, have already received significant attention for their capabilities of conducting rapid designs and optimisations.

A brief historical review on the general inverse design method in different forms will be outlined here and it can be shown why TD1 has been selected for the present study.

\subsubsection{Potential flow method}

The earliest inverse design method was developed by Lighthill in 1945. He used the conformal mapping to transform the aerofoil into a circle in a complex plane and prescribed the surface velocity distributions. Stanitz [1953] introduced the potential-stream function method, which resolves the flow dynamics on the $\phi-\psi$ plane and uses flow angle to determine the cascade geometry. The hodograph method developed by Nieuwland [1967] solved the potential equations on the velocity - streamline angle plane. Following that, Sobieczky et al. [1978] employed the fictitious gas method to design aerofoils free of shocks by changing the local density in order to maintain the subsonic regime in the flow field. The major disadvantage of these early inverse methods is the prerequisite of aerofoil thickness that must be very thin or the strict requirement of incompressible flow.

\subsubsection{Circulation method}

The first complete theory of circulation method was reported by Hawthorne et al. [1984]. The blade rows are represented by infinitely thin vorticity sheets on which the average circulation $r \bar{V}_{\theta}$ is prescribed. The flow is assumed to be incompressible and inviscid. Hawthorne et al. [1984] used it to design a 2D cascade. The velocity induced by the bound vorticity is obtained by the Biot-Savart method. Using the non-permeable surface boundary condition, the blade profiles can be iteratively calculated. An alternative way is to use Clebsch formulation to decompose the flow field into a tangentially average and a periodic component. The advantage of Clebsch decomposition is that it allows the inverse method to be easily expanded to be three dimensional, which was subsequently continued by 


\section{LITERATURE SURVEY: MULTI-OBJECTIVE DESIGN AND ANALYSIS FOR A TRANSONIC CENTRIFUGAL COMPRESSOR}

Tan et al. [1984]. He used this method to design the annular cascades. The flow equations are solved by spectral methods in which the velocity is represented by a Fourier series in the tangential direction and a Bessel series in the radial direction. The blade wrap angle is represented by a Chebyshev series in the axial direction and a Cosine series in the radial direction.

Borges [1990a] extended Hawthorne et al. [1984] and Tan et al. [1984]'s work to be adaptable for the incompressible radial turboamchine's design. The flow field is solved by a finite difference scheme and the physical plane is transformed into a body-fitted frame. Borges [1990b] managed to improve the total to static efficiency of a low speed conventionally designed radial turbine by $1.5 \%$.

Soon after the work completed by Borges [1990a], Zangeneh [1991] presented a complete theory that extended it to be able to cope with the compressible flow regimes within either radial, axial and mixed meridional contours. To model the viscosity effect the concept of blockage factor was introduced, which is for the sake of high speed turbomachines that develop thick boundary layer. Zangeneh [1991] used his inverse code for a high speed radial turbine that showed $2.5 \%$ improvement compared to the impeller designed using the conventional approach based on the throughflow theory.

In the subsequent work by Zangeneh [1996], the inverse theory was further developed for impellers with full and splitter blade arrangement. Similarly, Tjokroaminata [1992]; Yang [1991] also extended their inverse design code to design radial turbines with splitter blade. The main difference between Zangeneh [1996] and Tjokroaminata [1992]; Yang [1991] is the way that the blade loading is prescribed.

\subsubsection{Time marching method}

Unlike the circulation method that is of highly analytical nature, the time marching method is more of a numerical one. In general it combines a time marching flow solver (either discretised Euler or Navier-Stokes equations) and blade update algorithm, which iteratively modifies the blade profile based on the difference between the prescribed and a computed flow quantity. It does have the advantage of more accurate solutions over the circulation and potential flow methods, es- 


\section{LITERATURE SURVEY: MULTI-OBJECTIVE DESIGN AND ANALYSIS FOR A TRANSONIC CENTRIFUGAL COMPRESSOR}

pecially for those cases in which the compressibility plays an important role. Depending on the way of prescribing the flow quantity, one can also categorise two different schemes for the time marching method, namely, $\Delta P$ (the difference between the static pressure on the pressure and suction side) and swirl velocity method.

\subsubsection{1 $\Delta P$ method}

The so-to-speak $\Delta P$ method can further be sub-categorised into two schemes depending on the way imposing boundary conditions, which are the transpiration wall and moving wall.

- Transpiration wall method

This method assumes that the blade walls can be treated as permeable and imposes a prescribed flow quantity instead of a zero flux condition. The transpirating flow permeates the blade wall that is iteratively modified until the normal component of the fluxes to the wall vanish to zero. Leonard \& den Braembussche [1992] used this idea and prescribed the Mach number on the 2D blade surface. Their work was later extended by Demeulenaere \& den Braembussche [1998] to a 3D application with the static pressure specified. The difficulty of using this method is the viable static pressure or Mach number needs to be known a priori, otherwise the modifications of the pressure and suction surfaces can result in impossible designs, such as an overlapped or open trailing edge. This problem can be solved by prescribing the pressure difference, or blade loading, on the pressure and suction surface, hence a $\Delta P$ method. Dang et al. [2000]; Medd [2002] demonstrated their results of using such a $\Delta P$ scheme. Boselli [2016] also tried this method for a LP steam turbine design and he reported that this permeable wall boundary condition method has poor robustness.

- Moving wall method

Rather than the permeable wall concept Rooij \& Medd [2012] proposed a reformulated $\Delta P$ method. The blade updating is driven by the difference between any prescribed loadings and the computed ones by the RANS solver. In their formulation, the residue of blade loadings is resolved as a 


\section{LITERATURE SURVEY: MULTI-OBJECTIVE DESIGN AND ANALYSIS FOR A TRANSONIC CENTRIFUGAL COMPRESSOR}

virtual blade velocity, hence, a moving wall method. They used this method to redesign the NASA 37 rotor, but there was no obvious improvement obtained when compared with the early 1990s work using transpiration wall method. Arbabi \& Ghaly [2013] performed similar studies using moving wall idea and coupling with ANSYS CFX flow solver for an axial compressor and turbine stage design, however, the improvement they achieved was also quite small.

In general the $\Delta P$ method is limited to the well-known axial compressor, axial and fan cases whose geometries have quite low three dimensionality. The literature survey did not find any other industrial applications. Particularly, to the best knowledge of the author, no applications of centrifugal turbomachines are available using this $\Delta P$ method.

\subsubsection{Swirl velocity method}

The second category of time marching method is the so-called swirl velocity method, or $r \bar{V}_{\theta}$ method. It is similar to the aforementioned circulation method but the difference is that the flow solution is obtained by the Euler solver instead of a stream function for the average flow component and a potential function for the periodic component. The representative studies of this swirl velocity method were conducted by Dang \& Isgro. [1995] and Tiow et al. [2002].

The difference between the prescribed and calculated swirl velocity, $r \bar{V}_{\theta}$ gives rise to the blade profile updating. It adopts the equation derived by Hawthorne et al. [1984] and Tan et al. [1984] to associate the blade loading with the meridional derivative of $r \bar{V}_{\theta}$.

With an Euler solver used, this swirl velocity method gives more accurate prediction of the flow field in the blade surface and channel. However, the robustness is not as good as the previously mentioned circulation method. In addition to that, there can be quite limited blade loading prescriptions that lead to the converged solutions, which in fact constrains the design space considerably. 


\subsubsection{Summary}

Of all these inverse method reviewed above, the theories proposed by Zangeneh [1991, 1996] have been mostly applied for the industrial applications, and it has been commercialised with the GUI (graphic user interface). The software is now well-known as TURBOdesign1. It has been used for pumps, fans, compressors, diffusers, torque converters, and turbine designs. The success of this theory is driven by its fast design duration and good correlations with the viscous CFD calculations.

\subsection{Existing studies of inversely designed tur- bomachines with acoustic optimisations}

There have already been some studies available using the inverse design method for the acoustic optimisations. For example, Okamoto \& Goto [2009] used TD1 to design and optimise axial box fans and they managed to improve the aerodynamic efficiency by $5.7 \%$ and significantly lowered the noise by $2.6 \mathrm{~dB}(\mathrm{~A})$.

Recently, Stadler et al. [2013] employed TD1, genetic algorithm and LatticeBoltzman method to fully optimise an axial fan to achieve lower noise, higher aerodynamic efficiency and pressure head. They managed to reduce the tonal noise considerably and obtained higher efficiency for a small axial fan. Interestingly, each of their fan blades is individually parametrised, thus, they have different blades for fan to achieve the best aerodynamic and aeroacoustic performances. The experimental measurements confirmed that the variable-geometry fan has considerably lower noise level at the blade passing frequency compared with the identical-geometry fan.

Wang \& Zangeneh [2015] studied the USI7 fan from University of Siegen and redesigned it using TD1. This fan's geometry and acoustic test data were made public by Carolus et al. [2015]. In this paper, it was found that a strong loading at impeller leading edge near the shroud is useful to improve aerodynamic pressure rise, efficiency and reduce noise.

For a multi-objective design and study of a transonic impeller there still remains much space to improve. 


\section{LITERATURE SURVEY: MULTI-OBJECTIVE DESIGN AND ANALYSIS FOR A TRANSONIC CENTRIFUGAL COMPRESSOR}

\subsection{Summary}

In this chapter the description of a turbocharger compressor stage, different types and sources of turbocharger noise, the rotor induced aeroacoustics, sound spectrum of a transonic centrifugal compressor, and different inverse design methods have been reviewed.

It is not difficult to see that there is still a research gap that needs to be fulfilled, which is how to systematically design a transonic centrifugal compressor with improved aerodynamic and aeroacoustic performances. The integration of the inverse design method and aeroacoustic theory provides a possible way to complete such a multi-objective task.

Regarding the compressor noise predictions, a thorough CFD investigation for all noise features in the spectrum accurately predicted is not realistic at this stage for the limited computational resources and affordable time consumptions. Thus, this thesis will only focus on the rotor-related noise, i.e., the tones at blade passing frequency and its harmonics, which are in fact the dominating ones at the inlet of transonic compressors. As to the inverse design approaches, the TD1 code will be employed for this project owing to its robustness and previous success in improving centrifugal machine's performances. The TD2 code has been proven to be effective for some transonic fan designs, but to the best knowledge of the author, there seems to be no one who has reported the success of using this sort of time marching method for centrifugal machine designs.

Therefore, the contribution of completing this thesis is to couple the inverse design method, in this case, TD1 and the aeroacoustic solver for turbomachine rotors to design a transonic centrifugal compressor. The design strategy and procedures will also be rationalised for further turbocharger compressor designs and optimisations. 
"Mathematical reasoning may be regarded rather than schematically as the exercise of a combination of two facilities, which we my call intuition and ingenuity"

Alan Turing (1912 - 1954)

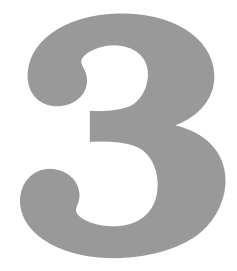

\section{Mathematical formulations for} aeroacoustics

This chapter presents a systematic mathematical formulation for the prediction of aerodynamic noise in both subsonic and supersonic regimes. The most important literature is reviewed.

\subsection{Lighthill's acoustic analogy}

Lighthill's well-known works $[1952 ; 1954 ; 1962]$ have always been marked as the birth of the modern aeroacoustics. By rearranging the continuity and momentum equations, he obtained a wave equation with a source term in the right-hand side, i.e., an inhomogeneous equation, making a connection between fluid mechanics and acoustics. It is called an analogy because this equation only represents a way of how the sound can be represented by a governing equation of a compressible fluid rather than strictly model the acoustics field that is based on the flow-excited 
sound.

Consider an acoustic perturbation with a velocity $\mathbf{u}$ in a stationary medium. The continuity equation in tensor form is,

$$
\frac{\partial \rho}{\partial t}+\frac{\partial\left(\rho u_{i}\right)}{\partial x_{i}}=0
$$

The momentum equation in Reynolds form is,

$$
\frac{\partial\left(\rho u_{i}\right)}{\partial t}+\frac{\partial}{\partial x_{j}}\left(\rho u_{i} u_{j}\right)=-\frac{\partial p}{\partial x_{i}}+\frac{\partial \sigma_{i j}}{\partial x_{j}}
$$

where $\rho$ is the fluid density, $p$ is pressure, $\sigma_{i j}$ is the viscous stress tensor, and

$$
\sigma_{i j}=\mu\left(\frac{\partial u_{i}}{\partial x_{j}}+\frac{\partial u_{j}}{\partial x_{i}}-\frac{2}{3} \delta_{i j} \frac{\partial u_{k}}{\partial x_{k}}\right)
$$

in which $\mu$ is dynamic viscosity and $\delta_{i j}$ is the Kronecker tensor,

$$
\delta_{i j}= \begin{cases}1, & (i=j) \\ 0 . & (i \neq j)\end{cases}
$$

Taking $\frac{\partial}{\partial t}$ of both sides of Eq. 3.1, $\frac{\partial}{\partial x_{i}}$ of both sides of Eq. 3.2 and subtract the latter from the former gives,

$$
\frac{\partial^{2} \rho}{\partial t^{2}}=\frac{\partial^{2} p}{\partial x_{i}^{2}}+\frac{\partial^{2}}{\partial x_{i} \partial x_{j}}\left(\rho u_{i} u_{j}-\sigma_{i j}\right) .
$$

Subtracting $c^{2} \nabla^{2} \rho$ of both sides of Eq. 3.4 yields,

$$
\frac{\partial^{2} \rho}{\partial t^{2}}-c^{2} \nabla^{2} \rho=\frac{\partial^{2} T_{i j}^{\prime}}{\partial x_{i} \partial x_{j}}
$$

Defining that,

$$
T_{i j}^{\prime}=\rho u_{i} u_{j}-\sigma_{i j}+\left(p-c^{2} \rho\right) \delta_{i j},
$$

For a unperturbed flow in far-field, Eq. 3.6 can be simplified to an acoustic 
density wave equation,

$$
\frac{\partial^{2} \rho^{\prime}}{\partial t^{2}}-c^{2} \nabla^{2} \rho^{\prime}=\frac{\partial^{2} T_{i j}}{\partial x_{i} \partial x_{j}},
$$

in which,

$$
\begin{gathered}
\rho^{\prime}=\rho-\rho_{0}, \\
T_{i j}=\rho u_{i} u_{j}-\sigma_{i j}+\left[\left(p-p_{0}\right)-c^{2}\left(\rho-\rho_{0}\right)\right] \delta_{i j},
\end{gathered}
$$

where $T_{i j}$ is the well-known Lighthill stress tensor, and $\rho_{0}$ is the ambient density, $\frac{\partial \rho_{0}}{\partial t}, \frac{\partial^{2} \rho_{0}}{\partial x_{i} \partial x_{j}}$ are both equal to 0.

Eq. 3.8 and 3.10 together give a physical interpretation that "a fluctuating flow in a limited part of an atmosphere otherwise practically at rest, generates the same fluctuations of density as would be produced in a classical stationary acoustic medium by a system of externally applied stress $T_{i j}$ ". (Lighthill [1961]) The right-hand side of Eq. 3.8 is a second spatial derivative, representing distributed quadrupoles of strength $T_{i j}$ per unit volume.

\subsection{Curle's equation}

Lighthill's equation can be understood that the sound field in a real fluid is a quadrupole field if there are no sources of mass injection or externally applied forces on the internal boundaries. Curle [1955] extended Ligthill's work and developed his equation to include the internal boundary effects. It should be mentioned that the standard Kirhhoff formulation (Stratton [1941]) was adopted in Curle's work to solving the inhomogeneous wave equation in an infinite region $V$ outside a fixed stationary internal surface $S$. Curle obtained,

$$
\begin{aligned}
\left.c^{2}(\rho(\boldsymbol{x}, t))-\rho_{0}\right)= & \int_{S}\left[\frac{\partial\left(\rho u_{i} n_{i}\right)}{\partial t}\right]_{\tau} \frac{d S}{4 \pi r}-\frac{\partial}{\partial x_{i}} \int_{S}\left[\left(\rho u_{i} u_{j}+\left(p-p_{0}\right) \delta_{i j}\right) n_{j}\right]_{\tau} \frac{d S}{4 \pi r} \\
& +\frac{\partial^{2}}{\partial x_{i} \partial x_{j}} \int_{V}\left[\frac{T_{i j}}{4 \pi r}\right]_{\tau} d \tau
\end{aligned}
$$




\section{MATHEMATICAL FORMULATIONS FOR AEROACOUSTICS}

where $n_{i}$ is outward normal from $\mathrm{S}, r$ is the distance from an arbitrary point on $S$ to the observer, $[\cdot]_{\tau}$ means that quantities in the square bracket are evaluated at retarded time when the signal is emitted, $\tau=t-r / c$, and $t$ is time when the signal is observed, termed as advanced/observer time.

The important conclusion that can be drawn from Eq. 3.11 is that sound is generated by three parts, 1) sources with strength equal to the rate of variation of local mass outflow from the boundary $S ; 2$ ) dipoles with strength equal to the rate of momentum output from $S ; 3$ ) quadrupoles of strength $T_{i j}$ distributed throughout the flow. The first two terms are surface integrals and the last one is a volume integral.

\subsection{Ffowcs-Willams and Hawkings equation: a convective scheme}

Lighthill and Curle's research shed light on understanding the mechanism of aerodynamic noise generation due to a turbulent flow with/without stationary solid boundaries, and the equations derived suggest good connections between classical fluid dynamics and acoustics. Qualitative and quantitative studies were then performed for the noise predictions. However, neither Lighthill nor Curl's method can be applied in a moving surface. This problem was solved by Ffowcs-Williams \& Hawkings [1969], in which Lighthill and Curl's work was improved to a higher level and the gate to the more advanced modern aeroacoustic studies was opened. In the past few decades, the classical FW-H equation has been extensively studied by numerous researchers to conduct the noise predictions for different rotor configurations in the presence of both subsonic and supersonic cases. The obtained numerical results have shown good reliabilities and the implementations are also of good robustness. However, it should also be mentioned that in the derivation of classical FW-H equation the source surface was assumed to be embodied in a static medium, thus the convective flow effects on the noise field were not modelled. 


\subsubsection{Convective FW-H}

Very recently, Najafi-Yazzdi et al. [2010] developed a more general form of FW-H equation taking account of the presence of a mean flow. A subsequent formulation for solving FW-H has also been derived. This subsection presents the details of the derivation of convective $\mathrm{FW}-\mathrm{H}$ equation and it can be shown that the convective $\mathrm{FW}-\mathrm{H}$ equation is reduced to the normal FW-H expression if the convective flow is absent. The convective $\mathrm{FW}-\mathrm{H}$ equation will be adopted throughout this project for evaluating the noise induced by a centrifugal compressor.

Consider a flow field consisting of a constant moving medium and acoustic perturbation with the velocity of $\mathbf{U}_{\mathbf{0}}{ }^{1}$ and $\mathbf{u}$. The flow has a velocity of $\mathbf{U}_{\mathbf{0}}+$ $\mathbf{u}(\mathbf{x}, t)$ at any arbitrary point. The continuity equation (in tensor form) is,

$$
\frac{\partial \rho}{\partial t}+\frac{\partial \rho\left(U_{0 j}+u_{j}\right)}{\partial x_{j}}=0
$$

which can be simplified to,

$$
\frac{\partial \rho^{\prime}}{\partial t}+U_{0 j} \frac{\partial \rho^{\prime}}{\partial x_{j}}+\frac{\partial \rho u_{j}}{\partial x_{j}}=0
$$

where $\rho=\rho_{0}+\rho^{\prime},[\cdot]^{\prime}$ and $[\cdot]_{0}$ denote the perturbation and ambient quantities respectively. The momentum equation is,

$$
\frac{\partial\left[\rho\left(U_{0 i}+u_{i}\right)\right]}{\partial t}+\frac{\partial}{\partial x_{j}}\left[\rho\left(U_{0 i}+u_{i}\right)\left(U_{0 j}+u_{j}\right)\right]=-\frac{\partial\left(p-p_{0}\right)}{\partial x_{i}}+\frac{\partial \sigma_{i j}}{\partial x_{j}},
$$

which can be rewritten as,

$$
\begin{aligned}
& U_{0 i}\left[\frac{\partial \rho^{\prime}}{\partial t}+U_{0 j} \frac{\partial \rho^{\prime}}{\partial x_{j}}+\frac{\partial \rho u_{j}}{\partial x_{j}}\right]+\frac{\partial\left(\rho u_{i}\right)}{\partial t}+U_{0 j} \frac{\partial\left(\rho u_{i}\right)}{\partial x_{j}} \\
= & -\frac{\partial}{\partial x_{j}}\left[\left(p-p_{0}\right) \delta_{i j}+\rho u_{i} u_{j}-\sigma_{i j}\right] .
\end{aligned}
$$

\footnotetext{
${ }^{1}$ The medium only has the velocity in the translating direction, $\mathbf{U}_{\mathbf{0}}=\left(0,0, U_{0}\right)$
} 


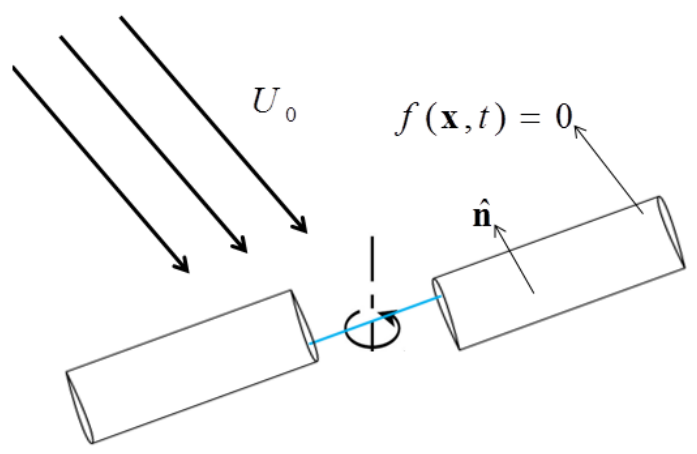

Figure 3.1: Flow over a rigid body whose surface is defined by $f(\mathbf{x}, t)$

Inserting Eq. (2.13) into (2.15) gives,

$$
\frac{\partial\left(\rho u_{i}\right)}{\partial t}+U_{0 j} \frac{\partial\left(\rho u_{i}\right)}{\partial x_{j}}=-\frac{\partial}{\partial x_{j}}\left[\left(p-p_{0}\right) \delta_{i j}+\rho u_{i} u_{j}-\sigma_{i j}\right] .
$$

A closed surface $f(\mathbf{x}, t)=0$ is shown in Figure 3.1. One should notice that this surface, $f(\mathbf{x}, t)$ does not have to coincide with impermeable solid rotor surface. It will be shown later that this surface can be permeable depending on the specific problem. Without losing generality, one can define that:

1. $f(\mathbf{x}, t)>0$ represents the region exterior to the surface;

2. $f(\mathbf{x}, t)<0$ represents the region interior to the surface;

3. $f(\mathbf{x}, t)=0$ represents the closed surface;

and $\|\nabla f\|=1$ on the surface. ${ }^{1}$

One may introduce the Heaviside function,

$$
H(f)= \begin{cases}1, & (f \geq 0) \\ 0 . & (f \neq 0)\end{cases}
$$

The continuity equation for the region exterior to the surface can then be written as

$$
H(f)\left[\frac{\partial \rho^{\prime}}{\partial t}+U_{0 j} \frac{\partial \rho^{\prime}}{\partial x_{j}}+\frac{\partial \rho u_{j}}{\partial x_{j}}\right]=0,
$$

${ }^{1}$ This assumption is not necessary, but it does make the derivation process easier. If $\|\nabla f\| \neq$ 1 , then $f(\mathbf{x}, t)$ can always be redefined by $f(\mathbf{x}, t) /\|\nabla f\|$. 
moving the Heaviside function $H(f)$ into the temporal and spatial differential operators and Eq. 3.18 can be recast as ${ }^{1}$,

$$
\begin{aligned}
& \frac{\partial H(f) \rho^{\prime}}{\partial t}+U_{0 j} \frac{\partial H(f) \rho^{\prime}}{\partial x_{j}}+\frac{\partial H(f) \rho u_{j}}{\partial x_{j}} \\
& =\rho^{\prime} \frac{\partial H(f)}{\partial t}+\rho^{\prime} U_{0 j} \frac{\partial H(f)}{\partial x_{j}}+\rho u_{j} \frac{\partial H(f)}{\partial x_{j}}
\end{aligned}
$$

in which,

$$
\begin{array}{r}
\frac{\partial H(f)}{\partial t}=\frac{d H}{d f} \frac{\partial f}{\partial t}=-v_{j} n_{j} \delta(f)=-v_{n} \delta(f) \\
\frac{\partial H(f)}{\partial x_{j}}=\frac{d H}{d f} \frac{\partial f}{\partial x_{j}}=n_{j} \delta(f)
\end{array}
$$

Two important results have been used in deriving Eq. (2.20) and (2.21), which are

$$
H^{\prime}(f)=\delta(f)
$$

and on the closed surface $f(\boldsymbol{x}, t)=0$

$$
\frac{d f}{d t}=0=\frac{\partial f}{\partial t}+\boldsymbol{v} \nabla f=\frac{\partial f}{\partial t}+v_{n}
$$

Thus, $\frac{\partial f}{\partial t}=-v_{n}$ Combining Eq. (2.19) - (2.21) finally yields,

$$
\frac{\partial\left[H(f) \rho^{\prime}\right]}{\partial t}+U_{0 j} \frac{\partial\left[H(f) \rho^{\prime}\right]}{\partial x_{j}}+\frac{\partial\left[H(f) \rho u_{j}\right]}{\partial x_{j}}=Q_{j} n_{j} \delta(f)
$$

in which,

$$
Q_{j}=\rho\left(u_{j}+U_{0 j}-v_{j}\right)+\rho_{0}\left(v_{j}-U_{0 j}\right)
$$

The same procedure may be applied to the Eq. 3.16 to give

$$
\begin{aligned}
\frac{\partial\left[H(f) \rho u_{i}\right]}{\partial t}+U_{0 j} \frac{\partial\left[H(f) \rho u_{i}\right]}{\partial x_{j}}= & -\frac{\partial}{\partial x_{j}}\left[H(f)\left(\rho u_{i} u_{j}+\left(p-p_{0}\right) \delta_{i j}-\sigma_{i j}\right)\right] \\
& +L_{i j} n_{j} \delta(f)
\end{aligned}
$$

${ }^{1}$ Differentiation of some discontinuous function needs the knowledge of the generalized function theory, for which a very good introductory literature can be found by Farassat [1994; 2000]. 
in which,

$$
L_{i j}=\rho u_{i}\left(u_{j}+U_{0 j}-v_{j}\right)+\left(p-p_{0}\right) \delta_{i j}-\sigma_{i j} .
$$

Similarly, one can do $\frac{\partial}{\partial t}$ (Eq. 3.22) $-\frac{\partial}{\partial x_{i}}$ (Eq. 3.24), which leads to

$$
\begin{aligned}
& \frac{\partial^{2}}{\partial t^{2}}\left[H(f) \rho^{\prime}\right]+U_{0 j} \frac{\partial^{2}}{\partial x_{j} \partial t}\left[H(f) \rho^{\prime}\right]-U_{0 j} \frac{\partial^{2}}{\partial x_{i} \partial x_{j}}\left[H(f) \rho u_{i}\right] \\
= & \frac{\partial}{\partial t}\left[Q_{j} n_{j} \delta(f)\right]+\frac{\partial^{2}}{\partial x_{j} \partial x_{i}}\left[H(f)\left(\rho u_{i} u_{j}+\left(p-p_{0}\right) \delta_{i j}-\sigma_{i j}\right)\right] \\
& -\frac{\partial}{\partial x_{i}} L_{i j} n_{j} \delta(f) .
\end{aligned}
$$

Using Eq. 3.22 to replace the third term in Eq. 3.26, and then subtracting $c^{2} \frac{\partial^{2}}{\partial x_{j}^{2}}\left[H(f) \rho^{\prime}\right]$ at both sides of Eq. 3.26 to obtain the convective $\mathbf{F W}-\mathbf{H}$ equation,

$$
\begin{aligned}
\bar{\square}^{2}\left[H(f) p^{\prime}\right]= & \left(\frac{\partial}{\partial t}+U_{0 j} \frac{\partial}{\partial x_{j}}\right)\left[Q_{i} n_{i} \delta(f)\right]-\frac{\partial}{\partial x_{i}}\left[L_{i j} n_{j} \delta(f)\right] \\
& +\frac{\partial^{2}}{\partial x_{i} \partial x_{j}}\left[H(f) T_{i j}\right]
\end{aligned}
$$

in which,

$$
p^{\prime}=c^{2} \rho^{\prime}
$$

and $T_{i j}=\rho u_{i} u_{j}-\sigma_{i j}+\left[\left(p-p_{0}\right)-c^{2}\left(\rho-\rho_{0}\right)\right] \delta_{i j}$, same as previously defined in Eq. 3.10.

$$
\bar{\square}^{2}=\left[\frac{1}{c^{2}} \frac{\partial^{2}}{\partial t^{2}}-\frac{\partial^{2}}{\partial x_{j}^{2}}+2 \frac{U_{0 j}}{c^{2}} \frac{\partial^{2}}{\partial t \partial x_{j}}+\frac{U_{0 i} U_{0 j}}{c^{2}} \frac{\partial^{2}}{\partial x_{i} \partial x_{j}}\right]
$$

If the medium is at rest, i.e., $U_{0}=0$ then Eq. 3.27 reduces to the classical FW-H equation,

$$
\begin{aligned}
\square^{2}\left[H(f) p^{\prime}\right]= & \frac{\partial}{\partial t}\left[\left(\rho\left(u_{n}-v_{n}\right)+\rho_{0} v_{n}\right) \delta(f)\right]-\frac{\partial}{\partial x_{i}}\left[\Delta P_{i j} n_{j} \delta(f)\right] \\
& +\frac{\partial^{2}}{\partial x_{i} \partial x_{j}}\left[H(f) T_{i j}\right],
\end{aligned}
$$

where $\Delta P_{i j}=\rho u_{i}\left(u_{j}-v_{j}\right)+\left(p-p_{0}\right) \delta_{i j}-\sigma_{i j}$, and Eq. 3.29 becomes the normal 
wave operator,

$$
\square^{2}=\frac{1}{c^{2}} \frac{\partial^{2}}{\partial t^{2}}-\frac{\partial^{2}}{\partial x_{j}^{2}} .
$$

This convective FW-H equation interprets the sound field the same as the nonconvective FW-H equation. The first two terms on the right-hand side of Eq. 3.27 are regarded as thickness/monople and loading/dipole noise, respectively, of which the former is due to the displacement of the fluid by the rotor and the latter due to the unsteady force distribution on the body surface. The last term in Eq. 3.27 is known as the quadrupole noise that is due to the local sound speed variation and finite fluid velocity near the blade. Extensive research has been done to study the characteristics of these three noise sources. The quadrupole noise is found to be negligible for the subsonic rotor noise predictions, but it can become very significant source of pressure fluctuations at high subsonic or transonic speeds. The direct evaluation of the quadrupole source is quite complicated and computationally demanding. If the FW-H equation is used with a permeable data surface that encloses the acoustic sources, then one can avoid the volume integration of quadrupole noise. Detailed discussions will be given later.

\subsection{Integral formulations}

The classical FW-H equation 3.30 has the key feature that it is an inhomogeneous wave equation problem for the external flow embedded in unbounded space, and it can be solved by using the free space Green's function

$$
G(\boldsymbol{x}, t ; \boldsymbol{y}, \tau)=\frac{\delta(\tau-t+r / c)}{4 \pi r}
$$

in which $\boldsymbol{x}$ and $\boldsymbol{y}$ are observer and source coordinates, $\tau$ is the signal emitting time, also known as retarded/source time, and $t$ is the observer time, also known as advanced/reception time. These techniques were used in deriving the wellknown Farassat Formulation 1 and 1A. As Eq. 3.27 includes the convective term, a little modification is introduced. It is assumed without losing generality that the mean flow is along the positive $x_{3}$-direction. A 3-dimensional free-space Green's 
function for the convective equation given by Blokhintsev [1956] is

$$
G(\boldsymbol{x}, t ; \boldsymbol{y}, \tau)=\frac{\delta(\tau-t+r / c)}{4 \pi r^{*}},
$$

where

$$
\begin{gathered}
r=\frac{-M_{0}\left(x_{3}-y_{3}\right)+r^{*}}{\beta^{2}}, \\
r^{*}=\sqrt{\beta^{2}\left[\left(x_{1}-y_{1}\right)^{2}+\left(x_{2}-y_{2}\right)^{2}\right]+\left(x_{3}-y_{3}\right)^{2}}, \\
M_{0}=\frac{U_{0}}{c}, \\
\beta=\sqrt{1-M_{0}^{2}} .
\end{gathered}
$$

Using the above Green's function yields the solution to the convective FW-H equation (Eq. 3.27),

$$
\begin{aligned}
c^{2} \rho^{\prime}(\mathbf{x}, t)= & {\left[\frac{\partial}{\partial t}+U_{0} \frac{\partial}{\partial x_{3}}\right] \int_{-\infty}^{t} \int_{V} Q_{i} n_{i} \delta(f) \frac{\delta(g)}{4 \pi r^{*}} d^{3} \mathbf{y} d \tau } \\
& -\frac{\partial}{\partial x_{i}} \int_{-\infty}^{t} \int_{V}\left[L_{i j} n_{j} \delta(f)\right] \frac{\delta(g)}{4 \pi r^{*}} d^{3} \mathbf{y} d \tau \\
& +\frac{\partial^{2}}{\partial x_{i} \partial x_{j}} \int_{-\infty}^{t} \int_{V}\left[H(f) T_{i j}\right] \frac{\delta(g)}{4 \pi r^{*}} d^{3} \mathbf{y} d \tau,
\end{aligned}
$$

in which

$$
g=\tau-t+\frac{r}{c},
$$

representing a sphere centred at the observer position $\boldsymbol{x}$ with a radius of the distance between the $\boldsymbol{x}$ and $\boldsymbol{y}$.

Eq. 3.38 can be simplified to the same form as Eq. (37) in the paper by Brentner \& Farassat [2003]. The first term and second term on the RHS of Eq. 3.38 are the thickness noise and loading noise respectively, and the third one is quadrupole noise. In order to perform the integration of the Dirac delta functions $\delta(f)$ and $\delta(g)$, the current variables must be changed. Brentner \& Farassat [2003] suggested three different ways that can eventually give three different inte- 


\section{MATHEMATICAL FORMULATIONS FOR AEROACOUSTICS}

gral algorithms, termed as retarded-time algorithm, collapsing sphere algorithm, and emission surface algorithm, of which the first one is used most commonly. The well-known Farassat Formulation 1A exploited the retarded-time algorithm and it is particularly suitable for subsonic noise predictions. The latter two algorithms can be employed for the supersonic noise prediction, but the numerical implementations are of great complexities.

\subsection{Summary}

This chapter presented a detailed set of derivations of state-of-the-art equations for aerodynamic noise generation mechanism. Starting from the first principle of continuity and momentum equation, Lighthill pioneered the modern aerodynamic sound theory. The sound due to some small perturbed flow in a still medium is modeled by an inhomogeneous wave equation with a source term $T_{i j}$ on the right-hand. This work was later extended by Curle to include the stationary solid boundaries and furthered by Ffowcs-Williams and Hawkings. In their classical paper published in 1969, the solid moving boundaries were introduced and a more general acoustic analogy equation was derived. This FW-H equation is generally regarded as the foundation for modern rotor-noise prediction problems. Since the mid 1970s, Farassat have been actively working in this area and developed many approaches for the practical noise computations. Recently, Najafi-Yazdi has extended Farassat's formulations to include the effects due to some mean background flow. This is particulary suitable for noise predictions due to fans or compressors. The next chapter will discuss the details of integral formulations for solving the differential FW-H equation and various numerical algorithms that can be used in both subsonic and supersonic regimes. 
"There are two ways of constructing a software design: One way is to make it so simple that there are obviously no deficiencies, and the other way is to make it so complicated that there are no obvious deficiencies. The first method is far more difficult."

Sir C.A.R. Hoare (1934 - present)

"Measuring programming progress by lines of code is like measuring aircraft building progress by weight."

Bill Gates (1955 - present)

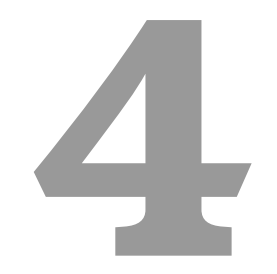

\section{Aeroacoustic solver for rotor-induced}

noise

This chapter describes the integral formulations and numerical algorithms for evaluating the rotor-noise in practice. Both of the subsonic and supersonic scenarios will be considered.

\subsection{Integral formulations}

It can be seen from Eq. 3.38 two Dirac delta functions $\delta(f)$ and $\delta(g)$ have been introduced to the integrand when using the Green's function technique. As has been mentioned in the preceding chapter, three ways of variable changes proposed by Brentner \& Farassat [2003] can be exploited.

Consider an inhomogeneous wave equation

$$
\square^{2} \phi(\boldsymbol{x}, t)=\psi(\boldsymbol{x}, t) \delta(f)
$$


Applying the free space Green's function $\delta(g) / 4 \pi r$ to Eq. 3.1 yields an integral representation

$$
4 \pi \phi(\boldsymbol{x}, t)=\int_{-\infty}^{t} \int_{-\infty}^{\infty} \frac{\psi(\boldsymbol{x}, t) \delta(f) \delta(g)}{r} d \boldsymbol{y} d \tau
$$

Eq. (3.2) can be re-expressed as

$$
\begin{aligned}
4 \pi \phi(\boldsymbol{x}, t) & =\int_{f=0}\left[\frac{\psi(\boldsymbol{x}, t)}{r\left(1-M_{R}\right)}\right]_{\tau} d S, \\
4 \pi \phi(\boldsymbol{x}, t) & =\int_{-\infty}^{t} \int_{f=0} \frac{\psi(\boldsymbol{x}, t)}{r \sin \vartheta} c d \Gamma d \tau, \\
4 \pi \phi(\boldsymbol{x}, t) & =\int_{f=0} \frac{1}{r}\left[\frac{\psi(\boldsymbol{x}, t)}{\Lambda}\right]_{\tau} d \Sigma
\end{aligned}
$$

with the variable transformations:

1. $\left(y_{3}, \tau\right) \rightarrow(f, g)$

$$
\begin{aligned}
d \boldsymbol{y} d \tau & =\frac{d y_{1} d y_{2} d f d g}{\partial(f, g) / \partial\left(y_{3}, \tau\right)}=\frac{d y_{1} d y_{2} d f d g}{\left(\partial f / \partial y_{3}\right)(\partial g / \partial \tau)} \\
& =\frac{d S d f d g}{1-M_{R}}
\end{aligned}
$$

in which

$$
\begin{aligned}
g & =\tau-t+r / c \\
\frac{d g}{d \tau} & =1-\frac{1}{c} \frac{\partial y_{i}}{\partial \tau} \frac{\partial r^{*}}{\partial y_{i}}=1-\frac{v_{i}}{c} r_{i}^{*}=1-M_{R} \\
d S & =\frac{d y_{1} d y_{2}}{\partial f / \partial y_{3}}=\frac{d y_{1} d y_{2}}{\nabla f \hat{e}_{3}}
\end{aligned}
$$

2. $\left(y_{2}, y_{3}\right) \rightarrow(f, g)$ 


$$
\begin{aligned}
d \boldsymbol{y} & =\frac{d y_{1} d f d g}{\partial(f, g) / \partial\left(y_{2}, y_{3}\right)}=\frac{d y_{1} d f d g}{(\nabla f \times \nabla g) \cdot \hat{x}_{1}} \\
& =\frac{c d y_{1} d f d g}{(\nabla f \times \nabla r) \cdot \hat{e}_{1}}=\frac{c d \Gamma d f d g}{(\hat{\boldsymbol{n}} \times \hat{\boldsymbol{r}}) \cdot \hat{e}_{1}} \\
& =\frac{c d \Gamma d f d g}{\sin \vartheta},
\end{aligned}
$$

The geometrical visualisation of $g, \hat{\boldsymbol{n}}, \hat{\boldsymbol{r}}, \Gamma, \vartheta$ can be found in Figure 4.1.

3. $\left(y_{3}, \tau\right) \rightarrow(F, g)$

$$
\begin{aligned}
d \boldsymbol{y} d \tau & =\frac{d y_{1} d y_{2} d F d g}{\partial(F, g) / \partial\left(y_{3}, \tau\right)}=\frac{d y_{1} d y_{2} d F d g}{\partial F / \partial y_{3}} \\
& =\frac{d y_{1} d y_{2} d F d g}{\nabla F \cdot\left(N_{3} \hat{e}_{3}\right)} \\
& =\frac{d y_{1} d y_{2} d F d g}{\|\nabla F\| \cdot\left\|\hat{N}_{3}\right\| \cos \psi} \\
& =\frac{d \Sigma d F d g}{\Lambda}
\end{aligned}
$$

where the function $F$ is defined as $F(\boldsymbol{y} ; \boldsymbol{x}, t)=f(\boldsymbol{y}, t-r / c),\|\nabla F\|=\Lambda$, $N_{3}$ is the third component of the unit normal vector the surface $F=0$, $d \Sigma=\frac{d y_{1} d y_{2}}{\left\|\hat{N}_{3}\right\| \cos \psi}$, and $\psi$ is the angle between $F$ surface normal vector and $\hat{e}_{3}$.

These three different variable changes can eventually give three different formulations, namely, retarded-time, collapsing sphere and emission surface algorithms. The physical interpretations of quantities will be given later.

\subsection{Integral solution to the convective FW-H equation}

The integral formulation (Eq. 3.38) to the convective FW-H equation is very challenging for conducting numerical evaluations due to the occurrence of temporal and spatial derivatives. This section presents the details of the ways of 


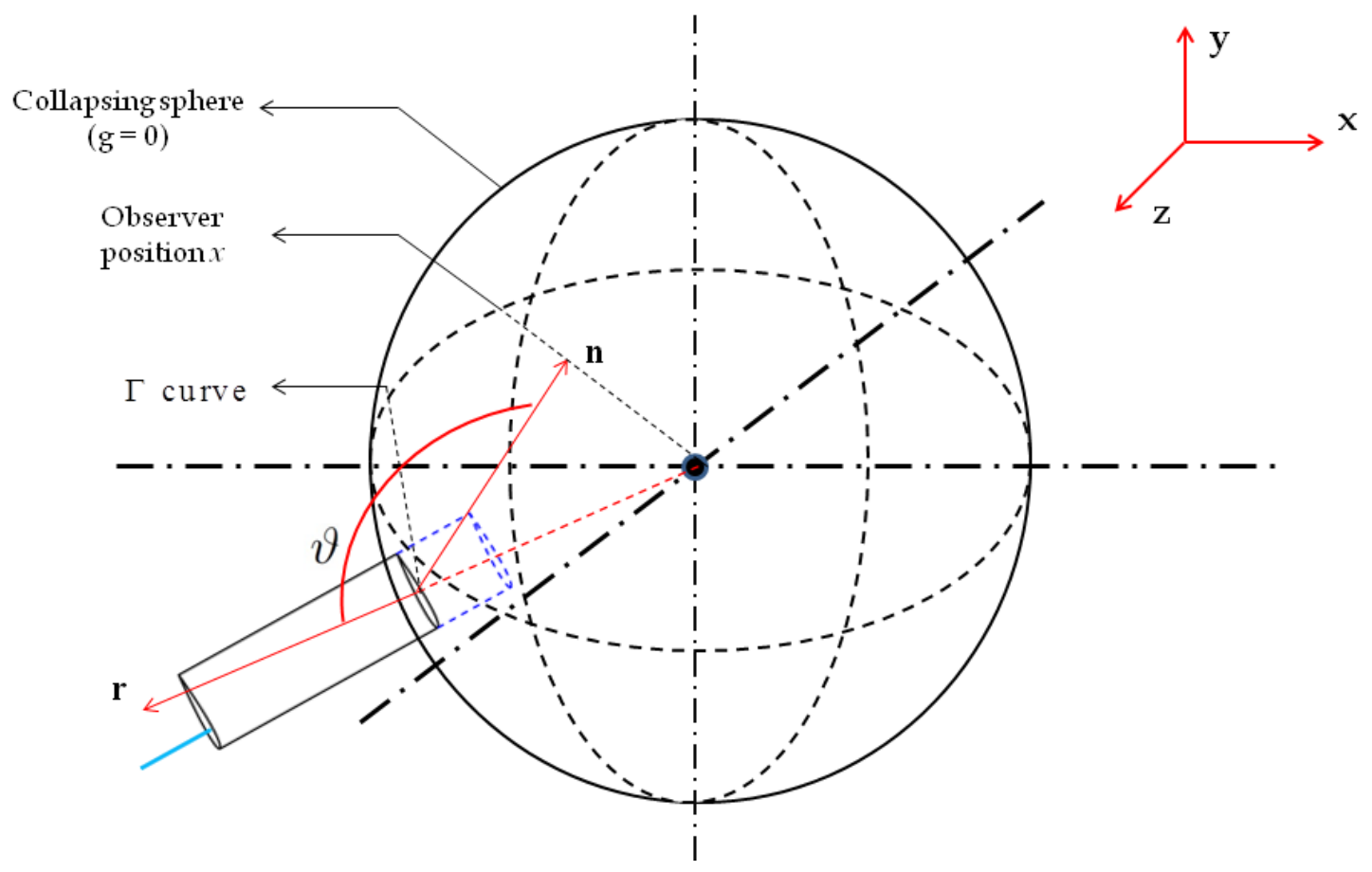

Figure 4.1: Schematics of collapsing sphere and $\Gamma$ curve 


\section{AEROACOUSTIC SOLVER FOR ROTOR-INDUCED NOISE}

circumventing this problem using the aforementioned three different algorithms.

As will be shown, the quadrupole noise term can be omitted by using a permeable surface within which all the noise sources are included. Integral representations for both thickness and loading noise will be given for further numerical implementations.

\subsubsection{Retarded-time algorithm}

\subsubsection{Thickness noise}

From Eq. 3.38, the thickness noise, $p_{T}^{\prime}$, is,

$$
4 \pi p_{T}^{\prime}(\mathbf{x}, t)=\left[\frac{\partial}{\partial t}+U_{0} \frac{\partial}{\partial x_{3}}\right] \int_{-\infty}^{t} \int_{V} Q_{i} n_{i} \delta(f) \frac{\delta(g)}{r^{*}} d^{3} \mathbf{y} d \tau
$$

where the spatial derivative $\frac{\partial}{\partial x_{3}}$ will be converted into a temporal derivative for numerical implementation.

One may consider,

$$
\frac{\partial}{\partial x_{i}}\left[\frac{\delta(g)}{r^{*}}\right]=\frac{1}{c} \frac{\partial r}{\partial x_{i}} \frac{\delta^{\prime}(g)}{r^{*}}-\frac{\partial r^{*}}{\partial x_{i}} \frac{\delta(g)}{r^{* 2}}
$$

and define that

$$
\begin{gathered}
\tilde{r}=\frac{\partial r}{\partial x_{i}} \\
\tilde{r}^{*}=\frac{\partial r^{*}}{\partial x_{i}}
\end{gathered}
$$

Recalling Eq. 3.34 and 3.35, one can get

$$
\begin{aligned}
\frac{\partial r}{\partial x_{i}} & =\left(\frac{x_{1}-y_{1}}{r^{*}}, \frac{x_{2}-y_{2}}{r^{*}}, \frac{1}{\beta^{2}}\left(-M_{0}+\frac{x_{3}-y_{3}}{r^{*}}\right)\right), \\
\frac{\partial r^{*}}{\partial x_{i}} & =\left(\frac{\beta^{2}\left(x_{1}-y_{1}\right)}{r^{*}}, \frac{\beta^{2}\left(x_{2}-y_{2}\right)}{r^{*}}, \frac{x_{3}-y_{3}}{r^{*}}\right) .
\end{aligned}
$$

Then,

$$
\frac{\partial}{\partial x_{i}}\left[\frac{\delta(g)}{r^{*}}\right]=\frac{1}{c} \frac{\tilde{r}_{i} \delta^{\prime}(g)}{r^{*}}-\frac{\tilde{r}_{i}^{*} \delta(g)}{r^{* 2}},
$$


where,

$$
\frac{\partial}{\partial t}\left[\frac{\tilde{r}_{i} \delta(g)}{r^{*}}\right]=-\frac{\tilde{r}_{i} \delta^{\prime}(g)}{r^{*}} .
$$

Combining, Eq. 4.11 to 4.16

$$
\frac{\partial}{\partial x_{i}}\left[\frac{\delta(g)}{r^{*}}\right]=-\frac{1}{c} \frac{\partial}{\partial t}\left[\frac{\tilde{r}_{i} \delta(g)}{r^{*}}\right]-\frac{\tilde{r}^{*} \delta(g)}{r^{* 2}}
$$

Thus, Eq. 4.10 can be rewritten as

$$
\begin{aligned}
4 \pi p_{T}^{\prime}(\mathbf{x}, t)= & \frac{\partial}{\partial t} \int_{-\infty}^{t} \int_{V} Q_{i} n_{i} \delta(f) \frac{\delta(g)}{r^{*}} d^{3} \mathbf{y} d \tau \\
& -M_{0} \frac{\partial}{\partial t} \int_{-\infty}^{t} \int_{V} Q_{j} n_{j} \delta(f) \frac{\tilde{r}_{3} \delta(g)}{r^{*}} d^{3} \mathbf{y} d \tau \\
& -U_{0} \int_{-\infty}^{t} \int_{V} Q_{j} n_{j} \delta(f) \frac{\tilde{r}_{3}^{*} \delta(g)}{r^{* 2}} d^{3} \mathbf{y} d \tau
\end{aligned}
$$

Applying the change of variable defined by Eq. 4.6 to Eq. 4.18 results in the retarded-time formulation

$$
\begin{aligned}
4 \pi p_{T}^{\prime}(\boldsymbol{x}, t)= & \frac{\partial}{\partial t} \int_{f=0}\left[\frac{Q_{j} n_{j}}{r^{*}\left(1-M_{R}\right)}\right]_{\tau} d S-M_{0} \frac{\partial}{\partial t} \int_{f=0}\left[\frac{\tilde{r}_{3} Q_{j} n_{j}}{r^{*}\left(1-M_{R}\right)}\right]_{\tau} d S \\
& -U_{0} \int_{f=0}\left[\frac{\tilde{r}_{3}^{*} Q_{j} n_{j}}{r^{* 2}\left(1-M_{R}\right)}\right]_{\tau} d S
\end{aligned}
$$

in which $d S$ is the elementary area on $f=0$ and the subscript $\tau^{1}$ indicates that the quantities in the square bracket are evaluated at retarded time. Eq. 4.19 can be reduced to the thickness noise integral of Farassat Formulation 1 if the mean flow is stationary and $f=0$ is chosen to be the blade surface.

As occurred in Farasssat Fomrulation 1, one of the main difficulties in numerical evaluation of the above integral representation is the observer-time derivative, $\frac{\partial}{\partial t}$ outside the integral. This inconvenience can be removed by moving the observer temporal derivative inside the integral and transforming it into a sourcetime derivative, $\frac{\partial}{\partial \tau}$. This mathematical manipulation is complex and a detailed

\footnotetext{
${ }^{1} \tau=t-r / c$, here $r$ is defined in Eq. 3.34
} 


\section{AEROACOUSTIC SOLVER FOR ROTOR-INDUCED NOISE}

derivation can be found in Appendix A.

$$
\begin{aligned}
4 \pi p_{T}^{\prime}(\boldsymbol{x}, t)= & \int_{f=0}\left[\frac{\dot{Q}_{i} n_{i}+Q_{i} \dot{n}_{i}}{r^{*}\left(1-M_{R}\right)^{2}}\right]_{\tau} d S+\int_{f=0}\left[-\frac{\partial r^{*}}{\partial \tau} \frac{Q_{i} n_{i}}{r^{* 2}\left(1-M_{R}\right)^{2}}\right]_{\tau} d S \\
& +\int_{f=0}\left[\frac{\partial M_{R}}{\partial \tau} \frac{Q_{i} n_{i}}{r^{*}\left(1-M_{R}\right)^{3}}\right]_{\tau} d S \\
& -M_{0} \int_{f=0}\left[\frac{\tilde{r}_{3} \dot{Q}_{i} n_{i}+\tilde{r}_{3} Q_{i} \dot{n}_{i}+\dot{\tilde{r}}_{3} Q_{i} n_{i}}{r^{*}\left(1-M_{R}\right)^{2}}\right]_{\tau} d S \\
& +M_{0} \int_{f=0}\left[\frac{\partial r^{*}}{\partial \tau} \frac{\tilde{r}_{3} Q_{i} n_{i}}{r^{* 2}\left(1-M_{R}\right)^{2}}\right]_{\tau} d S \\
& -M_{0} \int_{f=0}\left[\frac{\partial M_{R}}{\partial \tau} \frac{\tilde{r}_{3} Q_{i} n_{i}}{r^{*}\left(1-M_{R}\right)^{3}}\right]_{\tau} d S \\
& -U_{0} \int_{f=0}\left[\frac{\tilde{r}_{3}^{*} Q_{i} n_{i}}{r^{* 2}\left(1-M_{R}\right)}\right]_{\tau} d S
\end{aligned}
$$

Eq. 4.20 is the integral representation of thickness noise, which was first derived by Najafi-Yazzdi et al. [2010]. This equation can be reduced to the thickness noise representation of the well-known Farassat Formulation 1A.

\subsubsection{Loading noise}

An integral formulation for the loading noise can be obtained using the same procedure used for the thickness noise. Recall Eq. 3.38, the loading noise attributes to the second term on the RHS,

$$
4 \pi p_{L}^{\prime}(\boldsymbol{x}, t)=-\frac{\partial}{\partial x_{i}} \int_{-\infty}^{t} \int_{V}\left[L_{i j} n_{j} \delta(f)\right] \frac{\delta(g)}{r^{*}} d^{3} \mathbf{y} d \tau
$$

As what has been done for Eq. 4.10, the spatial derivative must be converted into the temporal derivative. Then, the variables $\left(y_{3}, \tau\right)$ are transformed into $(f, g)$. Using Eq. 4.6 and 4.17, Eq. 4.21 is recast as

$$
4 \pi p_{L}^{\prime}(\boldsymbol{x}, t)=\frac{1}{c} \frac{\partial}{\partial t} \int_{f=0}\left[\frac{L_{i j} n_{j} \tilde{r}_{i}}{r^{*}\left(1-M_{R}\right)}\right]_{\tau} d S+\int_{f=0}\left[\frac{L_{i j} n_{j} \tilde{r}_{i}^{*}}{r^{* 2}\left(1-M_{R}\right)}\right]_{\tau} d S .
$$

It can be found that the loading noise in Farasat Formulation 1 can be recovered if $U_{0}=0$ and the integral surface is chosen to coincide with the rotor blade surface. 


\section{AEROACOUSTIC SOLVER FOR ROTOR-INDUCED NOISE}

The observer-time derivative can be moved inside the integral and replaced with source-time derivative, the loading noise integral representation can be recast as

$$
\begin{aligned}
4 \pi p_{L}^{\prime}(\boldsymbol{x}, t)= & \frac{1}{c} \int_{f=0}\left[\frac{\tilde{r}_{i} \dot{L}_{i j} n_{j}+\tilde{r}_{i} L_{i j} \dot{n}_{j}+\dot{\tilde{r}}_{i} L_{i j} n_{j}}{r^{*}\left(1-M_{R}\right)^{2}}\right]_{\tau} d S \\
& -\frac{1}{c} \int_{f=0}\left[\frac{\partial r^{*}}{\partial \tau} \frac{L_{i j} n_{j} \tilde{r}_{i}}{r^{* 2}\left(1-M_{R}\right)^{2}}\right]_{\tau} d S \\
& +\frac{1}{c} \int_{f=0}\left[\frac{\partial M_{R}}{\partial \tau} \frac{L_{i j} n_{j} \tilde{r}_{i}}{r^{*}\left(1-M_{R}\right)^{3}}\right]_{\tau} d S \\
& +\frac{1}{c} \int_{f=0}\left[\frac{L_{i j} n_{j} \tilde{r}_{i}^{*}}{r^{* 2}\left(1-M_{R}\right)}\right]_{\tau} d S .
\end{aligned}
$$

Eq. 4.23 can also be reduced to the loading noise part in Farassat Formulation $1 \mathrm{~A}$ by neglecting the mean flow and integrating on the blade surface. Eq.4.20 and 4.23 are named as Formulation 1C by Najafi-Yazzdi et al. [2010].

\subsubsection{Mid-panel quadrature}

The most common method of numerically evaluating the retarded-time integrals is to use the mid-panel quadrature method. Suppose Eq. 4.20 and 4.23 can be recast in the form,

$$
4 \pi p^{\prime}(\mathbf{x}, t)=\int_{f=0}\left[\frac{I(\mathbf{y}, \tau)}{r^{* \alpha_{1}}\left(1-M_{R}\right)^{\alpha_{2}}}\right]_{\tau} d S
$$

where $I(\mathbf{y}, \tau)$ is the re-organized source strength function depending on source position and time, and $\alpha_{1}, \alpha_{2}$ integers which depend on the kind of source, such as, monopole, dipole. It can be approximated as,

$$
4 \pi p^{\prime}(\mathbf{x}, t) \approx \sum_{i=1}^{N}\left[\frac{I_{i}\left(y_{i}, t-r_{i}^{*} / c\right)}{r_{i}^{* \alpha_{1}}\left(1-M_{R_{i}}\right)^{\alpha_{2}}}\right]_{\tau} \delta S_{i}
$$

The surface $S$ is divided into $\mathrm{N}$ panels and the integrand $I_{i}$ is evaluated at the centre of every panel, $y_{i}$ at the retarded time. 


\section{AEROACOUSTIC SOLVER FOR ROTOR-INDUCED NOISE}

\subsubsection{Root-find algorithm}

An algorithm to find the retarded times is described in this section. Subsonic and supersonic regimes will be both addressed.

$$
c(t-\tau)=r=\|\boldsymbol{x}-\boldsymbol{y}\|
$$

The physical interpretation of Eq. 4.26 is that a sound signal emitted at time $\tau$ travels a distance of $r$ over a period of time $t-\tau$, where $t$ is the time when the observer receives the signal, $\boldsymbol{x}$ is the position vector of the observer, and $\boldsymbol{y}$ the position vector of a panel source. Recall Eq. 3.34, one can get

$$
\begin{aligned}
& \beta^{4} c^{2}(\tau-t)^{2}+M_{0}^{2}\left(x_{3}-y_{3}\right)^{2}-2 c M_{0} \beta^{2}(\tau-t) \\
& =\beta^{2}\left[\left(x_{1}-y_{1}\right)^{2}+\left(x_{2}-y_{2}\right)^{2}\right]+\left(x_{3}-y_{3}\right)^{2} .
\end{aligned}
$$

Suppose that the observer is stationary and the rotor source has no translation velocity but only rotational speed $\omega$,

$$
\begin{aligned}
& y_{1}=R_{0} \cos (\omega \tau+\theta), \\
& y_{2}=R_{0} \sin (\omega \tau+\theta),
\end{aligned}
$$

where $\theta$ is

$$
\theta=\tan ^{-1} \frac{y_{2}}{y_{1}}
$$

the initial phase angle, and

$$
R_{0}=\sqrt{y_{1}^{2}+y_{2}^{2}}
$$

Introducing $\phi=\tau-t$, Eq. 4.27 yields

$$
\beta^{4} c^{2} \phi^{2}-2 c M_{0} \beta^{2} \phi+A=B \cos (\omega(\phi+t)+\theta)+C \sin (\omega(\phi+t)+\theta),
$$




\section{AEROACOUSTIC SOLVER FOR ROTOR-INDUCED NOISE}

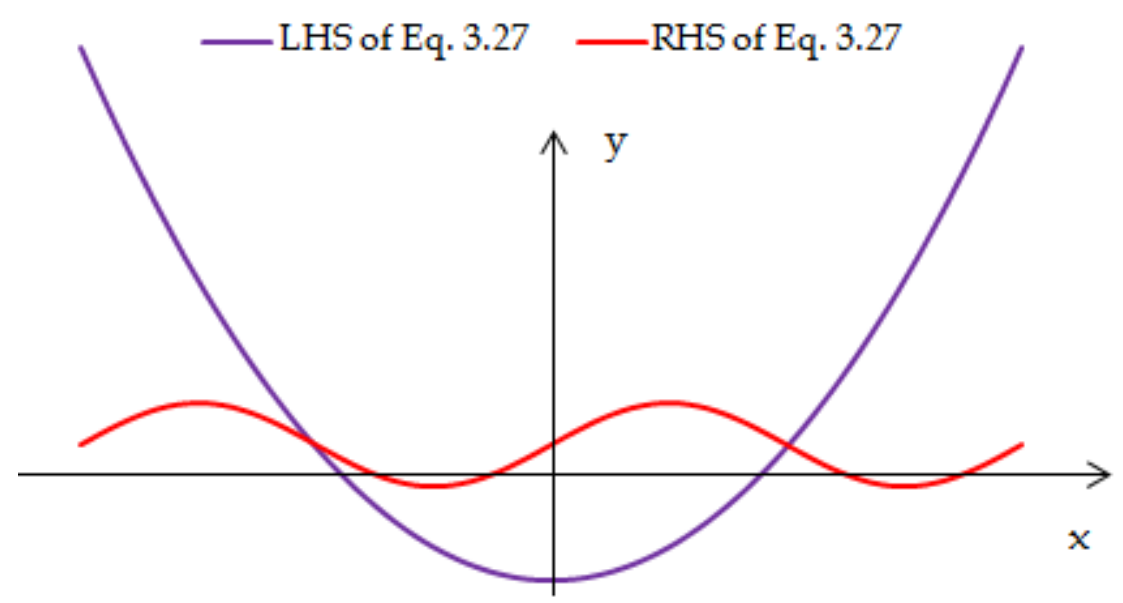

Figure 4.2: Roots of the retarded time equation

where

$$
\begin{aligned}
& A=\beta^{2}\left(x_{1}^{2}+x_{2}^{2}+R_{0}^{2}\right)+\left(M_{0}^{2}-1\right)\left(x_{3}-y_{3}\right)^{2}, \\
& B=-2 x_{1} R_{0}, \\
& C=-2 x_{2} R_{0} .
\end{aligned}
$$

In the retarded-time scheme, the time series of the reception time are given with a constant interval and the corresponding emission time for each panel and each time step is calculated using Eq. 4.31. This is the most computationally consuming part and it can be solved by using the Newton-Raphson method very efficiently and accurately. Figure. 4.2 shows a generic example of roots finding of the retarded time equation. The parabola and sinusoid stand for the LHS and RHS of Eq. 4.31 respectively. It can be seen that there are two intersections of these two curves, indicating two roots of Eq. 4.31. If there is only occurrence of subsonic motion, $\phi$ cannot be greater than zero and only the negative root is accepted. ${ }^{1}$

\footnotetext{
${ }^{1}$ If $\phi>0$, then $\tau>t$. This means that one can perceive sound before its emission, which is obviously impossible.
} 


\section{AEROACOUSTIC SOLVER FOR ROTOR-INDUCED NOISE}

\subsubsection{Drawbacks of retarded-time algorithm}

Eq. 4.20 and 4.23 are both surface integrations and robust for numerical implementations. However, there are two important drawbacks in these two formulations, which are particularly effective in dealing with noise radiation from supersonic sources.

1. Multiple-roots of retarded-time equation

In the presence of supersonic motions, one source point can emit signals at different times and those signals arrive at the observer simultaneously. This means that the retarded-time equation can have more than one root, giving more trouble to the Newton-Raphson root-finder.

2. Doppler singularity

The multiple-roots can be solved with more care in spite of some extra complexities. The singularity in the denominators of the integrands of thickness and loading formulations is the real problem. Under supersonic source conditions, the relative Mach number $M_{R}$ can be equal to 1 , giving rise to the ultimate failure of retarded-time method.

\subsubsection{Source-time dominant algorithm}

An alternative method has been proposed by Casalino [2003] to replace the retarded-time algorithm so that the multiple-roots problem can be avoided. Instead of selecting the observer times in advance, the source time series can be prescribed first and then determine when the observer would receive the signals. The advanced/reception time is directly calculated using Eq. 4.33, and every single $\tau$ only corresponds to single $t$. However, a sequence of equally spaced source times would not yield a sequence of equally spaced observer times. As Eq. 4.33 suggests

$$
t=\tau+\frac{r(\tau)}{c}
$$

$t$ and $\tau$ do not hold a linear relationship due to the term, $r(\tau) / c$. The observer time history needs to be interpolated to provide the contribution at desired observer times in order for further summations. This algorithm has been adopted by several researchers, Lyrintzis \& Xue [1997] and Casalino [1996]. Bres et al. [2004] 


\section{AEROACOUSTIC SOLVER FOR ROTOR-INDUCED NOISE}

analysed the efficiency of these two algorithms and identified that the source-time dominant algorithm is less computational demanding for manoeuvring rotors.

In the next two subsections, another two algorithms that can applied to avoid the Doppler singularity will be discussed in some detail.

\subsubsection{Collapsing sphere algorithms}

In developing Eq. 4.20 and 4.23, the frame transformation $\left(y_{3}, \tau\right) \rightarrow(f, g)$ used. This transformation eventually leads to the mid-panel quadrature method and $1-M_{R}$ is inevitably introduced to the integrand denominator. If the rotor rotates supersonically, there is a possibility that $1-M_{R} \rightarrow 0$ though it depends on the relative position between the source and observer. An alternative frame transformation is proposed to solve this problem. Recall Eq. 4.8

$$
d \boldsymbol{y}=\frac{c d \Gamma d f d g}{\sin \vartheta}
$$

where $\vartheta$ is the angle between the radiation vector $\hat{\mathbf{r}}$ and surface normal vector $\hat{\mathbf{n}}$, and $\Gamma$ is the intersection curve (shown in Figure 4.1) between the surface $f(\boldsymbol{x}, t)=0$ and the sphere $g=\tau-t+r / c=0$, of which the latter can be interpreted as the equation of a sphere with a radius $r=c(t-\tau)$ whose centre is at the observer location $\boldsymbol{x}$ for a particular emission time $\tau$. If $\tau \rightarrow t$, then the radius of this sphere reduces to zero, i.e. collapses.

At an arbitrary emission time $\tau$, the intersecting curve between the two surfaces is the collective set of all points emitting signals that can reach the observer at the same time $t$. Eq. 4.18 and 4.22 now becomes

$$
\begin{aligned}
4 \pi p_{T}^{\prime}(\boldsymbol{x}, t)= & \frac{\partial}{\partial t} \int_{-\infty}^{t} \int_{f=0, g=0} \frac{c Q_{j} n_{j}}{r^{*} \sin \vartheta} d \tau d \Gamma-M_{0} \frac{\partial}{\partial t} \int_{-\infty}^{t} \int_{f=0, g=0} \frac{c \tilde{r}_{3} Q_{j} n_{j}}{r^{*} \sin \vartheta} d \tau d \Gamma \\
& -U_{0} \int_{-\infty}^{t} \int_{f=0, g=0} \frac{c \tilde{r}_{3}^{*} Q_{j} n_{j}}{r^{* 2} \sin \vartheta} d \tau d \Gamma \\
4 \pi p_{L}^{\prime}(\boldsymbol{x}, t)= & \frac{\partial}{\partial t} \int_{-\infty}^{t} \int_{f=0, g=0} \frac{L_{i j} n_{j} \tilde{r}_{i}}{r^{*} \sin \vartheta} d \tau d \Gamma+\int_{-\infty}^{t} \int_{f=0, g=0} \frac{c L_{i j} n_{j} \tilde{r}_{i}^{*}}{r^{* 2} \sin \vartheta} d \tau d \Gamma
\end{aligned}
$$




\section{AEROACOUSTIC SOLVER FOR ROTOR-INDUCED NOISE}

This algorithm eliminates the Doppler singularity but it does have a singularity in the integrand if the surface normal vector $\hat{\mathbf{n}}$ is parallel to the radiation vector $\hat{\mathbf{r}}$, i.e. $\sin \vartheta=0$. Previous studies (Brentner \& Farassat [2003]) have shown that this algorithm is very computationally demanding for the construction of the intersection curve $\Gamma$. It must be done a number of times for every single time step.

\subsubsection{Emission surface algorithms}

The third way of frame transformation given by Brentner \& Farassat [2003] is to convert $\left(y_{3}, \tau\right)$ into $(F, g)$. Recall Eq. 4.9,

$$
d \boldsymbol{y} d \tau=\frac{d \Sigma d F d g}{\|\nabla F\|}
$$

where $F(\boldsymbol{y} ; \boldsymbol{x}, t)=f(\boldsymbol{y}, t-r / c)$, and $\hat{N}_{3}$ is the third component of the unit normal vector to the $\Sigma$ surface $F=0$, which is the emission surface containing all the points that emit signals reaching the observer at a same reception time. Introducing $\Lambda=\|\nabla F\|$,

$$
\begin{aligned}
\Lambda & =\|\nabla f\|=\left\|\frac{\partial f}{\partial y_{i}}-\frac{1}{c} \frac{\partial f}{\partial t} \frac{\partial r}{\partial y_{i}}\right\| \\
& =\left\|\hat{\mathbf{n}}-\frac{v_{n}}{c} \hat{\mathbf{r}}\right\| \\
& =\sqrt{1-2 M_{n} \cos \vartheta+M_{n}^{2}},
\end{aligned}
$$

where $\partial f / \partial t=-v_{n}, \partial r / \partial y_{i}=-\hat{\mathbf{r}}$, and $\vartheta$ is the angle between the unit normal vector $\hat{\mathbf{n}}$ and radiative vector $\hat{\mathbf{r}}$, and $M_{n}=M_{i} n_{i}$. Thus, Eq. 4.18 and 4.22 become

$$
\begin{aligned}
4 \pi p_{T}^{\prime}(\boldsymbol{x}, t)= & \frac{\partial}{\partial t} \int_{F=0}\left[\frac{Q_{j} n_{j}}{r^{*} \Lambda}\right]_{\tau} d \Sigma-M_{0} \frac{\partial}{\partial t} \int_{F=0}\left[\frac{\tilde{r}_{3} Q_{j} n_{j}}{r^{*} \Lambda}\right]_{\tau} d \Sigma \\
& -U_{0} \int_{F=0}\left[\frac{\tilde{r}_{3}^{*} Q_{j} n_{j}}{r^{* 2} \Lambda}\right]_{\tau} d \Sigma \\
4 \pi p_{L}^{\prime}(\boldsymbol{x}, t)= & \frac{\partial}{\partial t} \int_{F=0}\left[\frac{L_{i j} n_{j} \tilde{r}_{i}}{r^{*} \Lambda}\right]_{\tau} d \Sigma+\int_{F=0}\left[\frac{L_{i j} n_{j} \tilde{r}_{i}^{*}}{r^{* 2} \Lambda}\right]_{\tau} d \Sigma .
\end{aligned}
$$




\section{AEROACOUSTIC SOLVER FOR ROTOR-INDUCED NOISE}

The emission surface formulation is perhaps so far the most accurate method found. Not only does Doppler singularity have no effects on it, the integrand in Eq. 4.15 can also be integrated even if when $\Sigma=0$ (see Farassat \& Myers [1988] for details). However, the construction of this emission surface is very complicated, and to the best knowledge of the author, only Brentner \& Farassat [2003] and Ianniello [2007] successfully implemented the emission surface algorithm. In order to construct this emission surface, Brentner [1994] devised an approach labelled as Marching Cubes Algorithm, which is based on a computer graphics method to produce iso-surfaces. Similar to the collapsing sphere algorithm, this marching cubes algorithm is very computationally demanding. For the same purpose, Ianniello [1999] proposed a even more sophisticated K-algorithm and improved it recently [2007]. However, understanding this method requires very advanced mathematical knowledge such as non-linear differential geometry, and numerical implementation is also of great complexities. From the literature survey, it has not been found that the K-algorithm has been used by anybody else except by Ianniello for aerodynamic noise prediction of rotor blades, which perhaps is mainly due to its complexities in both emission surface construction and numerical implementation.

\subsubsection{Permeable surface integration scheme}

In the formulations and algorithms that have been explained above the quadrupole noise is omitted and it is legitimate to do so in the subsonic cases. However, extensive studies have been done to show that quadrupole source can contribute more than enough to the total sound field so it cannot be neglected if the rotor blade speed is near transonic/supersonic. The direct evaluation of this nonlinear volume integral requires a 4-dimensional integration and double differentiation, which is of great mathematical complexities and large computational cost.

di Francescantonio [1997] proposed a different perspective to study this problem. The FW-H equation (Eq. 3.27) shows that the any physical acoustic sources enclosed by $f=0$ only contribute through the surface source terms, i.e. thickness and loading noise, and any physical sources of sound effects outside $f=0$ contribute through the volume source terms. Hence, if all the physical sources are 


\section{AEROACOUSTIC SOLVER FOR ROTOR-INDUCED NOISE}

inside the surface $f=0$ then there is no contribution from the volume source, i.e., the quadrupole source can be neglected. It was suggested that a fictitious surface can be used as the integral surface instead of using the rotor blade surface. Without the quadrupole volume source, the numerical evaluation is much easier and less computational demanding. It is also worth mentioning that Ffowcs-Williams \& Hawkings [1969] understood the significance of using this permeable surface technique in the paper where they proposed the original FW-H equation. However, this method requires very accurate near-field flow description from CFD. Not until recently has CFD become matured enough to provide fine enough flow data to enable the implementation of $\mathrm{FW}-\mathrm{H}$ on a permeable surface.

In the derivation of Farassat Formulation 1A the integral surface is chosen to be the rotor blade surface, the integral representations of thickness and loading noise are

$$
\begin{aligned}
4 \pi p_{T}^{\prime}(\boldsymbol{x}, t)= & \int_{S_{b}}\left[\frac{\rho_{0}\left(\dot{v}_{n}+v_{\dot{n}}\right)}{r\left(1-M_{r}\right)^{2}}\right]_{\tau} d S_{b} \\
& +\int_{S_{b}}\left[\frac{\rho_{0} v_{n}\left(\mathbf{r} \dot{\mathbf{M}}+c M_{r}-c M^{2}\right)}{r^{2}\left(1-M_{r}\right)^{3}}\right]_{\tau} d S_{b}, \\
4 \pi p_{L}^{\prime}(\boldsymbol{x}, t)= & \frac{1}{c} \int_{S_{b}}\left[\frac{i_{r}}{r\left(1-M_{r}\right)^{2}}\right]_{\tau} d S_{b} \\
& +\int_{S_{b}}\left[\frac{l_{r}-l_{M}}{r^{2}\left(1-M_{r}\right)^{2}}\right]_{\tau} d S_{b} \\
& +\frac{1}{c} \int_{S_{b}}\left[\frac{l_{r}\left(\mathbf{r} \dot{\mathbf{M}}+c M_{r}-c M^{2}\right)}{r^{2}\left(1-M_{r}\right)^{3}}\right]_{\tau} d S_{b},
\end{aligned}
$$

in which $S_{b}$ denotes the blade surface, $M_{r}, \mathbf{n}, \mathbf{r}, u, v$ carry the same meaning as previous defined but absence of convective flow, and $l_{i}=\left(p-p_{0}\right) n_{i}$. The 


\section{AEROACOUSTIC SOLVER FOR ROTOR-INDUCED NOISE}

permeable integral representation can be written in a similar form

$$
\begin{aligned}
4 \pi p_{T}^{\prime}(\boldsymbol{x}, t)= & \int_{S_{p}}\left[\frac{\rho_{0}\left(\dot{U}_{n}+U_{\dot{n}}\right)}{r\left(1-M_{r}\right)^{2}}\right]_{\tau} d S_{p} \\
& +\int_{S_{p}}\left[\frac{\rho_{0} U_{n}\left(\mathbf{r} \dot{\mathbf{M}}+c M_{r}-c M^{2}\right)}{r^{2}\left(1-M_{r}\right)^{3}}\right]_{\tau} d S_{p}, \\
4 \pi p_{L}^{\prime}(\boldsymbol{x}, t)= & \frac{1}{c} \int_{S_{p}}\left[\frac{\dot{L}_{r}}{r\left(1-M_{r}\right)^{2}}\right]_{\tau} d S_{p} \\
& +\int_{S_{p}}\left[\frac{L_{r}-L_{M}}{r^{2}\left(1-M_{r}\right)^{2}}\right]_{\tau} d S_{p} \\
& +\frac{1}{c} \int_{S_{p}}\left[\frac{L_{r}\left(\mathbf{r} \dot{\mathbf{M}}+c M_{r}-c M^{2}\right)}{r^{2}\left(1-M_{r}\right)^{3}}\right]_{\tau} d S_{p},
\end{aligned}
$$

in which $S_{p}$ denotes the permeable surface and

$$
\begin{aligned}
U_{i} & =v_{i}+\frac{\rho}{\rho_{0}}\left(u_{i}-v_{i}\right), \\
L_{i} & =\left(p-p_{0}\right) n_{i}+\rho u_{i}\left(u_{n}-v_{n}\right)
\end{aligned}
$$

where it can be seen that if $f=0$ is the rotor blade surface, $u_{i}=v_{i}, U_{i}=v_{i}$, the permeable formulations are reduced to the Farassat Formulation 1A.

The advantage of Formulation $1 \mathrm{C}$ is that the integral surface $f=0$ was not chosen to be the rotor blade surface at all. Hence, the integral formulations of thickness and loading noise, Eq. 4.20 and Eq. 4.23 are in fact the permeable integral formulations already and no further re-expressing is needed. If the mean flow has no velocity, Eq. 4.20 and Eq. 4.23 can be simplified to Eq. 3.42 and 3.43 .

\subsection{Summary}

In this chapter, thorough mathematical formulations for solving the convective FW-H equation have been provided. A particular focus was dedicated to deriving the integral solutions which can be numerically implemented for rotor-induced noise predictions. Owing to the sub/transonic rotor tip speeds, impermeable and permeable integral representations were both given in terms of thickness and 


\section{AEROACOUSTIC SOLVER FOR ROTOR-INDUCED NOISE}

loading noise integral equations.

Both observer-time and source-time dominant algorithm are discussed in some detail for different regimes. The former one is very efficient and robust for the subsonic conditions, however; it can be very inefficient for the supersonic conditions, and in that case, the source-time dominant algorithm should be adopted though additional efforts will be required for interpolating the chaotic pressuretime signatures into a ordered time sequence evenly spaced, such as the natural cubic spline interpolation(Cheney \& Kincaid [2008]).

The Doppler singularity in the integral representations has been carefully analysed. In the presence of supersonic flow conditions, this may cause serious problems for numerical computations. The collapsing sphere and emission surface algorithm can be employed to eliminate the singularity, however; numerical programming will be further complicated. The permeable surface integral approach can be used as an alternative, for which high-quality CFD simulations are required to provide flow quantities as inputs. Also, a careful selection of observer location may help to remove the presence of singularity as the Doppler factor $1-M_{r}$ depends on the relative positions between source and observer. Strictly speaking, the singularity can only be present if the radiation Mach number $M_{r}$ is exactly equal to 1 , which is possible to avoid by a careful selection of observer positions.

The next chapter will present some numerical results of rotor-induced noise using the integral equations and temporal algorithms developed in this chapter. 
"Experimental confirmation of a prediction is merely a measurement. An experiment disproving a prediction is discovery."

Enrio Fermi (1901 - 1954)

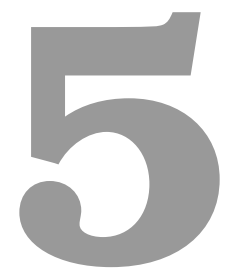

\section{Applications and validations of the developed aeroacoustic solver}

This chapter presents the numerical evaluations of aerodynamic noise due to two different rotor confirgurations. Experimental measurements have been used to validate the computational results, where good agreement is obtained.

In the present study, two cases are used. The first one is an extensively studied helicopter rotor, namely, NACA 0012 UH1H helicopter rotor, which has a uniform cross-section. The second one is the SRV2 centrifugal compressor that has been experimentally studied at DLR (German Aerospace Centre). Adequate experimental data have been obtained for validation purpose.

The SRV2 impeller is a well-known transonic centrifugal compressor that was designed by Krain at DLR. It has received great attention over the last 20 years for its flow field details, aerodynamic performances, and its redesigns. 


\section{APPLICATIONS AND VALIDATIONS OF THE DEVELOPED AEROACOUSTIC SOLVER}

\subsection{Coding algorithm development}

The general methodology is to conduct the CFD analysis first to get the near-field fluid quantities around the blades, which was completed using ANSYS CFX software. The RANS (Reynolds Averaged Navier Stokes) equation solver is used first, which has the advantage that the temporal derivatives of the fluid quantities, such as, $\dot{\rho}, \dot{p}, \dot{\boldsymbol{u}}_{i}$ on the integral surface are all zero owing to the time-independence feature of RANS and thus, save the computational cost. However, there is a tradeoff for the accuracy. The URNAS (Unsteady Reynolds Averaged Navier Stokes) can then be adopted in order to get the higher resolution results. The central difference method is used for the numerical differentiations of the aforementioned temporal derivatives. Three algorithms have been constructed for numerical implementations of FW-H solver. Figure. 5.1 shows a retarded-time algorithm for subsonic conditions, which has been used for the NACA 0012 rotor configuration.

Codes were written in FORTRAN 90 to perform the Farassat's Formulations 1A in both impermeable and permeable conditions using retarded-time algorithm. The mid-panel quadrature method was adopted for numerical integrations, thus it was assumed that the fluid quantities on each panel is the same everywhere as those on the centre of panel. The coordinates $\boldsymbol{y}_{i}$, unit normal vector components $\boldsymbol{n}_{i}$, panel surface area $\delta s_{i}$, fluid velocity components $\boldsymbol{u}_{i}$, pressure $p_{i}$, density $\rho_{i}$, rotational speed $\omega$ are all obtained from CFX simulation results and used as inputs into the program. The observer coordinates $\boldsymbol{x}$ can be specified by any code user. The total observer time $t$ can be set to one revolution period or more and discretised into $N_{t}$ steps. The greater the value of $N_{t}$ is, the higher resolution of acoustic signature figure and the larger computational cost will be. In the present study $N_{t}$ was set to 256 for one revolution, giving rise to both good resolution and acceptable cost. The integral surface is divided into $N_{p}$ panels in CFX. At a certain a given observer time $t_{j}$, the retarded times $\tau_{i j}$ of every panel $\boldsymbol{y}_{i}$ are calculated by Eq. 4.26 using Newton-Raphson method. The acoustic pressure $p_{i j}^{\prime}$ will be iteratively calculated until $i$ and $j$ reach their maximum values, $N_{t}$ and $N_{p}$. In the end, the pressure contributions from all the panels at every observer time step will be summed, i.e. $p_{s}^{\prime}\left(t_{j}\right)=\sum_{i=1}^{N_{p}} p_{i j}^{\prime}$.

The other algorithm (Figure 5.2) is applied to the compressor rotor noise 
prediction using the advanced time method with steady CFD results. As this algorithm is source-time dominant, the emission time is required to be first discretised evenly and the reception time sequences are calculated using Eq. 4.33. It should be noted that the interval of the computed reception time sequences will not be constant due to nonlinearity of the governing equation, and thus, the pressure time signatures need to be interpolated into a given time sequences with constant interval in order to perform the sum of all pressure contributions. In order to study the compressor noise characteristics, the pressure-time signature must be converted into the sound pressure level (SPL)-frequency domain, for which the Fast Fourier Transform (FFT) analysis can be used.(Brigham [1974])

$$
S P L=20 \log _{10}\left(\frac{p^{\prime}}{p_{\text {ref }}}\right)
$$

in which, $p_{\text {ref }}=20 \mu p a$. 


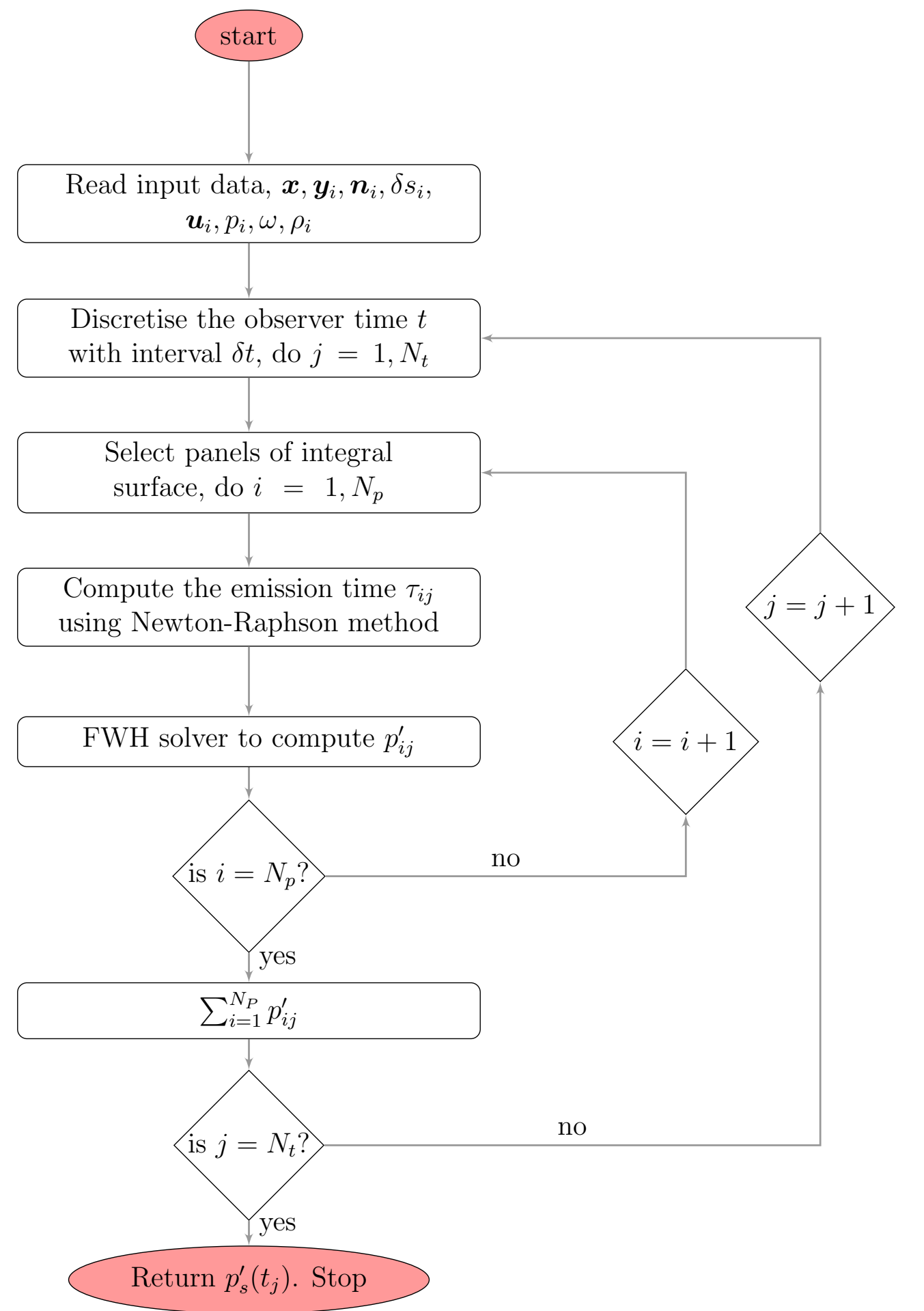

Figure 5.1: Flow chart of FW-H solver programming: a retarded -time algorithm 


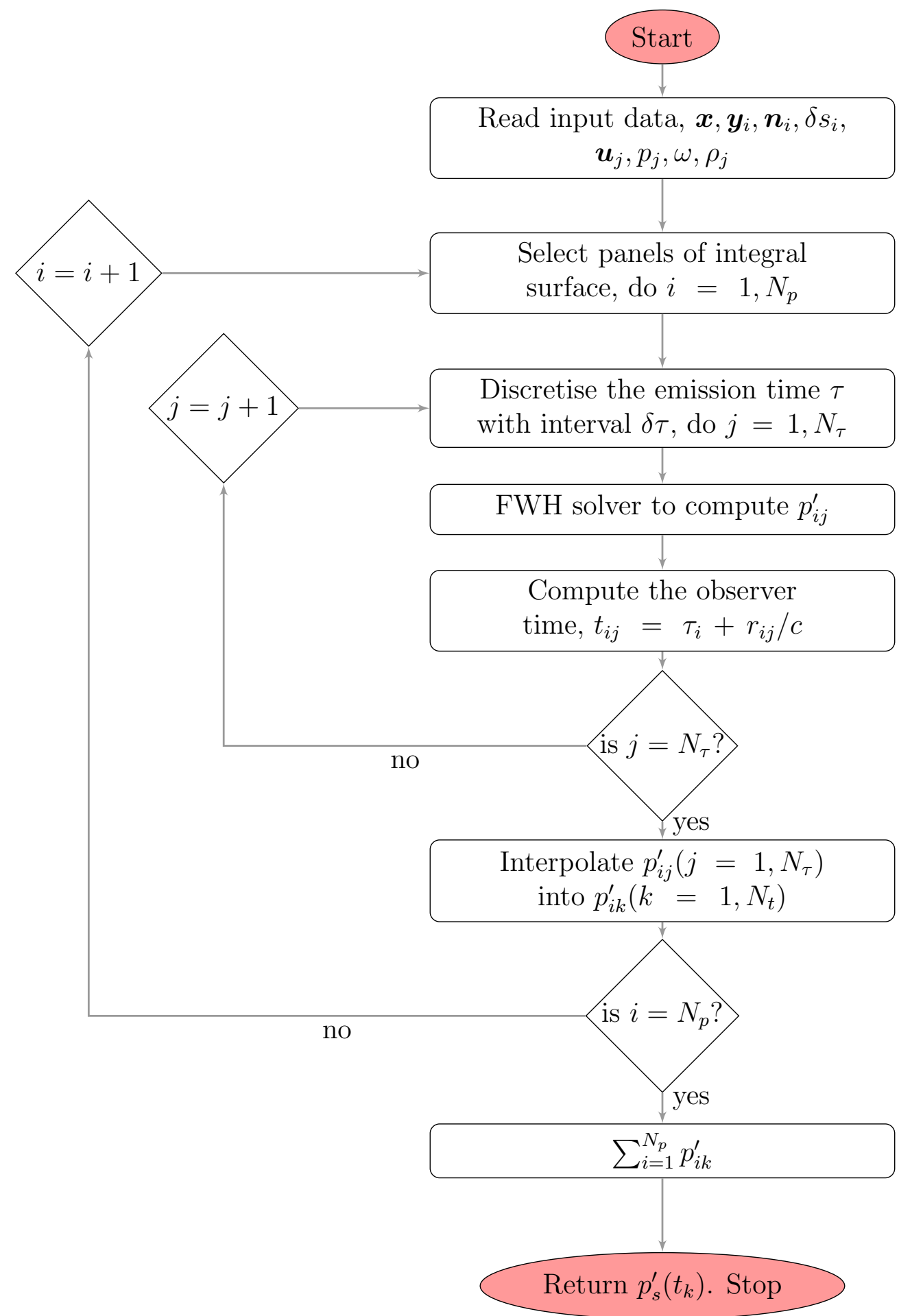

Figure 5.2: Flow chart of FW-H solver programming: an advanced -time algorithm with RANS 


\subsection{UH1H NACA 0012 helicopter rotor}

\subsubsection{Geometry definition}

The rotor used in the test is a $1 / 7^{\text {th }}$ scale model of a $\mathrm{UH} 1 \mathrm{H}$ main rotor with straight untwisted blades and has a NACA 0012 airfoil cross-section. The rotor has a span length $1.045 \mathrm{~m}$ and chord length $0.0762 \mathrm{~m}$, giving a aspect ratio of 13.71(Ladson et al. [1996]). The uniform cross section is described by the equation

$$
\begin{aligned}
\frac{z}{l_{c}}= \pm 0.6\{ & 0.2969\left(\frac{x}{l_{c}}\right)^{1 / 2}-0.126\left(\frac{x}{l_{c}}\right)-0.3537\left(\frac{x}{l_{c}}\right)^{2} \\
& \left.+0.2843\left(\frac{x}{l_{c}}\right)^{3}-0.1015\left(\frac{x}{l_{c}}\right)^{4}\right\}
\end{aligned}
$$

where $l_{c}$ is the chord length. The cross-section view of the rotor is shown in Figure. 5.3.

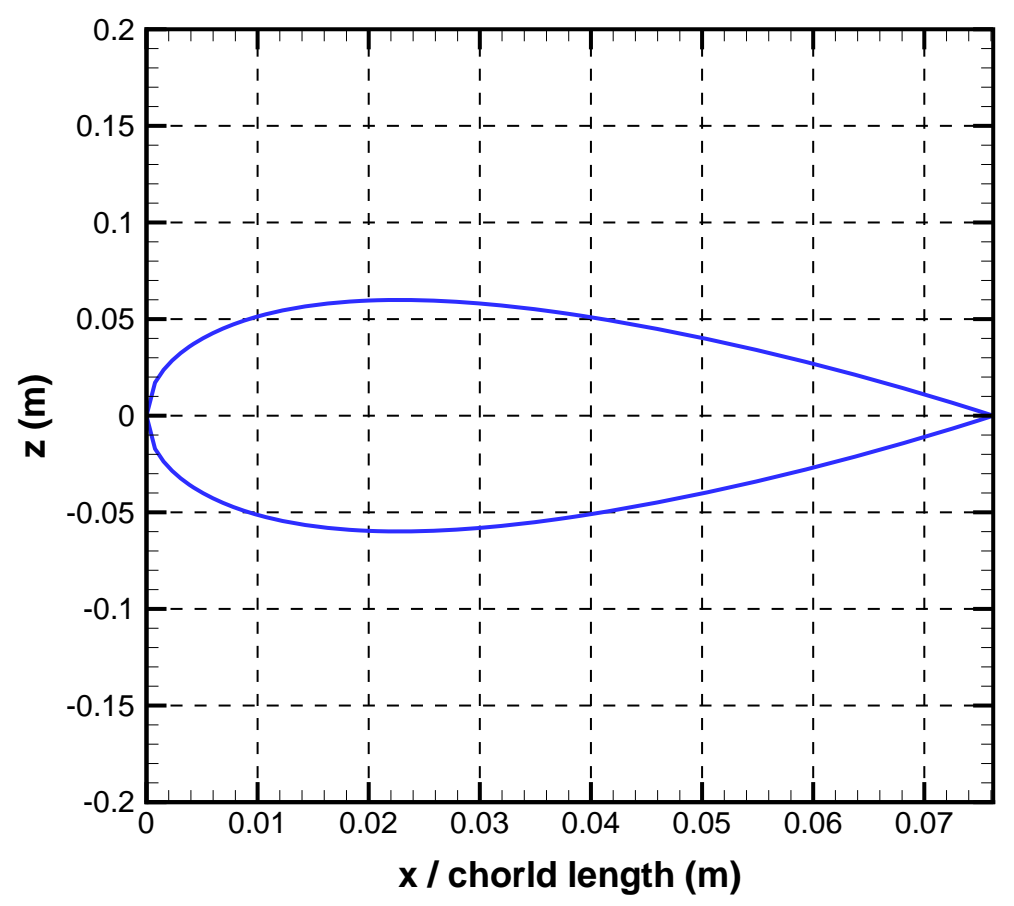

Figure 5.3: Cross-section of NACA 0012 rotor 


\section{APPLICATIONS AND VALIDATIONS OF THE DEVELOPED}

AEROACOUSTIC SOLVER

\subsubsection{Experimental set-up}

The helicopter rotor rotates under constant speeds in hovering, and a microphone used to record the pressure fluctuations is set to be in-pane with blades and fixed at a distance 3.09 radii. Figure. 5.4 shows the schematics of the experimental set-up of noise measurement.

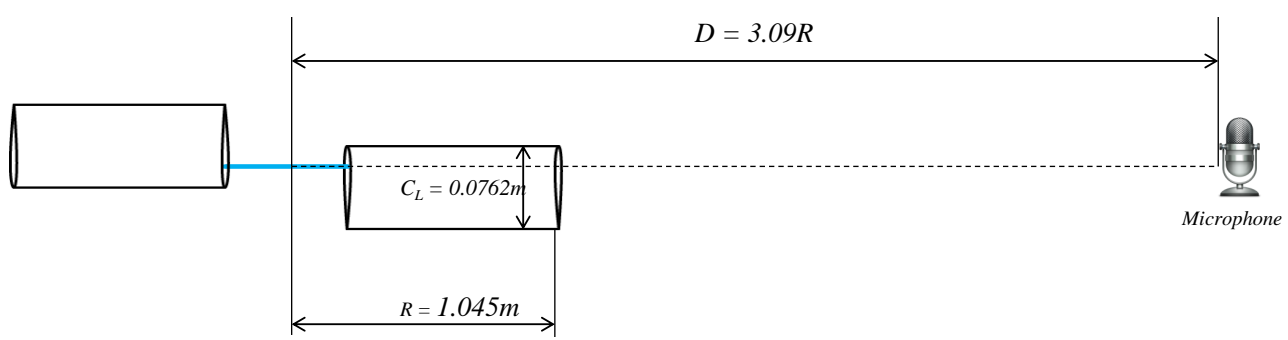

Figure 5.4: Schematics of NACA 0012 rotor noise measurement set-up

\subsection{Numerical results of UH1H NACA 0012 ro- tor noise}

In Chapter 3, different integral formulations have been developed for the numerical predictions of rotor-induced noise taking account of different blade tip Mach numbers, as shown in Table. 5.1. Farassat Formulation 1A coupling with the retarded-time method is very suitable for subsonic cases. The rotational speed of the helicopter rotor is varied to have different tip Mach numbers, ranging from 0.80 to 0.88. Experiments were performed by Purcell [1988] to measure the noise produced at these different rotor speeds. It has been identified that quadrupole source noise plays an important role in the total noise field when the tip Mach number is over 0.80 . The numerical implementation of $\mathrm{FW}-\mathrm{H}$ method requires the discretisation of the integral surfaces, either permeable or impermeable. An example is given for illustration purpose in Figure 5.5. As the NACA 0012 model has an analytical formulation for the cross-section, analytical meshing can be done on the integral surfaces. However, it is more convenient to use CFD mesh as the flow quantities are needed to be taken from the centre of of each panel. 


\section{APPLICATIONS AND VALIDATIONS OF THE DEVELOPED AEROACOUSTIC SOLVER}

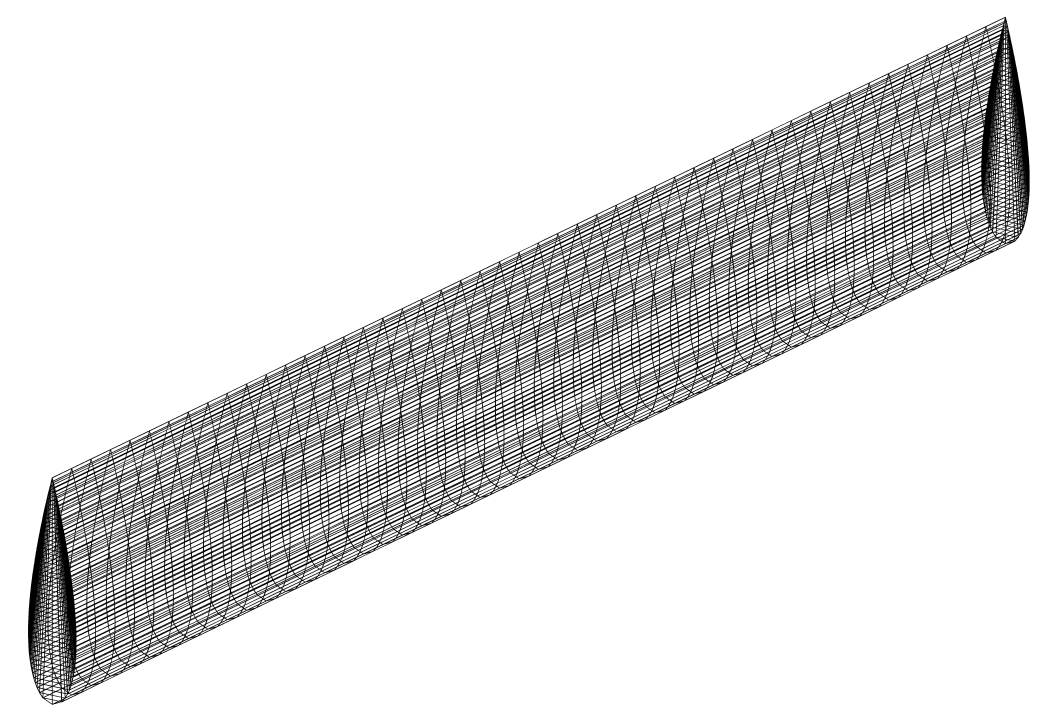

Figure 5.5: Discretized rotor blade surface

Table 5.1: Regimes of integral formulations for subsonic or transonic cases

\begin{tabular}{ll}
\hline Subsonic $\left(M_{t i p}<0.80\right)$ & Transonic/supersonic $\left(M_{t i p} \geq 0.80\right)$ \\
Impermeable integration & Permeable integration \\
Retarded/advanced time method & Retarded/advanced time method \\
\hline
\end{tabular}

Interpolations are required later if using the analytical mesh.

The blade surface was first chosen as the integral surface to calculate the thickness and loading noise using Farassat Formulation 1A coupled with the retarded-time method. In Figure 5.6 and 5.7 it can be seen that the loading noise is much less influential than the thickness noise, and that is because the observer was placed in-plane with the rotor as shown in Figure 5.4. One can find that the sum of thickness and loading noise still underestimates the acoustic pressure generated by high speed rotors by comparing with experimental results, which indicates that the quadrupole source noise contribute more than enough to be neglected. Thus, the permeable surface integration is used in order to get more accurate results. One of the difficulties is that the location of the permeable surface cannot be known a priori, and one needs to iterate until converged results emerge. It has been suggested by other researchers (Brentner \& Farassat [2003], 


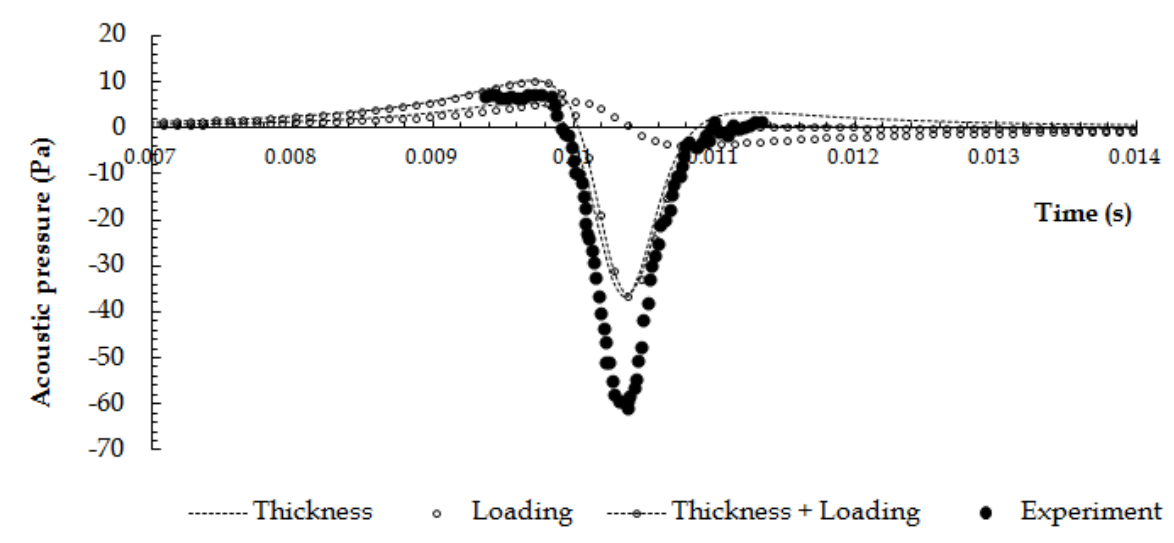

Figure 5.6: $M_{\text {tip }}=0.80$, comparison of predictions (blade usrface) and experiment

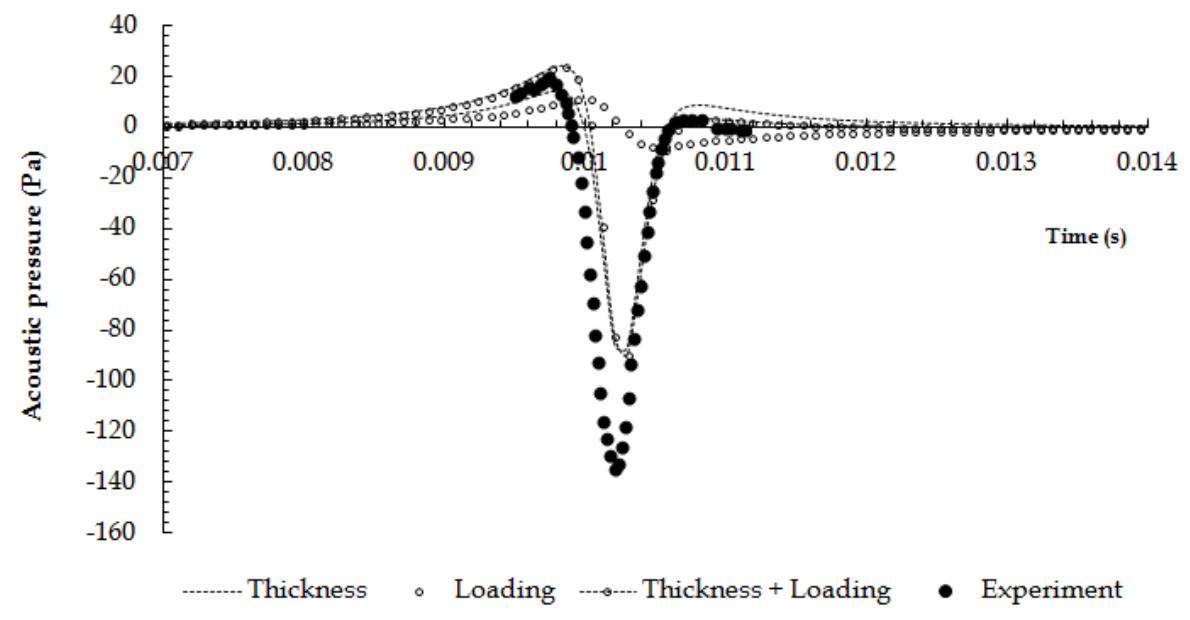

Figure 5.7: $M_{t i p}=0.85$, comparison of predictions (blade surface) and experiment 


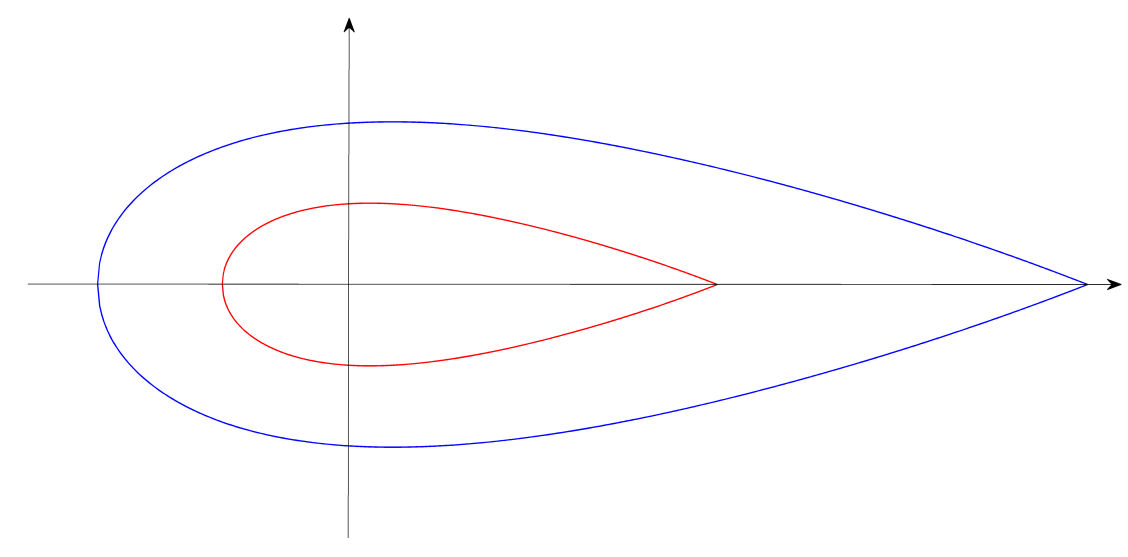

Figure 5.8: Permeable and impermeable surface cross-section

Tadamasa \& Zangeneh [2011]) that the permeable surfaces can be made as the same shape of the rotor blade with a doubled chord length (see Figure 5.8) and the span length can be varied gradually as performing the convergence study. In the present work, the span length is increased from $2 \%$ to $12 \%$ with a constant interval (examples given in Figure 5.9, the enlarged tip region in Figure 5.10). Numerical results of this rotor-induced noise are shown in Figure 5.11, 5.12 and 5.13 .

It can be observed that the results obtained from different permeable surfaces converge everywhere except at the negative peaks. As the span length is increased steadily, the negative peak also increases. It is interesting to note that for $M_{t i p}=0.80$ and $M_{t i p}=0.85$ the numerical predictions agree with experimental measurements very well, and the negative peaks are both captured. However, the numerical evaluations did not capture the negative peak for $M_{\text {tip }}=0.88$ in spite of varying the span length to the allowed maximum. This was also reported by Morgans et al. [2005]. The discrepancy is likely due to the underestimation of loading noise since the computational blade drag predictions can be much smaller than that in experiments. It was also observed by Baeder et al. [1997] and Boxwell et al. [1979] that the negative pressure peak is increased about $25 \%$ even if the tip Mach number is slightly increased to 0.89, as shown in Figure 5.14. This implies that shock delocalisations can happen if the blade tip Mach number is 


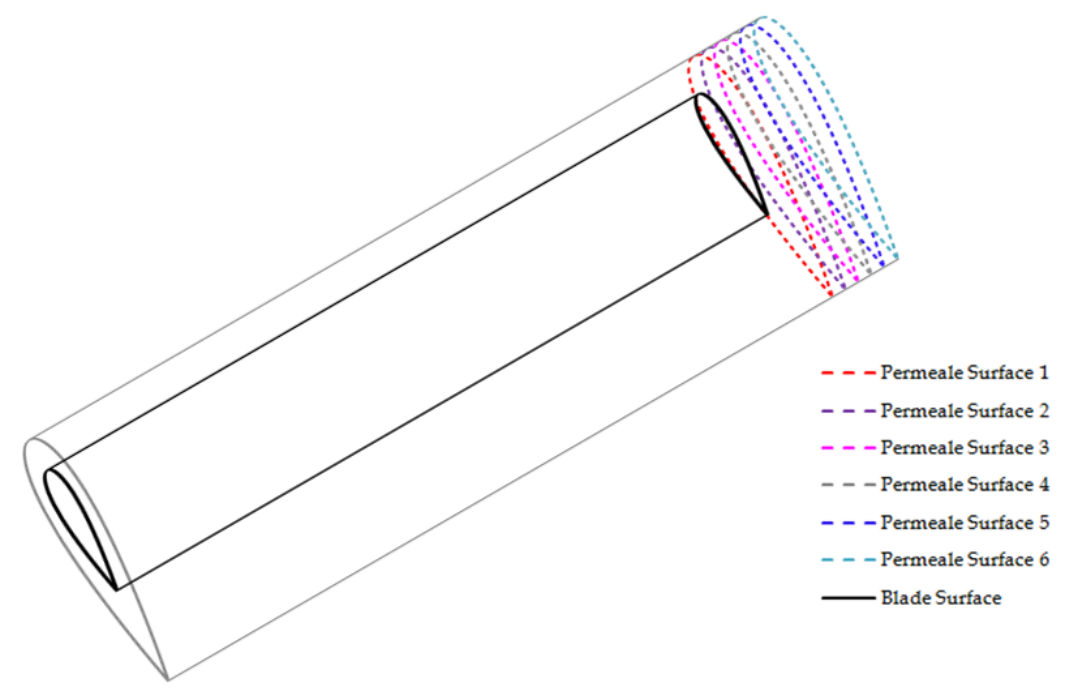

Figure 5.9: The integral data surfaces for NACA0012 rotor noise calculations. Permeable surfaces 1-6 has the doubled chordlength as the original blade, and spanlenggth increasing from $2 \%$ to $12 \%$.

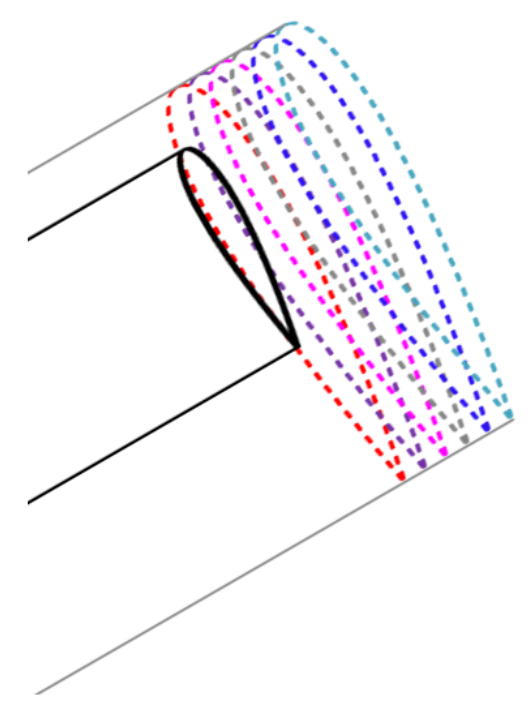

Figure 5.10: Enlarged blade tip regions of integral surfaces 


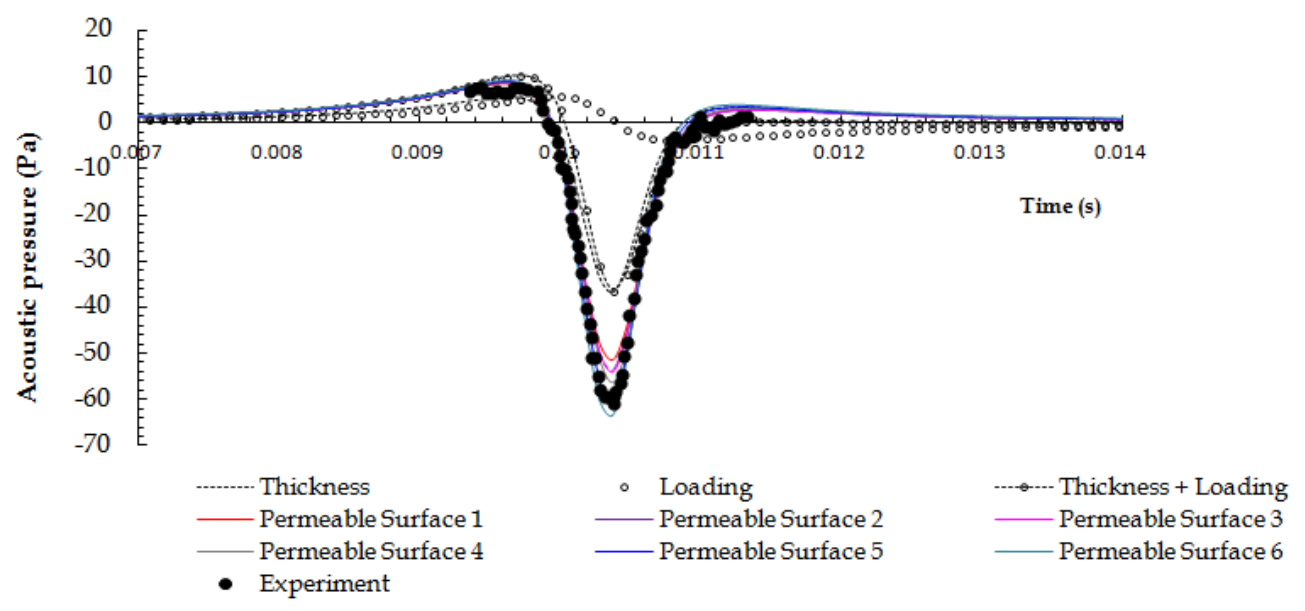

Figure 5.11: $M_{t i p}=0.80$, comparison of predictions and experiment

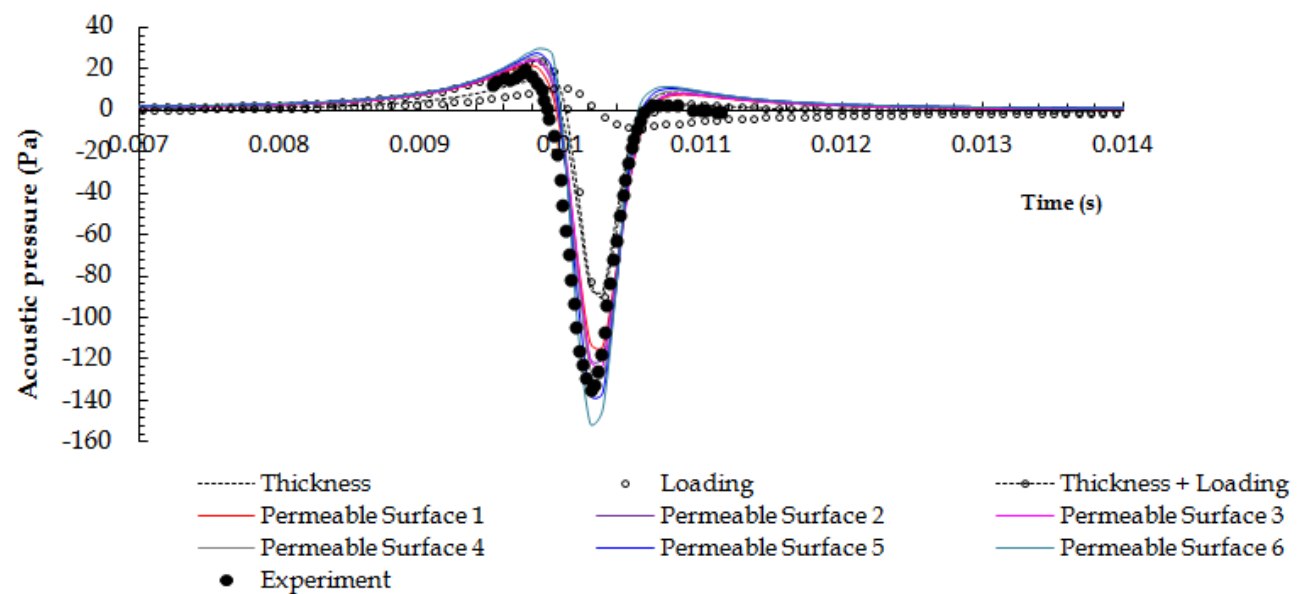

Figure 5.12: $M_{t i p}=0.85$, comparison of predictions and experiment 


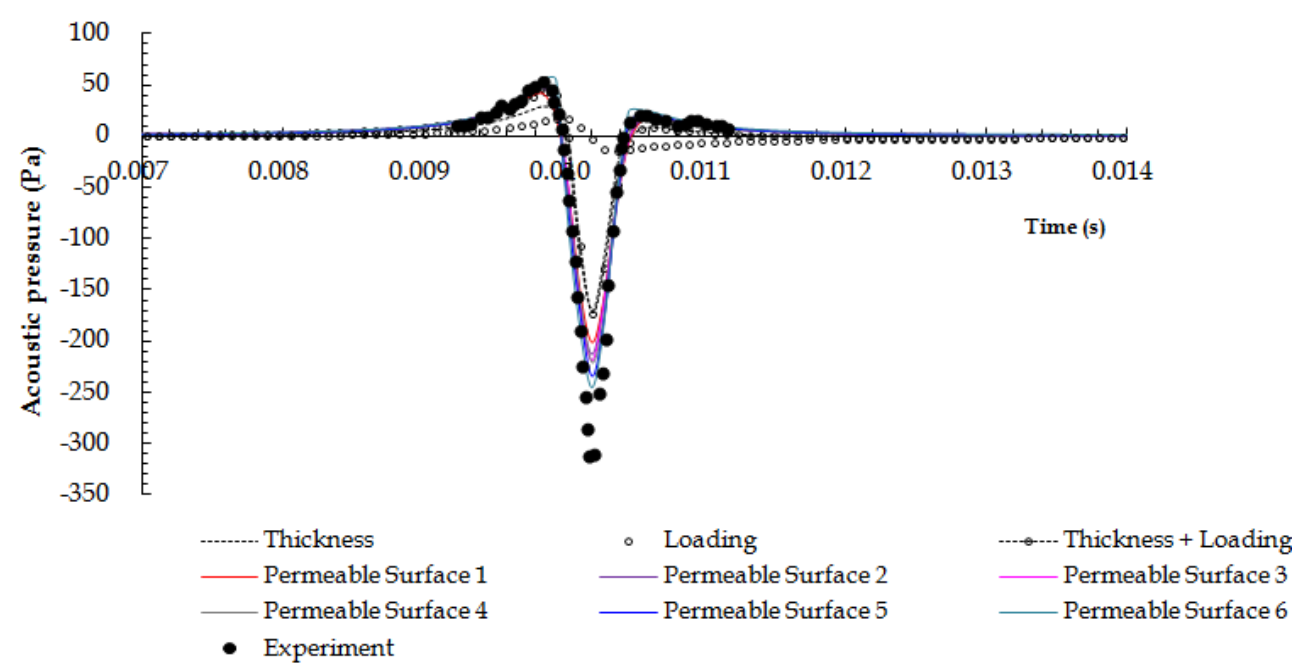

Figure 5.13: $M_{t i p}=0.88$, comparison of predictions and experiment

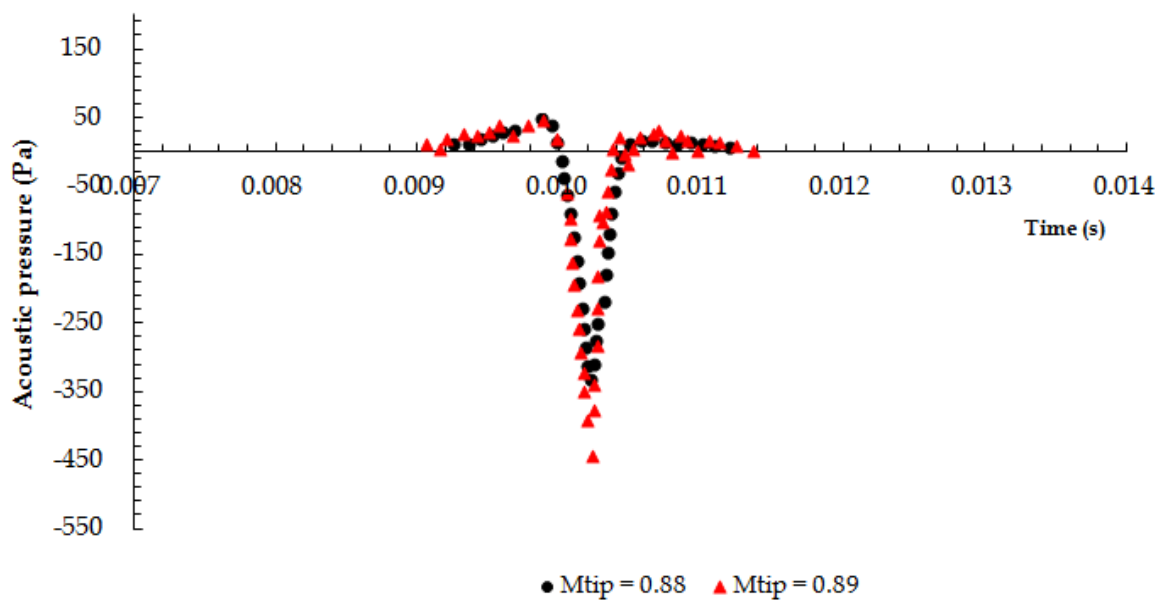

Figure 5.14: Comparisons of experimental results at different Mach numbers, $M_{\text {tip }}=0.88$, and $M_{\text {tip }}=0.89$ 


\section{APPLICATIONS AND VALIDATIONS OF THE DEVELOPED}

AEROACOUSTIC SOLVER

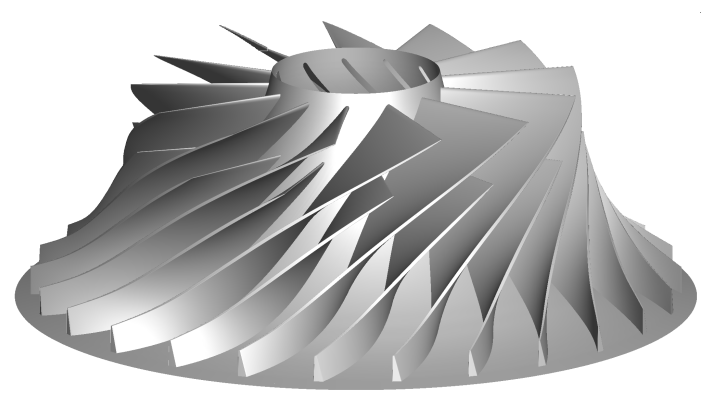

(a) Baseline SRV2 impeller

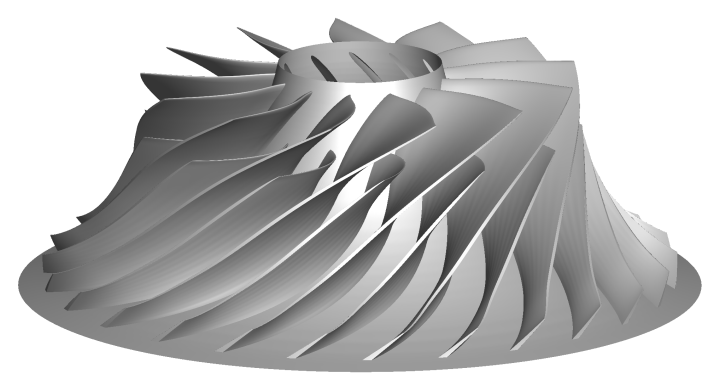

(b) Inversely redesigned SRV2 impeller

Figure 5.15: Comparisons between the baseline SRV2 impeller and its redesigned version using inverse design method

over $M_{t i p}=0.88$. This is a phenomenon that happens when the shocks near the blade tip reach the sonic cylinder as $M_{t i p}$ increases. The shocks over blade will be able to propagate to the acoustic far-field, amplifying pressure fluctuations, which cannot be predicted by steady CFD simulations.

\subsection{Transonic centrifugal compressors}

In this section we present an aeroacoustic evaluation for a well-known transonic compressor case, SRV2 impeller, which was designed and tested at DLR with extensive details published. (see Krain \& Hoffman [2007, 2008]) The experimental set-up, CFD simulations, and numerical acoustic signature under various operational speeds will all be presented. Zangeneh et al. [2011] reported a redesigned version of the SRV2 impeller using the inverse design method. The resulting impeller was found to have better performance compared to the original SRV2 impeller at all operating conditions. The detailed CFD predictions for the two impellers are used together with the acoustic solver to investigate the effect of aerodynamic changes in the inverse design impeller on its noise emissions. A comparison of two impeller geometries are given in Figs 5.15a and 5.15b, respectively. One can notice that a larger curvature appears at the LE of both redesigned main impeller and splitter blades. The redesigned blades are also more leaned at the TE. 
Table 5.2: SRV2 compressor parameters

\begin{tabular}{|l|l|}
\hline Shaft speed & $50000 \mathrm{rpm}$ \\
\hline $\begin{array}{l}\text { Flow rate at peak effi- } \\
\text { ciency for vaneless dif- } \\
\text { fuser stage }\end{array}$ & $2.73 \mathrm{~kg} / \mathrm{s}$ \\
\hline Impeller tip radius & $112 \mathrm{~mm}$ \\
\hline Tip speed & $586 \mathrm{~m} / \mathrm{s}$ \\
\hline $\begin{array}{l}\text { Blade count full/split- } \\
\text { ter }\end{array}$ & $13 / 13$ \\
\hline LE hub radius & $30 \mathrm{~mm}$ \\
\hline TE tip radius & $78 \mathrm{~mm}$ \\
\hline Blade angle LE tip & $26.5 \mathrm{deg}$ \\
\hline Blade angle TE & $52 \mathrm{deg}$ \\
\hline Exit blade height & $8.7 \mathrm{~mm}$ \\
\hline $\begin{array}{l}\text { Diffuser inclination } \\
\text { against radial }\end{array}$ & $13 \mathrm{deg}$ \\
\hline Tip clearance & $\begin{array}{l}0.5 \mathrm{~mm} \text { at inlet to } 0.3 \\
\mathrm{~mm} \text { at exit }\end{array}$ \\
\hline
\end{tabular}

\subsubsection{Test facility}

In Fig. 5.16 is shown the schematic view of the noise testing rig. The compressor is directly connected to a duct with a diameter of $157 \mathrm{~mm}$. A microphone ring with four microphones placed 90 degrees to each other is mounted on the inner wall of the duct, and this ring is placed at a distance of $165 \mathrm{~mm}$ from the leading edge of the impeller. The incoming flow is assumed to have a uniform velocity normal to the cross-section of the duct. A more detailed explanation of the experimental set-up is given in the work by Raitor \& Neise [2008].

\subsubsection{Validation}

The acoustic pressure signals received at the four measurement points were computed in time domain and then converted to the frequency domain using FFT algorithm. Figure 5.17 shows the circumferentially averaged predicted spectrum at experimental conditions. The compressor operated at the incoming flow rate $2.55 \mathrm{~kg} / \mathrm{s}$ and design speed $50000 \mathrm{rpm}$, producing a tip Mach number about 1.7. 


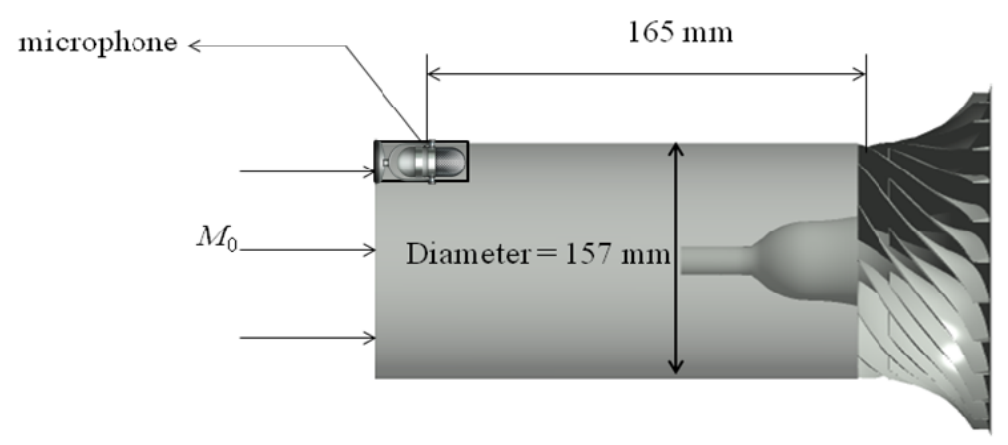

Figure 5.16: Schematics of the nosie testing rig

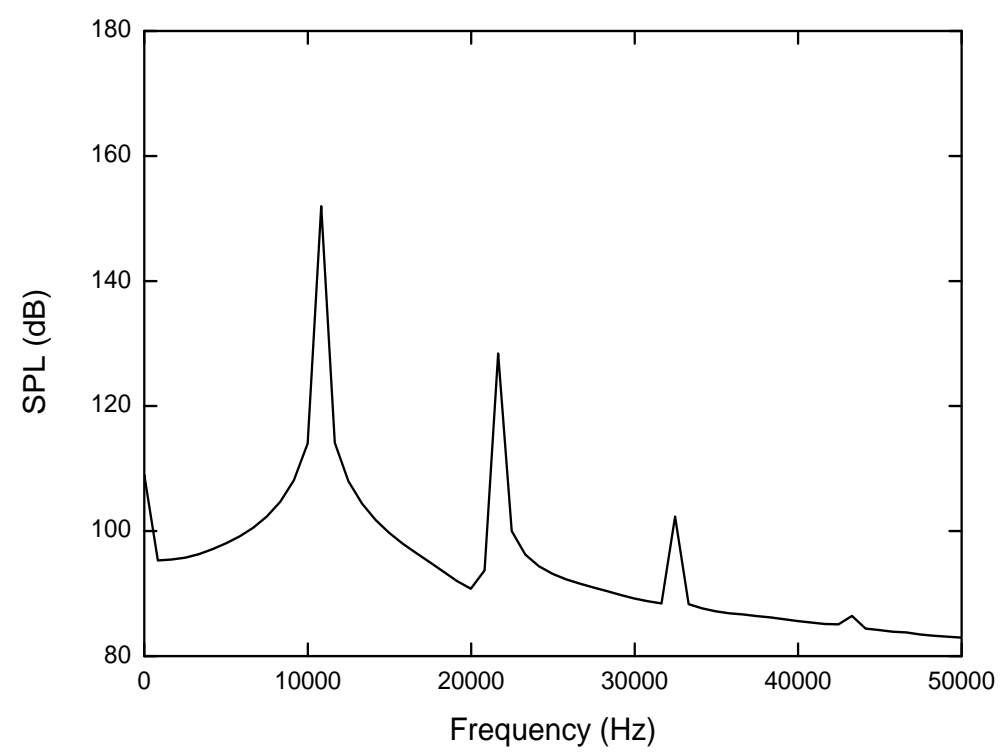

Figure 5.17: Numerical prediction of noise spectrum at $50000 \mathrm{rpm}$

The measurement spectrum is shown in Fig. 5.18. A noticeable and significant feature is that the sound level has very high magnitudes at the BPF (blade passing frequency, $10833 \mathrm{~Hz}$ ) and its harmonics, which are also clearly higher than their neighbouring components. In addition to these BPFs tones, there are also tonal components spreading over a range of harmonics of the rotational frequency, which are known as the buzz-saw noise. The dominant source of these buzz-saw components is the rotor-alone pressure of shocks attached to the supersonically rotating blade tips, and these shocks can propagate against the incoming flow to the upstream in the inlet duct.

By comparing Figs. 5.17 and 5.18 it can be seen that the tones at BPF and its 


\section{APPLICATIONS AND VALIDATIONS OF THE DEVELOPED AEROACOUSTIC SOLVER}

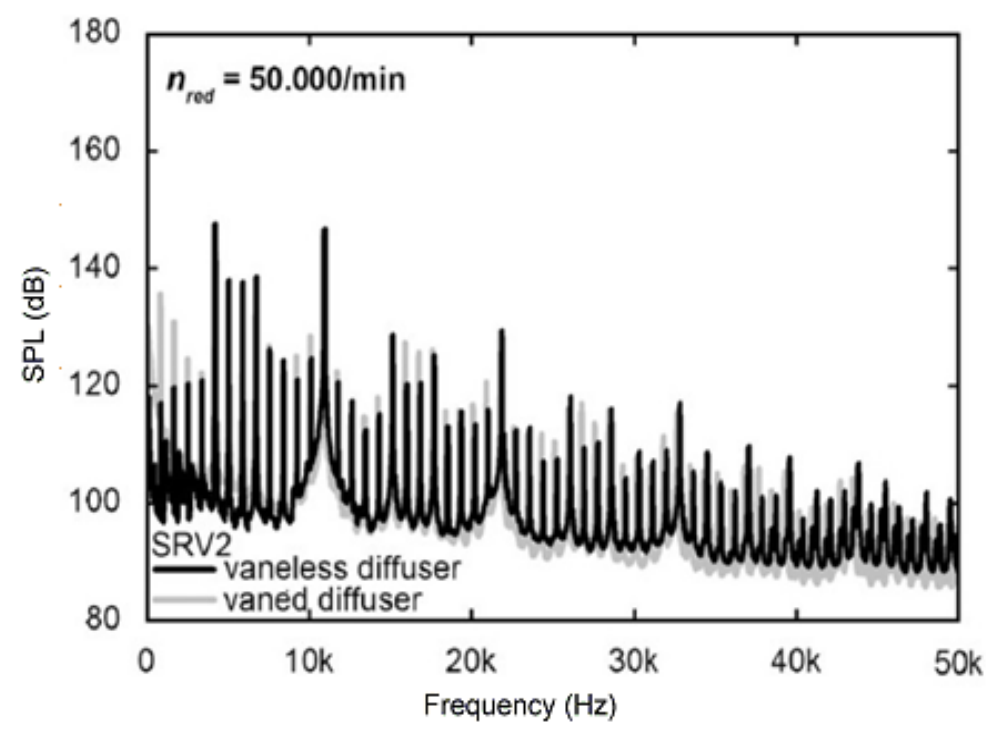

Figure 5.18: Measured noise spectrum (see Fig. 6a in Raitor \& Neise [2008])

harmonics are captured in prediction without large discrepancies, but there are no buzz-saw components on the predicted spectrum. This is because the buzz-saw tones are due to the amplitudes variations and nonlinear propagations of shocks near the blade tips, which would induce the energy redistribution of the frequency spectrum, i.e., the initial energy at BPF and its harmonics is redistributed at the harmonics of rotor shaft frequency (engine orders). A more detailed explanation of buzz-saw noise generation mechanism can be found in the work by McAlpine et al. [2006]. In a similar fan noise prediction study, Gliebe et al. [2000] reported that steady RANS simulations using multiple blade passages with perturbed (not cyclically symmetric) blade geometries are required to obtain buzz-saw tones. In this study, we only modelled one blade passage using steady-state CFD solver and assumed the perfect circumferential periodicity of flow quantities in all other 12 passages. Thus, the strength variations of the shocks and their nonlinear propagations were not included, resulting in failing to capture the buzz-saw tones at the multiples of shaft frequency.

Another important feature that deserves particular attention is that the measured spectrum has a "local maximum" before the BPF, which cannot be seen in the predicted spectrum. If one takes a closer scrutiny on the measured spectrum, 

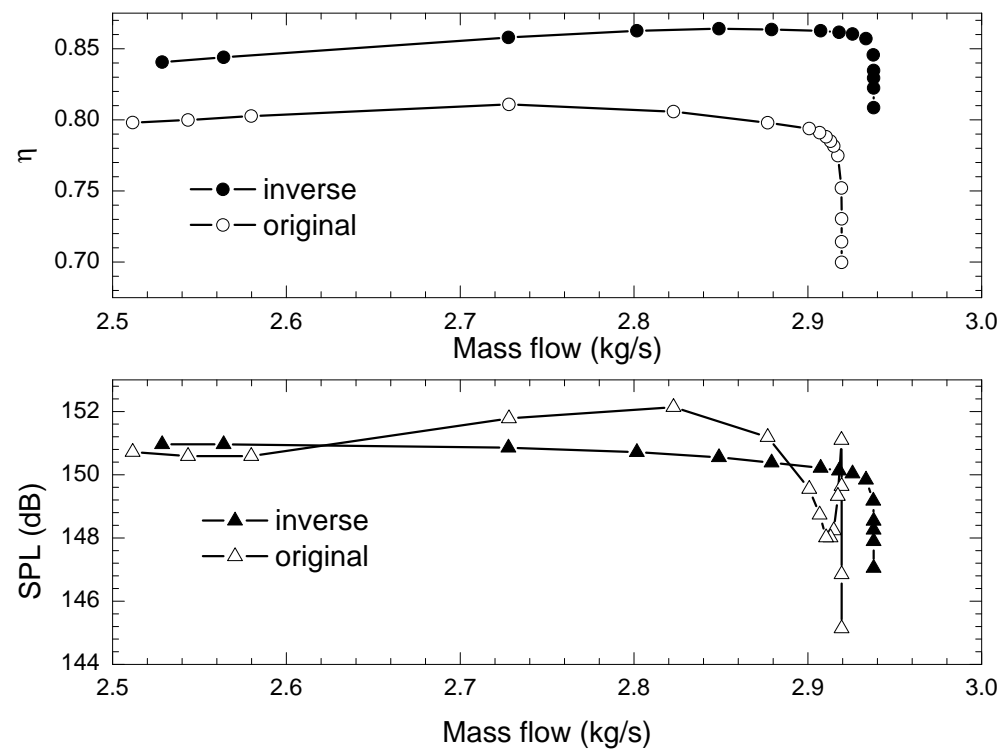

Figure 5.19: Aerodynamic and aeroacoustic performance comparisons between the original and redesigned compressor

one may find that this local maximum occurs at the $5 f_{n}\left(f_{n}\right.$ is shaft rotational frequency), which is the fifth engine order and very close to the cut-on frequency of the duct. This phenomenon is likely due to the resonance between the fifth engine order and cut-on frequency of the duct. For this study, the duct wall is not included in the numerical implementation for the simplification purpose, and this explains why this "local maximum" phenomenon did not appear on the predicted spectrum. The purpose of using a free-space space aeroacoustic model is to ascertain if design trends are favourably reducing noise emissions as intended rather than get very accurate predictions of noise field.

The sound generated by a compressor that operates at different inlet flow conditions is distinct. Therefore, it is necessary to compare the radiated noise levels at the entire operating range so as to get a comprehensive understanding of aeroacoustic behaviours. Figure 5.19 compares the aerodynamic and aeroacoustic performances of the original and redesigned compressor working from surging to choking conditions under full speed. The SPLs are the tonal magnitudes at the BPF. It is interesting to note that the sound level of the redesigned compressor has slight variations at the entire operating ranges, while the original compressor produces sound with significant changes as the mass flow varies. More impor- 

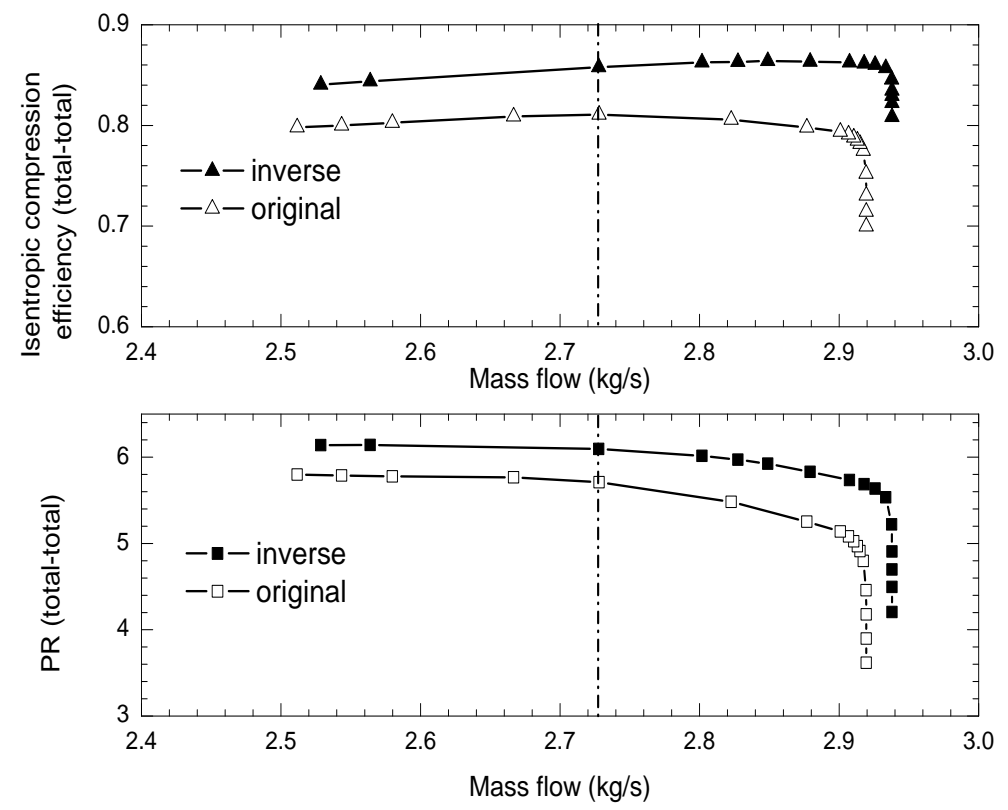

Figure 5.20: Comparison of the characteristics of the original and redesign compressor

tantly, it can be seen that sound levels generated by the redesigned are lower than those by the original when the working mass flow rate is in range from 2.72 to $2.88 \mathrm{~kg} / \mathrm{s}$. In fact, the computational work presented by Zangeneh et al. [2011] confirmed that the peak efficiency with the vaneless stage occurs around 2.73 $\mathrm{kg} / \mathrm{s}$. Thus, the redesign compressor shows lower noise emission around its peak efficiency point. However, the baseline impeller has lower level of noise at lower mass flow rates and close to the stall point.

In the following subsection, the CFD predictions of the flow physics in the blade channel at close to the peak efficiency point will be presented so as to explain the reason why the inversely redesigned compressor has a better aeroacoustic performance.

\subsubsection{CFD analysis}

The flow in the original SRV2 impeller was analyzed by using commercial CFD CFX11 by Zangeneh et al. [2011]. For this purpose a mesh independence study was carried out and a mesh size of $880 \mathrm{k}$ nodes was eventually used for each 

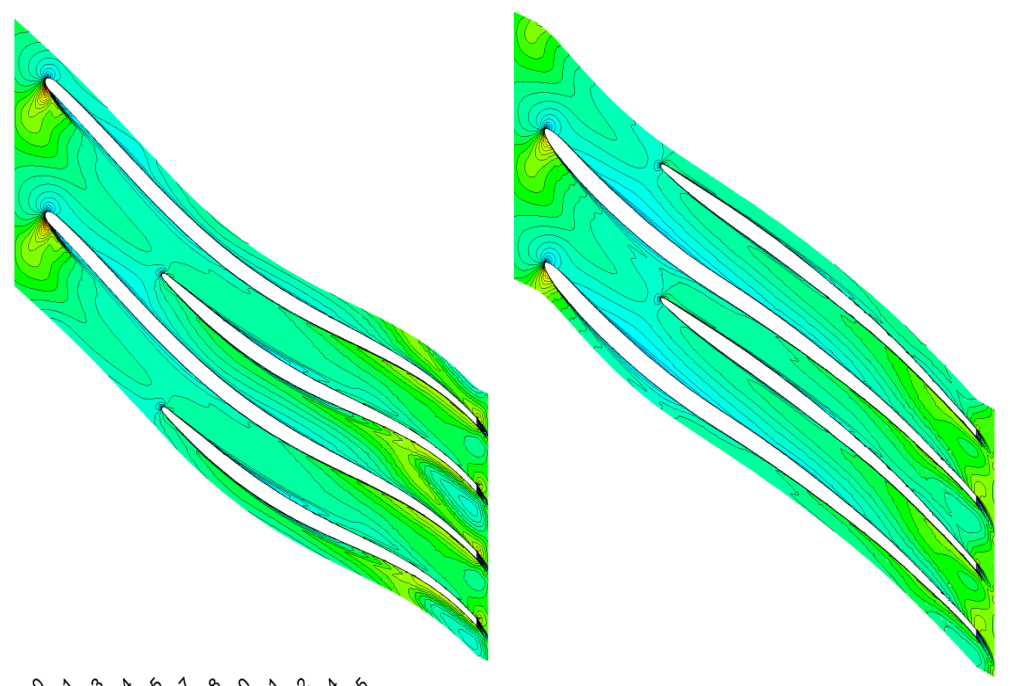

Figure 5.21: Mach number contour comparison between the original (LHS) and redesign (RHS) at $15 \%$ of span

blade channel. The CFD prediction was then compared with the detailed L2F measurement of the flow inside the impeller and good correlation was obtained between the predictions and measurements. Also they found good correlation between predicted and measured pressure ratio for SRV2 impeller with vaneless diffuser tested at 100\%, $80 \%$ and $60 \%$ speeds. The inverse designed impeller was found to have higher pressure ratio and isentropic efficiency at all speeds.

Figure 5.20 revels a comparison of characteristics at the design speed. In order to show how the improved flow field in the blade passages is associated with this aerodynamic performance, we have particularly chosen an operating point of $2.72 \mathrm{~kg} / \mathrm{s}$ mass flow for comparisons. The details of flow physics are given in Figs. 5.21 - 5.25, which are the blade-to-blade views of the Mach number contours at different spanwise positions ranging from 15\% (near the hub) to $95 \%$ (near the shroud). In Figs. 5.26 - 5.30 are shown the comparisons of pressure distributions in the streamwise direction on the main blades and splitters. By observing these figures, a few important insights can be obtained and they explain why the inversely designed compressor is superior.

1. Flow separations at the LE are clearly present on the original compressor 

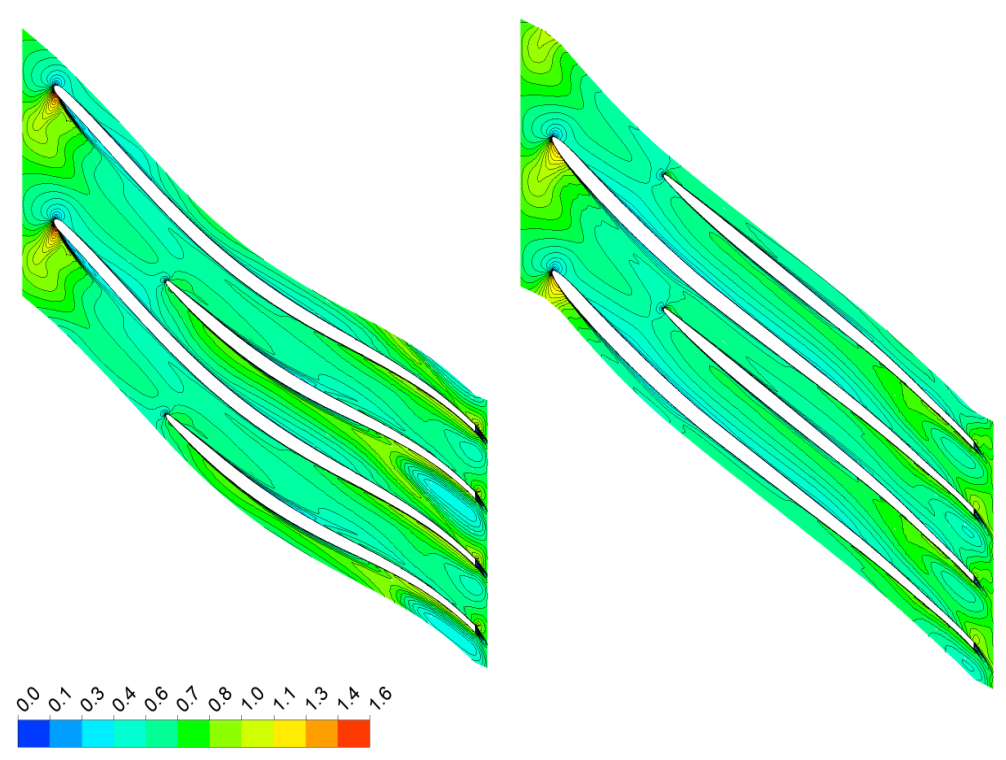

Figure 5.22: Mach number contour comparison between the original (LHS) and redesign (RHS) at $25 \%$ of span

from the lowest spanwise sections whereas they are absent on the redesign until $75 \%$ of the span, and as Figs. 5.24 and 5.25 depict, the flow separation in main blade and splitter channel of inverse design is much weaker than that in the original design. The reduction of flow separation increases the effective passage area, and resultantly weakening the shock strengths.

2. Shocks at the LE of the main blade of the redesigned compressor are significantly weaker than those of the original up to $50 \%$ of the span (Figs. 5.21 - 5.23), and it can also be found in Figs. 5.26 - 5.28 that the original main blade sustains stronger loading near the LE up to the mid of span.

3. Shocks formed between the PS of main blade and SS of splitter in the redesigned compressor are weaker those in the original one at all spanwise positions. This is also confirmed by the blade loading distributions. It can be seen in Figs. 5.21 - 5.25 that the pressure loading near the LE of the inverse designed splitter is smaller than that of the original splitter, and this phenomenon is quite obviously shown in Figs. 5.28 to 5.30 from 0.2 to 0.4 streamwise position.

Figure 5.31 shows that the hub-to-shroud Mach number contour details at 

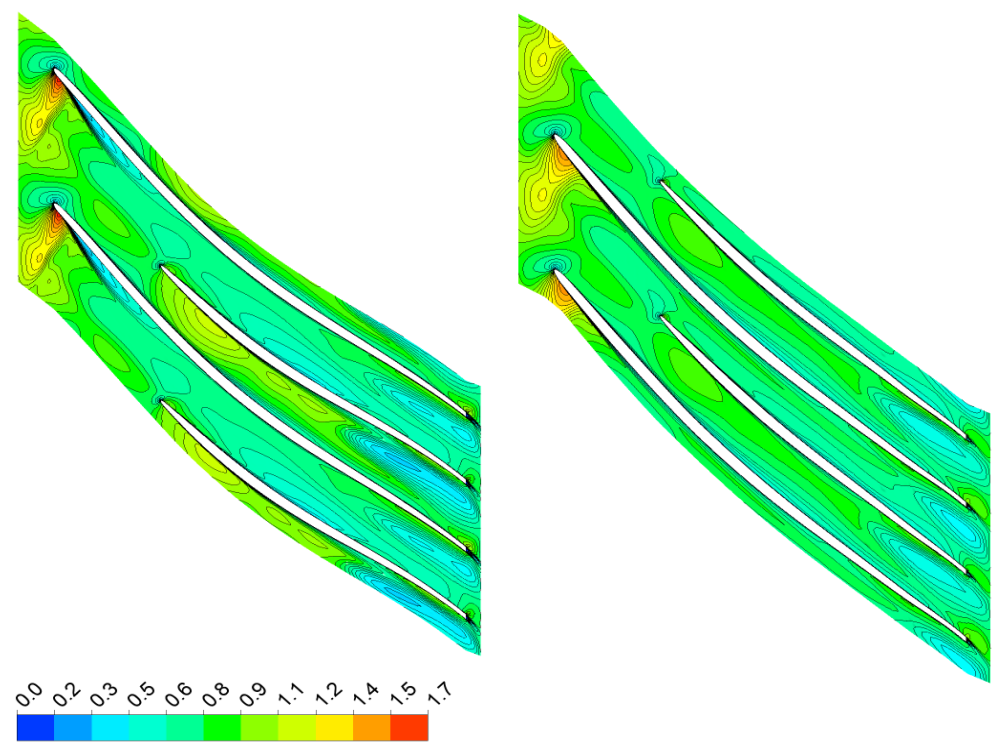

Figure 5.23: Mach number contour comparison between the original (LHS) and redesign (RHS) at $50 \%$ of span

the leading edges of two compressors. It confirms that shocks near the hub of the redesign are weaker than the original one; however, this phenomenon did not continue all the way to the shroud. The shocks caused by the redesign after $50 \%$ of the span have larger strength than those of the original. This is further validated by Fig. 5.32, which shows the efficiency variations at the TE from hub to shroud. It can be clearly seen that the inverse design has higher efficiencies up to $60 \%$ of the span. Figure 5.33 demonstrates the efficiency and static entropy variations of two compressors from the impeller inlet to outlet. It can be seen that the redesigned impeller generated less static entropy than the original design, which consequently results in a higher efficiency.

The CFD simulations show that the redesigned impeller generates weaker shocks in the inducer and significantly reduces the flow separation. The weaker shock structure noticeably reduces the generated noise. 

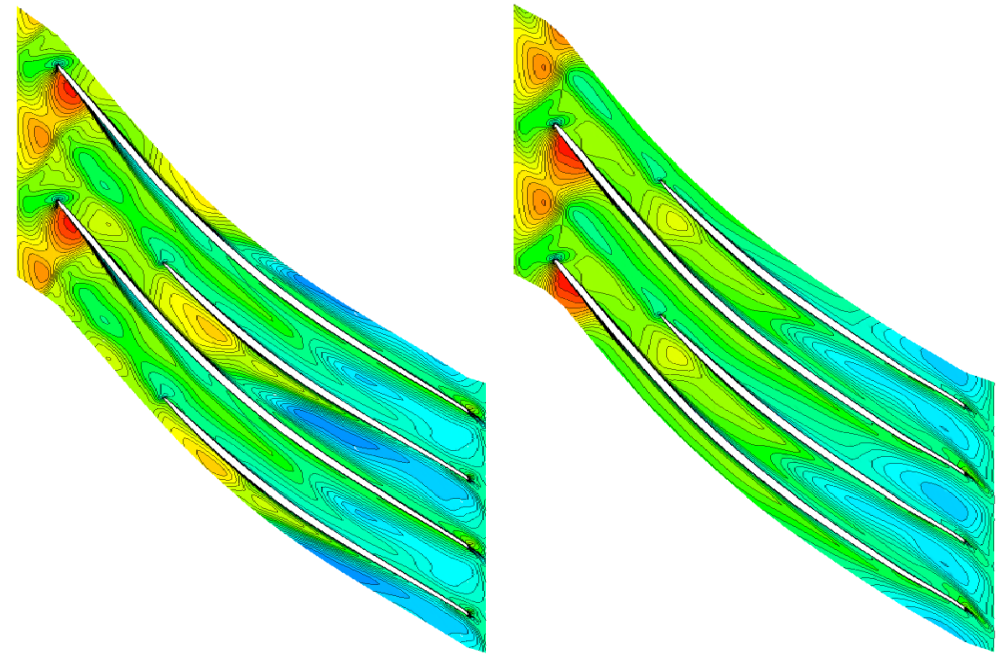

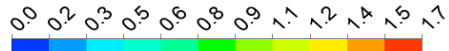

Figure 5.24: Mach number contour comparison between the original (LHS) and redesign (RHS) at $75 \%$ of span
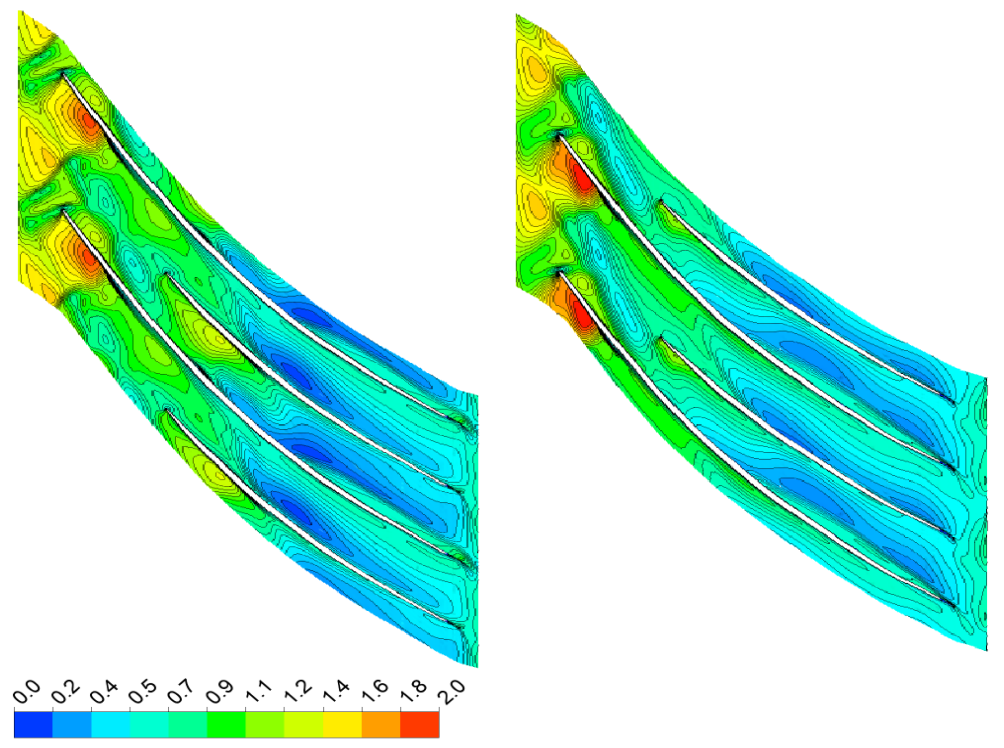

Figure 5.25: Mach number contour comparison between the original (LHS) and redesign (RHS) at $95 \%$ of span 

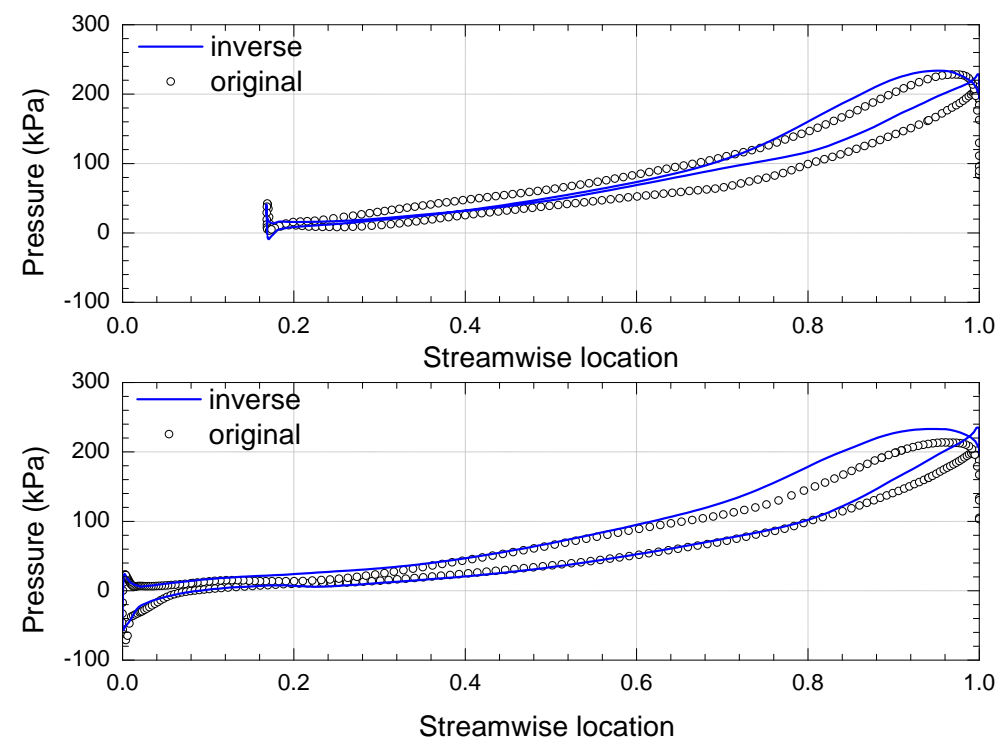

Figure 5.26: Streamwise blade loading distributions on the main blade (lower) and splitter (upper) at $15 \%$ of span
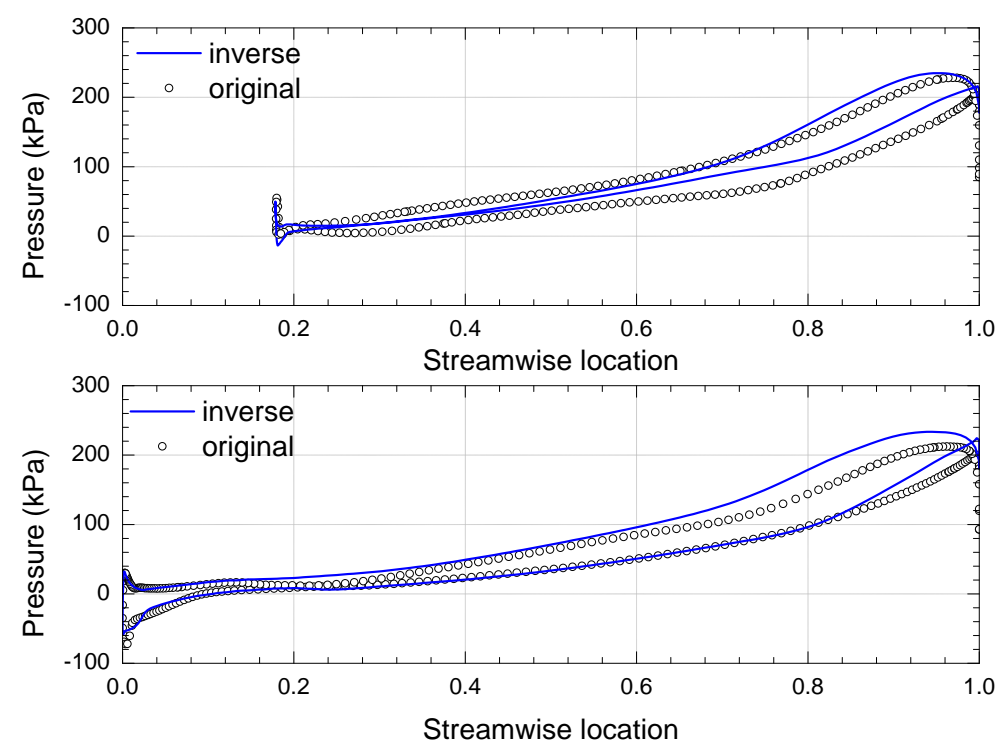

Figure 5.27: Streamwise blade loading distributions on the main blade (lower) and splitter (upper) at $25 \%$ of span 

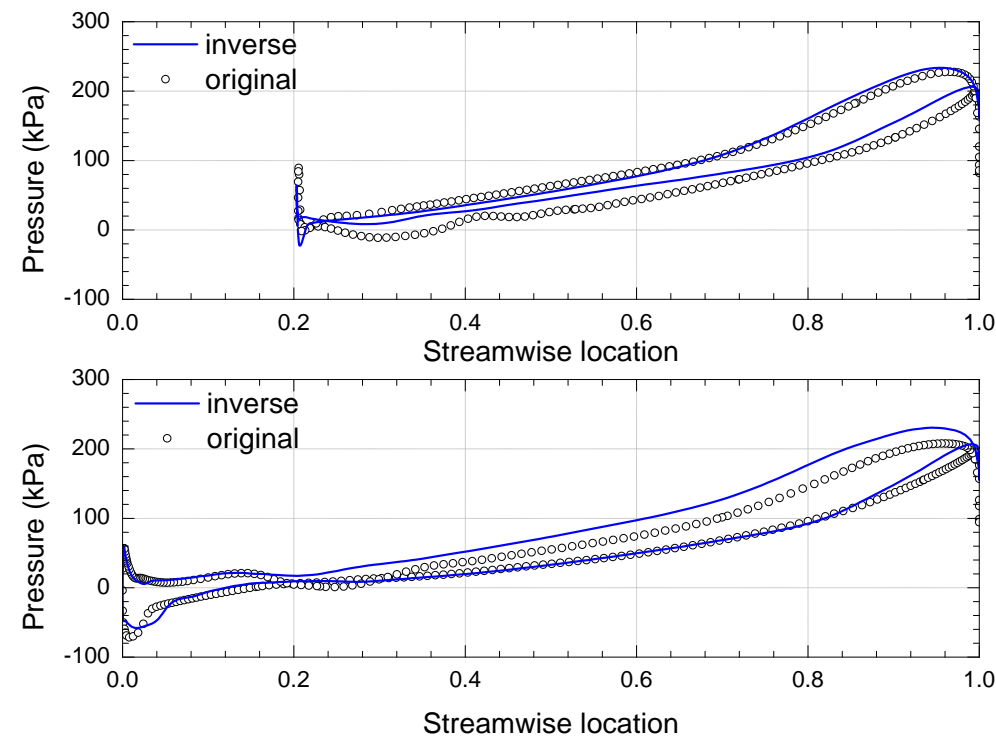

Figure 5.28: Streamwise blade loading distributions on the main blade (lower) and splitter (upper) at $50 \%$ of span
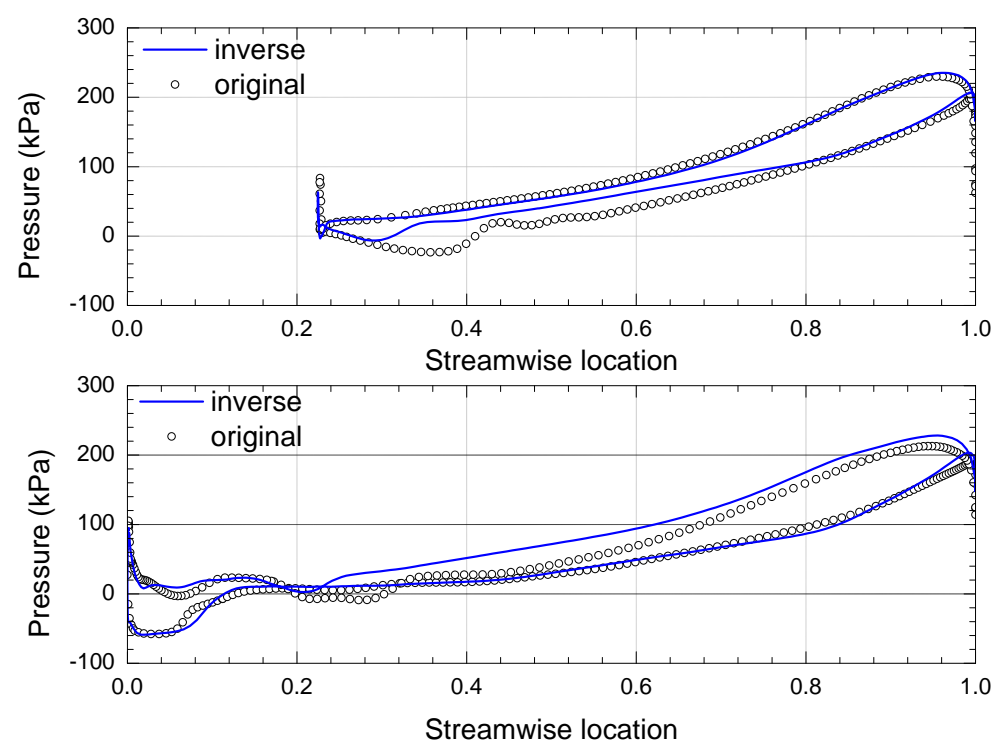

Figure 5.29: Streamwise blade loading distributions on the main blade (lower) and splitter (upper) at $75 \%$ of span 

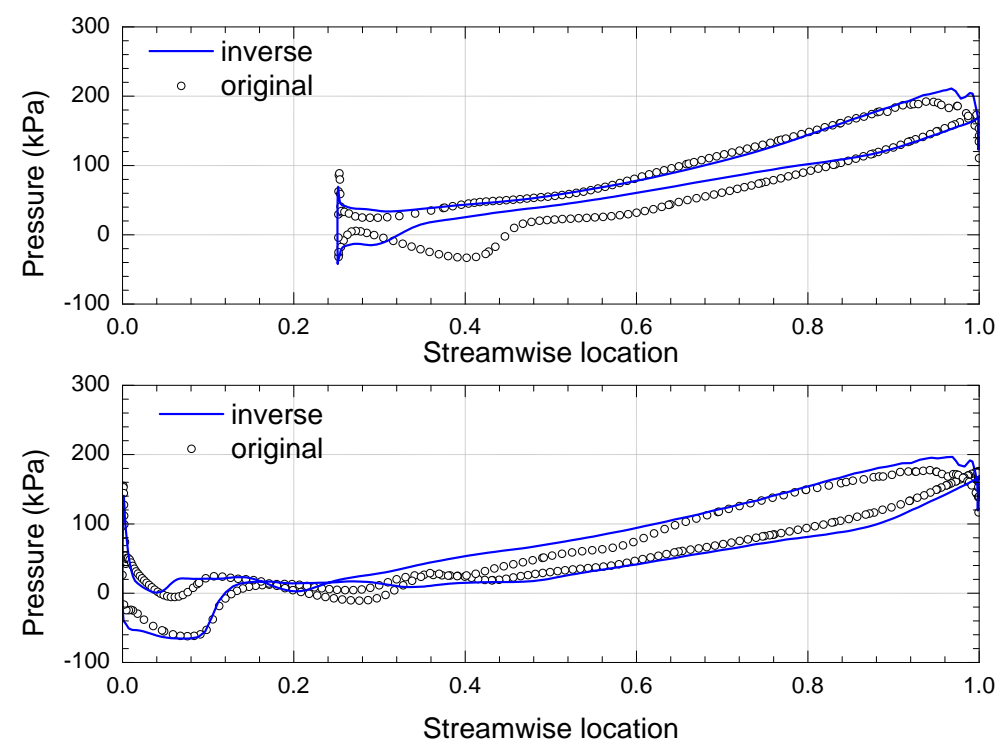

Figure 5.30: Streamwise blade loading distributions on the main blade (lower) and splitter (upper) at $95 \%$ of span
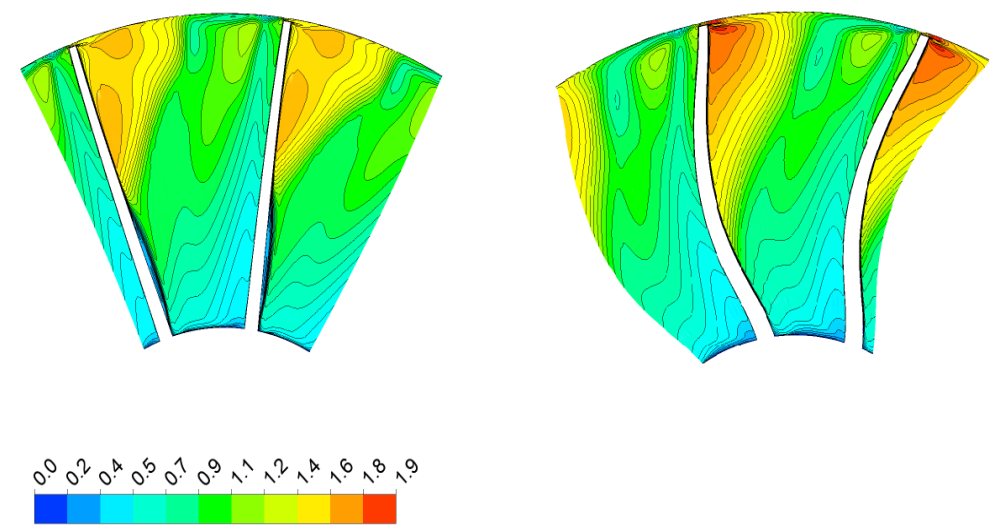

Figure 5.31: Mach number contour comparison between the original (LHS) and redesign (RHS) at $\mathrm{LE}$ 


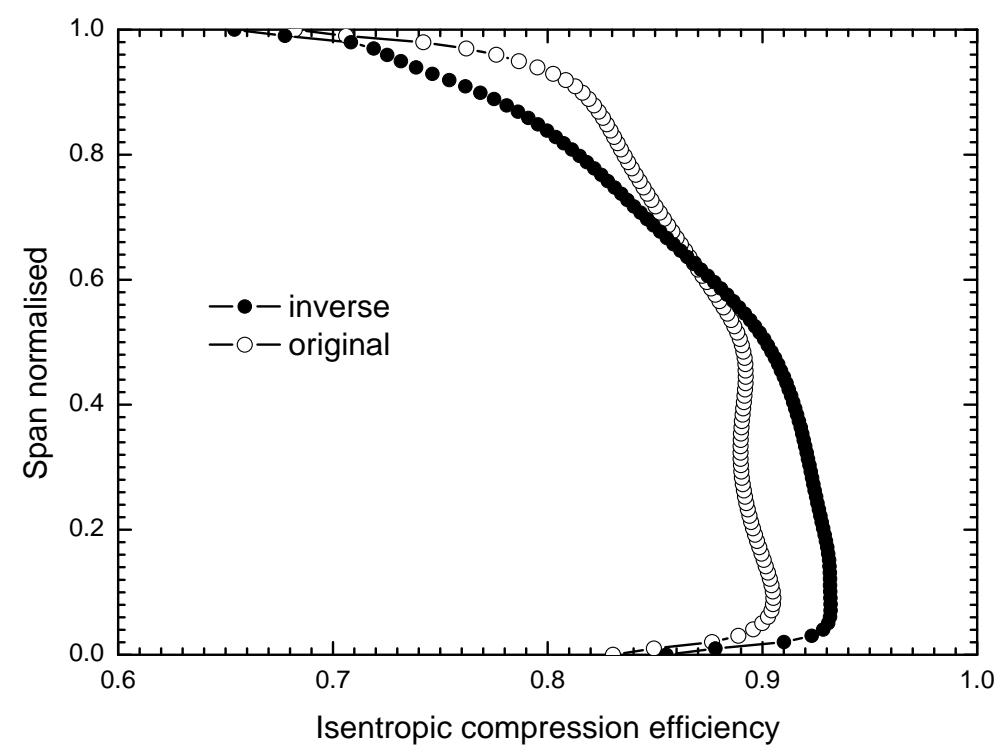

Figure 5.32: Comparison of spanwise variation of isentropic compression efficiency at the TE
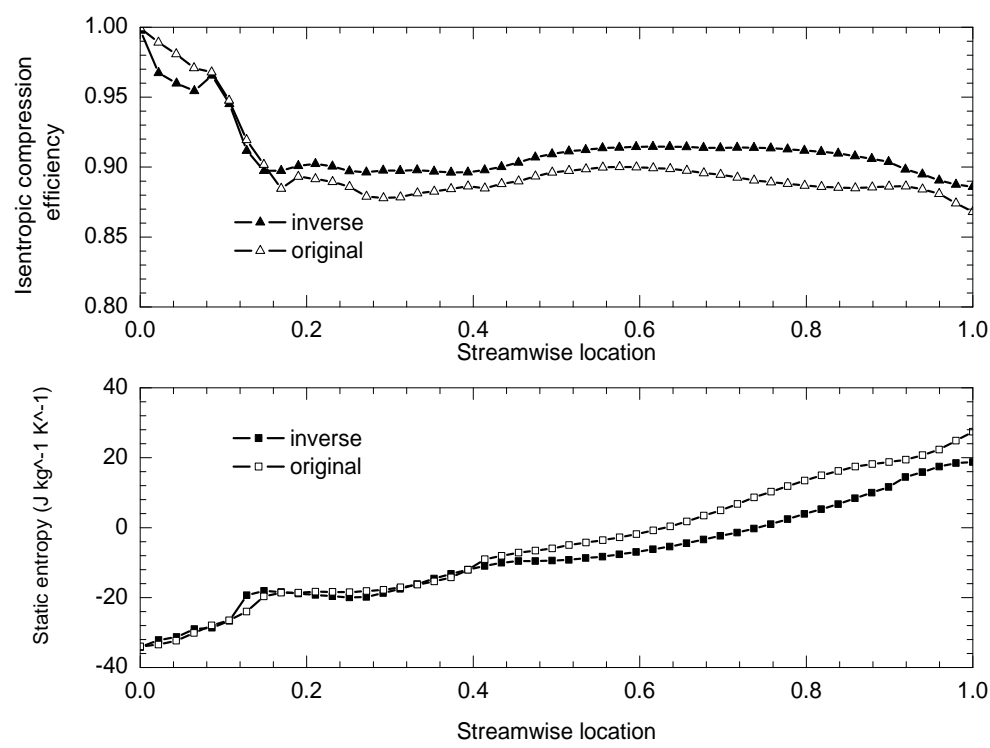

Figure 5.33: Comparison of isentropic compression efficiency in streamwise location from impeller inlet to outlet 
"All theory is grey, my friend. But forever green is the tree of life."

Johann Wolfgang von Goethe (1749 - 1832)

\section{Mathematical formulations of the inverse design method}

In the last chapter, a transonic centrifugal impeller designed by the inverse design code, TURBOdesign1 (abbreviated as TD1 henceforth) was presented. In the work of Zangeneh et al. [2011] and Wang \& Zangeneh [2014], they have showed that the redesigned impeller has significant improvements in terms of aerodynamics and aeroacoustics. The same design approach will be adopted in this thesis.

In the present chapter, the theory of inverse design method will be explained for the thoroughness of this thesis. We shall present some of the key derivations of the mathematical formations used in this approach. 


\subsection{Theoretical formulation of inverse design}

The inverse design method for aerofoils or turbomachinery blade has a long history. We have reviewed in detail in Chapter 2 about the previous work dedicated to this field. In this section, the fundamentals of theoretical formulations used in TD1 will be elaborated to some extent so that one can understand better the design approach used in the present work.

The foundation of the inverse method used in TD1 was completed in a number of classical works by many researchers at Cambridge University and MIT, such as Borges [1990a,b]; Hawthorne et al. [1984]; Tan et al. [1984]; Tjokroaminata [1992]; Yang [1991]; Zangeneh [1991]. The theory behind it involves a great deal of very sophisticated and complicated mathematical treatment. It is not the intention of this thesis to re-derive all the equations used in TD1. The intention here is to present a series of consistent formulations that fit the current thesis.

The primary feature of the inverse design method in this thesis is to prescribe the exit flow's angular momentum/swirl velocity, $r \overline{V_{\theta}}$ and blade loading, which is the static pressure difference between suction and pressure surface. Among many different inverse design approaches, the present method is a very convenient and powerful one for the design of centrifugal machines for its robustness, easiness of convergence and direct association with the compressor's capability of increasing the gas pressure and its efficiency.

Let us begin with turbomachinery Euler equation (see Dixon \& Hall [2010]),

$$
\Delta H=H_{02}-H_{01}=\dot{m} c_{p}\left(T_{02}-T_{01}\right)=\dot{m} \omega\left[\left(r \overline{V_{\theta}}\right)_{2}-\left(r \overline{V_{\theta}}\right)_{1}\right],
$$

in which

- $c_{p}$ is the specific heat capacity;

- $H$ is the enthalpy;

- $\dot{m}$ is the specific mass flow;

- $r$ is the radius;

- $T$ is the temperature; 
- $V$ is the flow velocity;

- $\omega$ is the rotating speed

- $\theta$ indicates the tangential direction.

The subscript 0 denotes the total quantity, 1 and 2 pertain to the compressor inlet and out respectively. The over bar means that the quantity below is circumferentially averaged. Eq. 6.1 reveals that the enthalpy change, $\Delta H$, of fluid through a compressor is proportional to the change of $r \overline{V_{\theta}}$ assuming the compression process is free of energy loss. It is common to assume that the flow is free of vortex at the inlet, thus, $\left(r \overline{V_{\theta}}\right)_{1}=0$. The higher enthalpy gained by the gas through the compression, the higher pressure ratio it can be. The isentropic compression efficiency can be calculated by,

$$
\eta=\frac{\left(\frac{P_{02}}{P_{01}}\right)^{\frac{\gamma-1}{\gamma}}-1}{\frac{T_{02}}{T_{01}}-1}=\frac{\Pi^{\frac{\gamma-1}{\gamma}}-1}{\frac{T_{02}}{T_{01}}-1},
$$

in which $\eta$ is isentropic compression efficiency, $\Pi$ is the pressure ratio $P_{02} / P_{01}$. Using Eq. 6.1 and 6.2 one can get a new equation for $\eta$ shown below,

$$
\eta=\frac{\left(\Pi^{\frac{\gamma-1}{\gamma}}-1\right) c_{p} T_{01}}{\left(\omega r_{2}\right)^{2} r V_{\theta}^{*}}
$$

where $r V_{\theta}^{*}$ is a non-dimensionalised quantity defined as

$$
r V_{\theta}^{*}=\frac{\left(r \overline{V_{\theta}}\right)_{2}}{r_{2}\left(\omega r_{2}\right)}
$$

in which $\omega$ is the rotating speed. Eq. 6.3 correlates $\eta$ and $\Pi$ with $r \overline{V_{\theta}}$ and all the other parameters in this equation are gas constants. This equation shows the significance of $r \overline{V_{\theta}}$ and an appropriate choice of this quantity is quite essential for determining a compressor's performances in a macro perspective. In the following part of this section, we will show how $r \overline{V_{\theta}}$ is related to the blade loading and how it can determine the three dimensional geometry of a turbomachine. 


\subsubsection{Blade loading}

This subsection focuses on elaborating how the blade loading is related to the quantity $r \overline{V_{\theta}}$. Figure 6.1 shows an array of full and splitter blades whose thickness is infinitely small so that they can be represented by two groups of vortex sheets in the B2B (blade to blade frame) frame, i.e., $M^{\prime}-\theta$ frame. The vortex sheets are defined by

$$
\alpha^{j}=\theta^{j}-f^{j}(r, z) \text {, }
$$

in which the superscript $j$ denotes the full $(j=1)$ and splitter $(j=2)$ blade. $\alpha^{j}$ is also the camber line profile for the blade with finite thickness. The flow properties going through the blade channel are subject to the following assumptions:

1. The inlet flow is steady, inviscid, and uniform. The only vorticity is therefore the bound vorticity on the blade surface.

2. No trailing edge shed vorticity is present.

3. The blade has infinitely thin thickness so that blade row can be represented by an array of vortex sheet. However, to account for the blade blockage effects a mean stream surface thickness parameter is used in the continuity equation of the mean flow.

The bound vorticity, $\Gamma_{b}^{j}$ is calculated by the circular integration

$$
\Gamma_{b}^{j}=\oint_{c} \boldsymbol{V}^{j} \cdot d \boldsymbol{l}_{m r}^{j}
$$

in which $d \boldsymbol{l}$ is the elementary vector along the blade streamline and the subscript $m r$ denotes the vector quantity pertaining to it is along the streamwise or merdional direction. Using Stoke's theorem yields

$$
\Gamma_{b}^{j}=\oint_{c} \boldsymbol{V}^{j} \cdot d \boldsymbol{l}_{m r}^{j}=\iint_{\partial A^{j}}\left[\nabla \times \boldsymbol{V}^{j}\right] \cdot d \boldsymbol{l}_{m r}^{j} w_{d}
$$

where $w_{d}$ is the unit width of the sheet.

Differentiating Eq. 6.7 gives,

$$
d \Gamma_{b}^{j}=\left(\nabla \times \boldsymbol{V}^{j}\right) \cdot d \boldsymbol{l}_{m r}^{j} w_{d}
$$




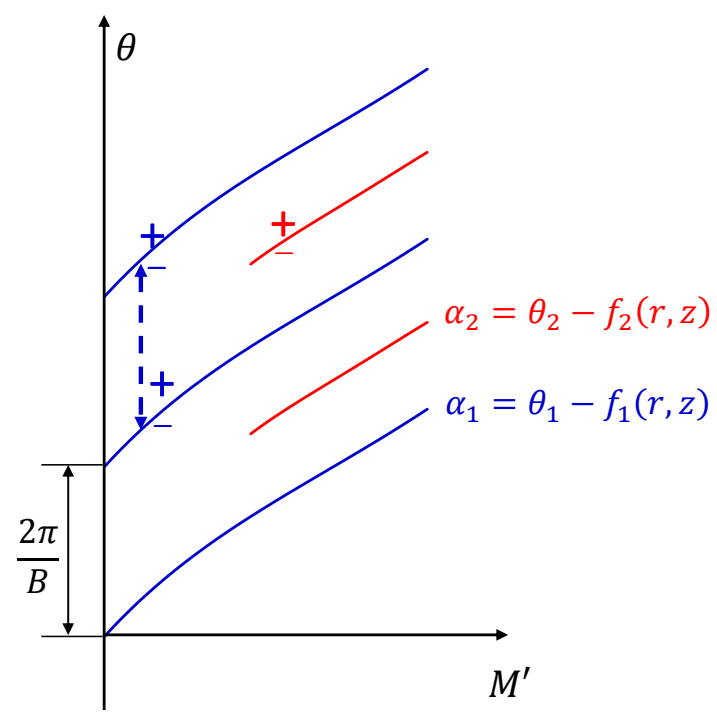

Figure 6.1: Cascade notaitons

The Kutta-Joukowski lift force theorem in the vector form is

$$
d \boldsymbol{F}^{j}=\rho^{j} \boldsymbol{V}_{b l}^{j} \times d \boldsymbol{\Gamma}_{b}^{j}
$$

in which the subscript $b l$ denotes the quantity is on the blade surface. The lift force per unit width $\boldsymbol{F}^{j}$ is related to the blade loading $\Delta p^{j}=\left(p^{+}-p^{-}\right)^{j}$ through

$$
d \boldsymbol{F}^{j}=\Delta p^{j} \nabla \alpha^{j} d l_{m r}^{j}=\rho^{j} \boldsymbol{V}_{b l}^{j} \times\left(\nabla \times \boldsymbol{V}^{j}\right) \cdot d \boldsymbol{l}_{m r}^{j},
$$

in which $\nabla \times \boldsymbol{V}^{j}=\Omega^{j}$, is the vorticity vector field. For the entire array of vortex sheet, the blade loading can be generalised by using the periodic Dirac delta function, $\delta_{p}\left(\alpha^{j}\right)$,

$$
\Delta p^{j} \nabla \alpha^{j} \delta_{p}\left(\alpha^{j}\right)=\frac{2 \pi}{B} \rho^{j} \boldsymbol{V}_{b l}^{j} \times\left(\nabla \times \boldsymbol{V}^{j}\right)=\frac{2 \pi}{B} \rho^{j} \boldsymbol{V}_{b l}^{j} \times \Omega^{j}
$$

It will be shown later the vorticity vector $\Omega^{j}$ can be represented by

$$
\mathbf{\Omega}^{j}=\overline{\boldsymbol{\Omega}}^{j} \delta_{p}\left(\alpha^{j}\right)=\left[\nabla\left(r{\overline{V_{\theta}}}^{j}\right) \times \nabla \alpha^{j}\right] \delta_{p}\left(\alpha^{j}\right),
$$


in which the over bar "-" defines a tangential mean,

$$
\overline{A r}(r, z)=\frac{B}{2 \pi} \int_{0}^{2 \pi / B} A r(r, \theta, z) d \theta
$$

where $A r$ is an arbitrary variable.

Substituting Eq. 6.12 into Eq. 6.11 leads to

$$
\Delta p^{j}=\left(p^{+}-p^{-}\right)^{j}=\frac{2 \pi}{B} \rho^{j} \boldsymbol{V}_{b l}^{j} \cdot \nabla\left(r V_{\theta}\right)^{j}
$$

which has implied

$$
\boldsymbol{V}_{b l}^{j} \times\left(\nabla\left(r{\overline{V_{\theta}}}^{j}\right) \times \nabla \alpha^{j}\right)=\left(\boldsymbol{V}_{b l}^{j} \cdot \nabla \alpha^{j}\right) \nabla\left(r{\overline{V_{\theta}}}^{j}\right)-\left[\boldsymbol{V}_{b l}^{j} \cdot \nabla\left(r{\overline{V_{\theta}}}^{j}\right)\right] \nabla \alpha^{j}
$$

in which the non-permeable/Neumann boundary condition on the blade surface requires,

$$
\boldsymbol{V}_{b l}^{j} \cdot \nabla \alpha^{j}=0
$$

In Eq. 6.14 the dot product between $\boldsymbol{V}_{b l}^{j}$ and $\nabla\left(r \overline{V_{\theta}}\right)^{j}$ is along the meridional projection of the blade streamline, thus Eq. 6.14 is reduced to

$$
\Delta p^{j}=\left(p^{+}-p^{-}\right)^{j}=\frac{2 \pi}{B} \rho^{j} V_{m b l}^{j} \frac{\partial\left(r \bar{V}_{\theta}^{j}\right)}{\partial m^{j}}
$$

where $m$ is the distance along the meridional projection of the blade streamline and $V_{m b l}$ is the meridional velocity of the flow on the blade surface. Let us remind ourselves that,

$$
\boldsymbol{V}_{b l}=\boldsymbol{W}_{b l}+\boldsymbol{\omega} \times \boldsymbol{r}
$$

in which $W_{b l}$ is relative velocity vector on the blade surface, $\boldsymbol{\omega}$ is the angular velocity vector, and $\boldsymbol{r}$ is the radial vector. The second term on Eq. 6.18 only has component in $\hat{\boldsymbol{\theta}}$ direction, thus, its projection on the meridional direction is zero. Therefore, $V_{m b l}=W_{m b l}$ and Eq. 6.17 is rewritten as

$$
\Delta p^{j}=\left(p^{+}-p^{-}\right)^{j}=\frac{2 \pi}{B} \rho^{j} W_{m b l}^{j} \frac{\partial\left(r{\overline{V_{\theta}}}^{j}\right)}{\partial m^{j}} .
$$




\section{MATHEMATICAL FORMULATIONS OF THE INVERSE DESIGN METHOD}

Eq. 6.19 is valid for both incompressible and compressible flows. For an incompressible flow, the pressure loading can be determined by the derivative term in the RHS since the density $\rho$ is known from the flow property and remains constant. In case of a compressible flow, both $\rho$ and $\partial\left(r \overline{V_{\theta}}\right) / \partial m$ must be known a priori in order to prescribe the pressure loading. But the density is iteratively computed rather than a known input parameter, thus, a different loading prescription is required for the compressible flow condition.

Thanks to the isentropic assumption, the enthalpy of the working flow can be written as

$$
H=U+p Q
$$

where $U$ is the internal energy and $Q$ is the volume. Differentiating both sides of eq. 6.20 yields,

$$
d H=d U+d(p Q)=d U+Q d p+p d Q
$$

in which $d U=T d s-p d Q$. Thus,

$$
d H=d U+d(p Q)=T d s-p d Q+Q d p+p d Q=T d s+Q d p
$$

in which $T d S=0$ due to the isentroic assumption. Eq. 6.22 is equivalent to

$$
d h=\frac{d p}{\rho} .
$$

Inserting Eq. 6.23 into Eq. 6.19 gives,

$$
\Delta h^{j}=\left(h^{+}-h^{-}\right)^{j}=\frac{2 \pi}{B} W_{m b l}^{j} \frac{\partial\left(r \bar{V}_{\theta}^{j}\right)}{\partial m^{j}},
$$

which shows that the specific enthalpy jump can be controlled by the derivative term $\partial\left(r \overline{V_{\theta}}\right) / \partial m$. Eq. 6.24 is therefore used for the compressible flow. 


\subsubsection{Flow solver: vorticity method and Clebsch formula- tion}

In the last subsection, equations that govern the blade loading for both incompressible and compressible flows have been derived. We will only focus on the compressible flow case since it is a compressor to be designed. Eq. 6.24 suggests that the enthalpy jump is associated with meridional velocity on and the swirl velocity's derivative at the meridional direction. It is quite natural to see that the two quantities are needed in order to prescribe a enthalpy jump distribution, and the density is needed to further get the pressure loading distribution. Unlike some other inverse design work, such as Tiow et al. [2002], the 3D meshes are needed for the blade channel first, and then either Euler solver is used to calculate the flow field. The current inverse method does not require a 3D mesh but only a $2 \mathrm{D}$ mesh in the meridional plane. At a spanwise height the upper and lower surface profiles are computed individually subject to the enthalpy jump specified on them. In this subsection, we will present how the flow solver used in the current inverse method calculates the density and velocity field, and thus the pressure jump can be further computed using Eq. 6.19.

\subsubsection{Calculation of the density}

Let us assume the flow is steady everywhere in the turbomachine and there is no energy loss, then we can write

$$
\frac{D H^{r o t}}{D t}=0
$$

which can be written into the following form by expanding the total derivative,

$$
\boldsymbol{W} \cdot \nabla H^{r o t}=0
$$

where we have used the fact $D / D t=\partial / \partial t+\boldsymbol{W} \cdot \nabla$ and $\partial H^{r o t} / \partial t=0$ since the flow is steady. $\boldsymbol{W}$ is the relative velocity vector and $H^{r o t}$ is the rothalpy (see $\mathrm{Wu}$ 
[1952]) that can be expanded as,

$$
H^{r o t}=h+\frac{1}{2} \boldsymbol{V} \cdot \boldsymbol{V}-\omega r \overline{V_{\theta}}=h+\frac{1}{2} \boldsymbol{W} \cdot \boldsymbol{W}-\frac{1}{2} \omega r^{2}=\text { const. }
$$

It has also been assumed that the flow is uniform at the inlet, therefore, the rothalpy $H^{\text {rot }}$ remains as a constant. For a perfect gas, Eq. 6.27 can be written as,

$$
c_{p} T^{r o t}=c_{p} T+\frac{1}{2}(\boldsymbol{W} \cdot \boldsymbol{W})-\frac{1}{2} \omega^{2} r^{2},
$$

which can be cast into this form,

$$
\frac{T}{T^{r o t}}=1+\frac{\omega^{2} r^{2}-\boldsymbol{W} \cdot \boldsymbol{W}}{2 c_{p} T^{r o t}} .
$$

For a isentropic flow process, the density has the following relationship with temperature,

$$
\frac{\rho}{\rho^{r o t}}=\left(\frac{T}{T^{r o t}}\right)^{1 /(\gamma-1)} .
$$

Combining Eq. 6.29 and 6.30 yields,

$$
\frac{\rho}{\rho^{r o t}}=\left[1+\frac{\omega^{2} r^{2}-\boldsymbol{W} \cdot \boldsymbol{W}}{2 c_{p} T^{r o t}}\right]^{1 /(\gamma-1)}
$$

Once the flow vector field $\boldsymbol{W}$ is solved, Eq. 6.29 can be used to compute the static temperature, and as a result, the density can be worked out in Eq. 6.30.

\subsubsection{Calculation of the velocity - approximate method}

\section{The continuity equation}

In this subsection, an approximate method to calculate the velocity vector field will be explained. It is called an approximate method because the density variation in the pitchwise direction is neglected. The velocity field is decomposed into a mean and periodic part, i.e.,

$$
\boldsymbol{W}=\boldsymbol{V}-\boldsymbol{\omega} \times \boldsymbol{r}=\overline{\boldsymbol{V}}+\tilde{\boldsymbol{v}}-\boldsymbol{\omega} \times \boldsymbol{r}
$$


where "-" and " " pertain to the mean and periodic component respectively. The continuity equation in the reference frame is,

$$
\nabla \cdot(\rho \boldsymbol{W})=0
$$

Taking $\overline{\boldsymbol{W}}=\boldsymbol{W}-\boldsymbol{\omega} \times \boldsymbol{r}$, Eq. 6.33 can be re-arranged to

$$
\nabla \cdot \overline{\boldsymbol{W}}=-\overline{\boldsymbol{W}} \nabla \ln \bar{\rho}
$$

Eq. 6.34 can also rewritten as,

$$
\nabla \cdot(\bar{\rho} \overline{\boldsymbol{W}})=0
$$

To account for the blade blockage due to its thickness a stream thickness factor $B_{f}$ can be included in Eq. 6.35, i.e.,

$$
\nabla \cdot\left(\bar{\rho} B_{f} \overline{\boldsymbol{W}}\right)=0
$$

in which

$$
B_{f}=1-\frac{t_{\theta}}{r} \frac{B}{2 \pi}
$$

$t_{\theta}$ is the tangential thickness ( $\hat{\boldsymbol{\theta}}$ direction) and $r$ is the radius. The thickness normal to the camber line can be obtained from the following identity,

$$
t_{n}=\frac{t_{\theta}}{1+r^{2}\left[\left(\frac{\partial f}{\partial r}\right)^{2}+\left(\frac{\partial f}{\partial z}\right)^{2}\right]}
$$

A stream function $\psi$ (Batchelor [2000]) can be used to describe the mean flow and it can be defined in such a way that

$$
\overline{\boldsymbol{V}}=\overline{V_{r}} \hat{\boldsymbol{r}}+\overline{V_{z}} \hat{\boldsymbol{z}}=-\frac{\rho_{i}}{r B_{f} \bar{\rho}} \frac{\partial \psi}{\partial z} \hat{\boldsymbol{r}}+\frac{\rho_{i}}{r B_{f} \bar{\rho}} \frac{\partial \psi}{\partial r} \hat{\boldsymbol{z}}
$$

where $\rho_{i}$ is a reference density and $\psi$ is the so called Stoke stream function for the axisymmetric flow in the cylindrical coordinates. Taking curl of LHS 
in Eq. 6.39 gives the mean vorticity vector $\bar{\Omega}$, namely,

$$
\overline{\boldsymbol{\Omega}}=\nabla \times \overline{\boldsymbol{V}}=\left(\frac{\partial \overline{V_{r}}}{\partial z}-\frac{\partial \overline{V_{z}}}{\partial r}\right) \hat{\boldsymbol{\theta}}
$$

Using the $\overline{V_{r}}$ and $\overline{V_{z}}$ expressed in terms of $\psi$ in Eq. 6.39 and substituting them in Eq. 6.40 yields,

$$
\begin{aligned}
\overline{\boldsymbol{\Omega}}= & -\frac{\rho_{i}}{B_{f} \bar{\rho} r}\left[\frac{\partial^{2} \psi}{\partial z^{2}}+\frac{\partial \psi}{\partial z}\left(\frac{\partial \ln \left(\bar{\rho} / \rho_{i}\right)}{\partial z}+\frac{\partial \ln \left(1 / B_{f}\right)}{\partial z}\right)\right. \\
& \left.+\frac{\partial^{2} \psi}{\partial r^{2}}-\frac{1}{r} \frac{\partial \psi}{\partial r}+\frac{\partial \psi}{\partial r}\left(\frac{\partial \ln \left(\bar{\rho} / \rho_{i}\right)}{\partial r}+\frac{\partial \ln \left(1 / B_{f}\right)}{\partial r}\right)\right] \hat{\boldsymbol{\theta}}
\end{aligned}
$$

The above equation shows the vorticity field of a mean flow field, and it is a general expression including the bound vorticity on the blade surface. The flow field is free of vorticity everywhere else but on the blade surface, i.e.,

$$
\begin{aligned}
& \frac{\partial^{2} \psi}{\psi z^{2}}+\frac{\partial \psi}{\partial z}\left(\frac{\partial \ln \left(\bar{\rho} / \rho_{i}\right)}{\partial z}+\frac{\partial \ln \left(1 / B_{f}\right)}{\psi z}\right)+ \\
& \frac{\partial^{2} \psi}{\psi r^{2}}+\frac{\partial \psi}{\partial r}\left(\frac{\partial \ln \left(\bar{\rho} / \rho_{i}\right)}{\partial r}+\frac{\partial \ln \left(1 / B_{f}\right)}{\partial r}\right)-\frac{1}{r} \frac{\partial \psi}{\psi r}=0
\end{aligned}
$$

which is an elliptic differential equation that can be solved using the boundary conditions at two endwalls, upstream, and downstream.

- End wall boundary conditions at hub and shroud. On the hub and shroud wall, the velocity components normal to the hub and shroud contour must vanish. Thus,

$$
\begin{aligned}
& \overline{\boldsymbol{V}} \cdot \boldsymbol{n}_{h}=0, \\
& \overline{\boldsymbol{V}} \cdot \boldsymbol{n}_{s}=0,
\end{aligned}
$$

in which $\boldsymbol{n}_{h}$ and $\boldsymbol{n}_{s}$ are the normal vectors to the hub and shroud.

- Far upstream velocity boundary condition The far upstream velocity is a design specification, i.e., the inlet velocity $\overline{\boldsymbol{V}}_{-\infty}$ is given 
as a uniform constant a priori.

$$
-\frac{1}{r} \frac{\rho_{i}}{\bar{\rho}} \frac{\partial \psi}{\partial m}=\overline{\boldsymbol{V}}_{-\infty} \cdot \boldsymbol{n}_{\text {inlet }}
$$

Using the boundary conditions described by Eq. 6.43, 6.44 and 6.45, Eq. 6.42 can be solved and therefore the mean flow velocity field $\overline{\boldsymbol{V}}$ is obtained. The only unknown left in Eq. 6.32 is the periodic velocity $\tilde{\boldsymbol{v}}$. Next, we will show how to use the surface vorticity method and Clebsch formulation to calculate the periodic component.

2. The surface vorticity method and Clebsch decomposition for periodic flow

It is the assumed that the incoming flow is inviscid, steady, and irrotational. The only vorticity is the bound vorticity on the full and splitter blade surfaces. Thus, the total vorticity, $\Omega$ is

$$
\boldsymbol{\Omega}=\sum_{j=1}^{2} \boldsymbol{\Omega}^{j}=\sum_{j=1}^{2} \overline{\boldsymbol{\Omega}}^{j} \delta_{p}\left(\alpha^{j}\right) .
$$

Using the Clebsch formulation suggested by Lamb [1975]; Wu et al. [2006], one may decompose the velocity vector $\boldsymbol{V}^{j}$ to a potential and a rotational part, therefore,

$$
\boldsymbol{V}^{j}=\nabla \phi^{j}+\lambda^{j} \nabla \mu^{j}
$$

where $\phi(r, \theta, z), \lambda(r, \theta, z)$ and $\mu(r, \theta, z)$ are the Clebsch scalars. Taking curl of Eq. 6.47 yields the vorticity vector $\Omega^{j}$

$$
\begin{aligned}
\boldsymbol{\Omega}^{j} & =\nabla \times \boldsymbol{V}^{j}=\nabla \times\left(\nabla \phi^{j}+\lambda^{j} \nabla \mu^{j}\right) \\
& =\nabla \times \nabla \phi^{j}+\left[\lambda^{j} \nabla \times\left(\nabla \mu^{j}\right)+\left(\nabla \lambda^{j}\right) \times\left(\nabla \mu^{j}\right)\right],
\end{aligned}
$$

in which $\nabla \times \nabla \phi^{j}=0$ and $\nabla \times \nabla \mu^{j}=0$, leaving us

$$
\mathbf{\Omega}^{j}=\left(\nabla \lambda^{j}\right) \times\left(\nabla \mu^{j}\right)
$$


The mean vorticity vector may be written as

$$
\overline{\boldsymbol{\Omega}}^{j}=\nabla \times \overline{\boldsymbol{V}}^{j}=\nabla \bar{\lambda}^{j} \times \nabla \bar{\mu}^{j}
$$

Since the vorticity is the bound vorticity on the blade surface, it must be normal to the gradient of the blade surface $\nabla \alpha^{j}$. Hence, the vorticity can be written as a product of two vectors, one of which is $\nabla \alpha^{j}$, and it must be grad of the scalar $\mu^{j}$. The other vector factor should be $r \bar{V}_{\theta}^{j}$.

Using Eq. 6.50

$$
\begin{aligned}
\overline{\boldsymbol{\Omega}}^{j}=\nabla \times \overline{\boldsymbol{V}}^{j}= & \left(\frac{1}{r} \frac{\partial \bar{V}_{z}^{j}}{\partial \theta}-\frac{\partial \bar{V}_{\theta}^{j}}{\partial z}\right) \hat{\boldsymbol{r}}+\left(\frac{\partial \bar{V}_{r}^{j}}{\partial \bar{V}_{z}^{j}}-\frac{\partial \bar{V}_{z}^{j}}{\partial \bar{V}_{r}^{j}}\right) \hat{\boldsymbol{\theta}} \\
& +\frac{1}{r}\left(\frac{\partial\left(r \bar{V}_{\theta}\right)^{j}}{\partial r}-\frac{\partial \bar{V}_{r}^{j}}{\partial \theta}\right) \hat{\boldsymbol{z}}
\end{aligned}
$$

Since the mean is taken in the tangential direction, $\partial() / \partial \theta=0$

$$
\overline{\boldsymbol{\Omega}}^{j}=\nabla \times \overline{\boldsymbol{V}}^{j}=\left(-\frac{1}{r} \frac{\partial\left(r \bar{V}_{\theta}\right)^{j}}{\partial z}\right) \hat{\boldsymbol{r}}+\left(\frac{\partial \bar{V}_{r}^{j}}{\partial \bar{V}_{z}^{j}}-\frac{\partial \bar{V}_{z}^{j}}{\partial \bar{V}_{r}^{j}}\right) \hat{\boldsymbol{\theta}}+\frac{1}{r} \frac{\partial\left(r \bar{V}_{\theta}\right)^{j}}{\partial r} \hat{\boldsymbol{z}}
$$

Expanding Eq. 6.50 directly leads to

$$
\begin{aligned}
\nabla \mu^{j} & =-\frac{\partial f^{j}}{\partial r} \hat{\boldsymbol{r}}+\frac{1}{r} \hat{\boldsymbol{\theta}}-\frac{\partial f^{j}}{\partial z} \hat{\boldsymbol{z}} \\
\nabla \lambda^{j} & =\frac{\partial \lambda^{j}}{\partial r} \hat{\boldsymbol{r}}+\frac{1}{r} \frac{\partial \lambda^{j}}{\partial \theta} \hat{\boldsymbol{\theta}}+\frac{\partial \lambda^{j}}{\partial z} \hat{\boldsymbol{z}} \\
\overline{\boldsymbol{\Omega}}^{j} & =\nabla \bar{\lambda}^{j} \times \nabla \bar{\mu}^{j} \\
& =-\frac{1}{r} \frac{\partial \bar{\lambda}^{j}}{\partial z} \hat{\boldsymbol{r}}+\left(\frac{\partial \bar{\lambda}^{j}}{\partial r} \frac{\partial f^{j}}{\partial z}-\frac{\partial \bar{\lambda}^{j}}{\partial z} \frac{\partial f^{j}}{\partial r}\right) \hat{\boldsymbol{\theta}}+\frac{1}{r} \frac{\partial \bar{\lambda}^{j}}{\partial r} \hat{\boldsymbol{z}}
\end{aligned}
$$

It is obvious that

$$
\lambda=r \overline{V_{\theta}} \quad \& \quad \lambda^{j}=\left(r \overline{V_{\theta}}\right)^{j}
$$

by comparing the $\hat{\boldsymbol{r}}$ and $\hat{\boldsymbol{z}}$ components in Eq. 6.52 with Eq. 6.53. Finally,

$$
\boldsymbol{\Omega}=\sum_{j=1}^{2} \boldsymbol{\Omega}^{j}=\sum_{j=1}^{2} \overline{\boldsymbol{\Omega}}^{j} \delta_{p}\left(\alpha^{j}\right)=\sum_{j=1}^{2}\left(\nabla\left(r \bar{V}_{\theta}\right)^{j} \times \nabla \alpha^{j}\right) \delta_{p}\left(\alpha^{j}\right) .
$$


Let us recall Eq. 6.32 we have

$$
\boldsymbol{V}^{j}=\overline{\boldsymbol{V}}^{j}+\tilde{\boldsymbol{v}}^{j}
$$

Consequently, the vorticity field will be,

$$
\Omega^{j}=\bar{\Omega}^{j}+\tilde{\Omega}^{j}
$$

Combining Eq. 6.57 and 6.55 gives

$$
\tilde{\Omega}^{j}=\Omega^{j}-\bar{\Omega}^{j}=\nabla\left(r \bar{V}_{\theta}^{j}\right) \times \nabla \alpha^{j}\left[\delta_{p}\left(\alpha^{j}\right)-1\right]
$$

in which $S^{\prime}\left(\alpha^{j}\right)=\delta_{p}\left(\alpha^{j}\right)-1$ shown by Lighthill [1967] and $S^{\prime}\left(\alpha^{j}\right)$ is a periodic sawtooth function defined as

$$
S\left(\alpha^{j}\right)=\sum_{n=-\infty, n \neq 0}^{+\infty} \frac{e^{i n B \alpha^{j}}}{i n B} .
$$

Taking curl of $S\left(\alpha^{j}\right) \nabla\left(r{\overline{V_{\theta}}}^{j}\right)$

$$
\begin{aligned}
\nabla \times\left[S\left(\alpha^{j}\right) \nabla\left(r \bar{V}_{\theta}^{j}\right)\right] & \left.=\nabla S\left(\alpha^{j}\right) \times \nabla\left(r{\overline{V_{\theta}}}^{j}\right)+S\left(\alpha^{j}\right) \nabla^{2}\left(r{\overline{V_{\theta}}}^{j}\right)\right) \\
& =S^{\prime}\left(\alpha^{j}\right) \nabla \alpha^{j} \times \nabla\left(r \overline{V_{\theta}}\right)+0 \\
& =-\nabla\left(r{\overline{V_{\theta}}}^{j}\right) \times \nabla \alpha^{j} S^{\prime}\left(\alpha^{j}\right)
\end{aligned}
$$

Using Eq. 6.60 and 6.58 leads to

$$
\tilde{\boldsymbol{v}}^{j}=\nabla \tilde{\phi}^{j}-S\left(\alpha^{j}\right) \nabla\left(r \overline{V_{\theta}}\right)^{j}
$$

Thus,

$$
\overline{\boldsymbol{V}}^{j}=\nabla \bar{\phi}^{j}+\left(r \overline{V_{\theta}}\right)^{j} \nabla \alpha^{j}
$$

The continuity equation Eq. 6.33 can be written as

$$
\nabla \cdot \tilde{\boldsymbol{v}}=-\tilde{\boldsymbol{v}} \cdot \nabla \ln \bar{\rho}
$$


Substituting Eq. 6.61 into 6.63 gives

$$
\nabla^{2} \tilde{\phi}^{j}=S\left(\alpha^{j}\right) \nabla^{2}\left(r V_{\theta}\right)^{j}-S^{\prime}\left(\alpha^{j}\right)\left(r V_{\theta}\right)^{j} \nabla \alpha^{j}-\tilde{\boldsymbol{v}}^{j} \cdot \ln \bar{\rho}^{j}
$$

where the first two terms on the RHS are zeros outside the blade rows. We can express the potential $\tilde{\phi}^{j}$ using the Fourier series since it is periodic in the pitchwise direction,

$$
\tilde{\phi}^{j}(r, \theta, z)=\sum_{n=-\infty}^{\infty} \tilde{\phi}_{n}(r, z) e^{i n B \theta} .
$$

Inserting Eq. 6.65 into 6.64 yields

$$
\begin{aligned}
\nabla^{2} \tilde{\phi}(r, \theta, z) & =\sum_{n=-\infty}^{\infty}\left[\frac{1}{r} \frac{\partial}{\partial r}\left(r \frac{\partial \tilde{\phi}_{n}}{\partial r}\right)+\frac{\partial^{2} \tilde{\phi}_{n}}{\partial z^{2}}-\frac{\tilde{\phi}_{n}}{r^{2} n^{2} B^{2}}\right] e^{i n B \theta} \\
& =\sum_{n=-\infty}^{\infty}\left[\frac{1}{r} \frac{\partial \tilde{\phi}_{n}}{\partial r}+\frac{\partial^{2} \tilde{\phi}_{n}}{\partial r^{2}}+\frac{\partial^{2} \tilde{\phi}_{n}}{\partial z^{2}}-\frac{\tilde{\phi}_{n}}{r^{2} n^{2} B^{2}}\right] e^{i n B \theta}
\end{aligned}
$$

where the Laplacian $\nabla^{2}$ in the cylindrical coordinates is

$$
\nabla^{2}=\frac{1}{r} \frac{\partial}{\partial r}\left(r \frac{\partial}{\partial r}\right)+\frac{1}{r^{2}} \frac{\partial^{2}}{\partial \theta^{2}}+\frac{\partial^{2}}{\partial z^{2}}
$$

The nth harmonic of the potential function for the periodic flow is

$$
\begin{aligned}
& \left\{\frac{\partial^{2} \phi_{n}}{\partial r^{2}}+\frac{1}{r} \frac{\partial \phi_{n}}{\partial r}+\frac{\partial^{2} \phi_{n}}{\partial z^{2}}+\frac{\partial \phi_{n}}{\partial z} \frac{\partial(\ln \bar{\rho})}{\partial z}+\frac{\partial \phi_{n}}{\partial r} \frac{\partial(\ln \bar{\rho})}{\partial r}-\frac{n^{2} B^{2}}{r^{2}} \phi_{n}\right\}^{j} \\
= & \left\{\frac{e^{-i n B f(r, z)}}{i n B}\left[\nabla^{2} r \overline{V_{\theta}}+\frac{\partial\left(r \overline{V_{\theta}}\right)}{\partial r} \frac{\partial(\ln \bar{\rho})}{\partial r}+\frac{\partial\left(r \overline{V_{\theta}}\right)}{\partial z} \frac{\partial(\ln \bar{\rho})}{\partial z}\right]\right. \\
& \left.-e^{-i n B f(r, z)}\left[\frac{\partial f}{\partial r} \frac{\partial\left(r \overline{V_{\theta}}\right)}{\partial r}+\frac{\partial f}{\partial z} \frac{\partial\left(r \overline{V_{\theta}}\right)}{\partial z}\right]\right\}^{j}
\end{aligned}
$$

In order to solve the unknown Fourier coefficients in the above potential flow equation, the physical boundary conditions need to be imposed:

- End wall boundary conditions at hub and shroud. On the hub and shroud wall, the velocity components normal to the hub and 
shroud contour must vanish. Thus,

$$
\begin{gathered}
\tilde{\boldsymbol{v}} \cdot \boldsymbol{n}_{h}=0, \\
\tilde{\boldsymbol{v}} \cdot \boldsymbol{n}_{s}=0,
\end{gathered}
$$

in which $\boldsymbol{n}_{h}$ and $\boldsymbol{n}_{s}$ are the normal vectors to the hub and shroud. Inserting Eq. 6.69 and 6.70 into Eq. 6.64

$$
\begin{aligned}
\frac{\partial \phi_{n}^{j}}{\partial n_{h}} & =\frac{\partial\left(r V_{\theta}\right)^{j}}{\partial n_{h}} \frac{e^{-i n B f^{j}(r, z)}}{i n B}, \\
\frac{\partial \phi_{n}^{j}}{\partial n_{s}} & =\frac{\partial\left(r V_{\theta}\right)^{j}}{\partial n_{s}} \frac{e^{-i n B f^{j}(r, z)}}{i n B},
\end{aligned}
$$

- Uniform flow at the far upstream and downstream. The flow is assumed to be perfectly uniform at far upstream and downstreams, i.e., there is only axial velocity at far upstream and only radial velocity at the downstream. This can be realised by imposing,

$$
\phi_{n}^{j}=0
$$

Thus, for each of the harmonics of the periodic flow Eq. 6.68 can be solved subject to the Neumann boundary conditions using Eq. 6.71, 6.72, and Dirichlet boundary condition Eq. 6.73.

- Kutta-Joukowski condition at the trailing edge. At the blade trailing edge, Kutta-Joukowski condition imposes zero tangential pressure loading, i.e., $p^{-}=p^{+}$. Recall Eq. 6.19, the Kutta-Jowkowski condition requires that

$$
W_{m b l}^{j} \frac{\partial\left(r \overline{V_{\theta}}\right)^{j}}{\partial m^{j}}=0
$$

which holds true at the blade trailing edge. A similar condition can also be imposed at the leading edge. If $W_{m b l}^{j} \frac{\partial\left(r \overline{V_{\theta}}\right)^{j}}{\partial m^{j}}$ is set to be zero at the leading edge, it means that the blade row designed is precisely parallel to the local velocity vector, i.e., zero incidence angle at the leading 
edge. It is however not necessary to maintain the zero incidence angle at the leading edge. As a matter of fact, the leading edge loading has complete freedom in the current inverse method for optimising a blade design.

\subsubsection{Calculation of the velocity - exact method}

\section{The modified continuity equation}

So far we have described a method to calculate the density and an approximate to calculate the mean and periodic velocity field based on the assumption that the pithwise variation of density is completely ignored. This approximation can reduce the complexity of numerically evaluating the three dimensional flow field at a cost of accuracy. An exact method will be discussed below when the density variations are considered at all three dimensions.

Let us first recall the continuity equation 6.33, the tangential average of this equation must take the form

$$
\nabla \cdot \overline{\boldsymbol{W}}=-\overline{\boldsymbol{W} \cdot \nabla \ln \rho}
$$

which cannot be directly used to define the stream function as used in Eq. 6.39 neither can a governing equation for the mean flow be obtained. Let us define an artificially modified density $\rho_{a m}$ holding the equation

$$
\nabla \cdot \rho_{a m} \overline{\boldsymbol{W}}=0
$$

to be valid. $\rho_{a m}$ can be found through the equation below

$$
\overline{\boldsymbol{W}} \cdot \nabla \ln \rho_{a m}=\overline{\boldsymbol{W} \cdot \nabla \ln \rho}
$$

which can be interpreted as: the tangential average of the dot product between the relative velocity and gradient of the density's natural logarithm is equivalent to the dot product between the mean of the relative velocity and gradient of an artificially modified density's natural logarithm. Eq. 6.76 
then allows the same stream function treatment used in the last subsection for the mean flow field calculations.

2. The surface vorticity method and Clebsch formulation for periodic flow

Using the artificially modified density the continuity equation 6.63 for the periodic flow is recast into

$$
\begin{aligned}
\nabla \cdot \tilde{\boldsymbol{v}} & =-\nabla \cdot \overline{\boldsymbol{W}}-\overline{\boldsymbol{W}} \nabla \ln \rho-\tilde{\boldsymbol{v}} \nabla \ln \rho \\
& =-\nabla \cdot \overline{\boldsymbol{W}}-\boldsymbol{W} \cdot \nabla \ln \rho \\
& =-\overline{\boldsymbol{W} \cdot \nabla \ln \rho}-\boldsymbol{W} \cdot \nabla \ln \rho
\end{aligned}
$$

Consequently, Eq. 6.64 is changed to

$$
\begin{aligned}
\nabla^{2} \tilde{\phi}^{j}= & S\left(\alpha^{j}\right) \nabla^{2}\left(r V_{\theta}\right)^{j}-S^{\prime}\left(\alpha^{j}\right)\left(r V_{\theta}\right)^{j} \nabla \alpha^{j}-\tilde{\boldsymbol{v}}^{j} \cdot \ln \bar{\rho}^{j} \\
& +\overline{\boldsymbol{W}^{j} \cdot \nabla \ln \rho^{j}}-\boldsymbol{W}^{j} \cdot \nabla \ln \rho^{j}
\end{aligned}
$$

which is very similar Eq. 6.64 everywhere except for the third and fourth terms included due to the new strategy of writing the continuity equation. Zangeneh [1991] has explained that Eq. 6.79 can be solved by using the inverse discrete Fourier transform, which is,

$$
\phi(r, \theta, z)^{j}=\sum_{n=-N / 2}^{N / 2-1} \phi_{n}(r, z)^{j} e^{i k_{n} \theta_{q}}
$$

where $\theta_{q}=q \Delta \theta, q$ is non-negative integer. $\theta_{q}$ represent the tangential coordinates of a set of points on a uniformly spaced grid in the tangential directon. The set $k_{n}$ is defined as,

$$
k_{n}=\frac{2 \pi n}{N \Delta \theta}
$$

for $n=-\frac{N}{2}, \cdots, 0, \cdots, \frac{N}{2}-1$, and it should be chosen to eliminate the aliasing effects.

Substituting Eq. 6.80 and 6.81 into Eq. 6.79 the governing equation for the 
periodic flow is obtained as following,

$$
\begin{aligned}
& \frac{\partial^{2} \phi_{n}}{\partial r^{2}}+\frac{1}{r} \frac{\partial \phi_{n}}{\partial r}+\frac{\partial^{2} \phi_{n}}{\partial z^{2}}+\frac{\partial \phi_{n}}{\partial z} \frac{\partial(\ln \bar{\rho})}{\partial z}-\frac{n^{2} B^{2}}{r^{2}} \phi_{n}+R_{n}(r, z) \\
= & \frac{e^{-i n B f(r, z)}}{i n B} \nabla^{2} r \overline{V_{\theta}}-e^{-i n B f(r, z)}\left[\frac{\partial f}{\partial r} \frac{\partial\left(r \overline{V_{\theta}}\right)}{\partial r}+\frac{\partial f}{\partial z} \frac{\partial\left(r \overline{V_{\theta}}\right)}{\partial z}\right],
\end{aligned}
$$

where

$$
R_{n}(r, z)=\frac{1}{N} \sum_{q=1}^{N}(\boldsymbol{W} \cdot \nabla \ln \rho) e^{-\frac{2 \pi q n}{N}} .
$$

When $n=0$, Eq. 6.82 becomes the Clebsch formulation of the mean flow continuity equation and thus it needs to be neglected in the solutions of the periodic flow field. Since the potential function of the periodic flow is real, its Fourier transform has the following property with its conjugate,

$$
\left(\phi_{-n}\right)^{j}=\left(\phi_{n}^{* *}\right)^{j}
$$

where $* *$ denotes the complex conjugate. Eq. 6.82 is then only needed to solved for half of the frequency spectrum $k_{n}$ defined in Eq. 6.81.The boundary conditions needed for solving Eq. 6.82 are the same as what has been described in the last subsection, i.e., 1) zero normal velocity at hub and shroud wall (Neumann condition); 2) purely axial periodic flow and radial periodic flow at far upstream and downstream respectively (Dirichlet condition); 3) Kutta-Joukowski boundary condition (no pressure different at the blade TE).

\subsubsection{Blade shape calculation}

In the last subsection the detailed formulations for determining the flow field are presented. Once the flow field information is known, it is possible to compute the blade shape based on the following boundary condition,

$$
\boldsymbol{W}_{b l}^{j} \cdot \nabla \alpha^{j}=0
$$


which means the blade shape needs to be aligned to the velocity vector, and

$$
\boldsymbol{W}_{b l}^{j}=\left(\frac{W^{+}-W^{-}}{2}\right)^{j}
$$

where $W^{+}$and $W^{-}$are velocities of the flow on the upper and lower surface respectively. Inserting Eq. 6.86 into 6.85 yields,

$$
\bar{V}_{z}^{j} \frac{\partial f^{j}}{\partial z}+\bar{V}_{r}^{j} \frac{\partial f^{j}}{\partial r}=\sum_{k=1}^{2} \frac{\left(r \overline{V_{\theta}}\right)^{k}}{r^{2}}-\omega+\left(\tilde{\boldsymbol{v}}_{b l}^{j} \cdot \nabla \alpha^{j}\right),
$$

When the superscripts are equal to 1, then Eq. 6.87 pertains to the full bade, and when it is equal to 2, Eq. 6.87 pertains to the splitter blade. The above equation is a first order hyperbolic partial differential equation and it can be solved by integrating it along the meridional projection of the streamlines on the blade surface. It cannot be solved without specifying the initial value for $f$. This value shall be called the stacking condition of the blade. In the current method the values of the blade wrap angle $f$ at the blade trailing edge are given as inputs.

Eq. 6.42, 6.68, 6.82 and 6.87 are solved using finite difference method in a body fitted coordinate $(\xi-\eta)$ tranformed from the meridional frame $r-z$. The detailed numerical algorithms can be found in section 2.5 and 2.6 of the work by Zangeneh [1991].

\subsection{Parametrisation of the blade loading}

In the last three subsections a great deal of mathematical formulations and physical interpretation of the inverse method's flow solver and blade shape generation algorithm have been discussed. We have emphasised that the primary feature of the current inverse method is its prescription of the blade lading. This quantity directly affects the fluid dynamics in the blade channel, resulting in different exit flow performances. Therefore, controlling the blade loading can help to control the performances of a turbomachine. In this subsection, we will present the way in which the blade loading is parametrically defined. 


\subsection{1 $r V_{\theta}^{*}$ at LE and TE}

First we introduce $r V_{\theta}^{*}$, which is a non-dimensionalised parameter and defined by

$$
r V_{\theta}^{*}=\frac{r \overline{V_{\theta}}}{r_{t i p} U_{t i p}},
$$

in which $r_{t i p}$ and $U_{\text {tip }}$ are the impeller's radius and tangential speed at the trailing edge. If we recall Eq. 6.1, we know that this quantity is the specific Euler work of a turbomachine. The average change of this quantity from the inlet to outlet determines how much energy the flow receives/gives to the impeller. It is possible to define $r V_{\theta}^{*}$ with some variations from hub to shroud at both inlet and outlet. We also need to remind ourselves that $r V_{\theta}^{*}$ has been divided into two parts for the full and splitter blades separately. Thus, it is also possible to specify the ratio between $\left(r V_{\theta}^{*}\right)^{1}$ and $\left(r V_{\theta}^{*}\right)^{2}$. Again, here the superscript 1 and 2 pertain to the full and splitter blade respectively. Let us define a work ratio $\kappa$ that controls the splitter's contribution, as is given below,

$$
\kappa=\frac{\left(r V_{\theta}^{*}\right)^{2}}{r V_{\theta}^{*}}
$$

where $r V_{\theta}^{*}=\sum_{j=1}^{2}\left(r V_{\theta}^{*}\right)^{j}$. This is a very useful parameter that can help to divide the work contributed from the full and splitter blade which in turn results in many different flow behaviours in the blade channel. Thus, this work ratio $\kappa$ can also be treated as an optimisation parameter.

To summarise:

- At the leading edge: $r V_{\theta}^{*}$ is a given constant. It needs to be zero everywhere from hub to shroud for a free vortex design.

- At the trailing edge: $r V_{\theta}^{*}$ is a given constant. It needs to remain constant from hub to shroud for a free vortex design but is not essential for best overall performance of a compressor. In practice it is also common to introduce some variations to $r V_{\theta}^{*}$ from hub to shroud section to adjust the exit flow's uniformity and reduce tip leakage flow.

- The splitter work ratio: $\kappa$ needs to be properly defined at both hub and 
shroud so that feasible solutions can be found. Too large or small value of $\kappa$ will result in non-realistic blade geometry or convergence difficulties for the solver. It is common practice to initially define the $\kappa$ to be close to meridional length ratio between the splitter and full blade.

\subsection{2 $\partial r V_{\theta}^{*} / \partial m^{*}$ from the inlet to outlet}

Now let us recall the blade loading equation for the compressible flow

$$
\Delta h^{j}=\left(h^{+}-h^{-}\right)^{j}=\frac{2 \pi}{B}\left(W_{m b l} \frac{\partial\left(r V_{\theta}^{*}\right)}{\partial m^{*}}\right)^{j}
$$

in which $m^{*}$ is the normamlised meridional distance from impeller leading to trailing edge. It is clear that $\Delta h$ is determined by the values of $W_{m b l}$ and $\partial\left(r V_{\theta}^{*}\right) / \partial m^{*}$. As has been pointed by Borges [1990a] $W_{m b l}$ should vary smoothly from the inlet to outlet in a machine well designed. So, the blade loading will roughly vary in a way that follows the distribution of $\partial\left(r V_{\theta}^{*}\right) / \partial m^{*}$ from the inlet to outlet along the blade streamlines. The problem is now reduced to the specification of $\partial\left(r V_{\theta}^{*}\right) / \partial m^{*}$ along the streamlines of full and splitter blade from the inlet to outlet. Let us assume a frame made of $\partial\left(r V_{\theta}^{*}\right) / \partial m^{*}$ and $m^{*}$. As has been discussed in the last section, this quantity at the leading $(m *=0)$ and trailing $(m *=1)$ edges is subject to imposed boundary conditions.

- $m^{*}=0$. At the inlet, the blade loading will largely determine the blade row's incidence angle. If $\partial\left(r V_{\theta}^{*}\right) / \partial m^{*}$ is set to be zero, then the blade row is perfectly aligned with inlet flow velocity. However, it is not necessary to be zero for the optimal performances. Adjusting $\partial\left(r V_{\theta}^{*}\right) / \partial m^{*}$ at the inlet is a very useful way for controlling the peak efficiency point and operating range of a turbomachine.

- $m^{*}=1$. At the outlet, the blade loading is constrained to be zero to satisfy the Kutta-Joukowski condition, i.e., $\partial\left(r V_{\theta}^{*}\right) / \partial m^{*}$ is always zero at the impeller exit.

- $0<m^{*}<1$. Between the inlet and outlet there can be infinite ways constructing the $\partial\left(r V_{\theta}^{*}\right) / \partial m^{*}$, but the rule of thumb is to represent as many 


\section{MATHEMATICAL FORMULATIONS OF THE INVERSE}

blade loadings as possible with as few parameters as possible. Since the optimisation in inverse design approach is not the geometric parameters optimisation but the aerodynamic parameters optimisation, the fewer parameters used to define the blade loading, the less effort required. For example, Tjokroaminata [1992]; Yang [1991] used a biharmonic solver to define the blade loading distribution as a polynomial curve, which is not every easy to implement. In the current study an alternative method is used for simplicity. The blade loading curve consists of three segments connecting from the inlet to outlet in the meridional direction. At two ends two parabolic curves are used and a linear curve is used to connect the two parabolic curves. This arrangement requires four variables to define a complete blade loading curve, which are explained below:

- DRVT. It is the parameter of the normalised $r V_{\theta}^{*}$ at the inlet where $m^{*}=0$;

- NC. It is the parameter of normalised $m^{*}$ where the first parabolic curve ends;

- ND. It is the parameter of normalised $m^{*}$ where the second parabolic curve begins, and it must be greater than $\mathrm{NC}$;

- Slope. It is the slope of a linear curve that connects the parabolic curves at NC and ND.

Both of the full and splitter blade loading curve are defined in this same manner, and it is possible to define the blade loading curves at all hub, midspan, and shroud section, or for simplicity, defining at hub and shroud is also sufficient. Figure 6.2 is an example of the blade loading curves constructed in such a three segment way. Since each blade loading curve is constructed using four different parameters, the total number of parameters controlling $\partial\left(r V_{\theta}^{*}\right) / \partial m^{*}$ including three sections for both full and splitter blades is 24 . There are four other parameters controlling $r V_{\theta}^{*}$ distribution, which are the values of $r V_{\theta}^{*}$ at the impeller exit's hub and shroud, and values of $\kappa$ at the impeller exit's hub and shroud. Thus, the total aerodynamic field is controlled by 28 parameters. Using these parameters listed here, we 


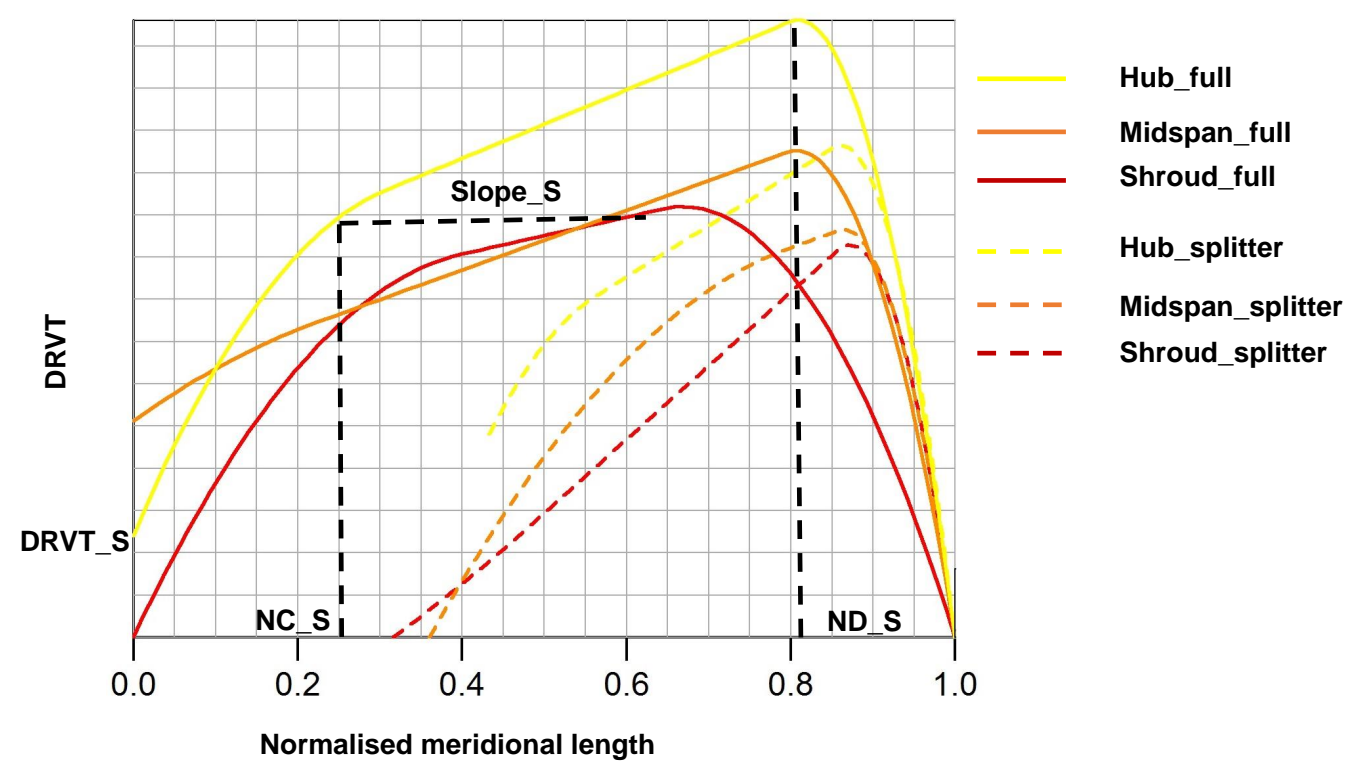

Figure 6.2: Blade loading parametrisation curve

can convert the geometric parametrisation problem of the conventional/direct design approach into the aerodynamic or hydrodynamic (when flow is a liquid rather than a gas) parametrisation. It is not difficult to understand that this inverse method has more correlation with turbomachine's performances. However, it is rarely needed to perform the optimisation using all the 28 parameters listed here. A great deal of experience has already been accumulated on how to set the blade loading parameters for the optimal performances.

\subsection{Summary}

Quite thorough explanations of the key mathematical formations for the inverse design theory have been presented in this chapter. Starting with the fundamental turbomachinery Euler equation, a correlation between the blade surface circulation and the loading on each point of the blade has been derived using the Kutta-Joukowski lift force theory. In the previous literatures (Hawthorne et al. [1984]; Tan et al. [1984]), no such step-by-step derivations were given, which has caused difficulty for readers to fully comprehend the essence of this elegant design 


\section{MATHEMATICAL FORMULATIONS OF THE INVERSE}

DESIGN METHOD

theory. It is therefore decided to present the derivation here in a rigours mathematical way with physical interpretations. In addition to that, it has shown the difference between the blade loading prescription for the incompressible and compressible flow conditions. Essentially, it is the static pressure difference between upper and low surface are prescribed for the incompressible flow, whereas the enthalpy jump must be used for the compressible flow. In the work by Zangeneh [1991], a different approach to derive the enthalpy jump was used. However, the method used in this chapter is more intuitive and easier for understanding.

Following the complete derivation of key blade loading equation, the key formulations for the flow solver were presented. This part of the work was also fully explained in the work by Zangeneh [1988] and Zangeneh [1991].

In the end, the parametrisation of the blade loading was explained. The so-called three-segment method is quite robust and has achieved a number of successful designs. A latest example is a redesign of a low pressure ratio compressor for electric supercharger used in a heavily downsized engine. Wang et al. [2017] has shown by both CFD predictions and test data that the redesigned compressor has significant advantages in terms of pressure ratio, choke/surge margin and efficiency. 
"Design is not just what it looks like and feels like.

Design is how it works."

Steve Jobs (1955 - 2011)

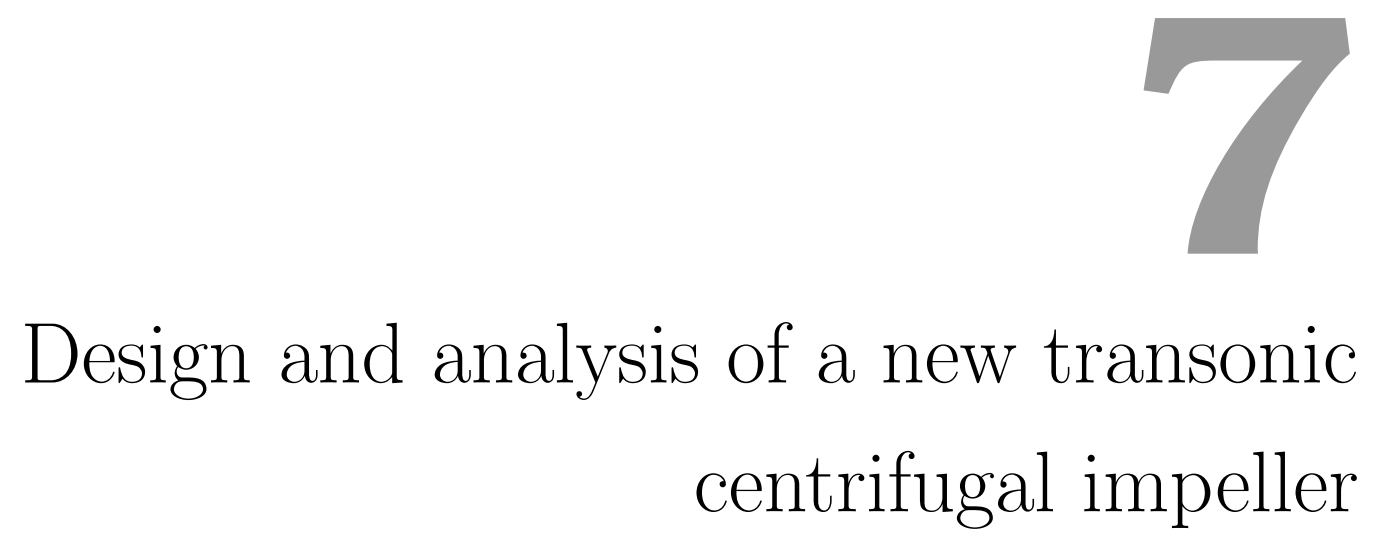

In this chapter the design and analysis for a new transonic impeller will be presented. We shall begin with the analysis of the existing compressor used by Napier Turbochargers. Its aerodynamic performances will be predicted by CFX and compared with the test data to validate the CFD model used. The redesigned procedures in TURBOdesign1 shall then be discussed in detail, and the redesigned impeller will be simulated in the same way as the baseline design to ensure the comparisons are valid. In addition, the FEA modelling will be applied to investigate both impellers' structure integrity.

Figure 7.1 shows a schematic of the overall design process. As can be seen, the FEA analysis is first carried out after a design is generated by TD1 to confirm the mechanical feasibility. If a design cannot pass the mechanical criterion, further simulations are not allowed. The information will be fed back to the design block to get a new design. Once the FEA analysis confirms a design's mechanical 


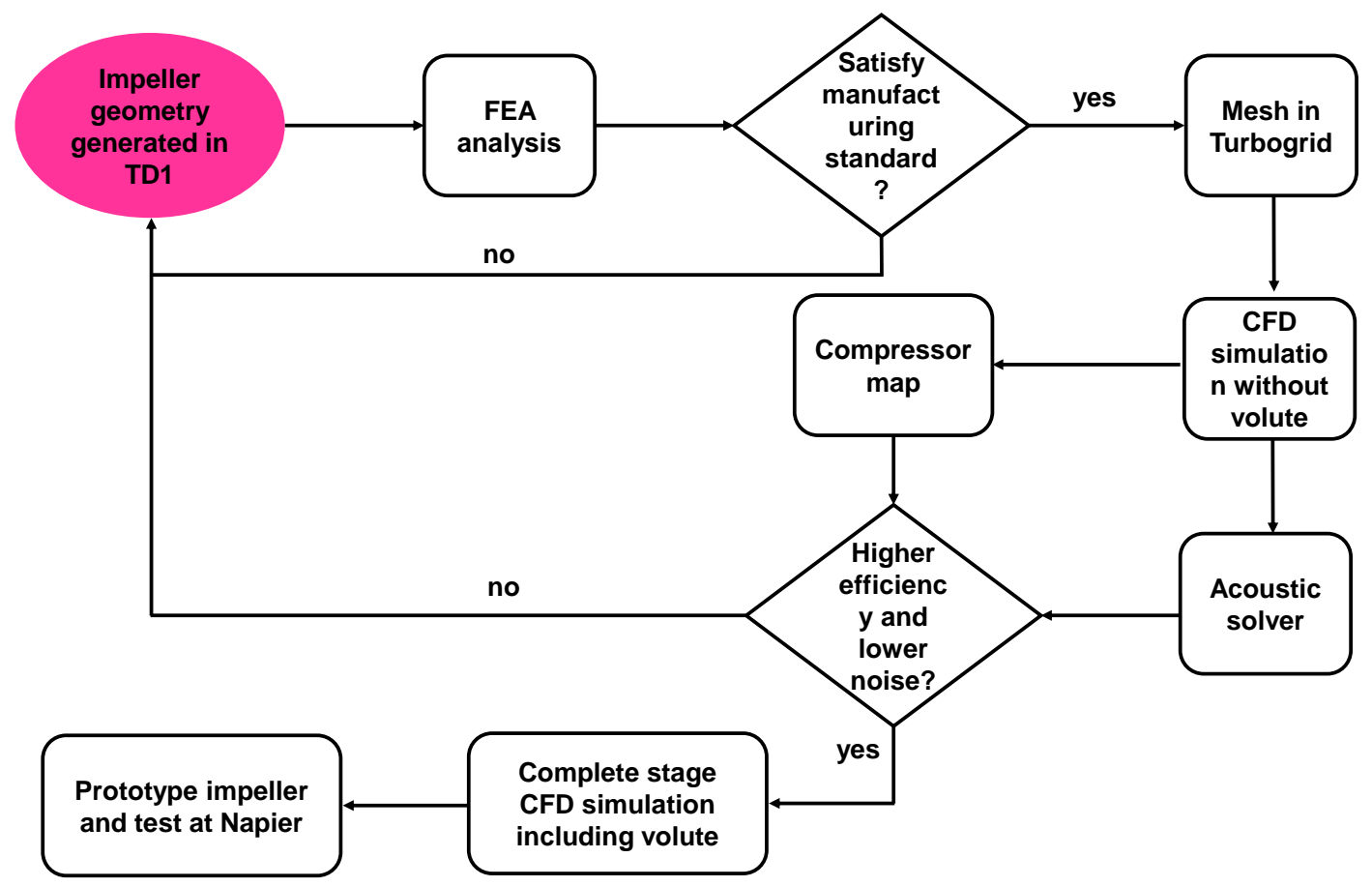

Figure 7.1: Overall design flow chart for the new impeller

feasibility, it will be meshed and simulated using CFX with no volute included. The compressor characteristic map can then be generated and compared with the baseline's. At the same time the fluid quantities calculated will be imported to the acoustic solver for the noise level evaluations. Once a design with both higher efficiency and lower noise is found, the complete stage will be used for CFD simulations. The final design will be prototyped and tested at Napier's test stand.

\subsection{Analysis of the existing compressor stage}

The existing compressor stage comprises a ported shroud, an impeller wheel, a vaned diffuser wheel, and a volute. It is a state-of-the-art product of Napier Turbocharger Ltd, whose application mainly lies in large marine ships. In the present study, only the impeller wheel was redesigned and optimised. A complete redesign and evaluation of all components is beyond the purpose of this thesis. 


\subsubsection{Geometry details of baseline impeller}

The baseline wheel has a common full-splitter blade arrangement used in turbochargers for the wide operating range and high performance. Its important geometric parameters are summarised in Table 7.1. The meridional housing of this impeller is given in Fig. 7.2 and its $3 \mathrm{D}$ view is given in Fig. 7.3.

Table 7.1: Baseline impeller parameters

\begin{tabular}{|l|l|}
\hline Shaft speed & $17 \mathrm{k}-32.5 \mathrm{k} \mathrm{rpm}$ \\
\hline Blade count full/splitter & $11 / 11$ \\
\hline Full LE hub radius & $40.81 \mathrm{~mm}$ \\
\hline Full LE shroud radius & $118.28 \mathrm{~mm}$ \\
\hline Diffuser inclination against radial & $13 \mathrm{deg}$ \\
\hline Tip clearance & $0.504 \mathrm{~mm}$ at inlet to $0.557 \mathrm{~mm}$ at exit \\
\hline Diffuser inlet radius $\left(r_{3}\right)$ & $210.51 \mathrm{~mm}$ \\
\hline Diffuser outlet radius $\left(r_{4}\right)$ & $283.65 \mathrm{~mm}$ \\
\hline
\end{tabular}

\subsubsection{CFD analysis for the existing compressor stage}

In this subsection, the CFD simulations are used to predict the baseline compressor stage's performances and the predictions will be validated against the test data.

\subsubsection{Grid independence study}

First, a grid independence was carried out to get the minimum mesh size needed for impeller and diffuser. It is assumed that the incoming flow is normal to the inlet surface and total temperature and pressure used are $298 \mathrm{~K}$ and $101.325 \mathrm{kPa}$. Only one (out of 11) impeller and one (out of 19) diffuser channel were used. The interface between the rotor and stator domain was set to be stage model, which performs a circumferential averaging of the fluxes on the interface. Thanks to the same impeller exit and diffuser inlet width, the pitch ratio between rotating and stationary domain is $11 / 19$. Their interface plane is radially off-set by $3 \mathrm{~mm}$ from impeller's trailing edge. At the outlet different static pressures were used first until the compressor started stall, the mass flow boundary conditions were then 


\section{DESIGN AND ANALYSIS OF A NEW TRANSONIC CENTRIFUGAL IMPELLER}

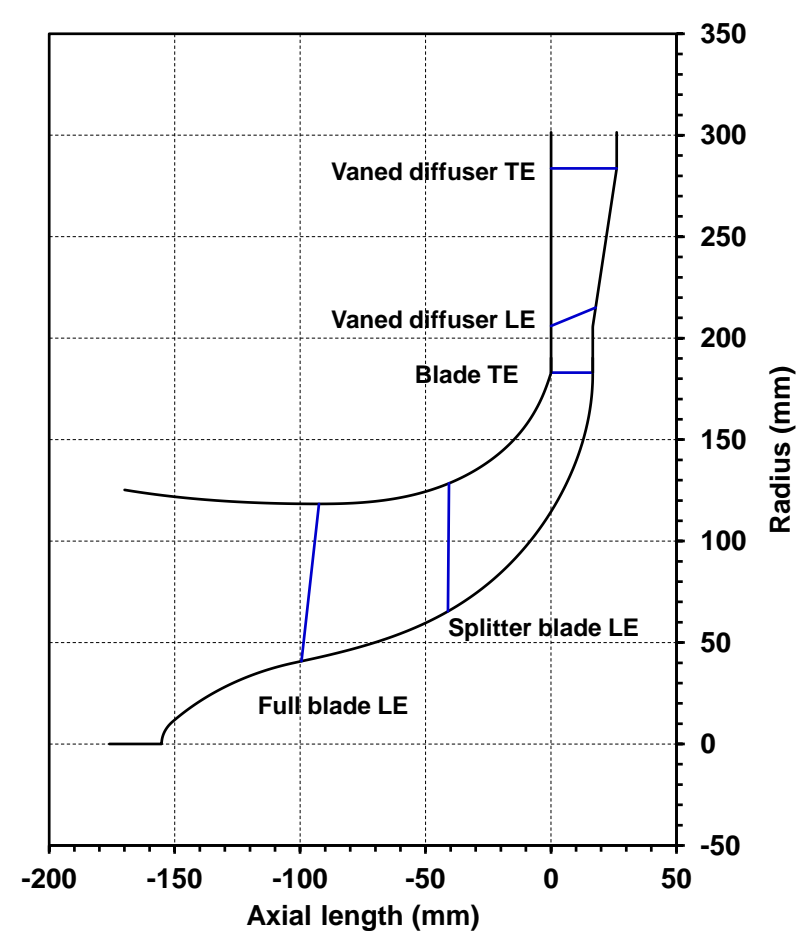

Figure 7.2: Meridional contour of the baseline impeller and diffuser

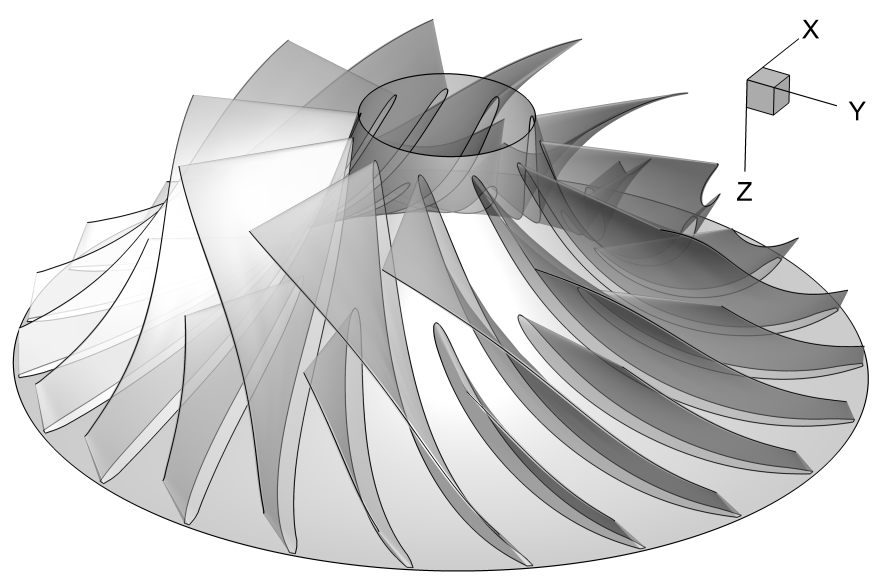

Figure 7.3: 3D view of the baseline impeller wheel 


\section{DESIGN AND ANALYSIS OF A NEW TRANSONIC CENTRIFUGAL IMPELLER}

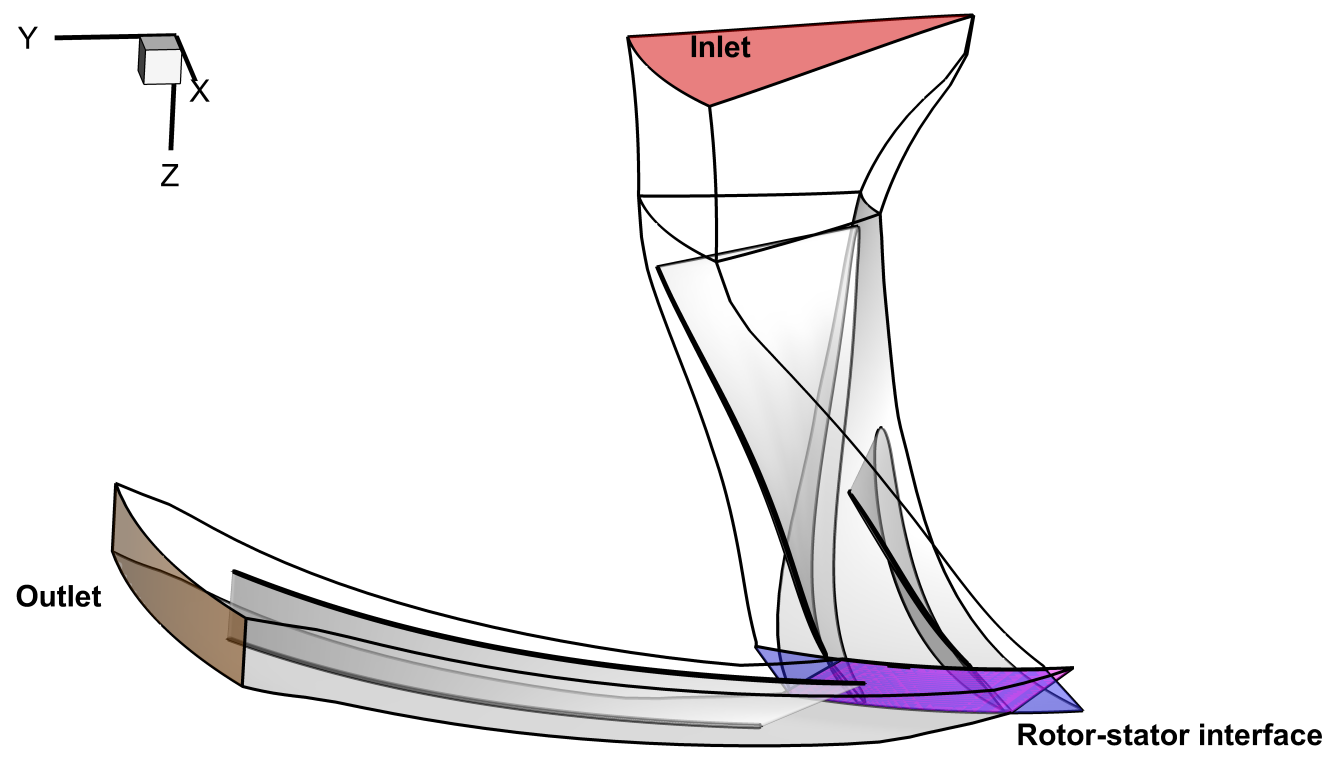

Figure 7.4: Impeller-diffuser set up in CFD

Table 7.2: Three different meshes used for the mesh-independence study

\begin{tabular}{llll}
\hline & Fine & Medium & Coarse \\
\hline Impeller & $1748 \mathrm{k}$ & $992 \mathrm{k}$ & $432 \mathrm{k}$ \\
Diffuser & $401 \mathrm{k}$ & $231 \mathrm{k}$ & $105 \mathrm{k}$ \\
\hline
\end{tabular}

selected. The mass flow rate was reduced gradually until no further convergence was possible. As to the turbulence modelling, the shear stress transport (SST) model was used. Structured meshes were created for the impeller and diffuser using TurboGrid, which generated three different meshes, termed as, "coarse", "medium" and "fine" for calculations. Table 7.2 presents the grid numbers for the impeller and diffuser respectively. In Fig. 7.5 are compared the compressor characteristics predicted using these three different mesh schemes at speedline 1800 (about 30krpm), all of which are able to ensure $\mathrm{Y}+$ less than 5 everywhere except the cut-off trailing edge. The mass flows are normalised values, which are divided by the design point mass flow. It can seen that all three meshes predict very similarly choke margin, however, as the mass flow reduces, the pressure ratio and efficiency predicted by coarse mesh are obviously lower than those predicted by medium and fine mesh simulations. On the other hand the medium and fine 


\section{DESIGN AND ANALYSIS OF A NEW TRANSONIC CENTRIFUGAL IMPELLER}
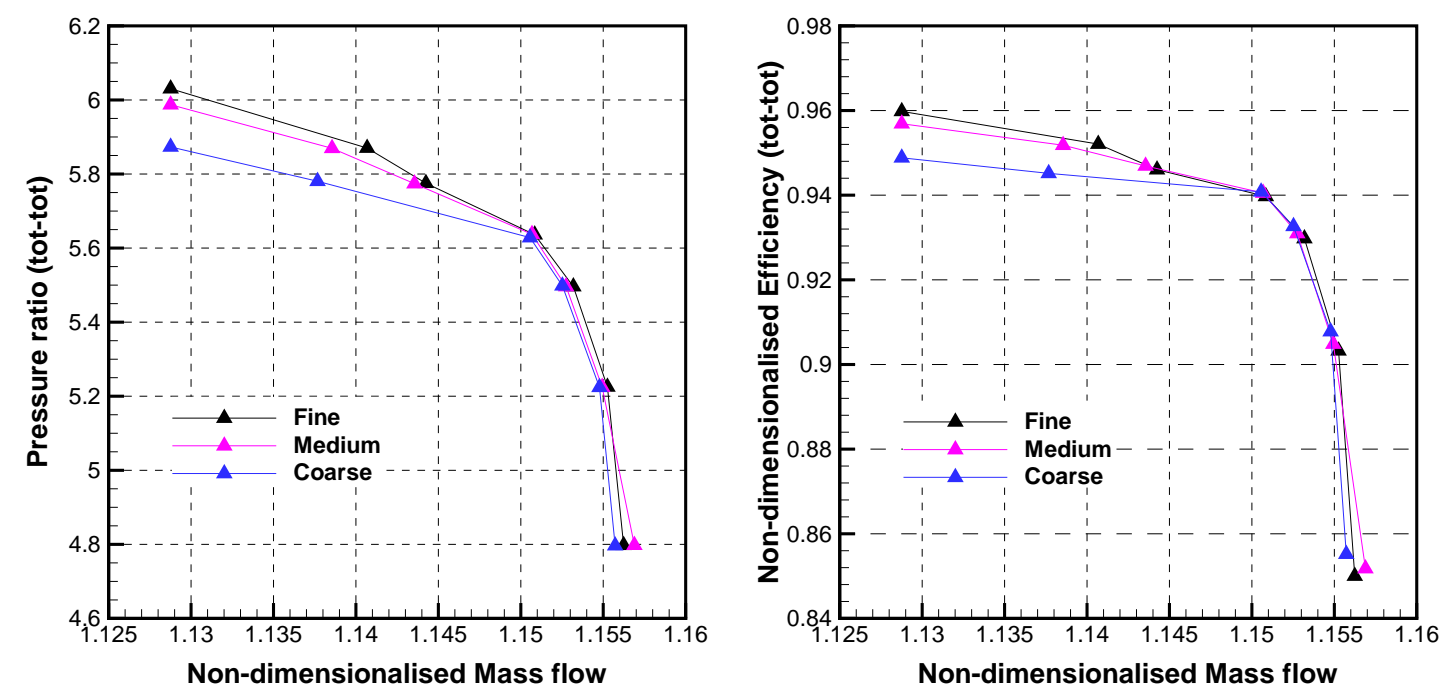

Figure 7.5: CFD predictions of the compressor characteristics under three meshes in Table 7.2 at speedline 1800 (about 30krpm)

meshes give fairly close results until the surge point. It is therefore decided to use the medium-sized mesh as the benchmark for all future simulations.

\subsubsection{Validation with test results}

The complete compressor stage simulations including impeller, diffuser and volute were performed at four different speedlines. As Fig. 7.6 shows, the CFD set up for the compressor stage includes an intake connected to a single blade channel, followed by a single vaned diffuser channel and a complete volute. The two interfaces, between the rotor-stator and stator-volute respectively, are both modelled by "stage model" (also known as mixing plane) in CFX. The inlet and outlet boundary conditions were set up in same way as previously mentioned.

Figures 7.7 and 7.8 show the comparisons of the predicted pressure ratio and efficiency versus the mass flow characteristics at 4 different speedlines $N_{s}$ $\left(N_{s}=\omega / \sqrt{T}\right.$, in which $\omega$ is the rotational speed in $\operatorname{rpm}$ and $T$ is reference temperature at the inlet). In general there is good agreement between the CFD and experimental results except that there is some underestimation of the choke margin at speedline 1600, and the reason is still not clear. It should be noted that the predicted stall margins for speedlines 1300, 1600, and 1800 are less 


\section{DESIGN AND ANALYSIS OF A NEW TRANSONIC CENTRIFUGAL IMPELLER}

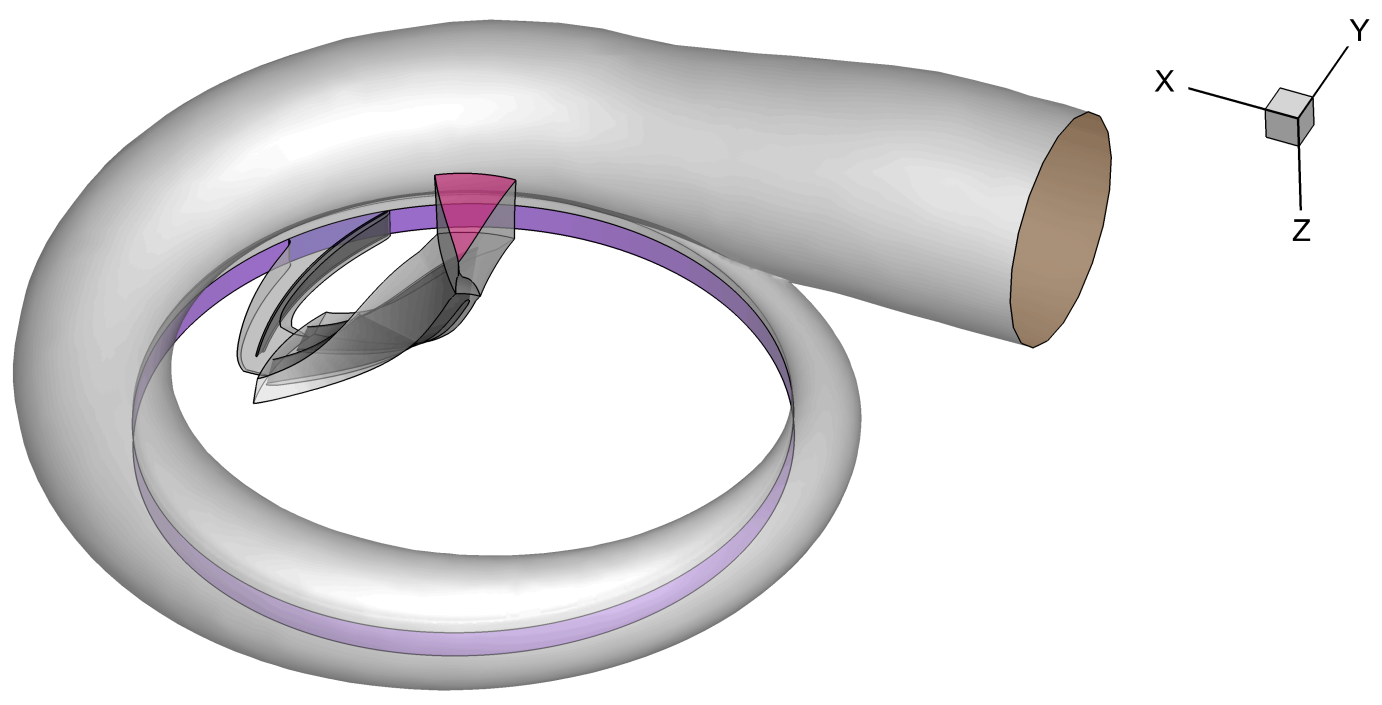

Figure 7.6: CFD set up for the compressor stage simulation

than the measurements, which is likely due to the ported shroud included in the test but not in the CFD calculations. Since the complete 3D CAD geometry of the ported shroud was not available, we could not include it in the simulations. The predicted stall margin at speedline 1000 is very close to the experimental one, which conforms to the usual knowledge that the ported shroud can only effectively work for high pressure ratio conditions. The CFD calculated efficiencies at different speedlines are greater than the test results, which can result from:

- friction and non-adiabatic losses that are not modelled in CFD;

- losses due to the reverse flow in the ported shroud;

- the "stage model" idealised the flow dynamics across the interface and underestimated the mixing losses;

- the temperatures measured by the probes at the discharging duct could not represent the mass averaged temperatures at the outlet plane calculated in CFX.

To conclude, although there are indeed some differences between the CFD simulations and experimental measurements, the overall trend from CFD matches quite well with the test data. CFD modelling can provide very good insights to 


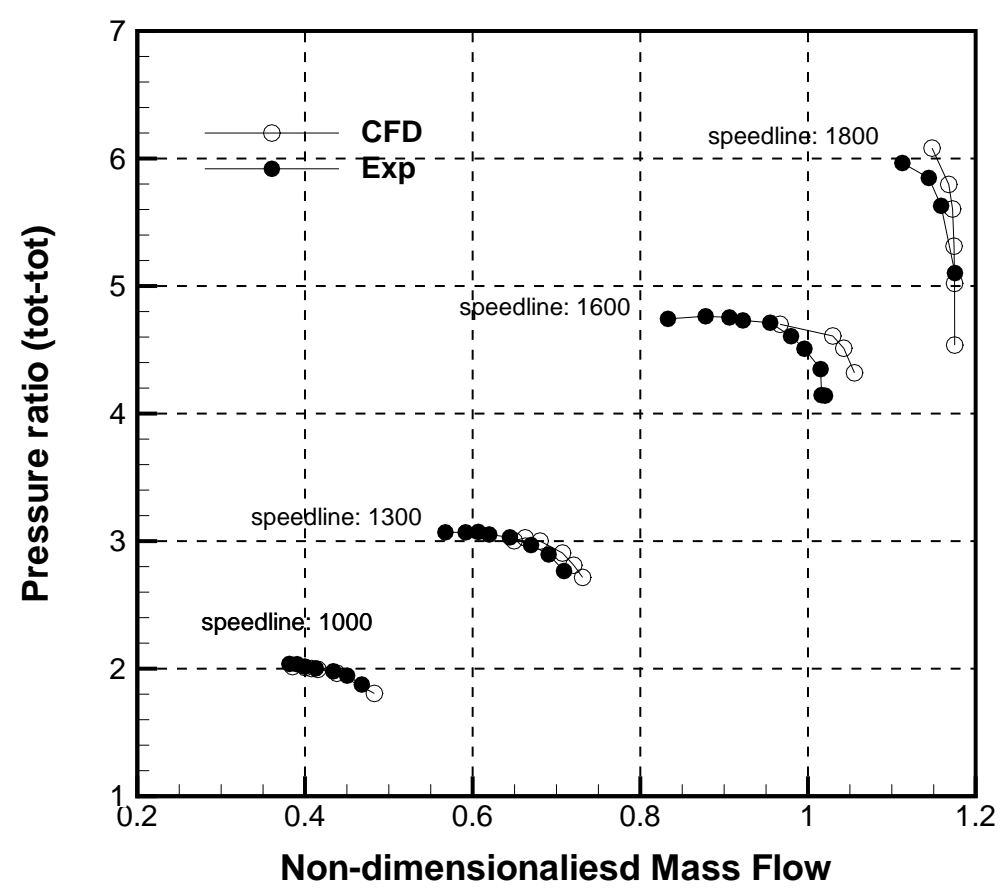

Figure 7.7: Comparisons between the predicted and measured baseline compressor's characteristics: pressure ratio

the compressor performance predictions, and hence the same CFD set-ups will be used for the redesigned compressor stage.

\subsection{Design of the new impeller using TURBOde- $\operatorname{sign} 1$}

In this section the detailed design procedures and input parameters that are needed to solve Eq. 6.87 will be discussed. All the redesigning work was completed using the inverse design code TD1 and its theoretical formulations were explained in great detail in Chapter 6. As has been stated before, the specific design targets are twofold:

- to reduce the aerodynamic noise at top operating speed;

- to enhance the aerodynamic performances at those high operating speeds. 


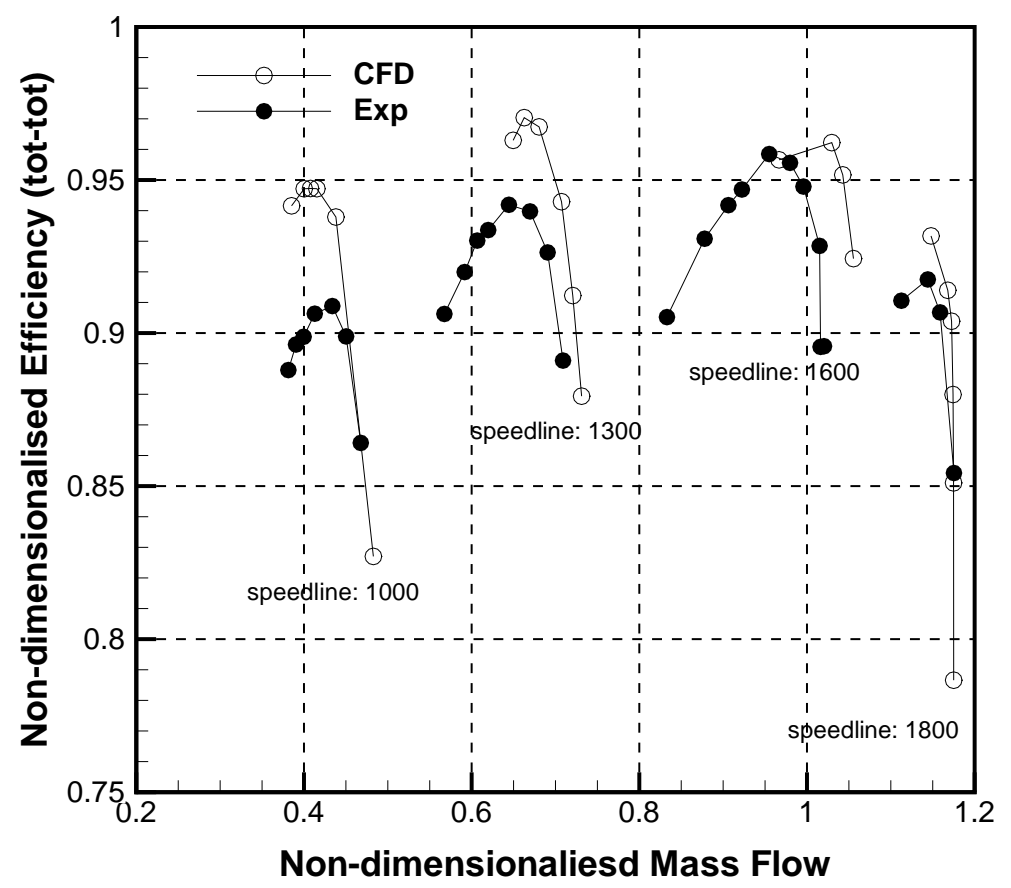

Figure 7.8: Comparisons between the predicted and measured baseline compressor's characteristics: efficiency

\subsubsection{Design point selection}

The choice of the design point on a compressor map has a major impact on the final design's overall performances. The common practice is to let the design point be the peak efficiency point on the most used speedline. This strategy could result in a compressor map with a single point highly optimised but leave the other offdesign points with poor performances. Under this conventional assumption we may choose the BEP on the performance curve of speeline 1800 (that is equivalent to $30 \mathrm{k} \mathrm{rpm}$ ). However designing at this speed would result in a design that is optimised for one point but not at other speedlines. In the work by Zangeneh et al. [2011], it has been confirmed that designing the transonic compressor at $80 \%$ of the full speed can be beneficial for the entire map's overall performances. Thus, we followed the same strategy and decided to design at speedline 1600 (that is equivalent to $26 \mathrm{k} \mathrm{rpm}$, and the maximum allowed speed for the Napier's compressor $32.5 \mathrm{k} \mathrm{rpm}$.) to carry out the current design. Figure 7.9 shows the predicted pressure ratio and efficiency characteristics against mass flow rate at 


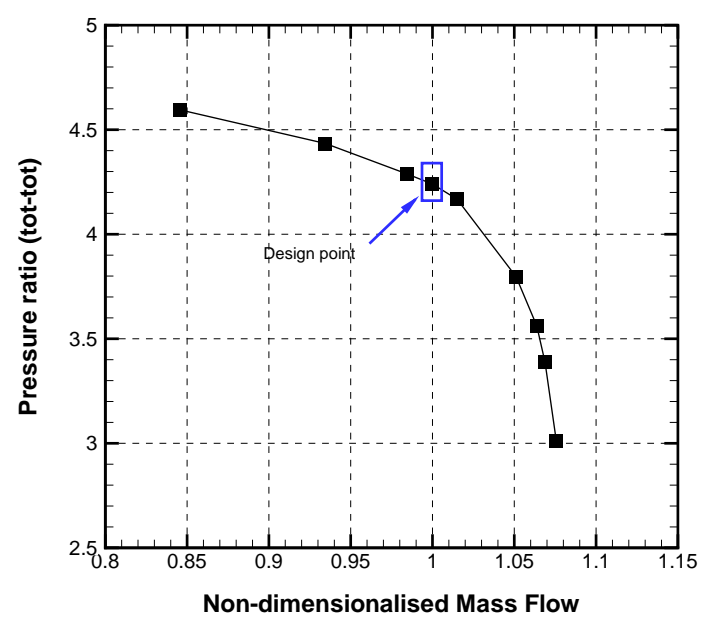

(a) Predicted pressure ratio measured at impeller exit

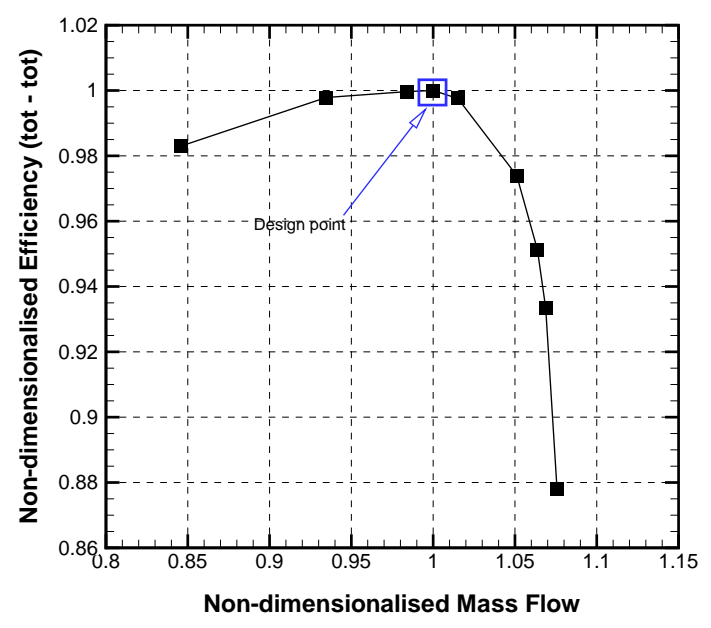

(b) Predicted efficiency measured at impeller exit

Figure 7.9: CFD prediction of Napier baseline impeller performances at $26 \mathrm{krpm}$

this speed. The BEP, corresponding to the highlighted point, was selected as the final design point.

\subsubsection{Meridional contour and number of blades}

The hub and shroud sections for the new impeller wheel were kept the same as the baseline, as can be seen Fig. 7.10. The number of blades is also kept the same as the baseline wheel, i.e., 11 full/splitter blades. It should be mentioned that it is also possible to perform the optimisation including the simultaneous variation of both meridional housing and blade loading parameters, which is a more complicated hybrid optimisation scheme. This strategy is fully achievable in another module of TURBddesign Suite, namely, TURBOdesign Optima. However, in the current study no effort was made to include the change of the meridional housing for the new impeller design.

\subsubsection{Thickness distribution}

The thickness of the full and splitter blades were specified individually as inputs for the TD1 code. The thickness distribution was first extracted from the baseline impeller and it was decided to increase the hub thickness by $15 \%$ to avoid the 


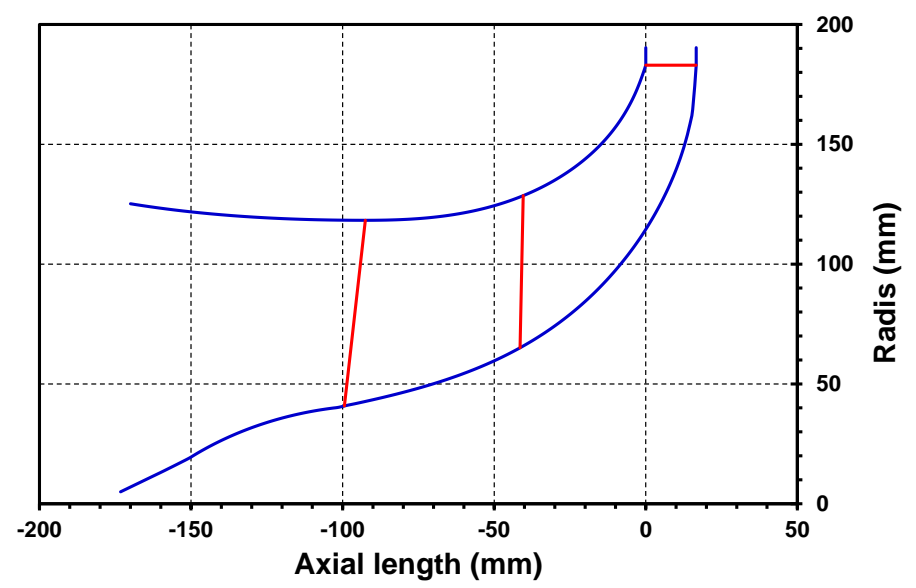

Figure 7.10: Meridional contour for the inverse designed impeller

high stress issues during the design process, as can se been in Figs. 7.11 and 7.12, in which the normal thickness at different spanwise position are plot against the normalised meridional length.

\subsubsection{Inlet boundary conditions}

The boundary conditions specified at the inlet include the total temperature, total pressure and volume flow rate. The inlet total temperature and pressure are set to be $298 \mathrm{~K}$ and $101.135 \mathrm{kPa}$. The volume flow rate is calculated using the mass flow of the design point highlighted in Fig. 7.9 and air density.

The inlet velocity profile is set to be perfectly uniform without any swirl velocity, thus the distribution of $r V_{\theta}^{*}$ from hub to shroud at the impeller leading edge is zero throughout.

\subsubsection{Blockage factor}

In the presence of transonic flows inside blade cascades, a displacement thickness of boundary layer is always observed. This thickness works as a blockage to reduce the effective flow area, for which Khalid et al. [1999]; Suder [1997] conducted thorough studies and quantified it as

$$
B L K=1-\frac{\text { effective area }}{\text { geometric area }}=1-\frac{A_{g}-\int \delta^{*} d r}{A_{g}}
$$



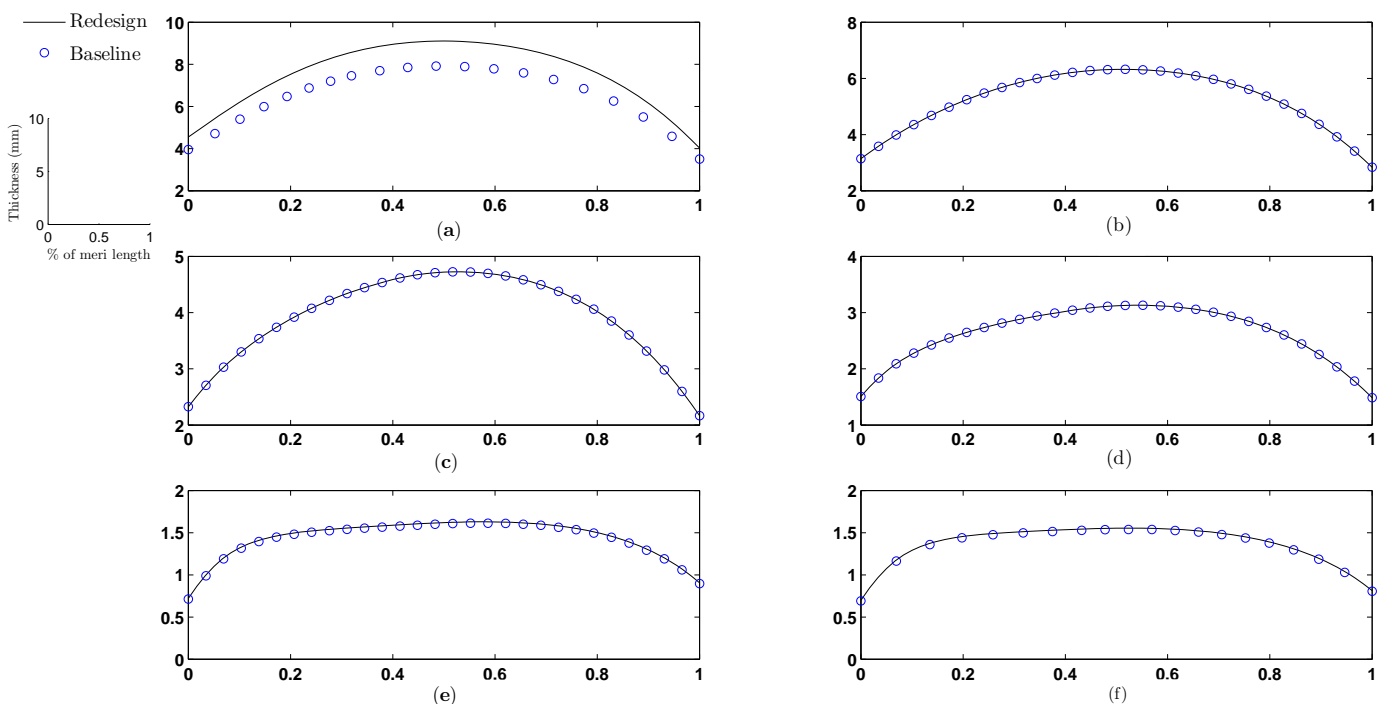

Figure 7.11: Comparisons of full blade thickness at different spanwise height: (a) Hub section; (b)25\% spanwise height; (c) $50 \%$ spanwise height; (d)75\% spanwise height; (e) $98 \%$ spanwise height; (f)Shroud section
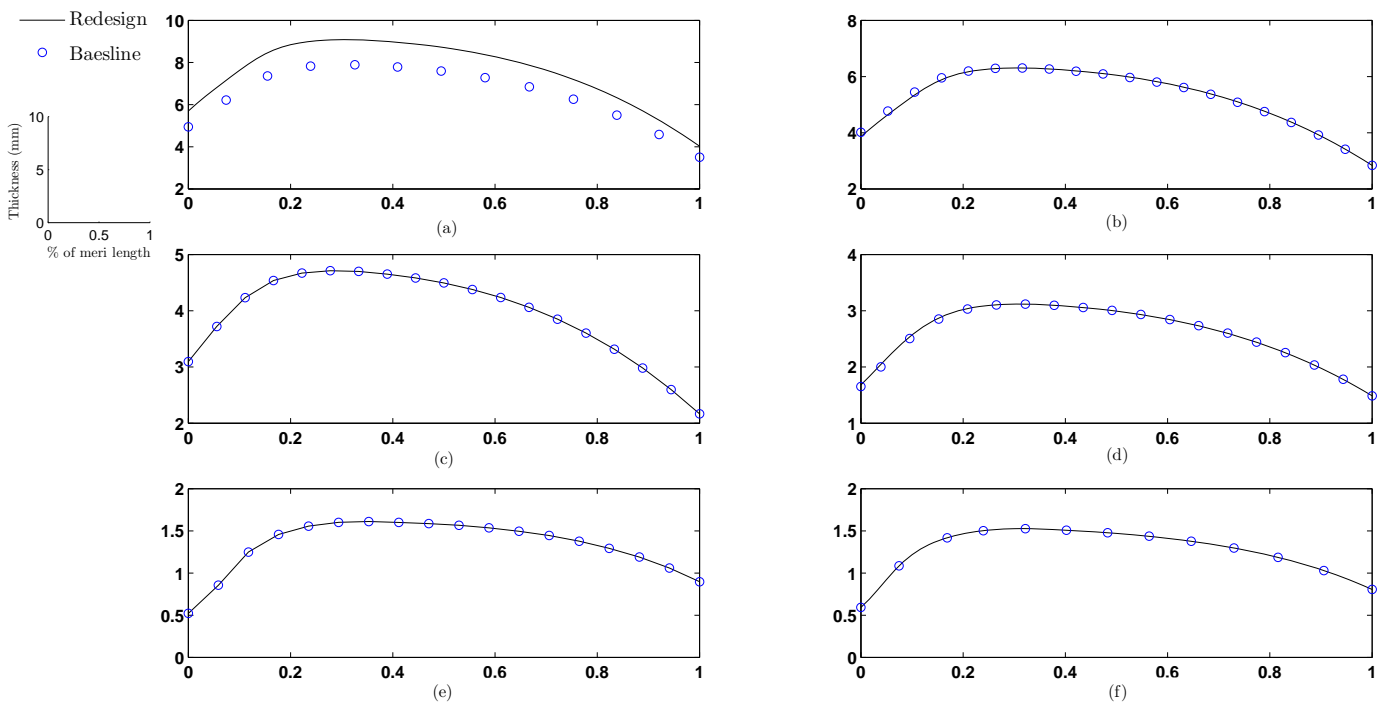

Figure 7.12: Comparisons of splitter blade thickness at different spanwise height: (a) Hub section; (b)25\% spanwise height; (c)50\% spanwise height; (d)75\% spanwise height; (e)98\% spanwise height; (f)Shroud section 


\section{DESIGN AND ANALYSIS OF A NEW TRANSONIC CENTRIFUGAL IMPELLER}

where $B L K$ represents the blockage, $A_{g}$ is the total geometric area and $\delta^{*}$ is the integral of the velocity-density deficit across the rotor passage, for which Suder defined it as

$$
\delta^{*}(r)=\int_{0}^{2 \pi / B}\left(1-\frac{\rho u}{(\rho u)_{\text {inviscid }}}\right) r d \theta .
$$

From a design perspective, it is necessary to involve such an effect. But TD1's solver does not include any viscosity, thus, no boundary layer displacement thickness will be modelled. In order to take account of this important phenomenon, a blockage factor must be introduced. However, in case of centrifugal compressors, the flow non-uniformity occupies the entire blade channel, consequently causing difficulty in defining the blockage. An alternative way suggested by Dring [1984] is used in TD1 code, which is

$$
B L K=\frac{\overline{V_{\mathrm{m}}^{\text {area }}}}{\overline{V_{\mathrm{m}}^{\text {mass }}}},
$$

where

$$
\begin{aligned}
\overline{V_{\mathrm{m}}^{\text {area }}} & =\frac{\int_{0}^{2 \pi / B} V_{m} r d \theta}{\int_{0}^{2 \pi / B} r \theta}, \\
\overline{V_{\mathrm{m}}^{\text {mass }}} & =\frac{\int_{0}^{2 \pi / B} \rho V_{m}^{2} r d \theta}{\int_{0}^{2 \pi / B} \rho V_{m} r d \theta},
\end{aligned}
$$

where $\overline{V_{\mathrm{m}}^{\text {area }}}$ and $\overline{V_{\mathrm{m}}^{\text {mass }}}$ represent the area and mass average of meridional velocity respectively. Figure 7.13 shows the blockage factor distribution from the inlet to outlet used in the current design. 


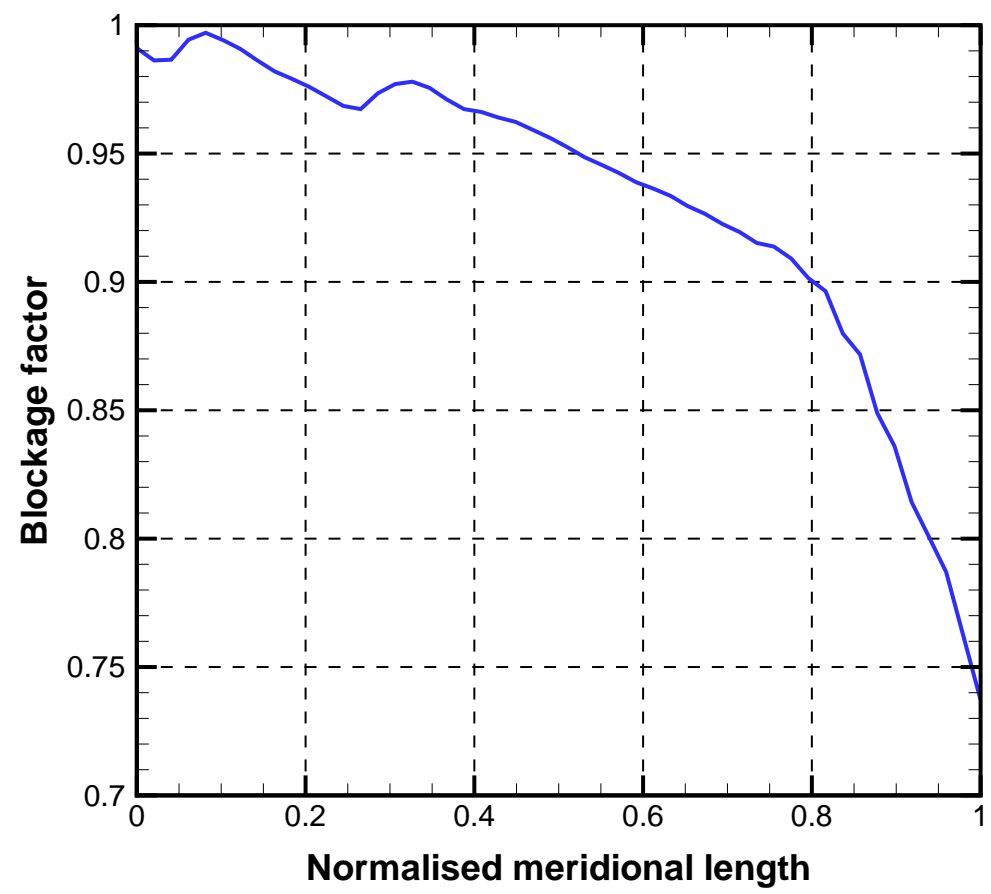

Figure 7.13: Blockage factor distribution from the inlet to outlet

\subsubsection{Blade loading}

\subsubsection{Spanwise $r V_{\theta}^{*}$}

As has been explained in the last chapter, TD1 allows a designer to design the full and splitter blade independently according to the specified spanwise swirl velocity $r V_{\theta}^{*}$ and streamwise distribution of $\partial\left(r V_{\theta}^{*}\right) / \partial m^{*}$. A parameter $\kappa$ has been introduced to control the allocation of bound circulation on the full and splitter blade surface, which is defined as

$$
\kappa=\frac{\left(r V_{\theta}^{*}\right)^{\text {splitter }}}{\left(r V_{\theta}^{*}\right)^{\text {splitter }}+\left(r V_{\theta}^{*}\right)^{\text {full }}} .
$$

The splitter work ratio can be independently specified at the hub and shroud section and initially it can be based on the splitter's meridional length $\lambda$, which is defined, in a similar manner to $\kappa$, as the ratio between the splitter meridional length and the sum of full and splitter meridional length. Once the meridional contour is imported to TD1, as is shown in Fig. 7.10, the length ratio $\lambda$ will 


\section{DESIGN AND ANALYSIS OF A NEW TRANSONIC CENTRIFUGAL IMPELLER}

Table 7.3: Spanwise $r V_{\theta}^{*}$ distribution

\begin{tabular}{|c|c|c|c|}
\hline & Hub & Shroud & Average \\
\hline$r V_{\theta}^{*}$ at LE & 0 & 0 & 0 \\
\hline$r V_{\theta}^{*}$ at TE & 0.572 & 0.52 & 0.546 \\
\hline Splitter work ratio $(\kappa)$ & 0.3190 & 0.3185 & 0.3187 \\
\hline Length ratio $(\lambda)$ & 0.4133 & 0.3730 & 0.3985 \\
\hline
\end{tabular}

be automatically calculated. In Table $7.3 r V_{\theta}^{*}$ and splitter work ratios at hub and shroud are summarised. In this case, the average splitter work ratio $\kappa$ was initially set to 0.4 to match the meridional length ratio $\lambda$ but was fine-tuned to about 0.32 for better performances. It will be explained with the flow field details around the splitter blade that the splitter work ratio can modulate the flow behaviours effectively so as to improve stage performances.

Regarding the total $r V_{\theta}^{*}$ at the impeller TE, a non-free vortex distribution was adopted. One can see in Table 7.3 that a larger $r V_{\theta}^{*}$ was specified at the hub. This arrangement is useful to reduce the loading on the shroud section that would result in weakened shock strength on the inducer and reduction of tip leakage flows. The final values of $r V_{\theta}^{*}$ at the impeller TE depend on the efficiency and pressure ratio of the impeller and were fine-tuned for the optimal stage performances.

\subsubsection{Streamwise $\partial\left(r V_{\theta}^{*}\right) / \partial m^{*}$}

The other important design parameter is the streamwise blade loading, or more precisely, $\partial\left(r V_{\theta}^{*}\right) / \partial m^{*}$ from the LE to TE. The choice of the blade loading distribution has a dominant effect on determining the compressor performances. Depending on the speed and size of the impeller, the blade loading used can be quite different. For example, Zangeneh et al. [2010] presented a very effective distribution for a 4.5 pressure ratio turbocharger compressor, which is shown in Fig. 7.14. This distribution assumes a strong aft-loading and weak leading edge loading to mitigate the strong shock losses occurring at the inducer. For a transonic turbocharger compressor, the shock losses at the inducer are usually dominant. The impeller designed was manufactured and tested by Cummins Turbo Technology. A considerable gain of efficiency was obtained throughout most area of 


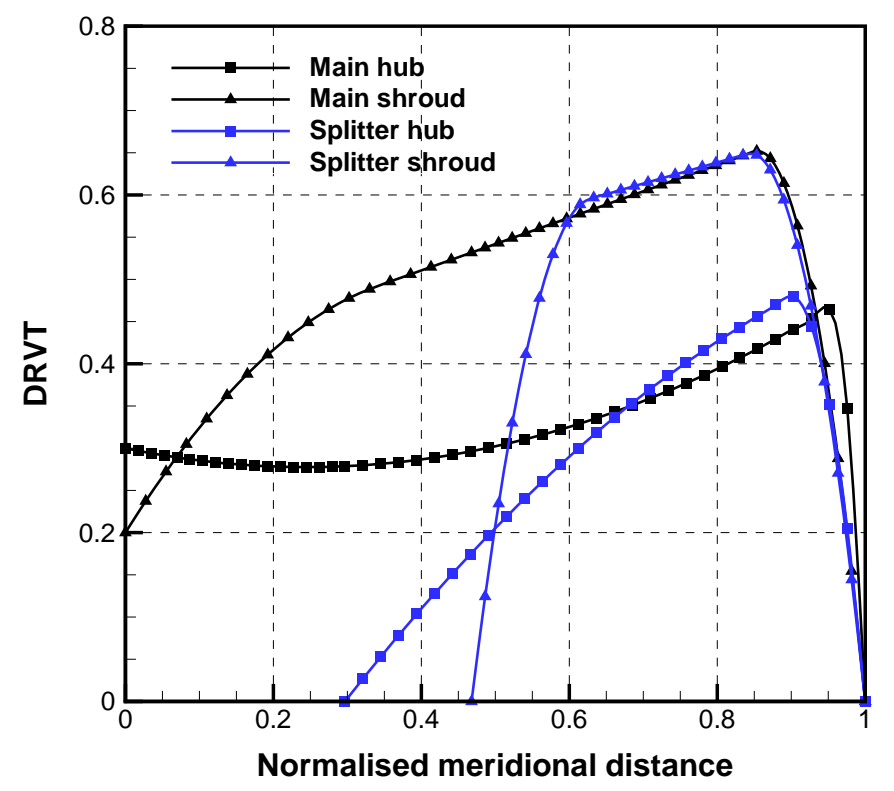

Figure 7.14: Blade loading for a 4.5 pressure ratio centrifugal impeller

the compressor map. However, by using this loading specification the compressor range was similar to the baseline design.

Later on, in the work by Zangeneh et al. [2011] a different type of blade loading was used to redesign the well-known SRV2 impeller that was initially designed by DLR. This redesign was very successful in terms of both pressure ratio and efficiency, which were increased significantly at all three operating speeds. The blade loading used for the SRV2 impeller redesign shown in Fig. 7.15 assumed a strong incidence $\left(\partial\left(r V_{\theta}^{*}\right) / \partial m^{*}\right.$ at $\left.m^{*}=0\right)$ at the full blade LE on the hub and midspan section to get enough throat area so as to maintain the choke margin. Following that, a very rapid unloading on the hub and midspan is enabled to avoid the strong shock waves. In order to minimise the secondary flows on the suction surface, it is recommended by Zangeneh et al. [1998] to use an aft-loading on the hub and fore-loading on the shroud. But in this transonic case, strong fore-loading at the shroud can result in a very strong shock. Thus, a strong aftloading is used at the hub with a moderately aft-loaded shroud. The splitter blade loading distribution adapted more or less the same profile as the full blade in order to have a similar blade geometry to the full blade. If too distinct distributions are used, the full and splitter blade geometries will be very different and costly 


\section{DESIGN AND ANALYSIS OF A NEW TRANSONIC CENTRIFUGAL IMPELLER}

for manufacturing. In a subsequent paper by Wang \& Zangeneh [2014] flow field details were compared to understand why the redesigned blade was able to improve the performances, which were also presented in Chapter 5. Additionally, the aeroaocustic calculations were also performed in their work. The acoustic calculations confirmed that the redesigned impeller can also lead to a lower noise level. Provided that the SRV2 compressor has similar tip speed and pressure ratio to the current compressor, the blade loading used in Fig. 7.15 is therefore a good starting point to the current redesign work. However, one must notice that the redesigned SRV2 impeller has a smaller surge margin (Wang \& Zangeneh [2014]; Zangeneh et al. [2011]) comparing with the baseline while the choke margin was bigger, which is likely due to the overloading on the leading edge and inducer, as one can see in Fig. 7.15. On one hand, the throat is opened up to pass more flows. On the other hand, the overloaded LE results in a smaller blade angle and hence an overall bigger attack angle which leads to the earlier stalling. Conclusively, the prescription of blade loading in current design work needs to be modified accordingly in order to avoid the similar earlier-stalling problem.

In the current work the two major targets are to reduce the compressor noise and improve the compressor efficiency at higher speeds. Thus, an aft-loaded distribution should be imposed to avoid the strong shocks on the inducer as they are the important sources responsible for strong noise and efficiency losses. Regarding the LE loading, as it directly affects the throat area and hence the choke margin, the final leading edge loading can be gradually obtained by looking at the calculated choke margins and noise levels. The loading on the splitter blade can be prescribed in a similar way. The detailed scheme of the blade loading iterations are given below:

- DRVT at LE $\left(\partial\left(r V_{\theta}^{*}\right) / \partial m^{*}\right.$ at $\left.m^{*}=0\right)$. Since the TD1 code provides information on the throat area for every design, DRVT at hub, midspan and shroud can be micro-adjusted to ensure that the throat area is fairly close to that of the baseline design. It is effective to use a similar combination of hub to shroud DRVT at LE to the SRV2 blade loading in Fig. 7.15. However, as we explained previously, the redesigned SRV2 impeller's surge margin was not as good as the baseline one. Thus, a weak DRVT was deliberately 


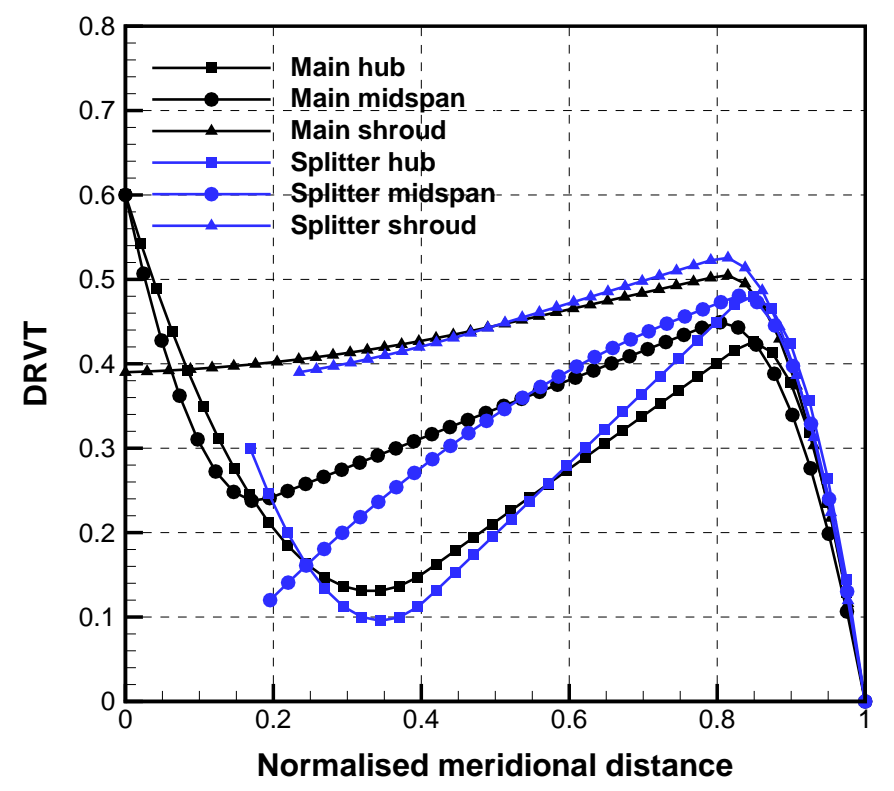

Figure 7.15: Blade loading for the redesigned SRV2 centrifugal impeller

imposed on the shroud section, which was to reduce the incidence angle near the shroud section where the compressor stalling often occurs.

- NC. This parameter determines the loading on the inducer. Figure 7.15 presents a strong DRVT and small NC to open the throat first and then rapidly unload the inducer. This set-up has effectively achieved the wanted throat area and weakened shock strength for the redesigned SRV2 impeller. But in the current design, a moderately bigger NC may be required since the DRVT on the hub and midspan section was not as strong as the SRV2 case. Particularly, a very weak DRVT at the shroud was intended to be used, which would close the channel and consequently reduce the throat area. Thus, NC can be fine-tuned to ensure the inducer is opened up enough to achieve the desired throat area with a weak DRVT imposed on the shroud section.

- Slope. The slope of the blade loading curve is quite essential as it defines whether the loading distribution is aft- or fore-loaded. As has been explained, a wider operating range and reduced shock strength would require the slope to be positive, i.e., aft-loading. One should bear in mind that an 


\section{DESIGN AND ANALYSIS OF A NEW TRANSONIC CENTRIFUGAL IMPELLER}

over aft-loaded distribution may also cause strong profile losses and give rise to a big turn on the blade profile near the trailing edge, which results in flow separations and secondary flows. (Zangeneh et al. [1998]) The Slope magnitude can be used to modulate the blade loading between the inducer and exducer.

- ND. Combining with Slope, this parameter can define the strength of loading on the exducer. Suppose a very strong aft-loading on the exducer is produced, i.e., big slope and ND, the blade profile near the trailing edge will have a very rapid turn, which will cause flow separations towards to the trailing edge. In addition to that, there will be very strong tip leakage flow interactions near the blade TE, which will harm the exit flow uniformity and increase the mixing losses in the diffuser, consequently causing poor stage performances.

Figure 7.16 shows the final blade loading used for the redesign. As one can see strong LE loading is applied to both hub and midspan section of full blade, while a very weak DRVT at shroud LE is imposed. This arrangement of LE DRVT is to get the needed throat area and reduce the shocks strength at LE as much as possible. A moderately aft-loading distribution is applied to both hub and shroud while a very strong aft-loading is specified on the shroud section. The loading on the splitter blade adopts the same pattern except that the a strong incidence is applied on its shroud section, which is to open up the throat of the channel formed between the full and splitter.

\subsubsection{Stacking}

The final important input parameter is the impeller stacking angle $\theta_{s k}$ at the trailing edge, which is shown in Fig. 7.17. Its distribution from hub to shroud is used as initial conditions for the hyperbolic partial differential equation used to compute the blade wrap angles at other streamwise locations. In this case the full and splitter blades were stacked in such a way that the shroud leads the hub in the direction of rotation by 5 degrees. This type of stacking condition is similar to the conventional rake and is used primarily to reduce the stresses at 


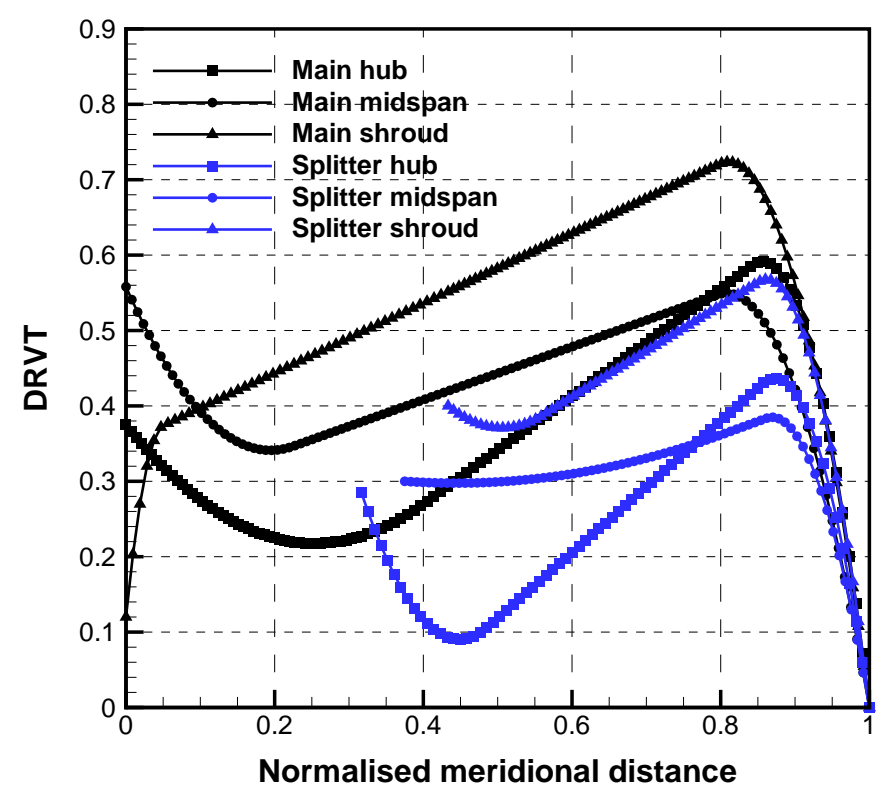

Figure 7.16: Optimal blade loading for the final redesign

the impeller tip region. Further more it can help to reduce the leakage flow. This "shroud leads the hub" rake angle at TE is used widely in conventional designs and Napier's baseline impeller also adopted this approach.

\subsection{Study of impeller structure integrity}

As the design flow chart suggests once a design was created by TD1, its mechanical feasibility will be first checked. This section presents the FEA analysis for both impeller wheels. First, a simplified model was used to study the structure integrity of the redesigned impeller and confirm if its mechanical strengths meet Napier's requirement. The simplified model uses only one pair of full and splitter blades with a fixed support set at the blade root. Once it is confirmed by the simple FEA that the redesigned impeller is mechanically acceptable, a much more rigorous FEA appraisal was conducted by Napier Turbochargers, including the calculations for the back plate, thermal expansion effect, and the fillet on the blade root, to confirm if the impeller wheel can meet Napier's internal criterion of manufacturability. Figure 7.18 shows the set up for the simplified FEA model, in which the red parts at the full and splitter blade roots represent the fixed 


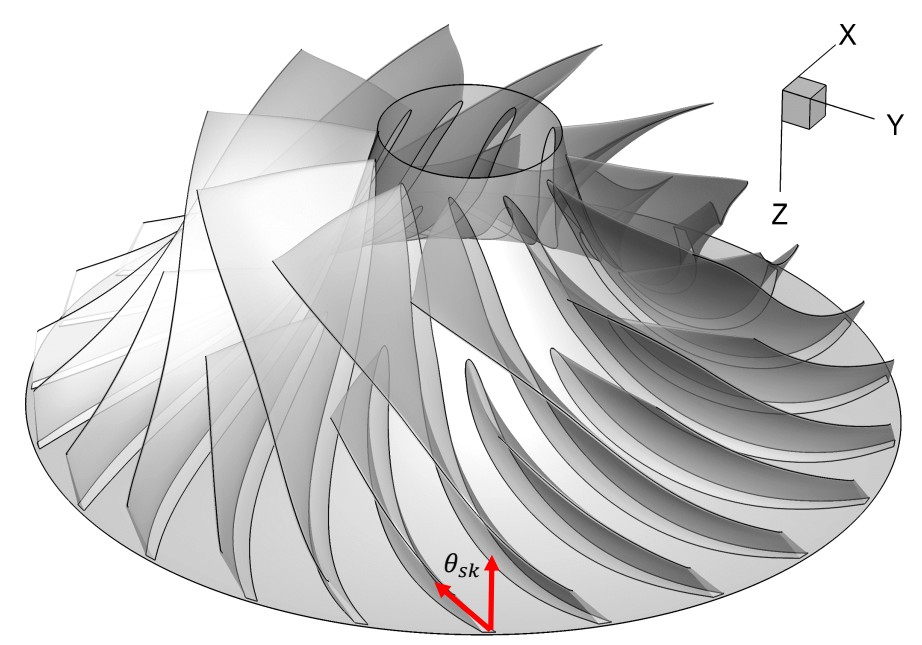

Figure 7.17: Illustration of the definition of stacking angle in TD1

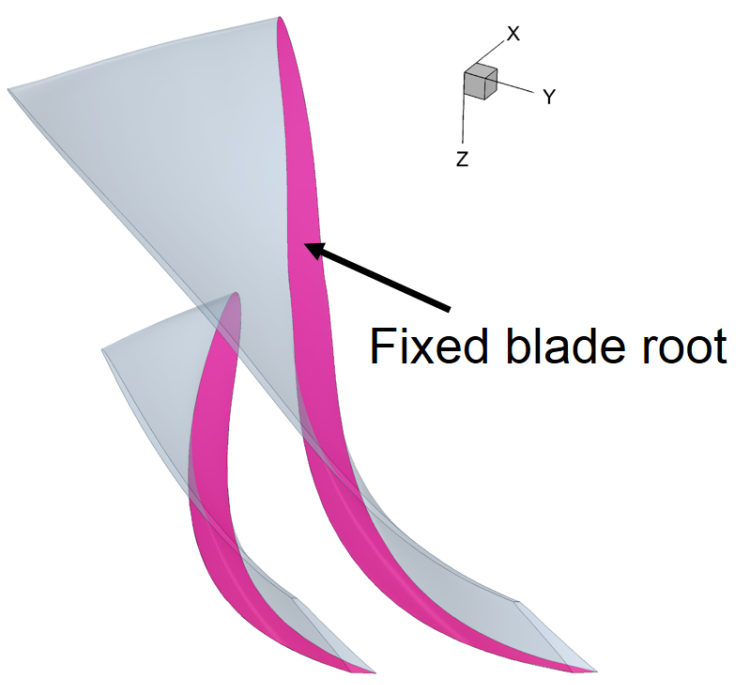

Figure 7.18: Simplified FEA set up. $\omega=32.5 \mathrm{krpm}$ 


\section{DESIGN AND ANALYSIS OF A NEW TRANSONIC CENTRIFUGAL IMPELLER}

Table 7.4: Aluminium alloy 2618A mechanical properties: no thermal effects considered

\begin{tabular}{ll} 
Density & $2770 \mathrm{~kg} / \mathrm{m}^{3}$ \\
Young's/Elastic modulus & $69.9 \mathrm{GPa}$ \\
Yield strengh & $369 \mathrm{MPa}$ \\
Poisson's ratio & 0.33 \\
\hline
\end{tabular}

support. The blades are set to rotate with the maximum compressor speed 32.5 krpm around z-axis. The Aluminium alloy 2618A was used for making both baseline and redesign wheels whose mechanical properties are give in Table 7.4 below. There are three important factors that determine the mechanical acceptability, which are

1. von Mises stress limit: it must be below a level to give adequately low cycle fatigue life;

2. $1^{\text {st }}$ natural frequency needs to be greater than 4 times of the rotating frequency, i.e. $2167 \mathrm{~Hz}$ in the current case;

3. the blade deflection needs to be as small as possible. If the blade deflection is too large the CFD calculations will deviate from the reality too much since CFD simulations assumes the absolute rigidity of the blades. The deflected blade will distort the flow field considerably, resulting in very different compressor characteristics from expectations.

The ANSYS Mechanical Pre-stress Modal was used for the FEA calculations. Table 7.5 summarises the results from the simplified FEA modelling. As can be seen, the redesigned impeller has advantages for all three factors. In Figs. 7.19a, $7.19 \mathrm{~b}, 7.20 \mathrm{a}$ and $7.20 \mathrm{~b}$ are the comparisons of the normalised von Mises stress and total deformations, which have been divided by the maximum stress and deformation. Both baseline and redesigned impellers deflect most in the vicinity of the full blade tip, and it is clear that the baseline one has noticeable bigger deformations comparing with the redesigned one.

A more rigorous FEA evaluation was then subsequently conducted by Napier Turbochargers for the proposed impeller designed by the inverse design method. 

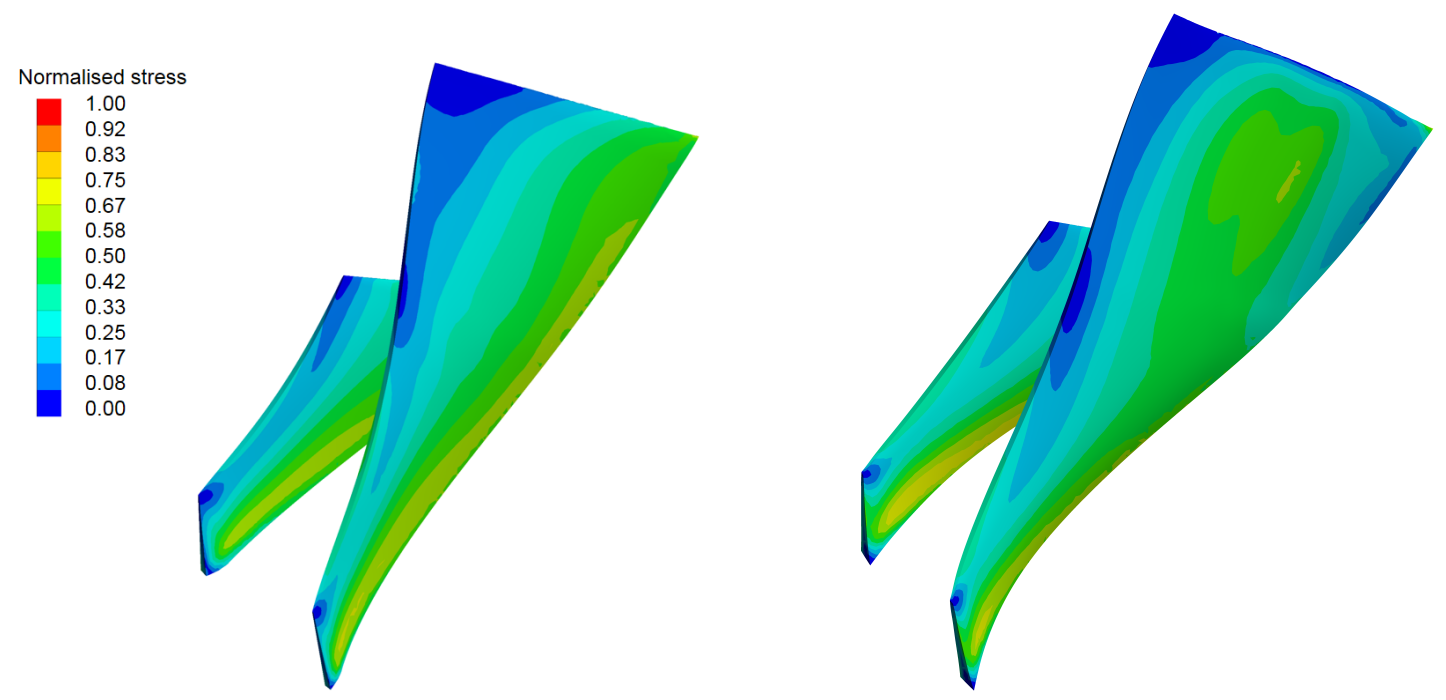

(a) von Mises stress contour on the baseline (b) von Mises stress contour on the reimpeller surface designed impeller surface

Figure 7.19: Comparisons of the von Mises stress contour on the blade surface
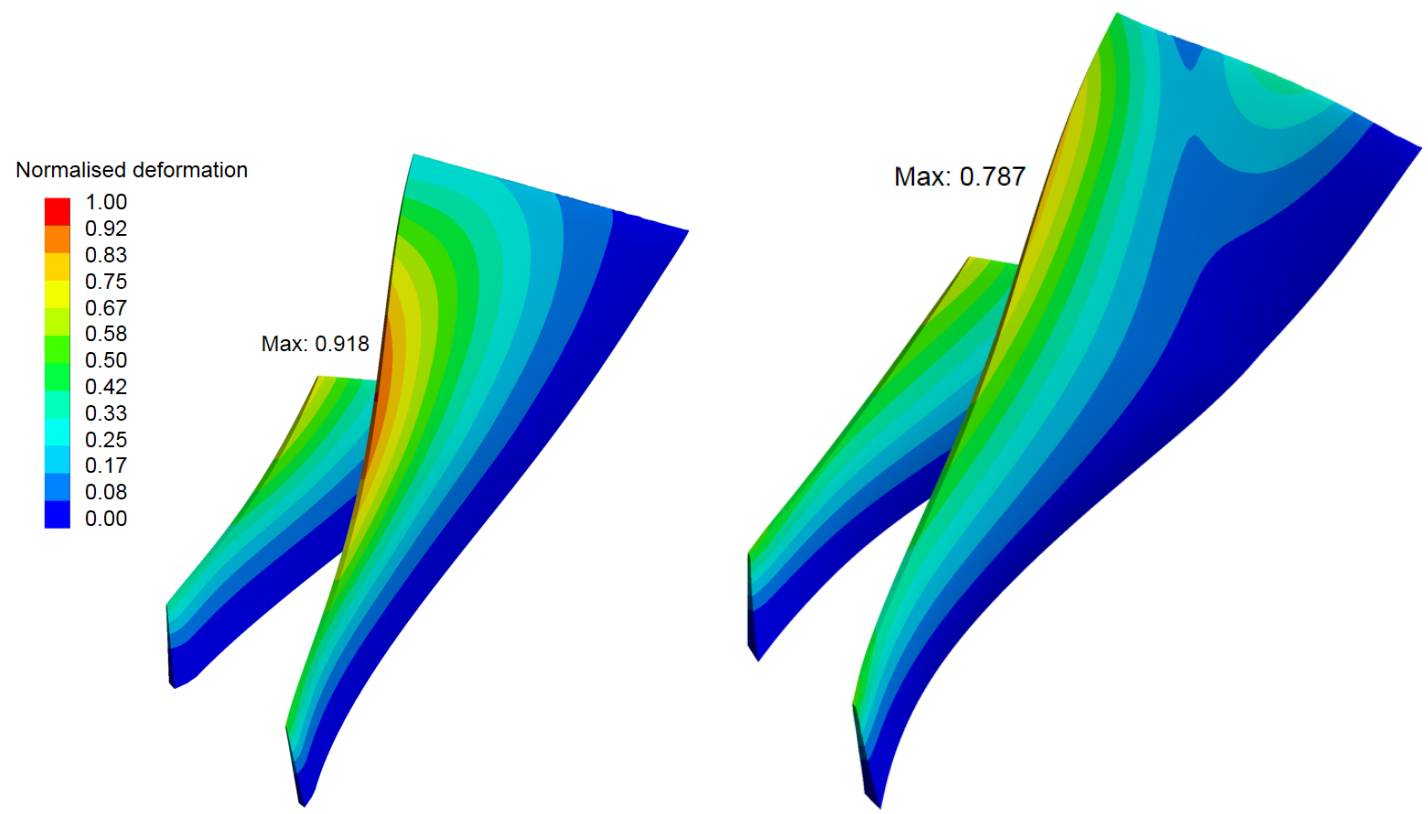

(a) Deformation on the baseline impeller (b) Deformation on the redesigned impeller surface surface

Figure 7.20: Comparisons of the deformation contour on the blade surface 


\section{DESIGN AND ANALYSIS OF A NEW TRANSONIC CENTRIFUGAL IMPELLER}

Table 7.5: Normalised results from the simplified FEA modelling

\begin{tabular}{llll}
\hline & von-mises stress & Max deflection & $1^{s t} f_{n}$ \\
\hline Baseline & 0.94 & 0.92 & 0.88 \\
Redesign & 0.88 & 0.78 & 0.95 \\
\% of change & $-6.82 \%$ & $-17.95 \%$ & $+7.37 \%$ \\
\hline
\end{tabular}

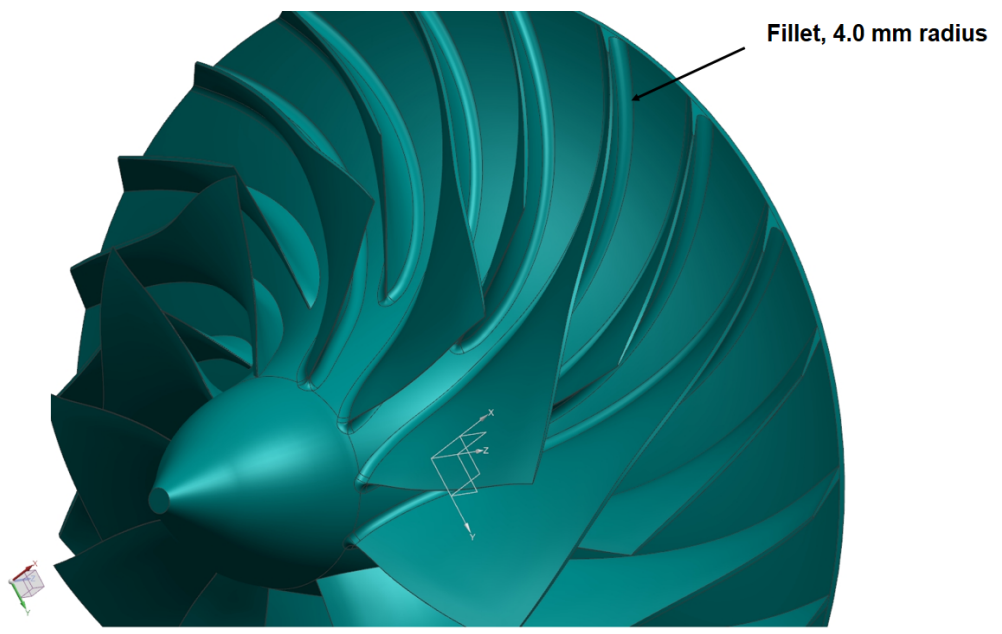

Figure 7.21: Full impeller wheel with fillets used for FEA analysis

The thermal effects on the material properties were taken account of. In Table 7.6 are shown the thermodynamic properties of the Aluminium Alloy 2618A at constant temperature. In Napier's FEA calculations, the variations of isotropic elasticity and bilinear kinematic hardening against temperatures were also included. The details of the model and temperature dependence of the mechanical strength are commercially sensitive, so the absolute values are not reproduced here. Figure 7.21 shows the full impeller wheel used for the FEA by Napier, and it has a fillet with radius $4.0 \mathrm{~mm}$. The mesh elements used on the blades have a maximum size $2 \mathrm{~mm}$ while on the fillets the maximum mesh size is $1 \mathrm{~mm}$. The total element and nodal counts for this model are 388661 and 569093 respectively, which can be seen in Fig. 7.22. Figure 7.23 presents the thermal analysis of the impeller wheel and the temperature distribution across the whole model is demonstrated. 


\section{DESIGN AND ANALYSIS OF A NEW TRANSONIC CENTRIFUGAL IMPELLER}

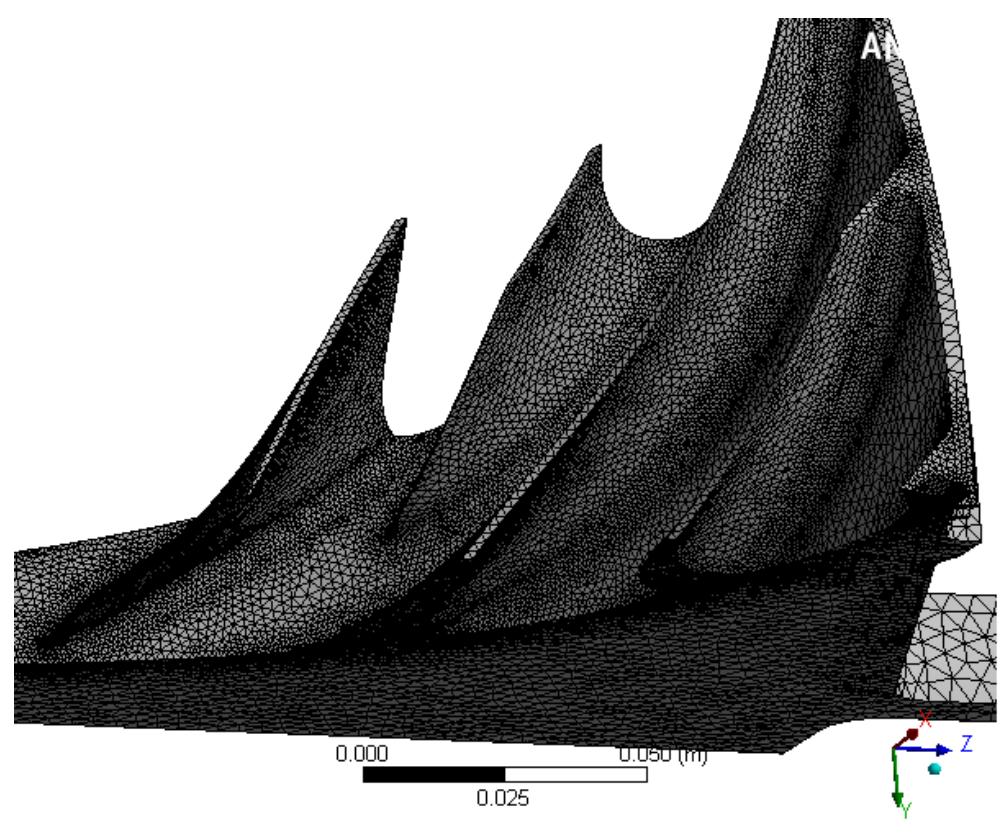

Figure 7.22: Full impeller wheel mesh used for FEA analysis

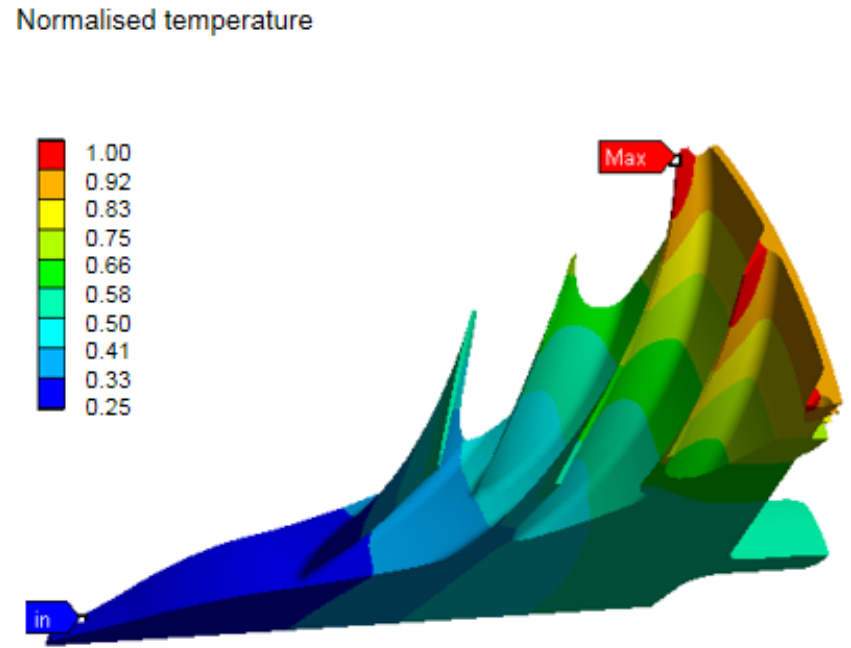

Figure 7.23: Thermal analysis for the redesigned full impeller wheel 


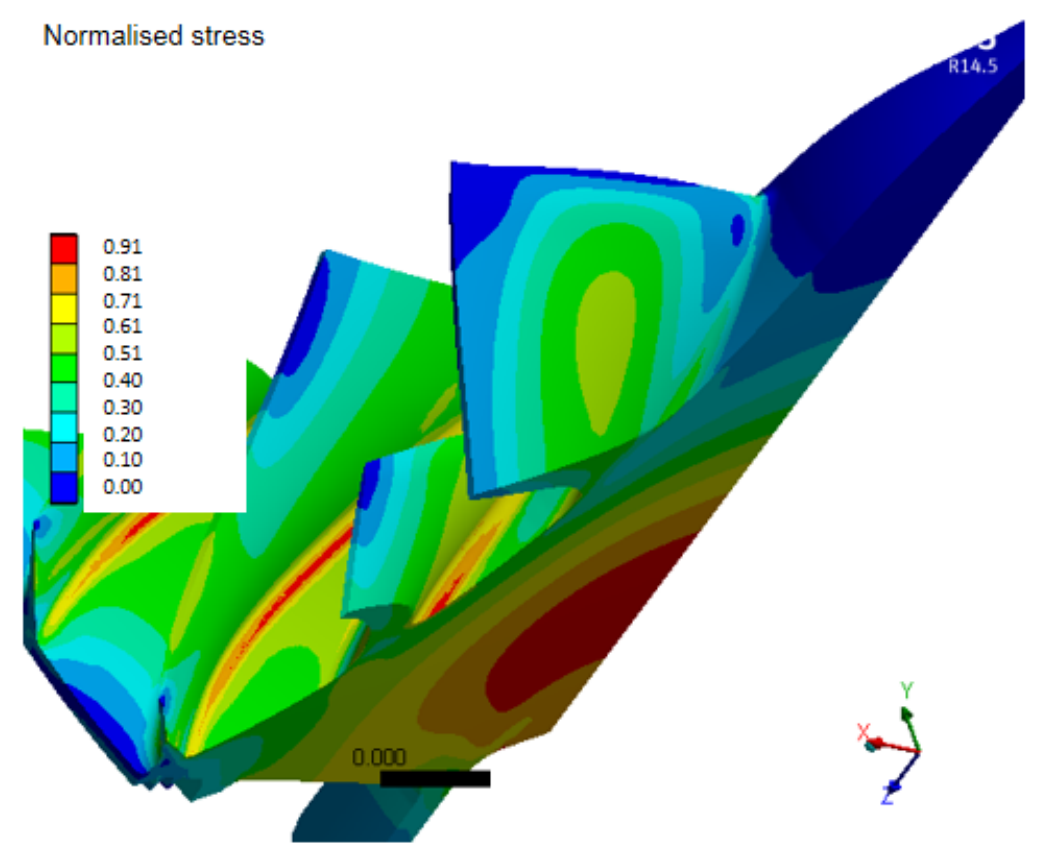

Figure 7.24: Stress analysis for the redesigned full impeller wheel

Table 7.6: Material constants of Aluminium alloy 2618A

\begin{tabular}{|l|l|}
\hline \multicolumn{2}{|c|}{ Materials constant } \\
\hline Density & $2770 \mathrm{~kg} / \mathrm{m}$ \\
\hline Specific Heat & $875 \mathrm{~J} \mathrm{~kg}^{-1} \mathrm{C}^{-1}$ \\
\hline Thermal Conductivity & $190 \mathrm{~W} \mathrm{~m}{ }^{-1} \mathrm{C}^{-1}$ \\
\hline Coefficient of Thermal Expansion & $2.27 \times 10^{-5} \mathrm{C}^{-1}$ \\
\hline
\end{tabular}

Figure 7.24 shows the normalised stress distributions on the wheel and the peak stress is 0.91 on the fillet of the suction surface of the slitter blade, which is slightly higher than the prediction of the simplified FEA modelling. Figure 7.25 shows deflection distribution of the blades. A normalised deflection of 0.493 was found at the inlet whereas it was 0.583 for the baseline impeller.

Figure 7.26 confirms that the $1^{\text {st }}$ natural frequency at all rpms is greater than $2267 \mathrm{~Hz}$, which is four times of the highest rotation frequency, thus, there is enough margin before any excitation.

After discussing the design process in detail, particularly on the blade loading prescription, and confirming the redesigned blade is mechanically acceptable , 


\section{DESIGN AND ANALYSIS OF A NEW TRANSONIC CENTRIFUGAL IMPELLER}

Normalised deformation

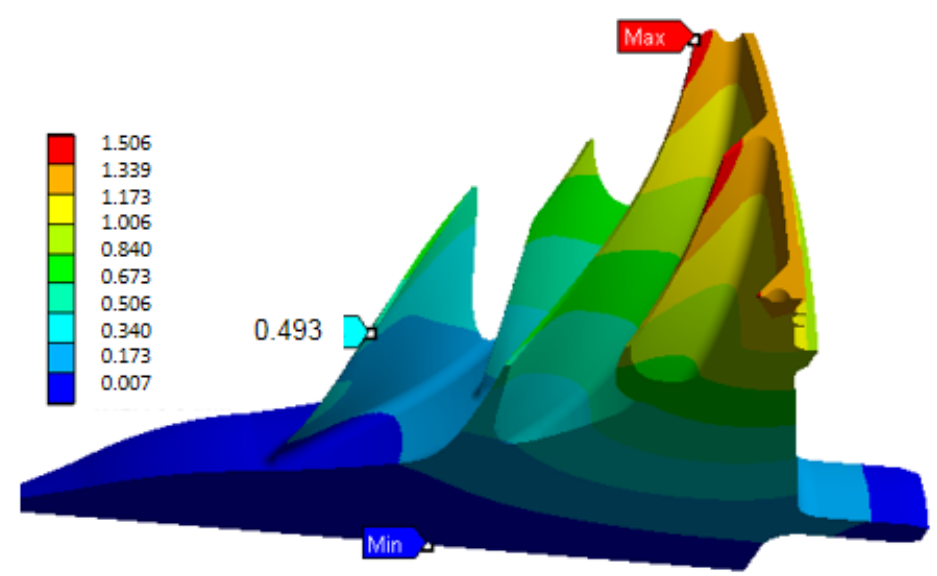

Figure 7.25: Deformation analysis for the redesigned full impeller wheel

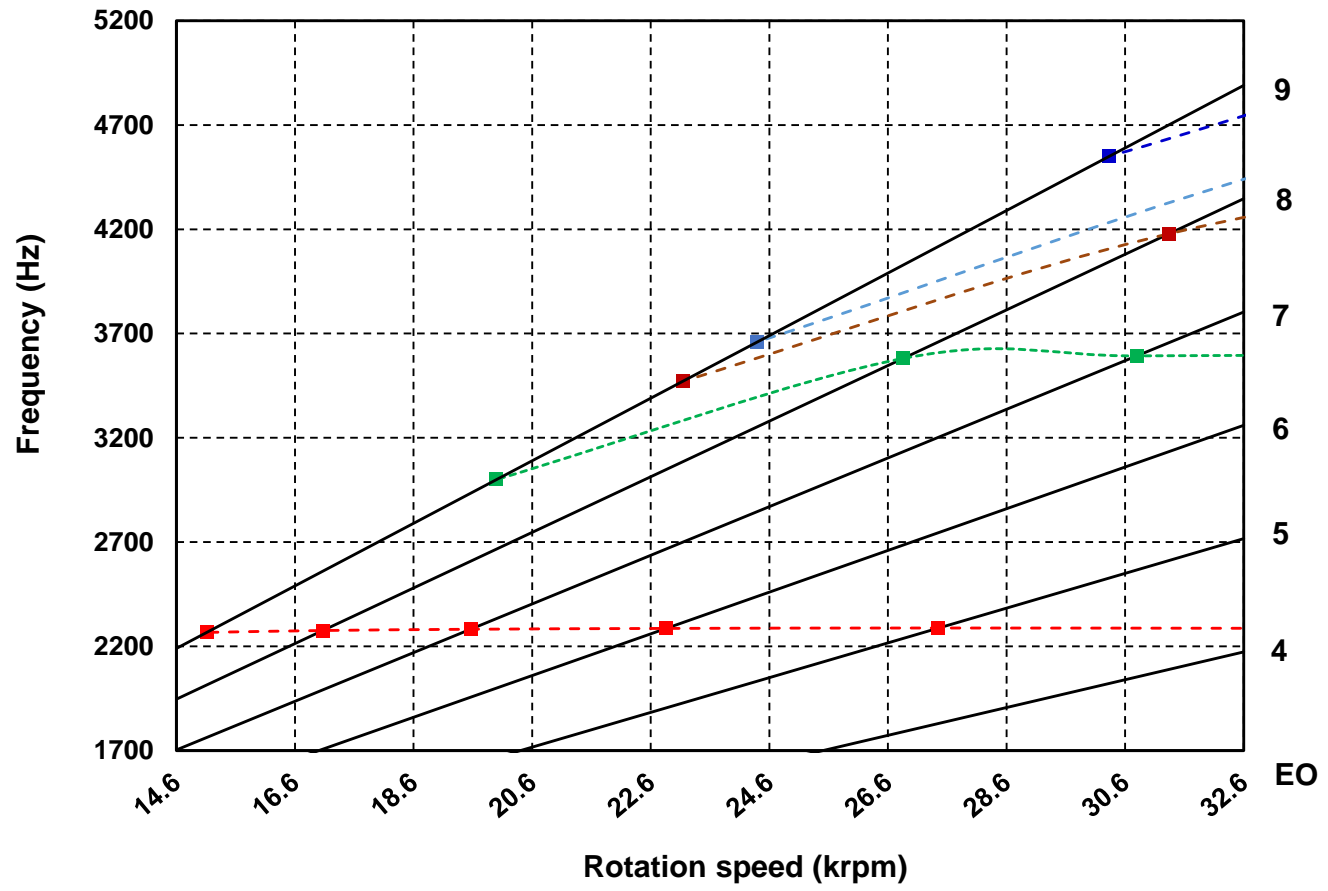

Figure 7.26: Campell diagram for the redesigned full impeller wheel 


\section{DESIGN AND ANALYSIS OF A NEW TRANSONIC CENTRIFUGAL IMPELLER}

this section presents the CFD analysis of the redesigned impeller at the design point. It is essential to verify that the TD1 calculations have good accuracy when compared with CFD calculations.

Figures 7.27, 7.28 and 7.29 compare the blade surface pressure calculated by the inviscid TD1 code and viscous solver CFX. It can be confirmed that the matching between the inviscid and viscous solutions is quite good. We can conclude that:

- Near the blade hub, as is shown in Fig. 7.27a and 7.27b, the predicted pressure distribution matched almost everywhere in the streamwise direction on the both full and splitter blade surface.

- At $50 \%$ of the spanwise height, shown in Figs. 7.28a and 7.28b, one can see that the matching is still quite good but not the same level of the hub loading. Particularly, a negative pressure peak at the leading edge of the suction surface is not captured in the TD1 calculations. Such a drastic change of pressure indicates the presence of shock waves, i.e., the shock waves appear at the midspan of the blade in the vicinity of the leading edge. Again, this shock wave appearance cannot be captured in TD1. Another interesting phenomenon to note is the local "pressure bump" on the pressure surface near the trailing edge predicted in CFX. This indicates that there can be a rapid velocity variation in the region which is likely to induce flow separations. It will be confirmed later when the flow aerodynamics is presented.

- Near the blade shroud, as are shown in Figs. 7.29a and 7.29b, the two predictions have larger differences comparing hub and midspan section, which is due to the tip leakage effect modelled in CFX but not in TD1. However the general trend still has quite good agreement. In addition to the negative pressure peak observed at the full blade leading edge, such a pressure jump is also seen on the splitter blade's leading edge. It is worth mentioning that matching of the static pressure distribution on the suction surface is much better comparing with the pressure surface for both full and splitter blade. This is because the flow in one blade channel can slip from the pressure 


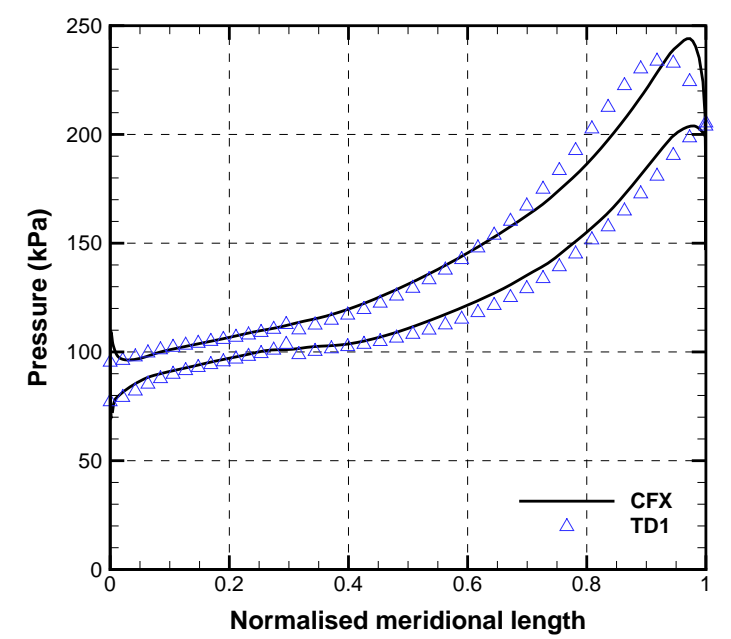

(a) Near the full blade hub

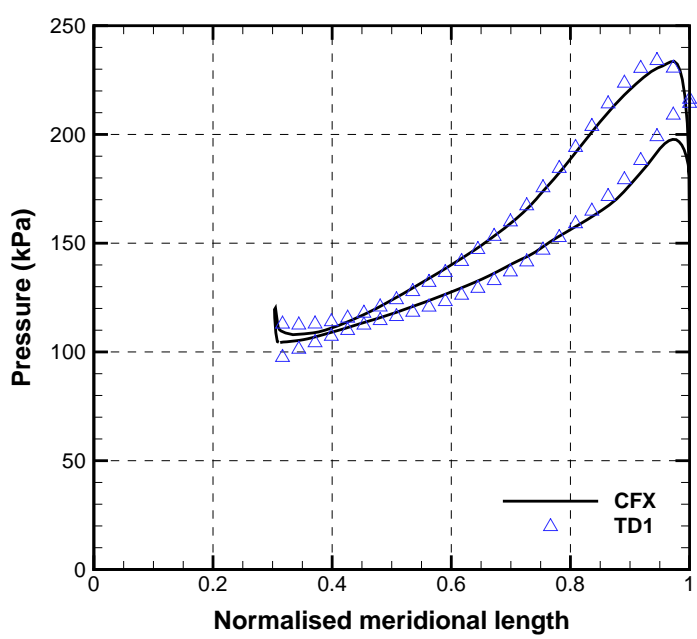

(b) Near the splitter blade hub

Figure 7.27: Comparisons of blade loading calculated in CFX and TD1 near the hub (5\% spanwise height)

surface and cross the tip gap entering the adjacent channel. The other important feature that should be noted is the "dip" on the CFX prediction of the upper surface's static pressure distribution near the place where the splitter blade leading edge is positioned and strong blade loading is also present there, shown in Fig. 7.29b, i.e., there can be strong flow interactions in this region. This is also a feature that cannot be predicted by the inviscid code.

In Fig. 7.30 the pressure contour on the entire impeller surface is compared between the CFD and TD1 calculations. In general, although there are some differences due to the viscosity and tip gap effects, TD1 still has fairly good agreement with the CFX solutions and the inviscid solution can revel quite a bit of flow physics on the blade surface. This validation process is very essential since the inverse design method is a flow-physics oriented approach. If the prescribed blade loading are too different from the viscous calculations, the designs will not be reliable. In the following section, we shall compare the off-design point's performances between the baseline and redesigned impeller. 


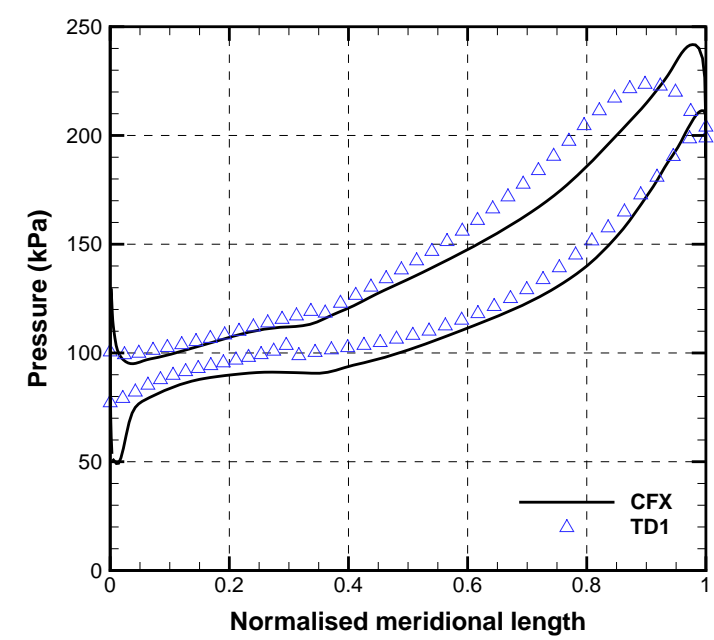

(a) Full blade midspan

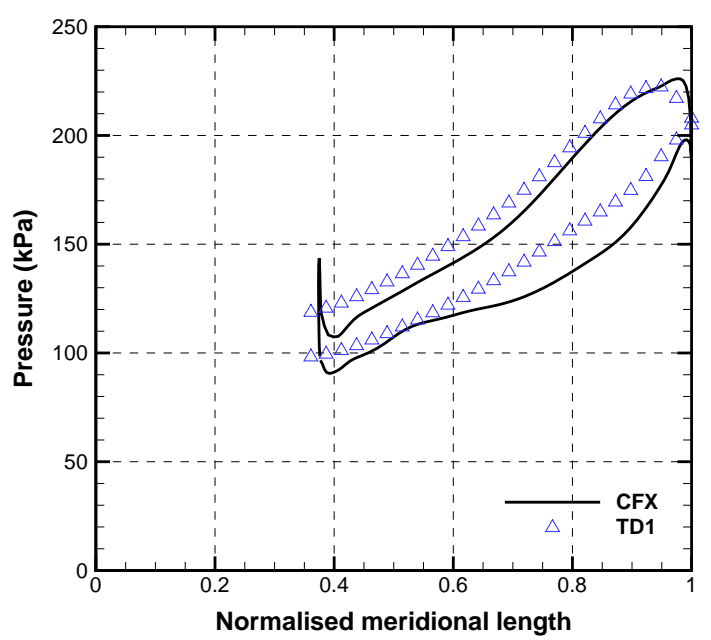

(b) Splitter blade midspan

Figure 7.28: Comparisons of blade loading calculated in CFX and TD1 at the midspan (50\% spanwise height)

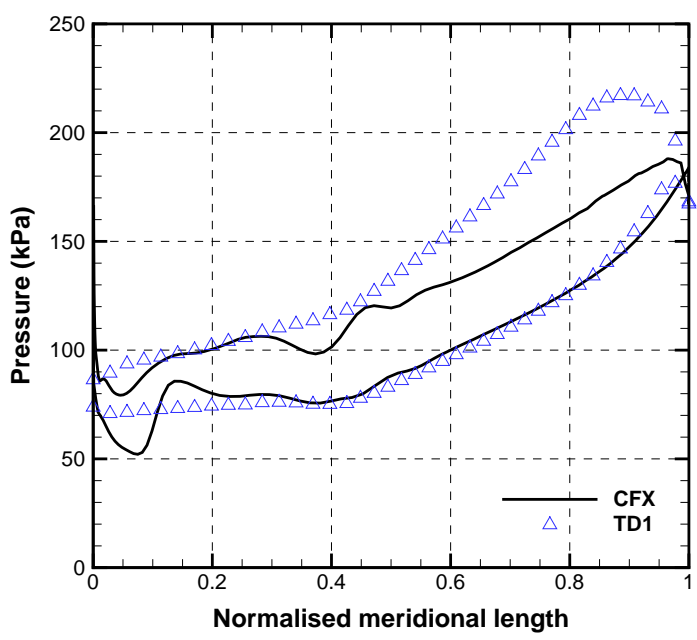

(a) Near the full blade shroud

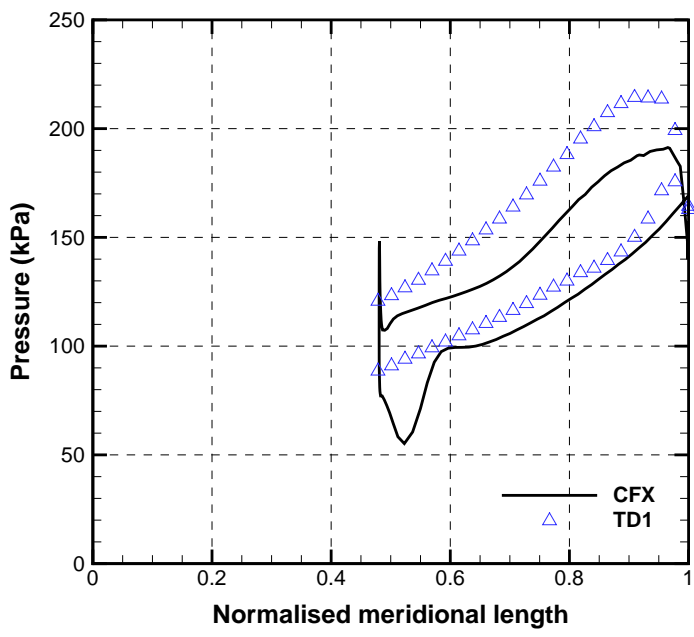

(b) Nnear the splitter blade shroud

Figure 7.29: Comparisons of blade loading calculated in CFX and TD1 near the blade shroud (90\% spanwise height) 


\section{DESIGN AND ANALYSIS OF A NEW TRANSONIC CENTRIFUGAL IMPELLER}

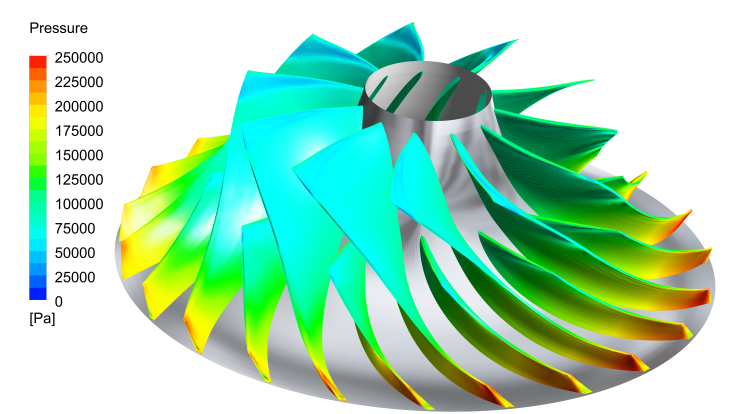

(a) Blade surface pressure contour calcluated in CFX

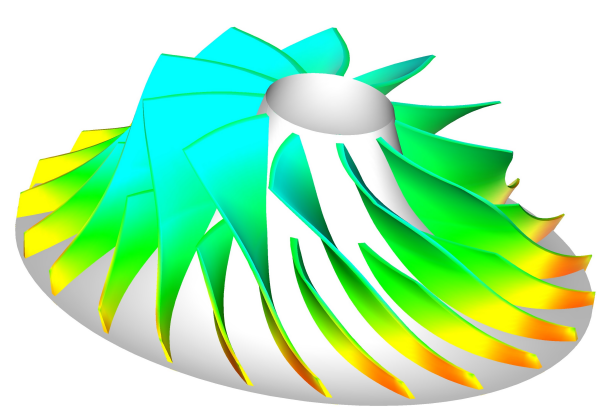

(b) Blade surface pressure contour calculated in TD1

Figure 7.30: Comparisons of the blade surface pressure calculations between inviscid TD1 and viscous CFX solver

\subsection{Performance comparisons at the peak speed}

In this section, the flow details of the two impellers operating at the BEP (best efficiency point) of speedline 1800(30krpm) will be analysed and compared. Following that, the acoustic calculations at the near field for both designs will be performed and the noise spectrum compared.

\subsubsection{Internal flow field details}

As the final blade loading prescribed in TD1 is driven by the main target of reducing the noise, reduction of the shock strength on the blade is of primary concern in the design iterations. In Figs.7.31-7.33 the Mach number distribution at different spanwise heights are compared between the redesigned and original impeller.

- Near the hub section, a very noticeable reduction of shock strength can be found on the LE.

- At the midspan shown in Fig. 7.32, a normal shock at the suction surface of both designs is clearly visible. In the downstream of this normal shock, the flow behaviours are quite distinct between the two designs. A street of supersonic flows appears on the suction surface of the baseline full blade and it almost extends to the trailing edge. This phenomenon cannot be 


\section{DESIGN AND ANALYSIS OF A NEW TRANSONIC CENTRIFUGAL IMPELLER}

found on the suction surface of the redesigned full blade. It is also worth noticing that a low momentum zone has appeared on the suction surface of baseline splitter near the trailing edge, while it is not found on the redesign.

- A detached bow shock is formed at both designs' full blade leading edge shown in Fig. 7.33. A channel shock is also formed in the baseline impeller, which starts from the leading edge of the pressure surface and hits the adjacent suction surface. Additionally, a very obvious low momentum zone is found on the baseline splitter's both suction and pressure side, whereas this phenomenon on the redesign is much less obvious, which implies the reduction of the tip leakage flows on the redesign.

In Figs. 7.34-7.36 the static pressure distributions on the full blade are compared at three different locations. It can be seen that the redesigned impeller is loaded much less at inducer while more loaded on the exducer, which conforms to the aft-loading prescriptions in TD1. In this case, unloading the inducer is quite important for reducing the impeller noise, which will be elucidated with acoustic calculations in the next subsection.

Figure 7.37 compares the exit flow close to the impeller trailing edge of two designs. Obviously, the inversely designed impeller has a much more uniform exit flow pattern. A big low momentum zone initiated from the splitter's suction side of the baseline is greatly removed on the redesign. This should result in significant fewer mixing losses in the vaned diffuser and hence improve the stage performances, and it shall be confirmed by the compressor maps presented in the next section.

\subsubsection{Near field acoustics: numerical calculations}

In this subsection the numerical calculations of the acoustic field at the near field will be presented. Figure 7.38 shows the schematic view of the idealised relative position between the observer and impeller, which is very similar to Fig. 5.16 used in Chapter 5 for the SRV2 impeller. In the real test the microphones were placed at the inlet of a very long intake duct due to the facility limit, and it will be shown in the next chapter. 

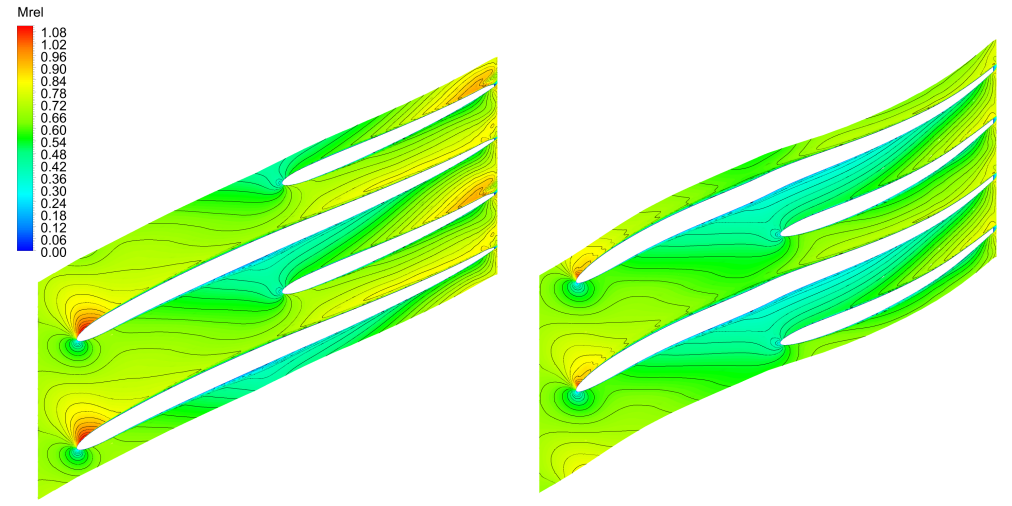

Figure 7.31: Relative Mach number comparisons between baseline (left) and redesign (right) near the hub (10\% spanwise height) at speedline 1800, BEP
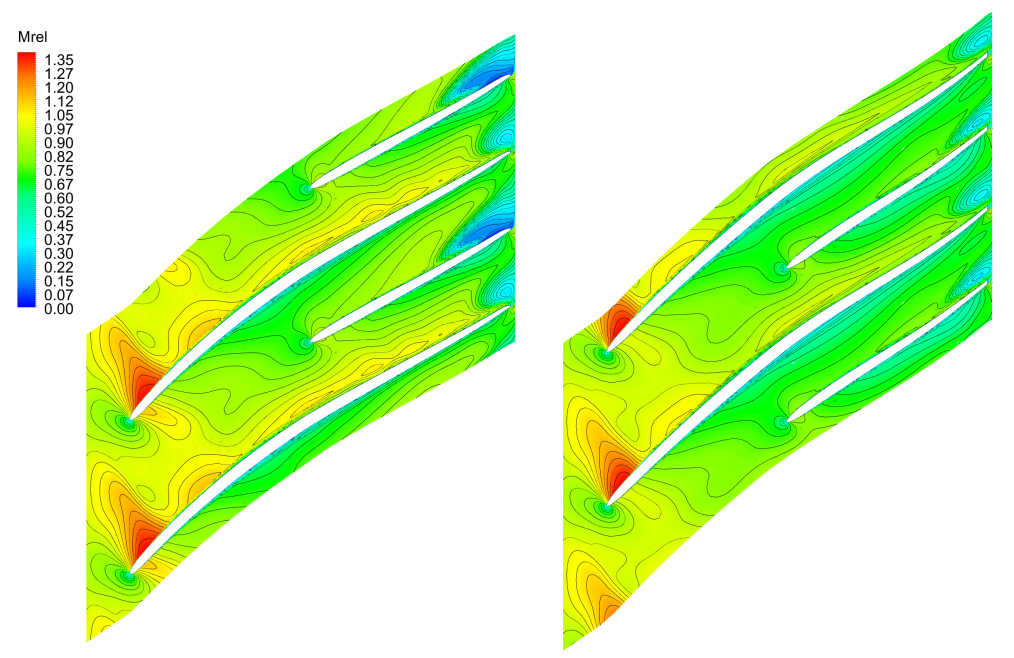

Figure 7.32: Relative Mach number comparisons between baseline (left) and redesign (right) at the midspan (50\% spanwise height) at speedline 1800, BEP 


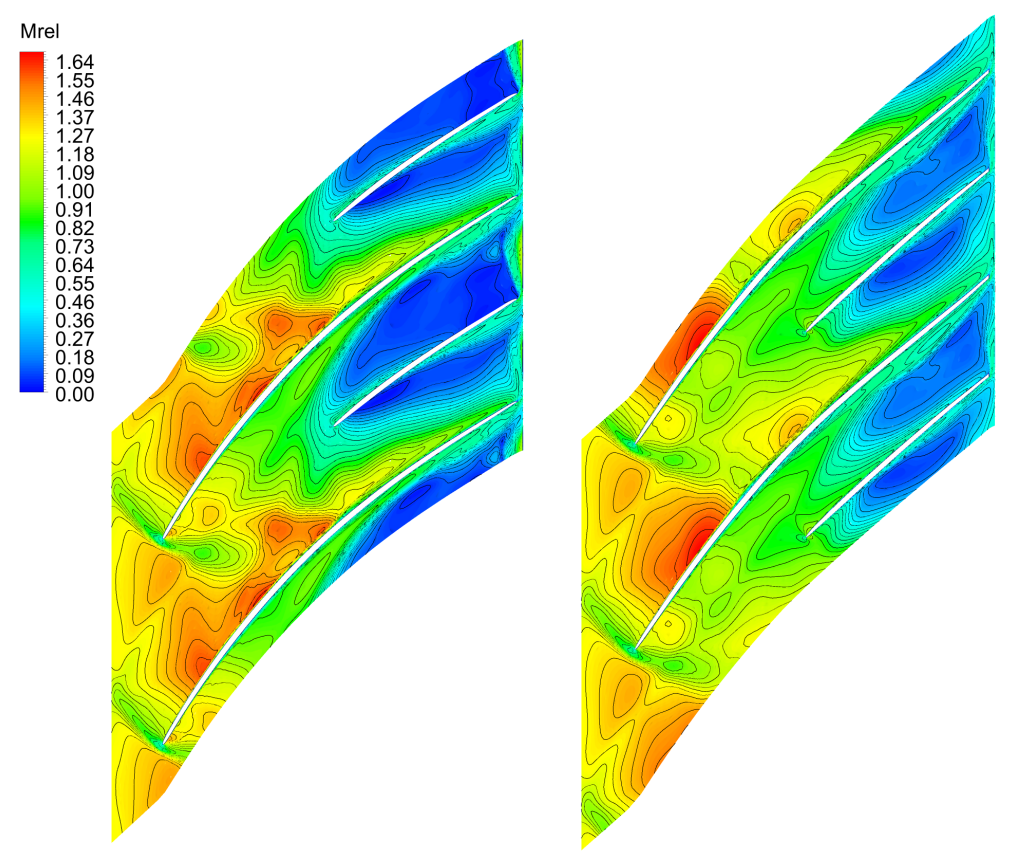

Figure 7.33: Relative Mach number comparisons between baseline (left) and redesign (right) near the shroud (90\% spanwise height) at speedline 1800, BEP

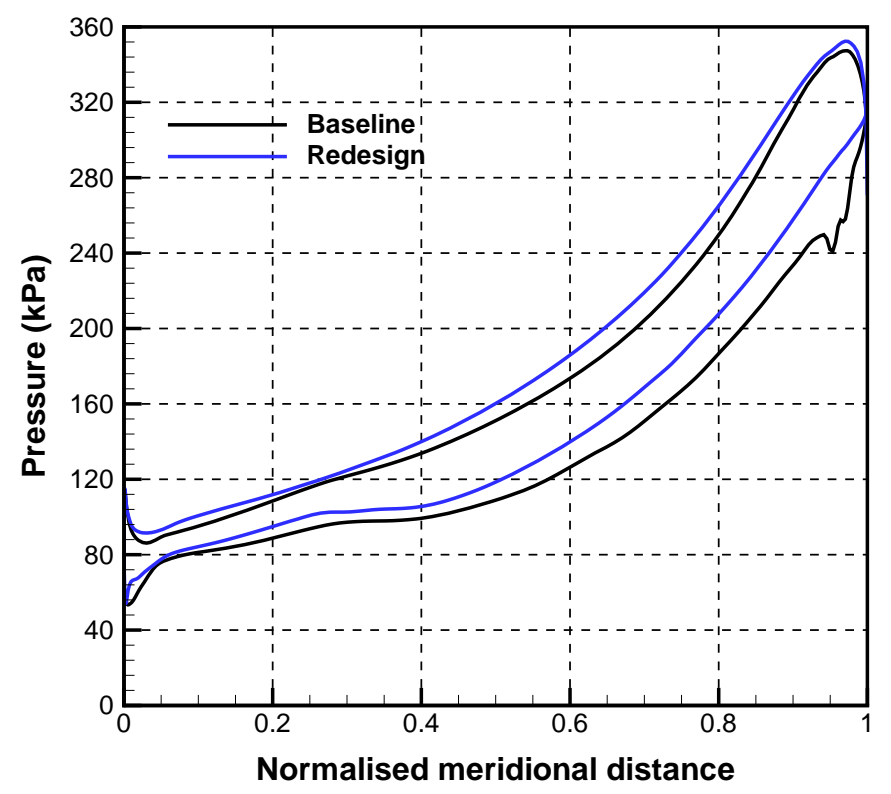

Figure 7.34: Blade surface pressure comparisons between baseline (left) and redesign (right) near the hub (10\% spanwise height) at speedline 1800, BEP 


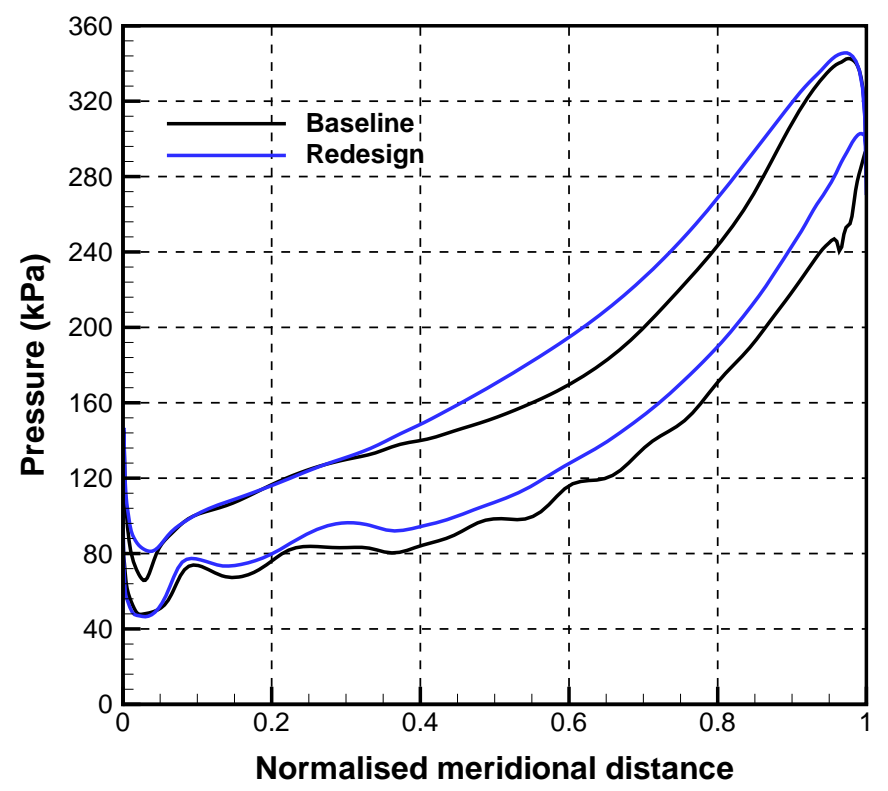

Figure 7.35: Blade surface pressure comparisons between baseline (left) and redesign (right) at midspan (50\% spanwise height) at speedline 1800, BEP

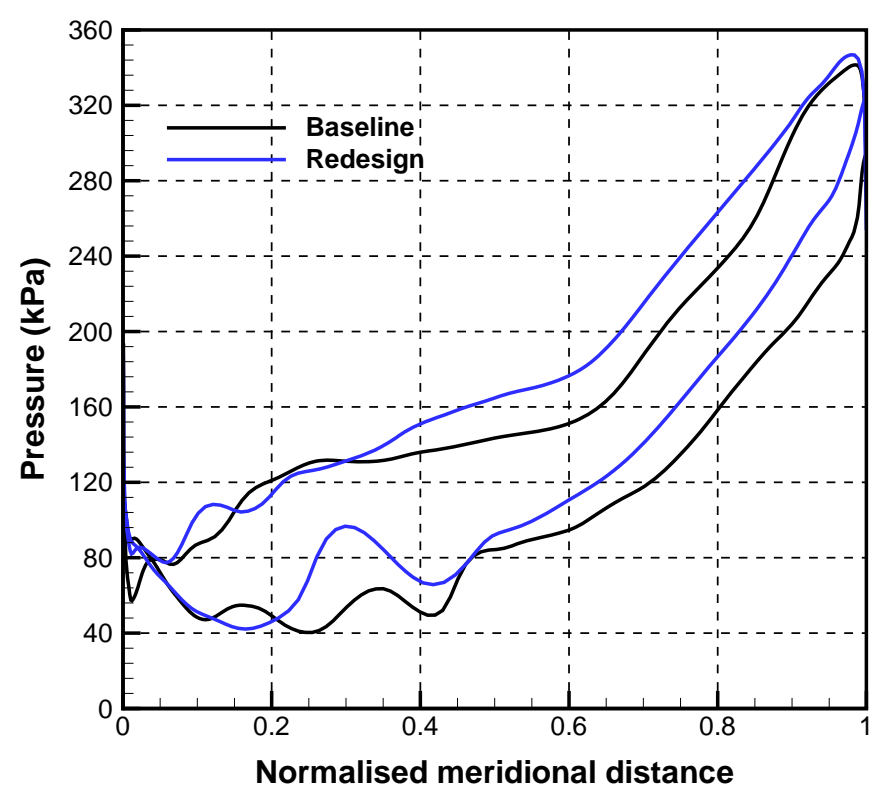

Figure 7.36: Blade surface pressure comparisons between baseline (left) and redesign (right) near the shroud (90\% spanwise height) at speedline 1800, BEP 


\section{DESIGN AND ANALYSIS OF A NEW TRANSONIC CENTRIFUGAL IMPELLER}

\section{Mrel}

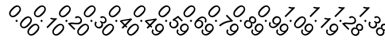
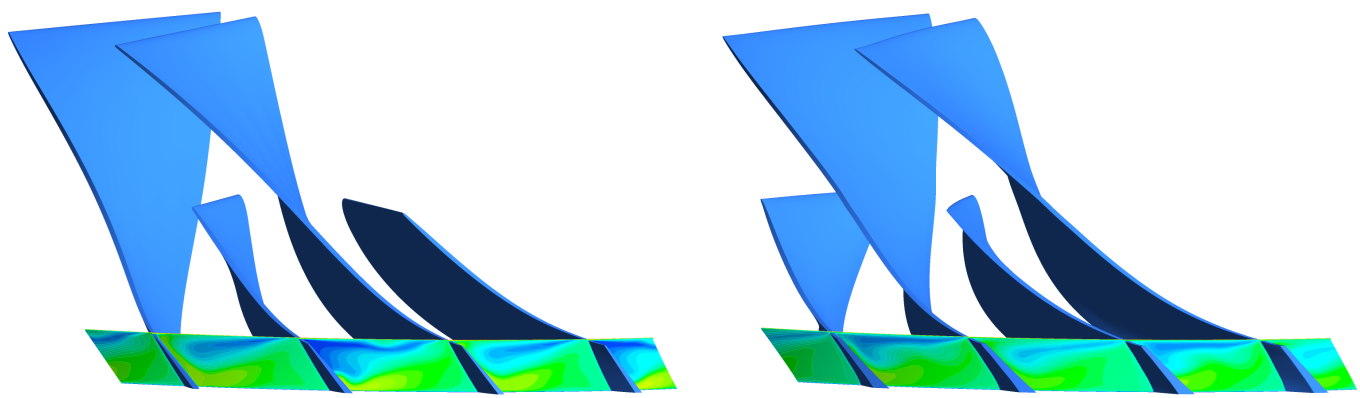

Figure 7.37: Relative Mach number comparisons between baseline (left) and redesign (right) near the impeller exit at speedline 1800, BEP

As has been previously explained, there is no duct effect on the acoustic propagation considered in our acoustic prediction model. Our very intention is rather to reduce the noise at the source, and the acoustic prediction is therefore set up in the manner that is similar to the SRV2 compressor noise measurements elaborated in Chapter 5, i.e., the sound predictions are performed at those locations that are close to the impeller leading edge.

It is assumed that 4 microphones are located 90 degrees to each other in the inner wall of the duct whose inner diameter is $50 \mathrm{~mm}$ and those microphones are $240 \mathrm{~mm}$ away from the impeller LE in the axial direction, as can be seen in Fig. 7.38 .

Figure 7.39 shows the results of acoustic components computed using the solver developed in Chapter 3 for both impellers operating near choke condition. As expected one can see that the loading noise is a much more important source than the thickness noise. Comparing Figs. 7.39a and 7.39b one can see that the redesigned impeller blade induces larger thickness noise whereas smaller loading noise. Figure 7.40 shows that the resultant noise from all blades, which are interpolated from the data in Fig. 7.39c. Figure 7.41 compares the noise levels from both designs in the frequency domain. One can see that the blade passing tones are clearly visible in both cases, and the tone of the redesigned impeller at the 1st harmonic of the blade passing frequency is about $3.41 \mathrm{~dB}$ lower than 


\section{DESIGN AND ANALYSIS OF A NEW TRANSONIC CENTRIFUGAL IMPELLER}

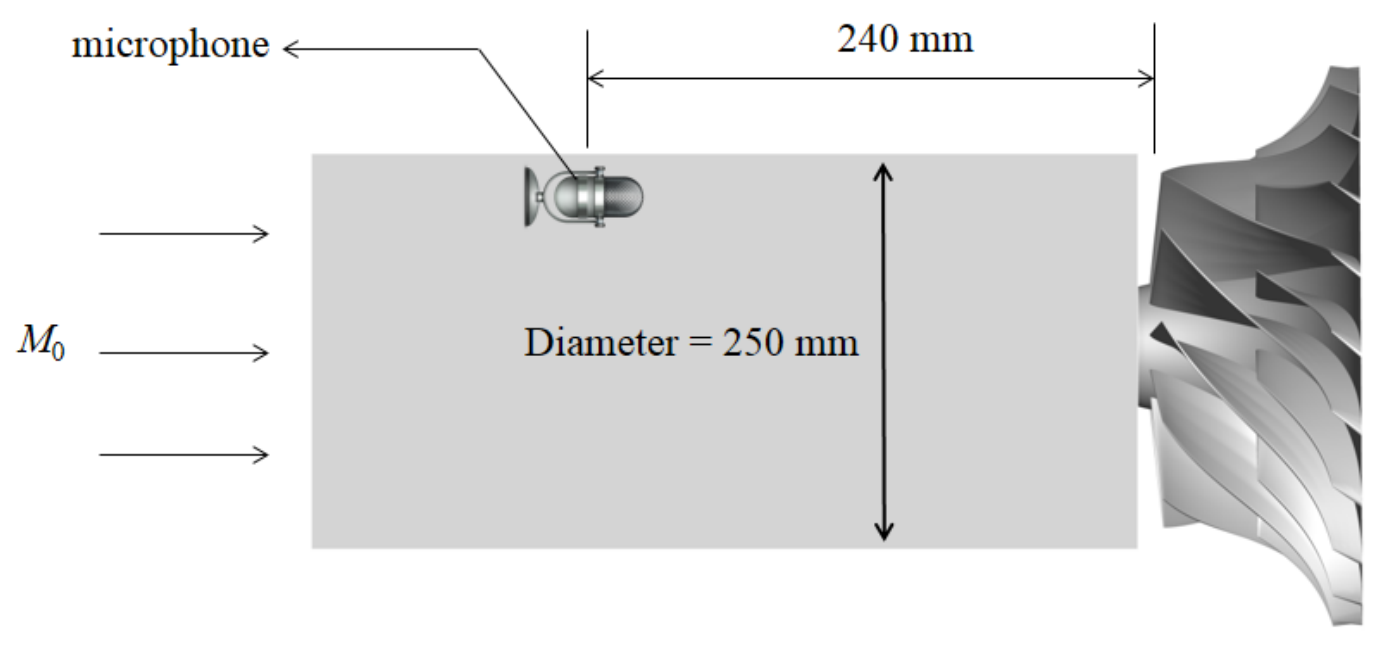

Figure 7.38: Relative positions between the impeller and microphones for numerical calculations

the baseline. Figures 7.42a to 7.44 present the comparisons for both impeller operating near the BEP. It should be noted that the advantages at this point of the redesign are not as good as them at the choke condition. Figure 7.44 shows that the tone at the 1st BPF has only about $1 \mathrm{~dB}$ advantages.

\subsubsection{Summary}

In this section we presented some flow physics on the baseline and redesigned blade channel. The Mach number contours and blade surface pressure distributions clearly showed that the redesigned impeller has much reduced loading on the inducer and hence reduced the strength of shocks on the inducer. The acoustic calculations confirmed that the inversely designed blade can produce over $3 \mathrm{~dB}$ less noise at the maximum flow conditions and $1 \mathrm{~dB}$ at the BEP. The experimental measurements of the sound levels will be presented in the next chapter. 


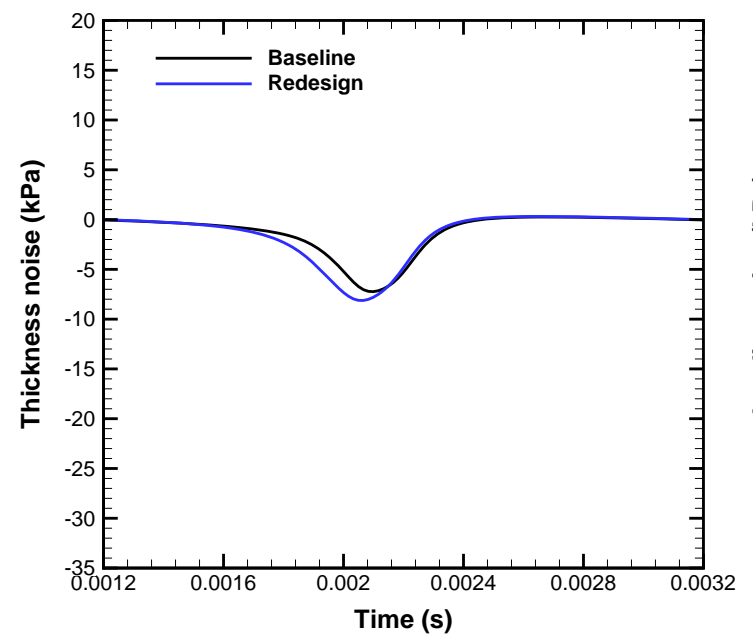

(a) Thickness noise

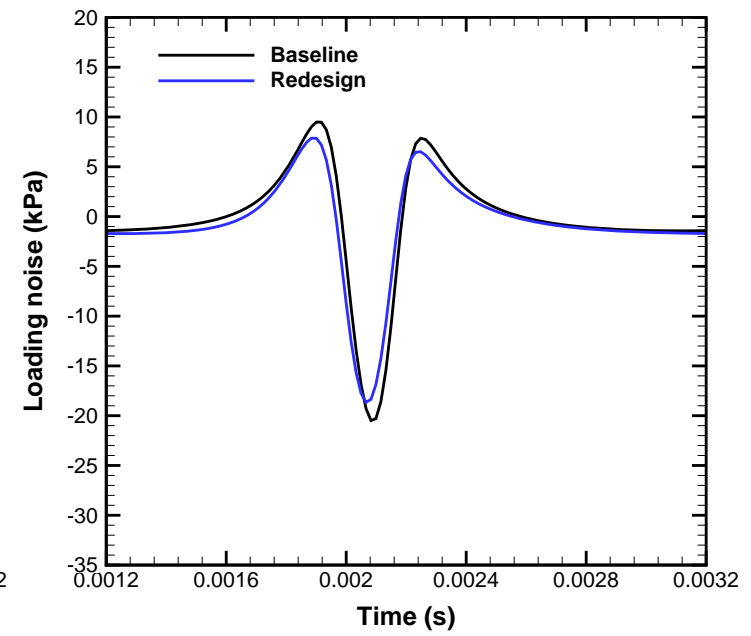

(b) Loading noise

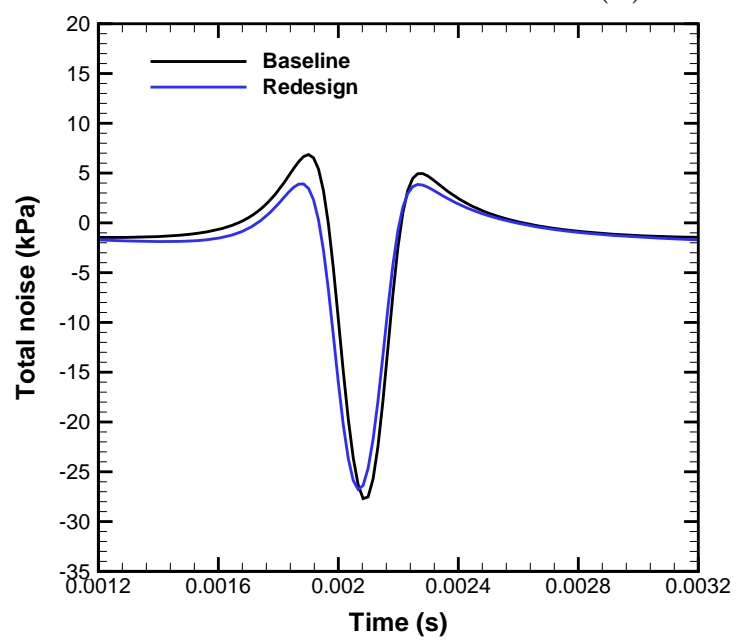

(c) Total noise

Figure 7.39: Comparisons of the acoustic pressure signature in one period between the baseline and redesigned impeller near the choke: single blade 


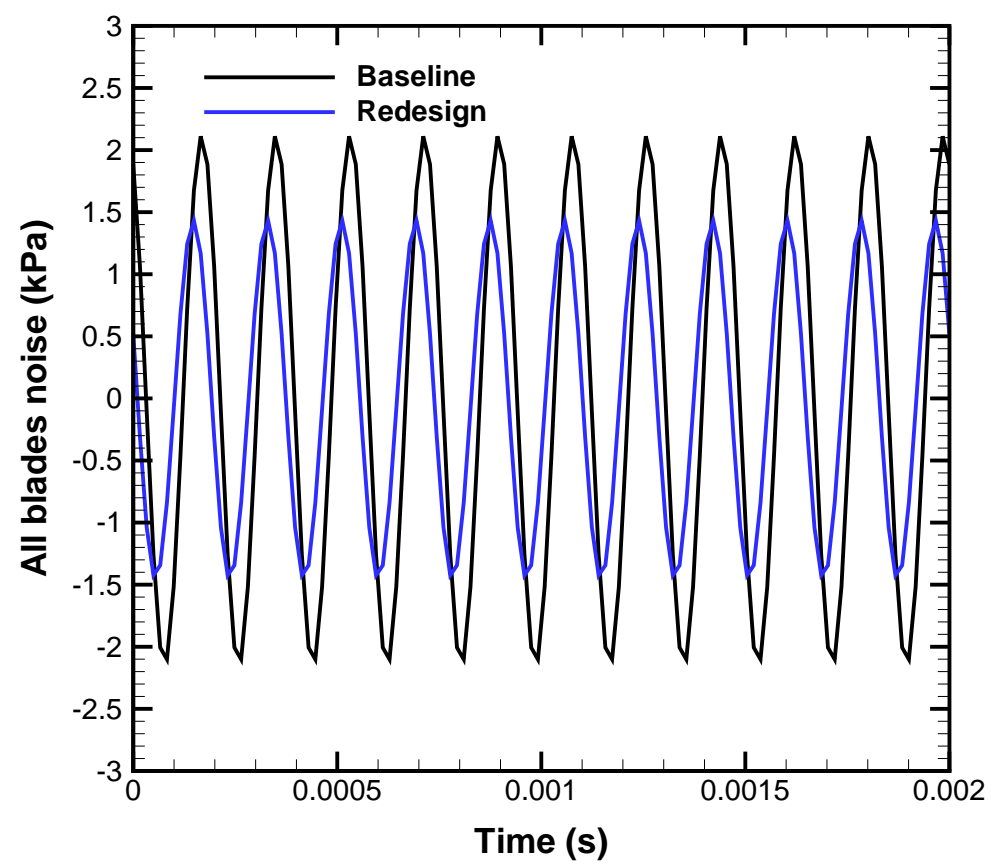

Figure 7.40: Comparisons of the total acoustic pressure signature in one period between the baseline and redesigned impeller wheels near the choke: all blades

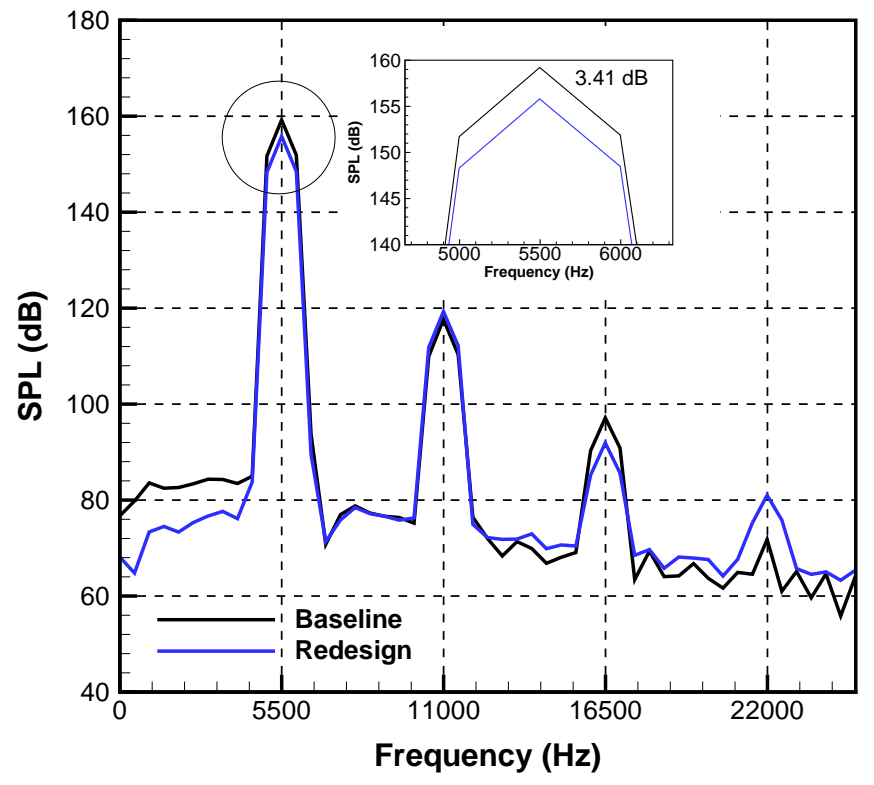

Figure 7.41: Comparisons of sound pressure level in the frequency domain between baseline and redesign near choke 


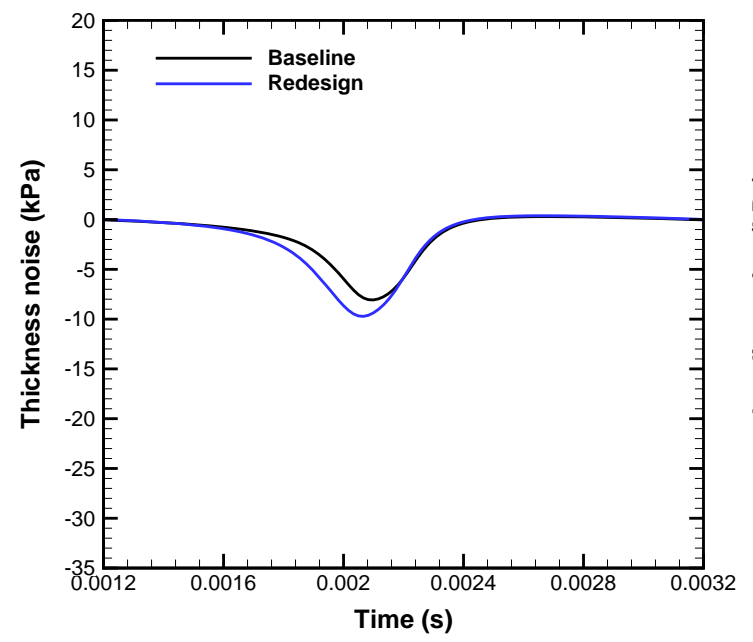

(a) Thickness noise

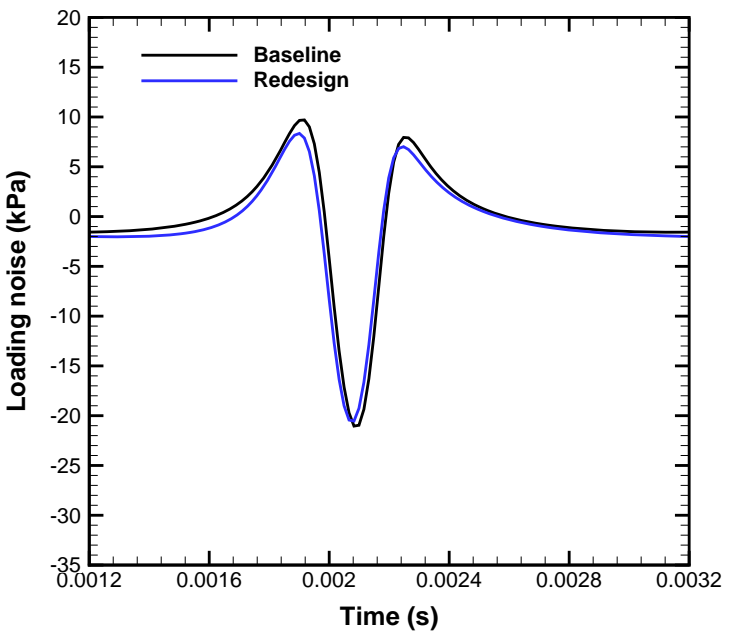

(b) Loading noise

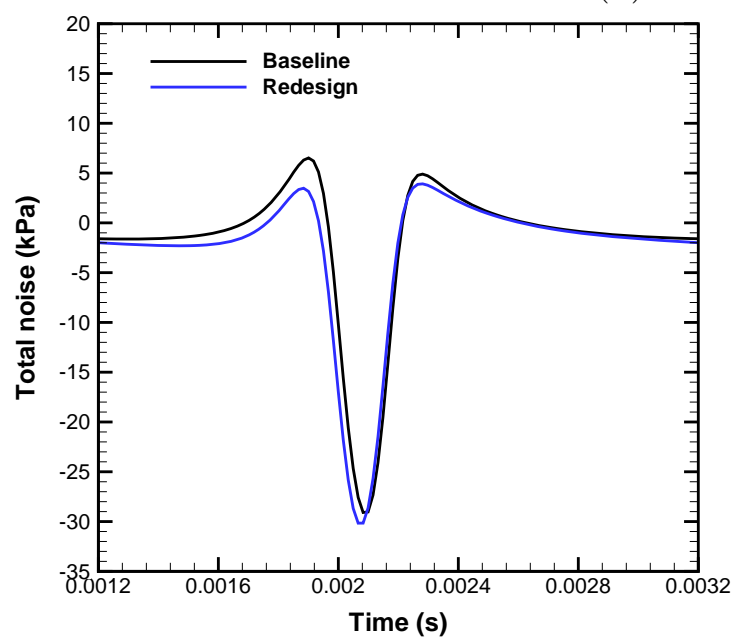

(c) Total noise

Figure 7.42: Comparisons of the acoustic pressure signature in one period between the baseline and redesigned impeller near the BEP: single blade 


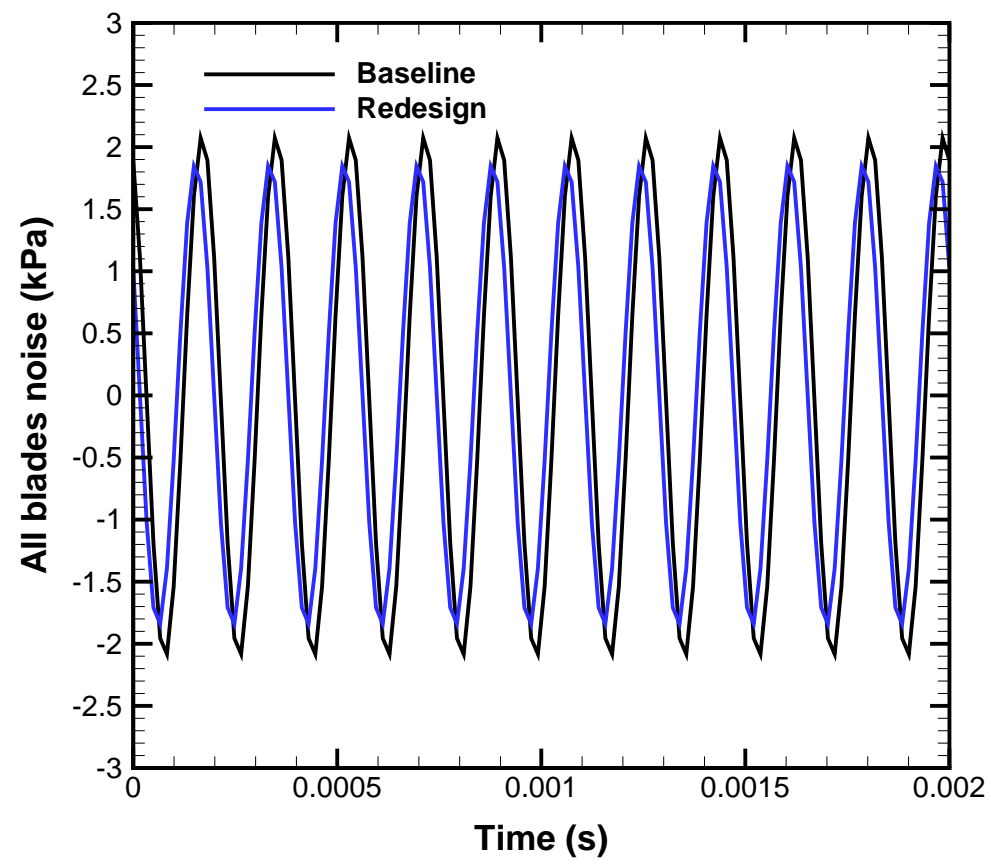

Figure 7.43: Comparisons of the total acoustic pressure signature in one period between the baseline and redesigned impeller wheels near the BEP: all blades

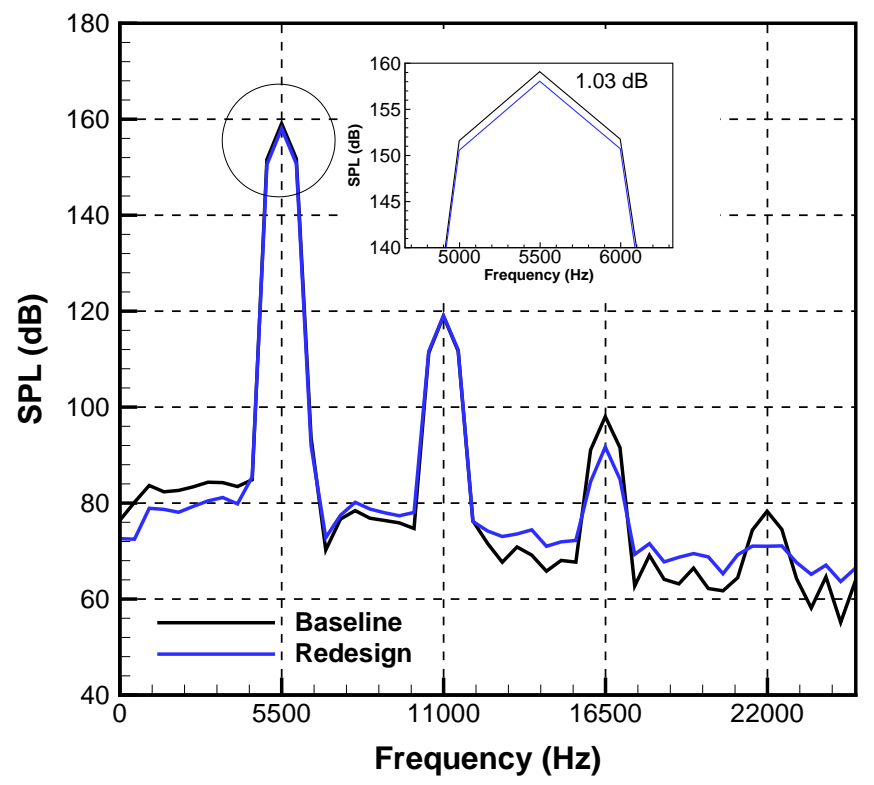

Figure 7.44: Comparisons of sound pressure level in the frequency domain between baseline and redesign near the BEP 


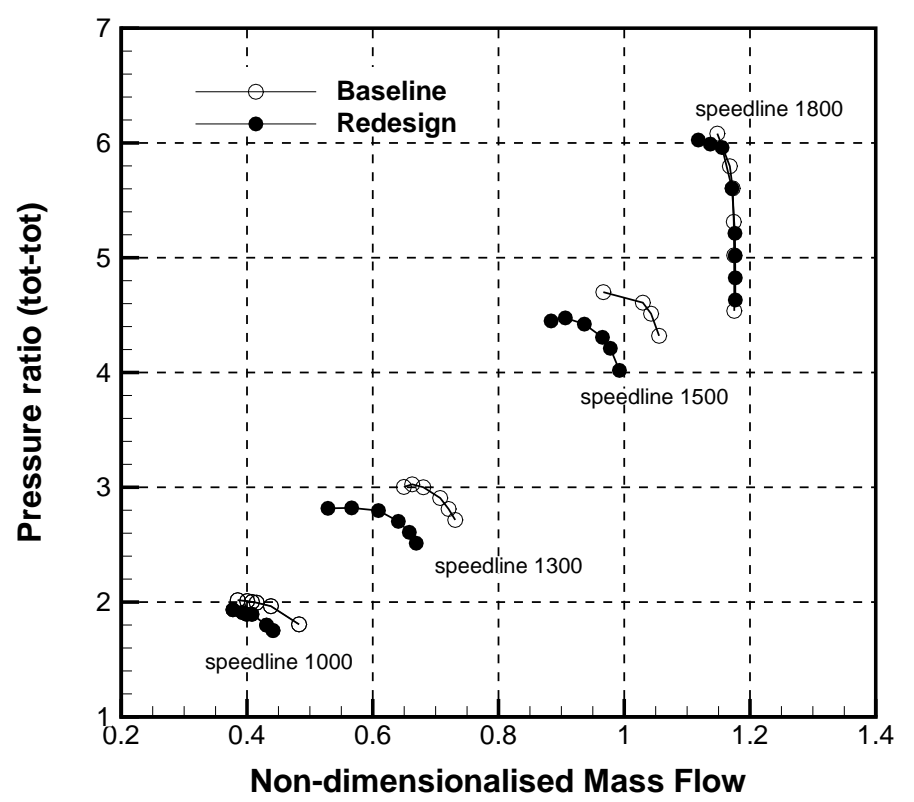

Figure 7.45: Comparisons of the predicted pressure ratio between baseline and redesign at multiple speedlines

\subsection{Predicted performances of the complete stage of the redesign}

In this section, we will present the results of the full stage simulations for the redesigned compressor, including the impeller, vaned diffuser and volute, at the same speedlines as the baseline. The CFD model used was set up exactly the same as that used for the previously mentioned baseline compressor shown in Fig. 7.6. From Figs. 7.45 and 7.46 it can be seen that:

- the redesigned compressor has the very close pressure ratio and choke margin at the primary speedline 1800 while the surge margin is improved;

- there is a very big improvement of the stage efficiency at the top speed. The peak efficiency has been increased by over $4 \%$;

- the pressure ratio and efficiency at the lowest speed 1000 of the two designs are quite close but the redesign has a smaller choke margin;

- the choke margins and pressure ratios of the redesign at speedline line 1300 


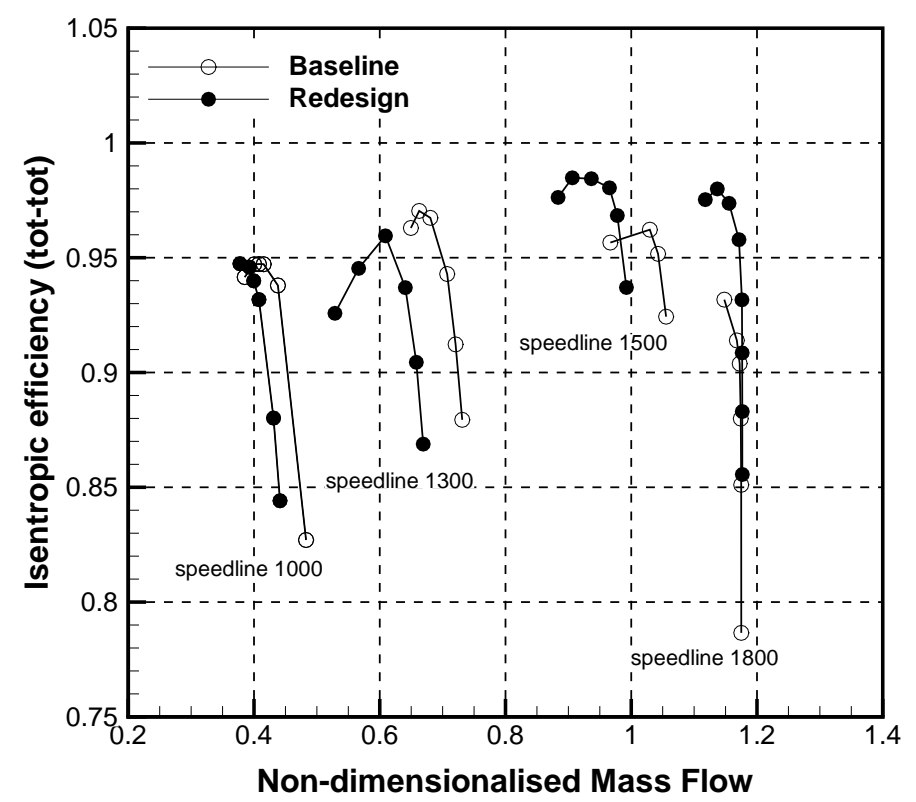

Figure 7.46: Comparisons of the predicted isentropic efficiency between baseline and redesign at multiple speedlines

and 1600 are also less than the baseline, and the possible reasons will be explained in the next chapter with more detailed flow field analysis;

- the global peak efficiency of the compressor map has been shifted from speedline 1300 of the baseline to 1600 of the redesign.

\subsection{Summary}

In this chapter, CFD calculations were first attempted for the baseline compressor stage. The predicted characteristics were compared with the experimental measurements at design and off-design speeds. Reasonably good agreement was obtained, as can be seen in Figs. 7.7 and 7.8. The CFD model was therefore considered acceptable for any further stage performance predictions.

The detailed procedures of designing a new impeller using TURBOdesign1 code were subsequently presented. Fairly detailed explanations for each design step were given, and a loading-prescription strategy has also been discussed for a transonic centrifugal impeller. The inversely designed impeller was evaluated 


\section{DESIGN AND ANALYSIS OF A NEW TRANSONIC CENTRIFUGAL IMPELLER}

using FEA first to make sure its structure integrity satisfies Napier's requirement. The simplified and Napier's internal modelling both confirmed that the redesigned impeller has smaller stress concentration, less deflection, and higher vibration frequency than the baseline design.

In order to explain the efficiency gain of the redesigned impeller, some internal flow details were compared. It has been shown that the redesigned impeller gives a weaker shock strength at the leading edge, which is quite beneficial for the aerodynamic efficiency and aerodynamic noise reductions.

As expected from the internal flow analysis, the reduced shock strength at the leading edge did give rise to less noise. The near field acoustic calculations showed that there can be $3 \mathrm{~dB}$ less noise at maximum flow condition and $1 \mathrm{~dB}$ less at the BEP. Even though these noise predictions were performed close to the impeller where no test data can be taken, the results provided evidence that the noise has been reduced from the source. In the next chapter, the actual acoustic measurements of two designs will be presented.

At the end, detailed comparisons of the predicted compressor characteristics at multiple speeds between two designs were presented. It can be seen from Fig. 7.46 the redesigned compressor has significant advantages of surge margins and efficiency at the higher speeds, which are exactly required by the design targets. However, it appears to be some reduction of choke margins, which will be explained in the following chapter. 
"Experience never errs; it is only your judgements that err by promising themselves effects such as are not caused by your experiments."

Leonardo da Vinci (1452 - 1519)

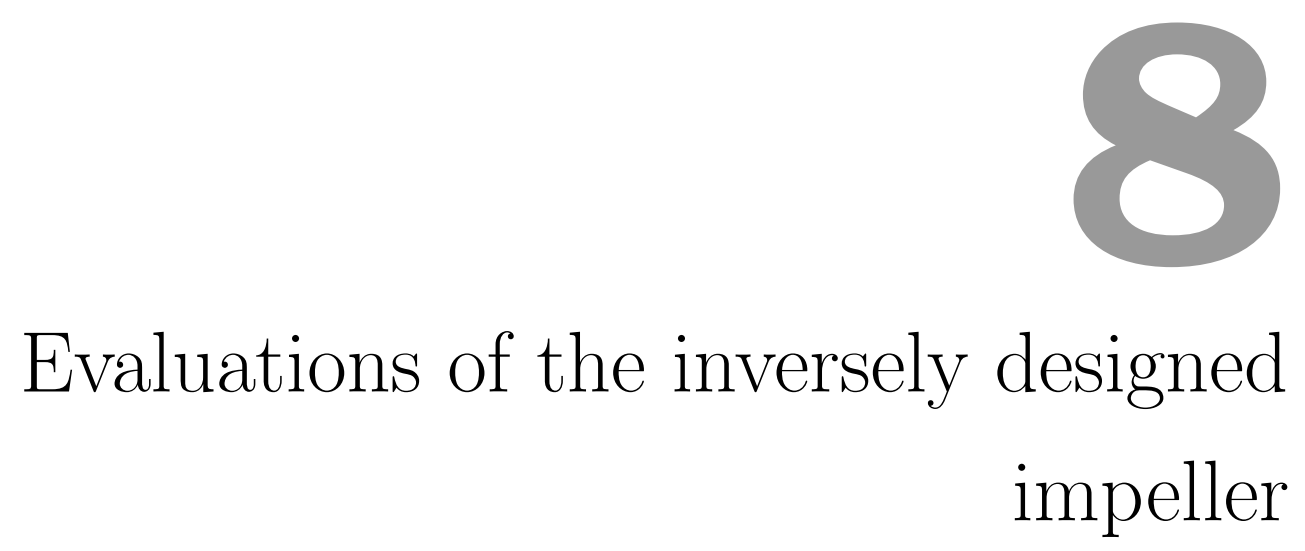

In this chapter, we present the evaluations of the redesigned impeller by experimental measurements of its performance map and acoustic levels. All the results will be compared with the original design commensurably. It will be demonstrated that

- the CFD predictions for the stage performance in the last chapter are in good agreement with the test data;

- the redesigned impeller has improved both aerodynamic and aeroacoustic performance at the nominal speeds

These performance improvements will be explained with detailed comparisons of flow field from CFD calculations at different operating points. 


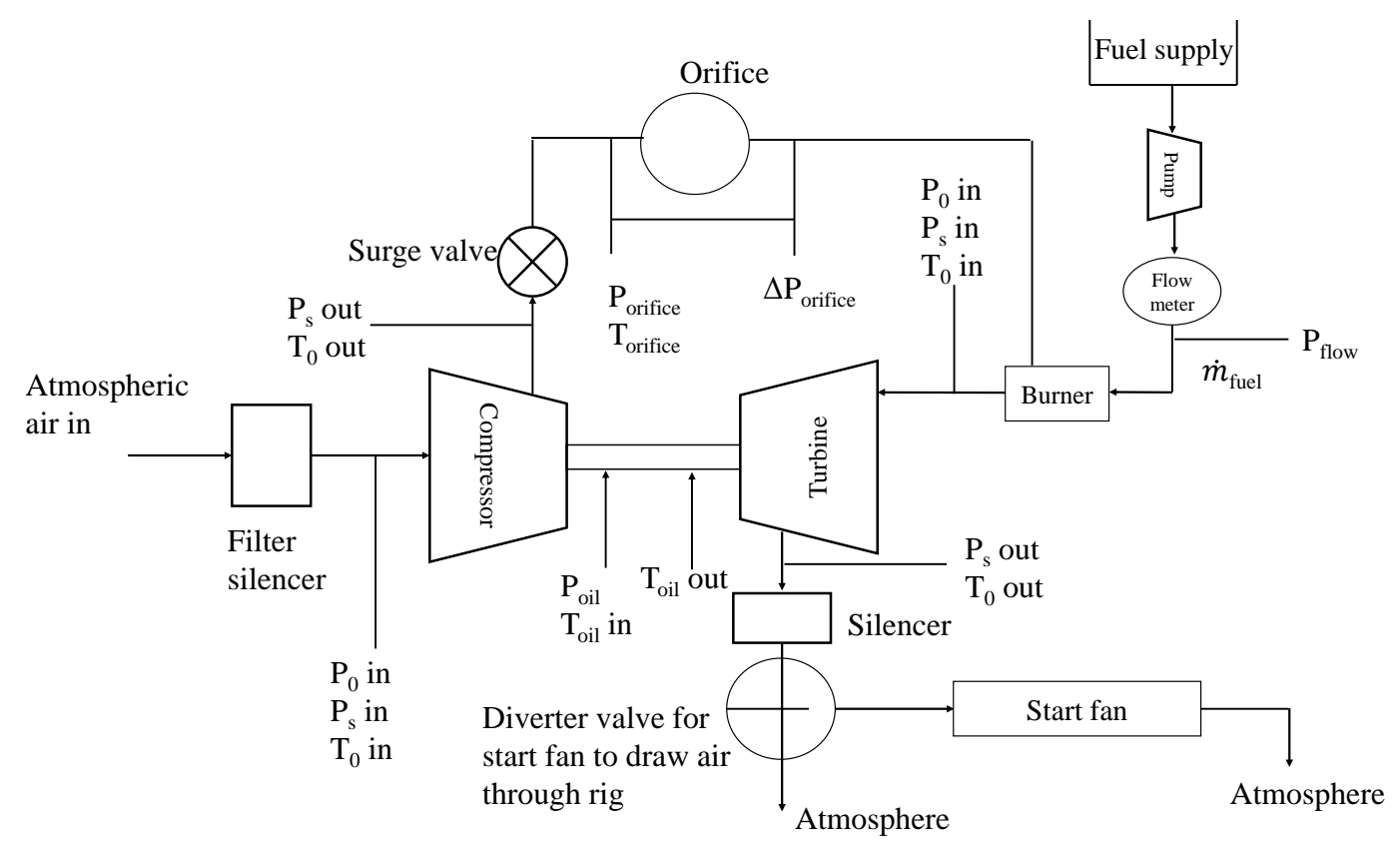

Figure 8.1: Schematic of turbocharger control facility

\subsection{Test rig}

All the compressor tests were completed at Napier Turbochargers Ltd, and the layouts of the turbocharger and oil controlling system are shown in Figs. 8.1 and 8.2. One can see that the atmospheric air is first drawn into the compressor, and the temperature and pressure are measured at the compressor inlet. A surge valve is used to control the incoming flow capacity so as to adjust the pressure ratio. A fuel supply tank, pump and flow meter are used to control the fuel flow rate, and the compressed air will be delivered to the burner and mixed with the fuel for combustion. The exhausted gas will be used to drive an axial turbine, which in turn spins the compressor through a shaft.

\subsection{Validation of CFD predictions}

In Figs. 8.3 and 8.4, the CFD predictions of the redesigned compressor stage performance are compared with the measurements at variable speedlines in the same manner as the baseline design. As one can see, the pressure ratio predictions 


\section{EVALUATIONS OF THE INVERSELY DESIGNED IMPELLER}

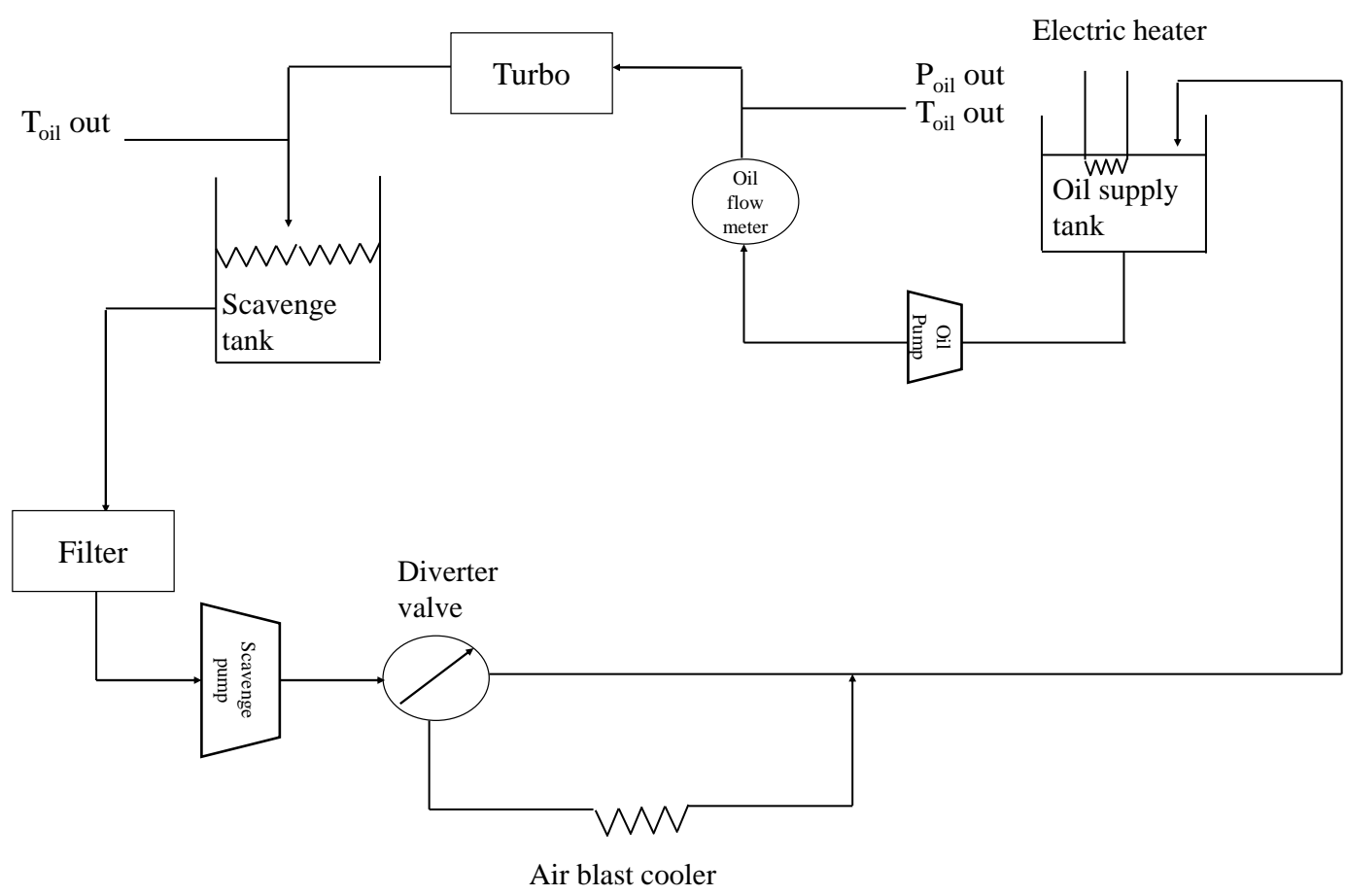

Figure 8.2: Schematic of oil control facility

are in reasonably good agreement with the test data at different speedlines. The predicted choke margins at speedline 1000, 1300 and 1600 match the test data fairly well. However, the CFD calculation of the choke margin at the highest speed under-estimates the choke margin when compared with the test data, which is likely due to the exclusion of the ported shroud in the CFD simulations. In the work by Chen \& Lei [2013], they also showed that inclusion of a ported shroud can increase the choke margin at the highest speed while having few effects on the lower rpms. Further to that, the predicted surge margins are smaller than the experimental data at speedline 1600 and 1800, but it appears to be very minor differences of the surge margins at the speedlind 1000 and 1300 where the pressure ratio is lower than 3. These findings are consistent with the common understanding of the ported shroud function, which is to enhance compressor surge margins when the pressure ratio is above 3 and increase choke margins at very high speed.

In Fig. 8.4, the predicted and measured compressor efficiency is compared. One can see that the CFD calculations over-estimates the efficiency, which is the 


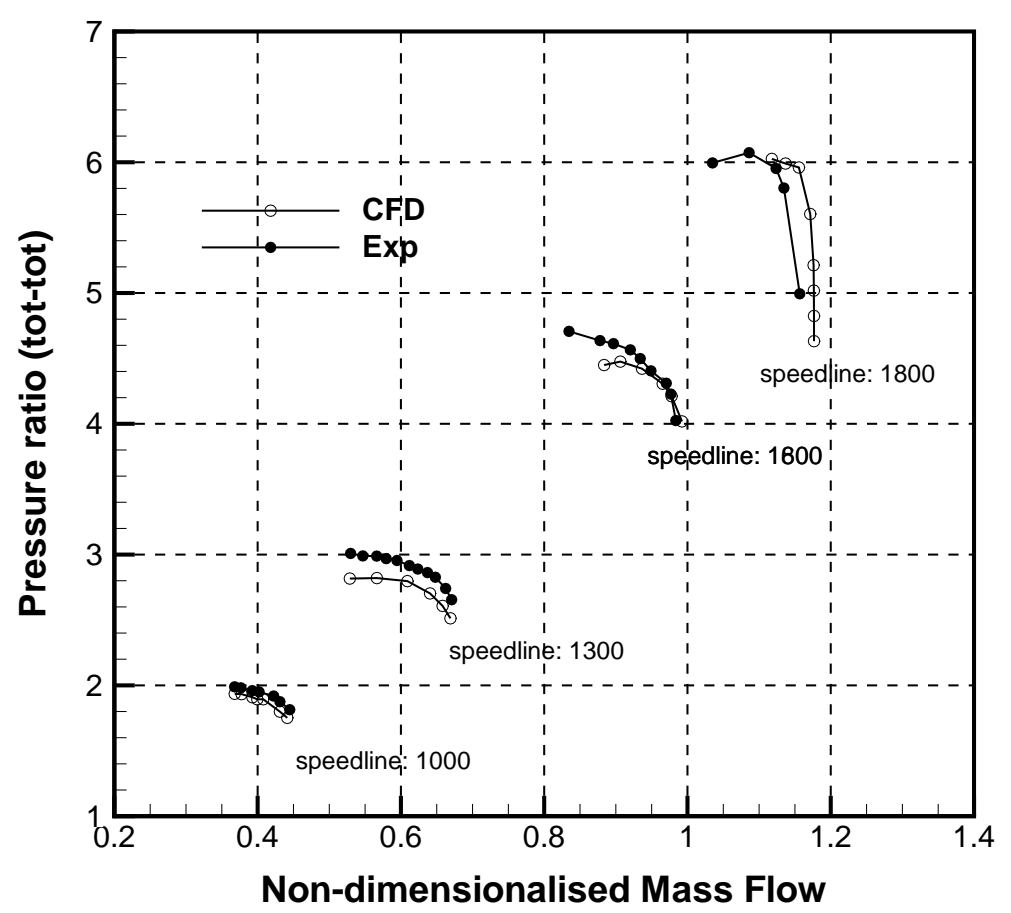

Figure 8.3: Comparisons between the predicted and measured redesigned compressor's characteristics: pressure ratio

same case shown in Fig. 7.7 for the baseline design. Therefore, the CFD set up for two different compressor stages leads to consistent predictions when compared against the test data.

\subsection{Measurements of redesigned compressor stage maps}

In Figs. 8.5 and 8.6 the measured compressor characteristics of the two designs are compared at various speedlines. As expected from CFD predictions, the redesigned compressor produces lower pressure ratio and efficiency at lower speeds. After the speed is increased to about 1700, it starts to show the advantages of the pressure ratio and efficiency, which agrees well with the original design requirement to achieve better performance at higher speeds. The internal flow field analysis will be presented later to explain the mechanisms why the redesigned 


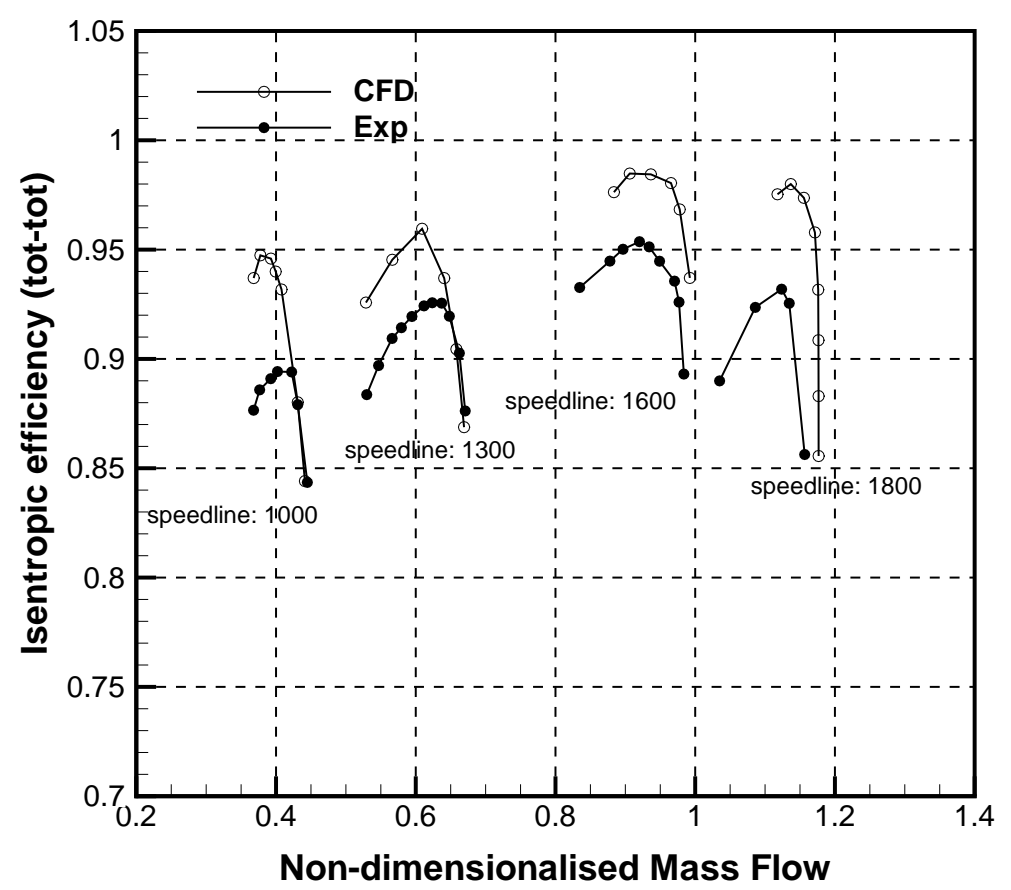

Figure 8.4: Comparisons between the predicted and measured redesigned compressor's characteristics: efficiency

impeller behaves better at higher speeds.

A better understanding between these two compressors' performance can be obtained from the contour plots presented in Figs. 8.7a and 8.7b. It can be seen that the baseline compressor has its high efficiency zone at medium rpms whereas the redesigned compressor preserves its high efficiency zone at higher turbocharger speeds. In addition, it is clear that the choke line of the two compressors are almost identical except that the redesigned compressor has a slightly smaller choke margin at the highest speed. On the other hand it is easy to see from Fig. $8.7 \mathrm{c}$ that a gain of the surge margin is acquired after the speedline 1600 , resulting in a bigger operating envelope comparing with the baseline one.

\subsection{Measured acoustic noise}

In this section we will present the acoustic measurements of two compressor stages. It will be shown that the redesigned impeller causes significantly less 


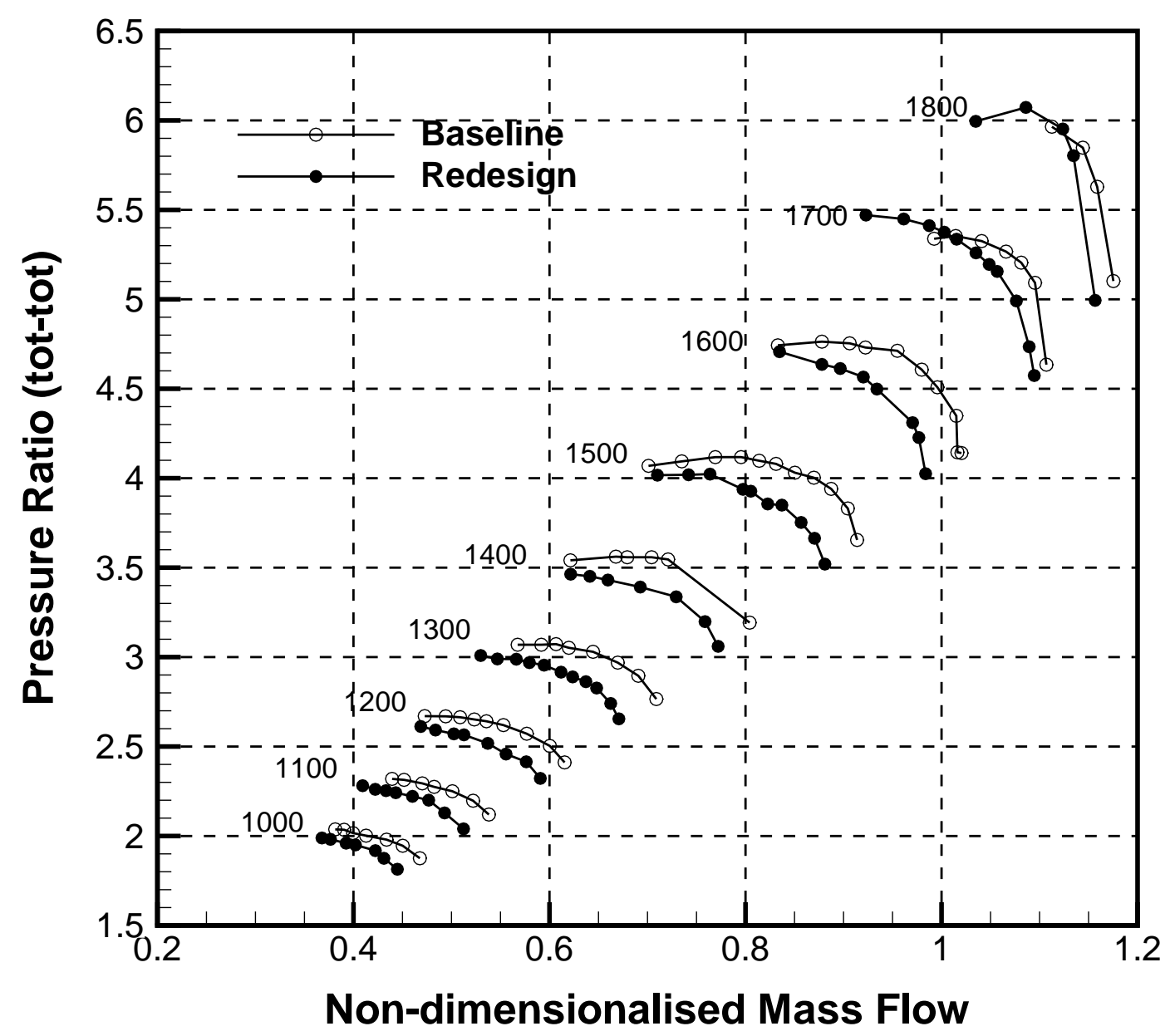

Figure 8.5: Comparisons of the measured stage pressure ratios of baseline and redesign compressors at different operating speedlines 


\section{EVALUATIONS OF THE INVERSELY DESIGNED IMPELLER}

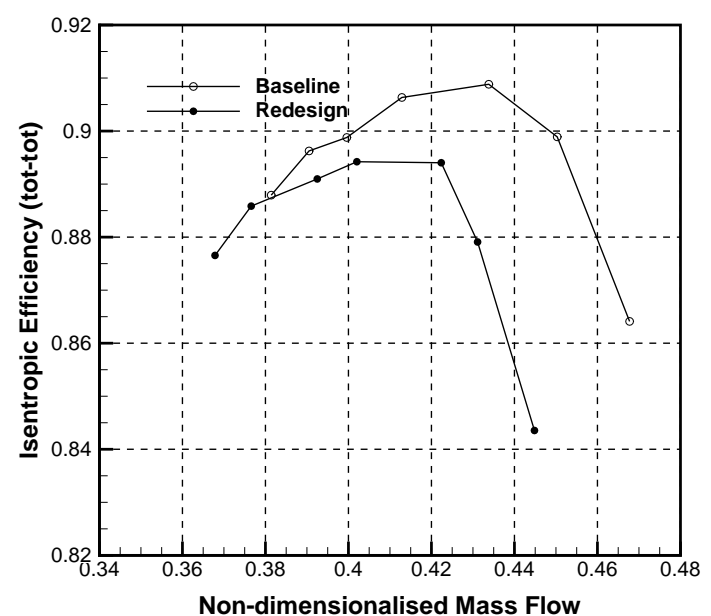

(a) speedline 1000

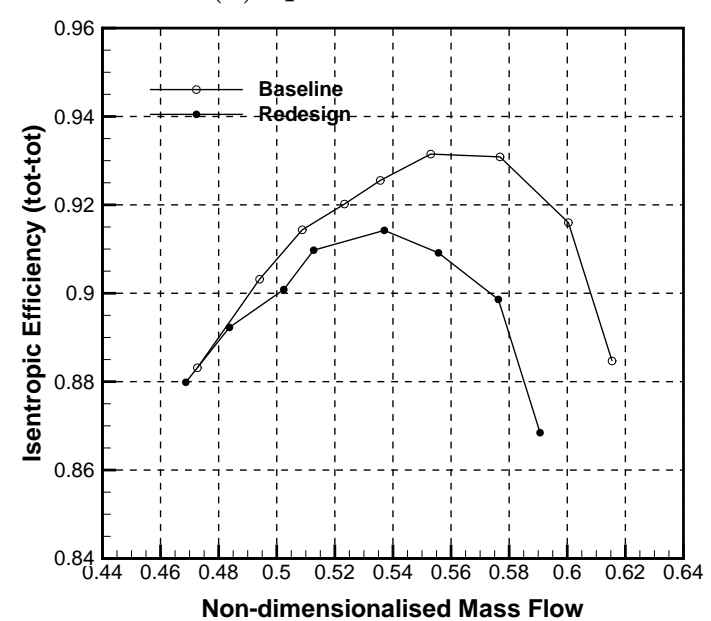

(c) speedline 1200

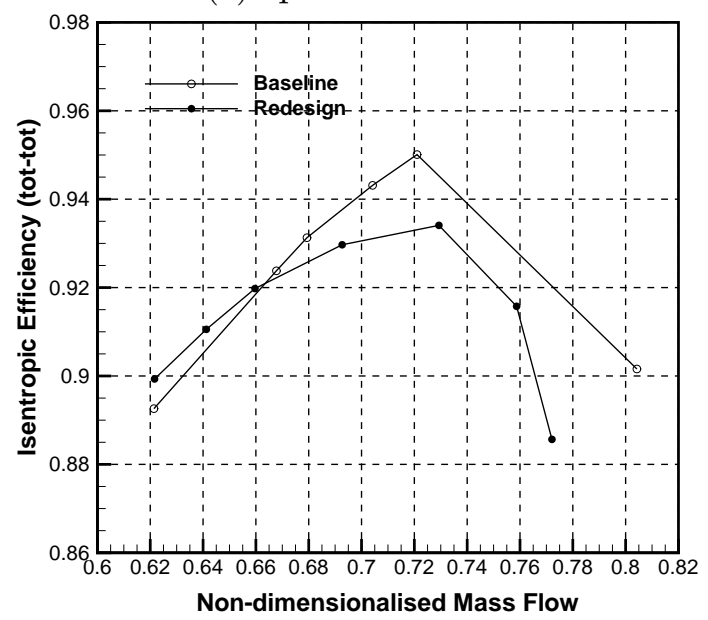

(e) speedline 1400

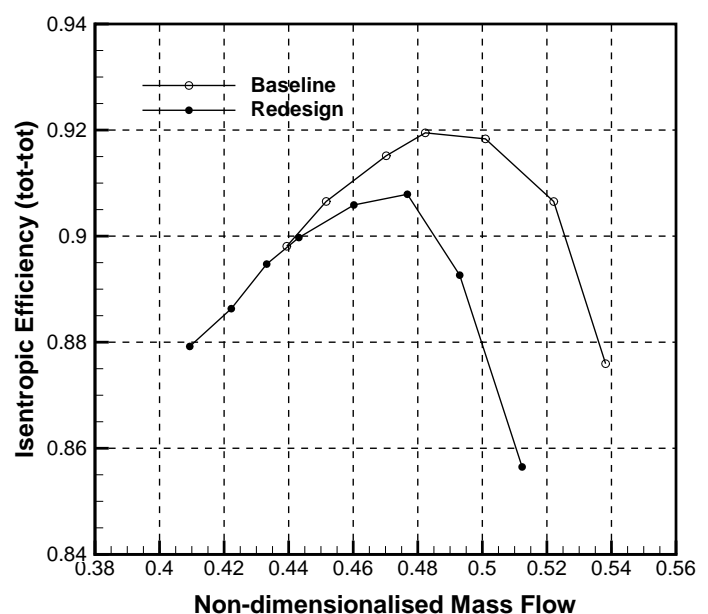

(b) speedline 1100

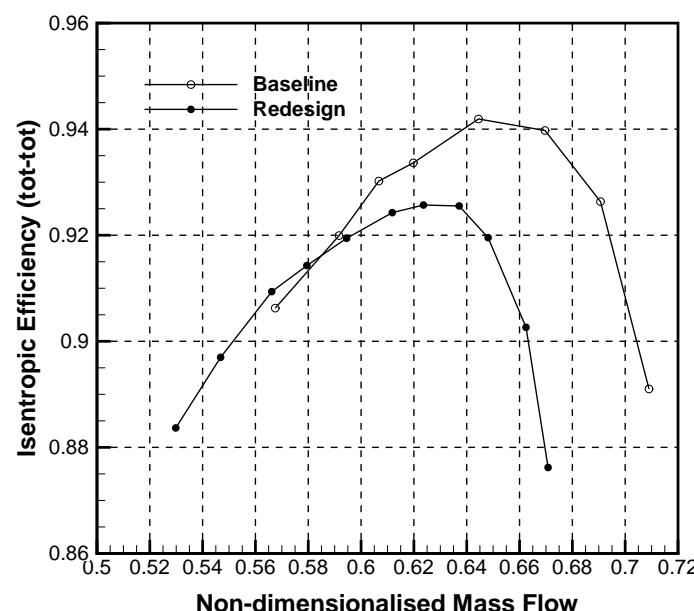

(d) speedline 1300

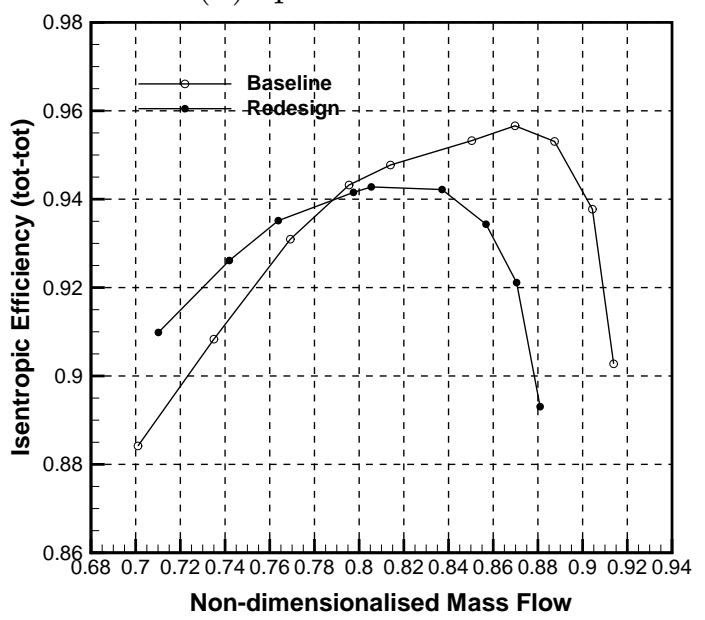

(f) speedline 1500

Figure 8.6: Comparisons of the measured stage efficiency (non-dimensionalised) of baseline and redesign compressors at different operating speedlines 


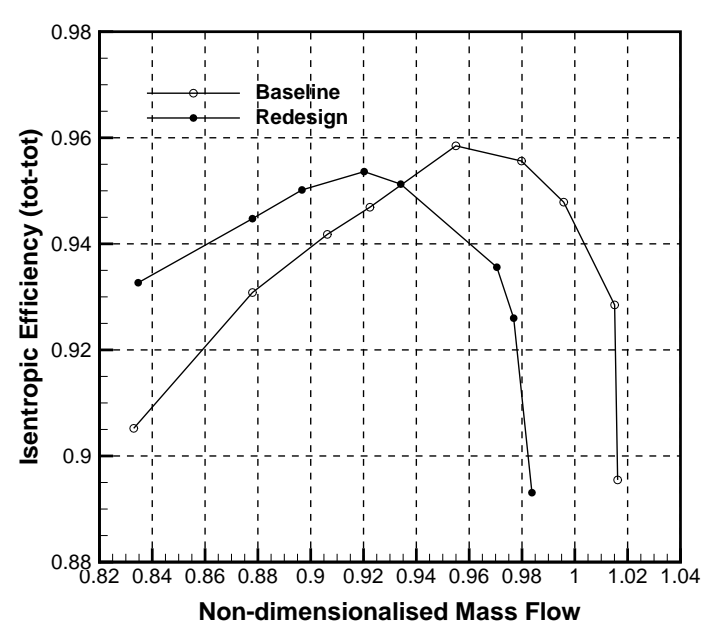

(g) speedline 1600

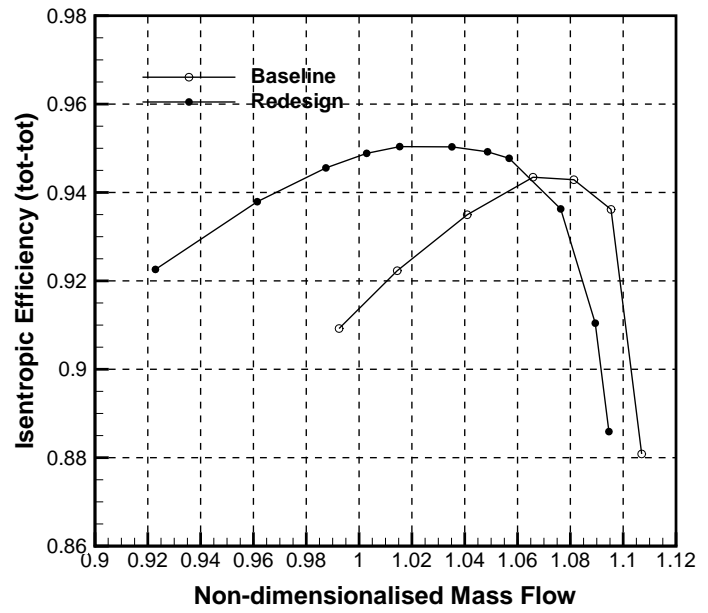

(h) speedline 1700

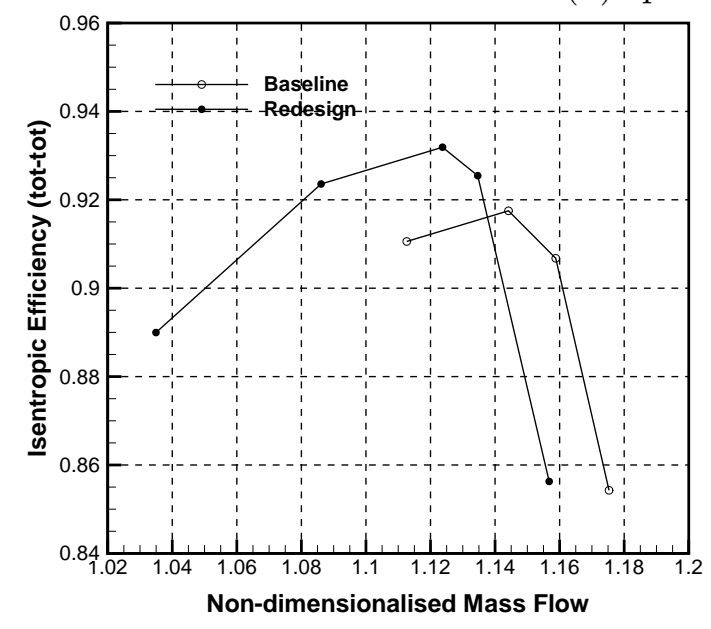

(i) speedline 1800

Figure 8.6: (continued) Comparisons of the measured stage efficiency (nondimensionalised) of baseline and redesign compressors at different operating speedlines 


\section{EVALUATIONS OF THE INVERSELY DESIGNED IMPELLER}

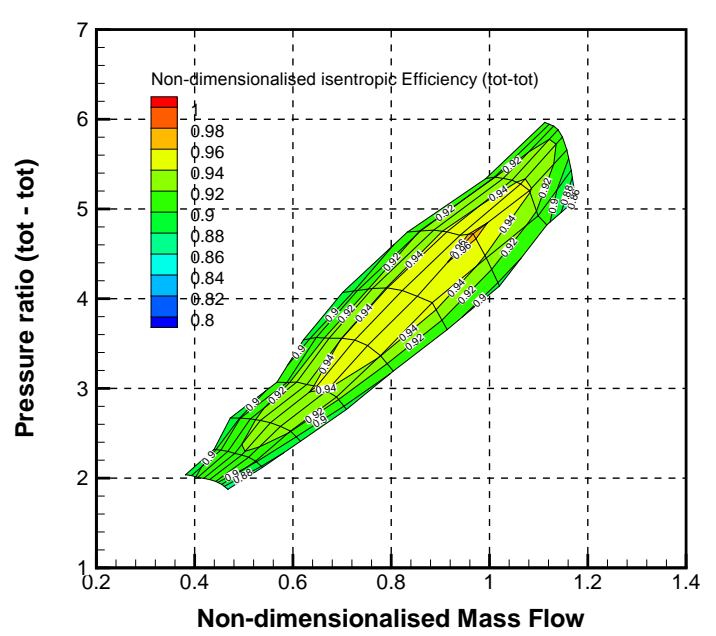

(a) Baseline compressor performance contour

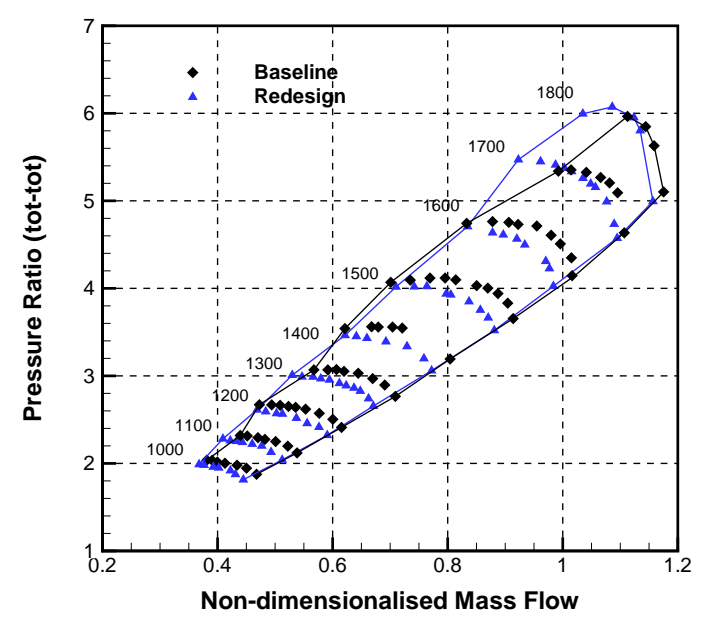

(c) Comparisons of the two designs' working envelope

Figure 8.7: Comparisons of the two compressors' performance and their operating ranges. (Experimental measurements) 


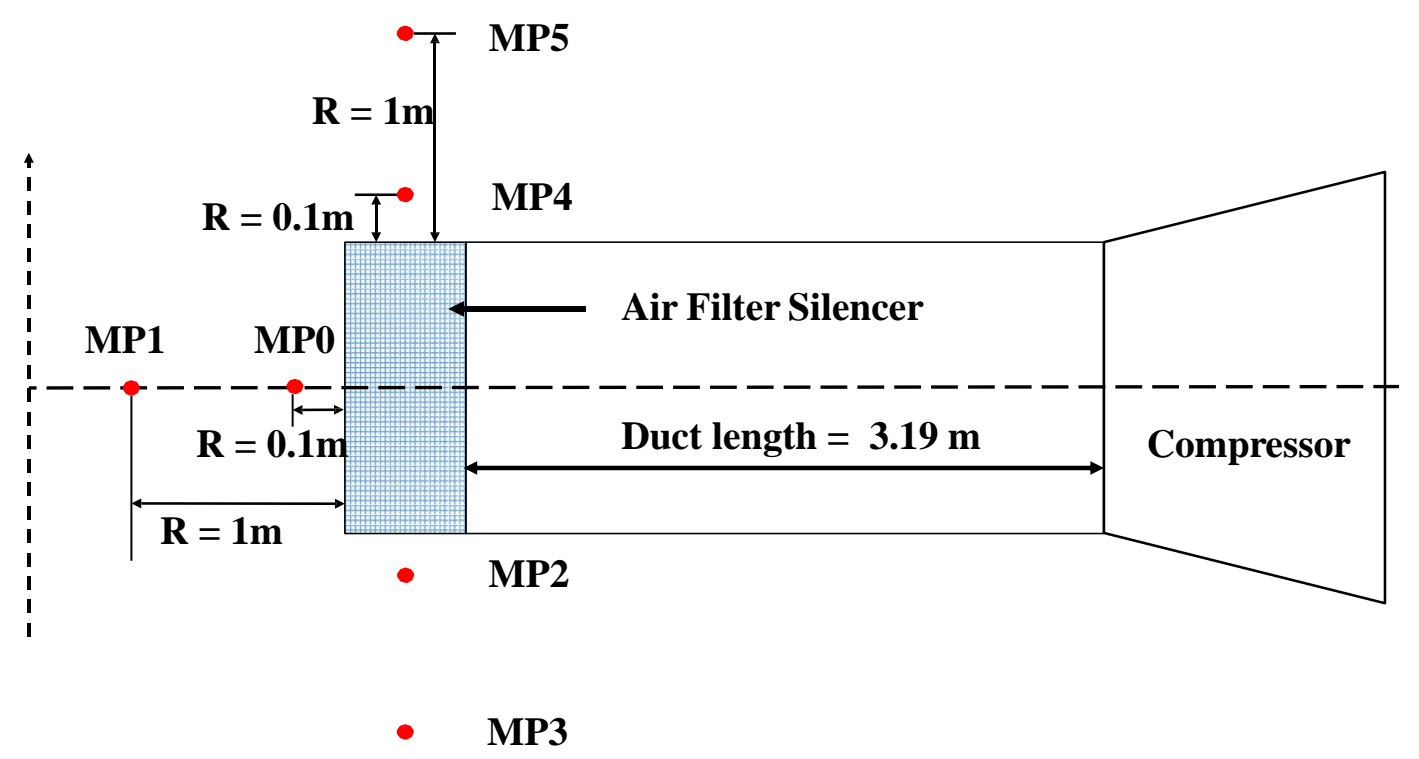

Figure 8.8: Different noise measurement positions. (MP2 and 3 are symmetric to MP4 and 5)

noise than the baseline one.

The acoustic measurements were conducted by Kay \& Heyes [2014] on the test stand at Napier Turbochargers Ltd. A simplified schematic of the test stand is presented in the Fig. 8.8, which shows the six different measurement positions surrounding the AFS (air filter silencer) placed upstream of the compressor inlet. At each MP the sound level was measured three times and the average values were taken as the effective results. Bruel and Kjaer 2260 Sound Investigator, Serial no 2350032, was used to record the sound levels. The analyser was set up to take the average sound levels in $1 / 3$ spectrum format over a 10 second period.

Figure 8.9 compares the measured sound levels of two impellers at six MPs with the turbo speeds from 1000 to 1800 . It can be seen that both impeller's sound level increases as the turbo speed increases, and in general, the redesigned impeller gives rise to lower sound levels at every MP, and it has more advantages of lower noise levels at higher speeds.

In Figs 8.11 to 8.16 are shown the noise spectral characteristics at different measurement locations and speeds. It is easy to see that the redesigned compressor has a significantly lower tone levels at the blade passing frequency and its harmonics, and this advantage would be amplified as the speed increases. 


\section{EVALUATIONS OF THE INVERSELY DESIGNED IMPELLER}

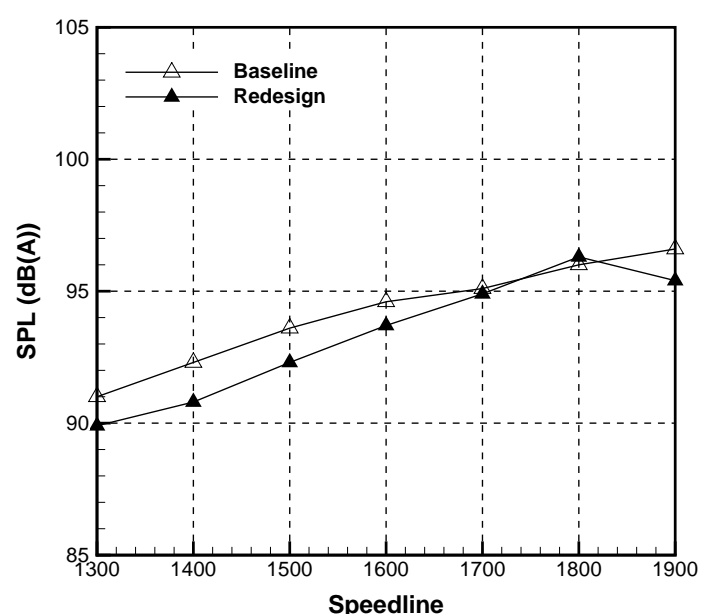

(a) SPL at MP0

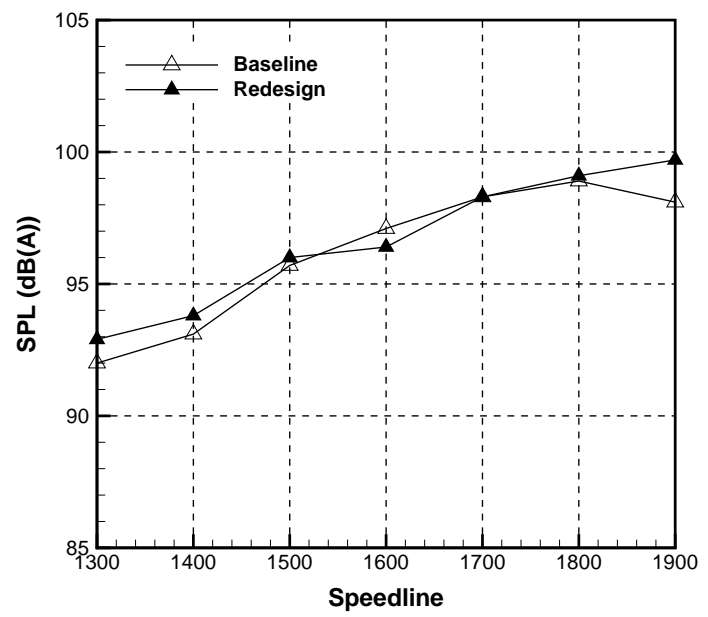

(c) SPL at MP2

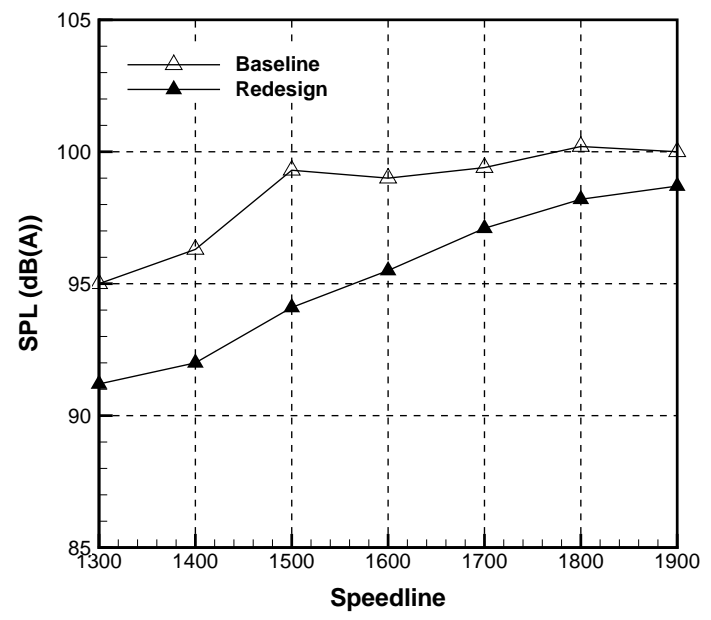

(e) SPL at MP4

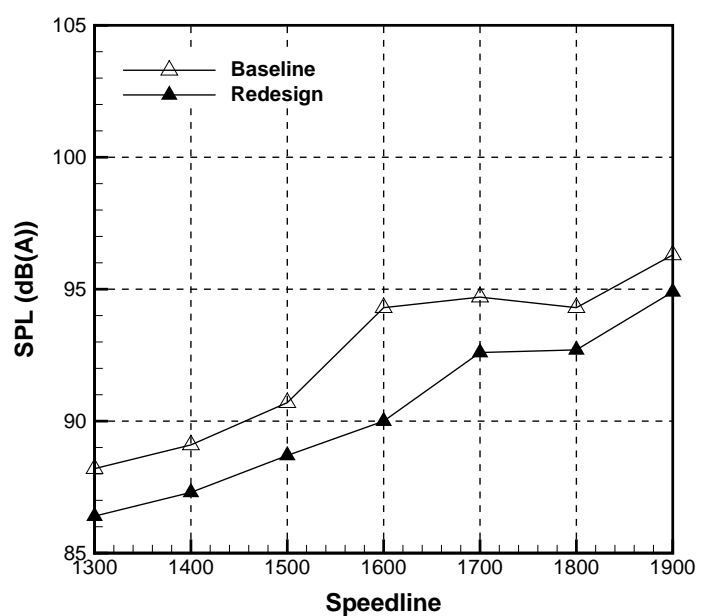

(b) SPL at MP1

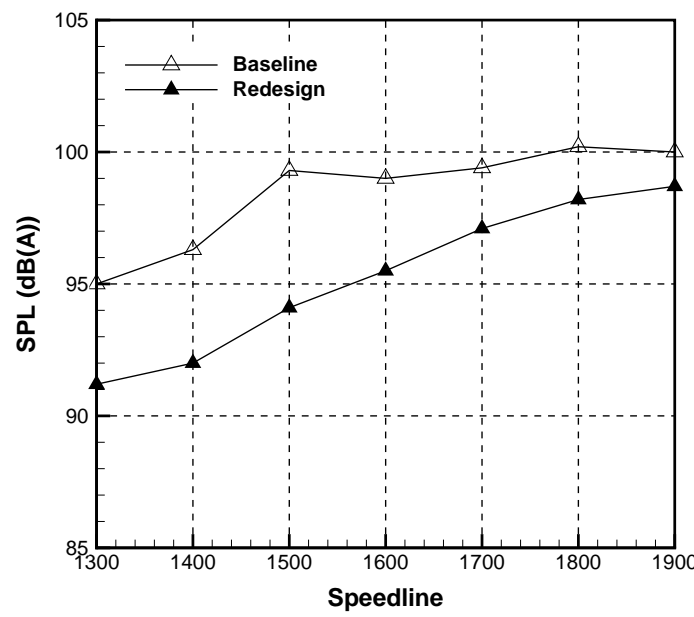

(d) SPL at MP3

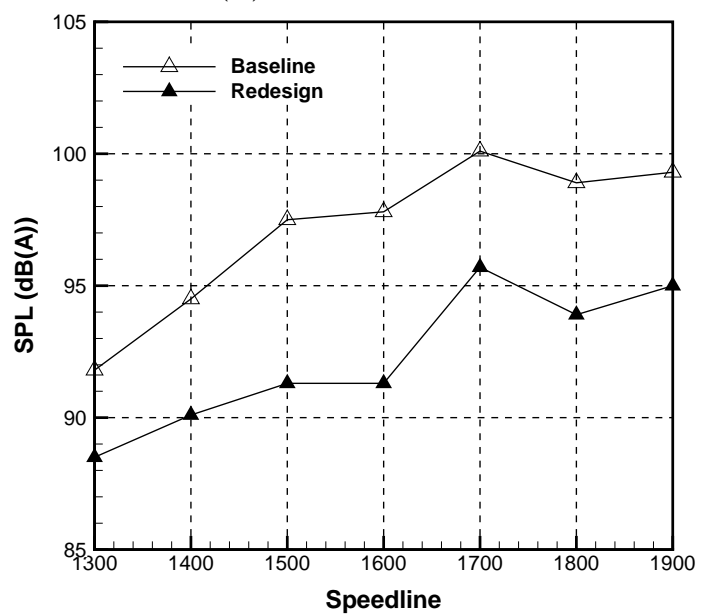

(f) SPL at MP5

Figure 8.9: Sound level measured at the inlet for different positions 


\section{EVALUATIONS OF THE INVERSELY DESIGNED IMPELLER}

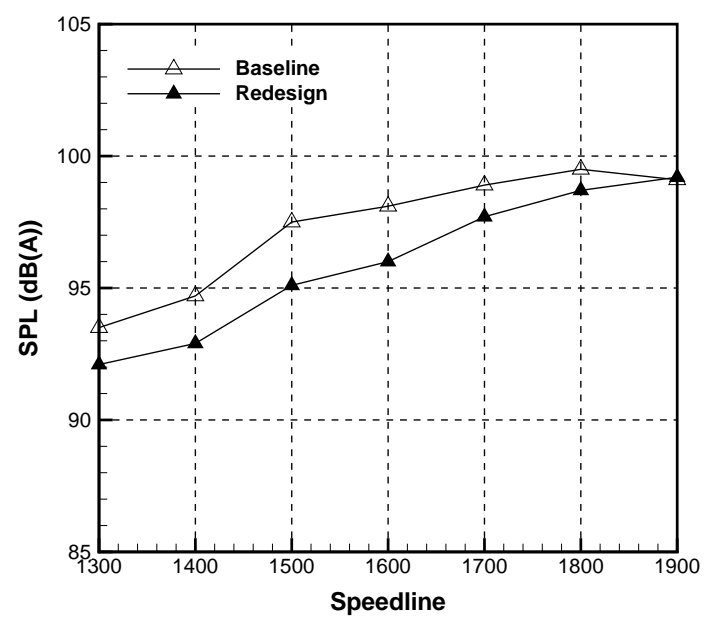

(a) Average SPL at MP 2 and 4

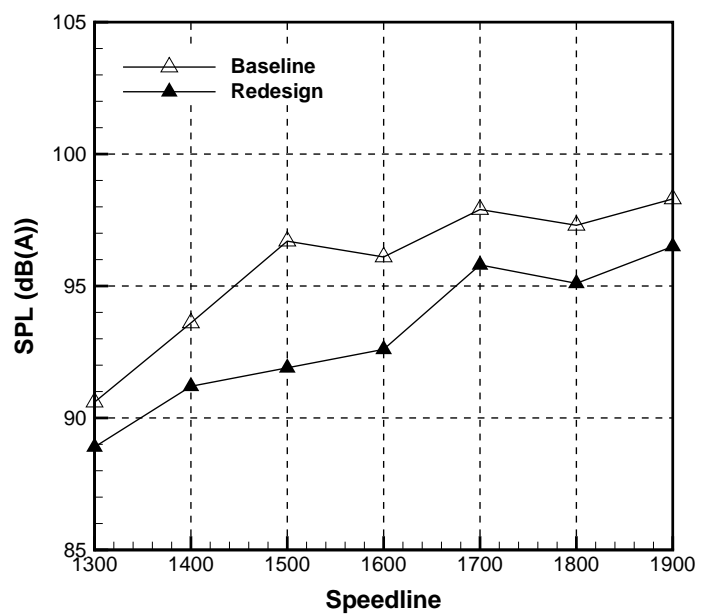

(b) SAverage SPL at MP 3 and 5

Figure 8.10: Average sound levels measured at position 2, 4 and position 3, 5
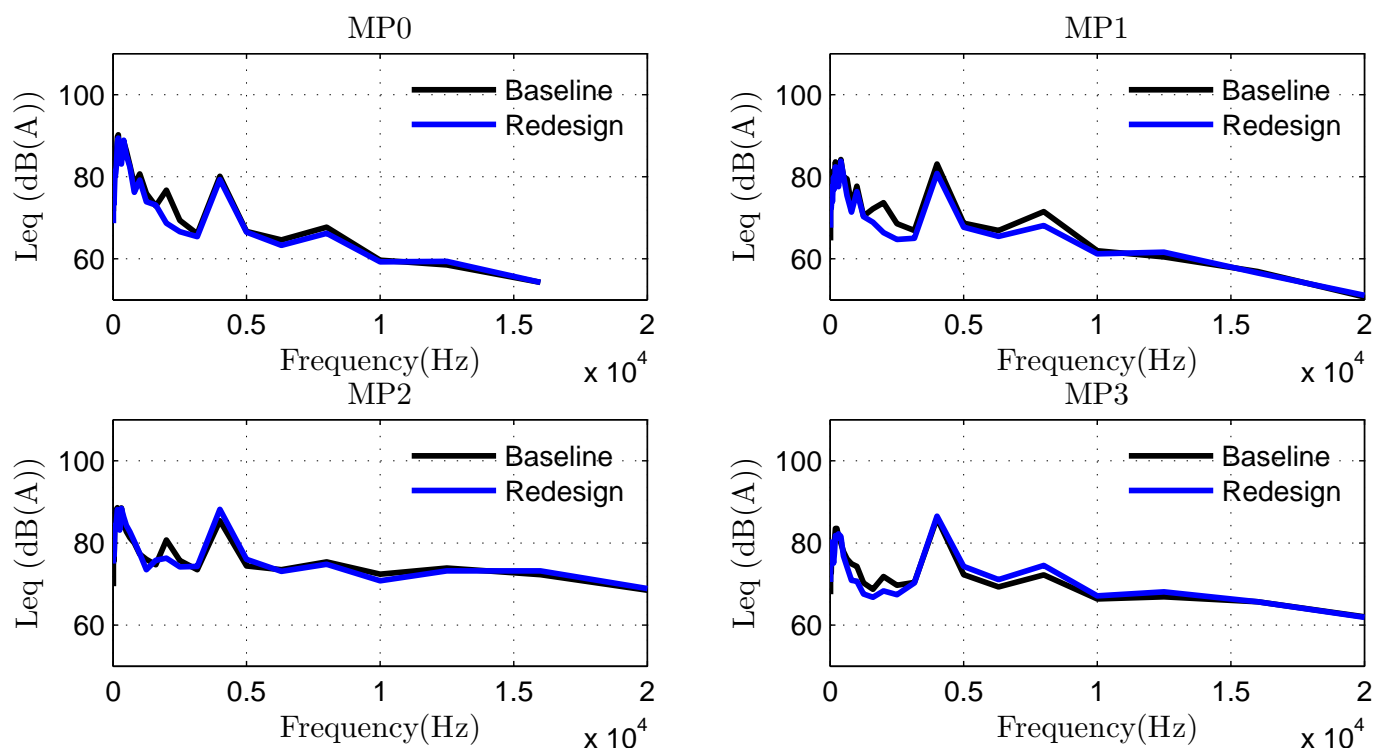

MP3

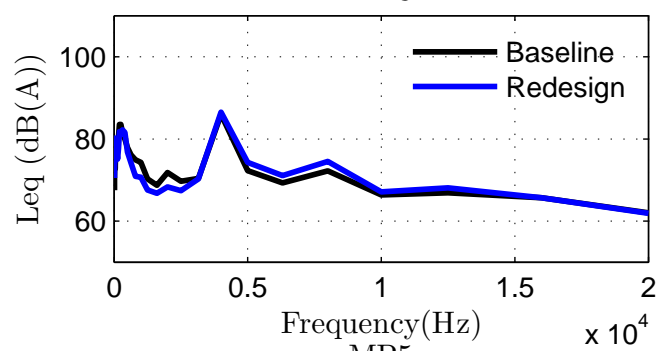

MP4

MP5
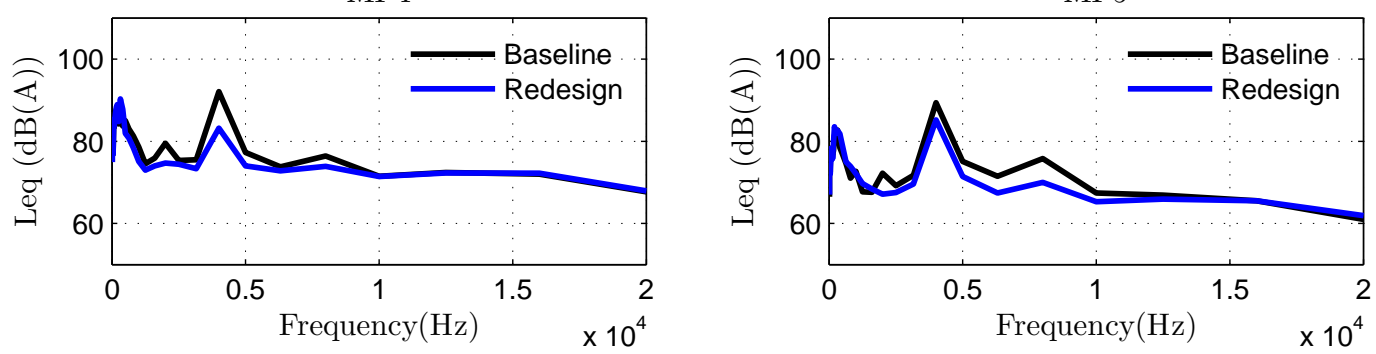

Figure 8.11: Comparisons of the measured spectral characteristics of baseline and redesign impellers at speedline 1300 

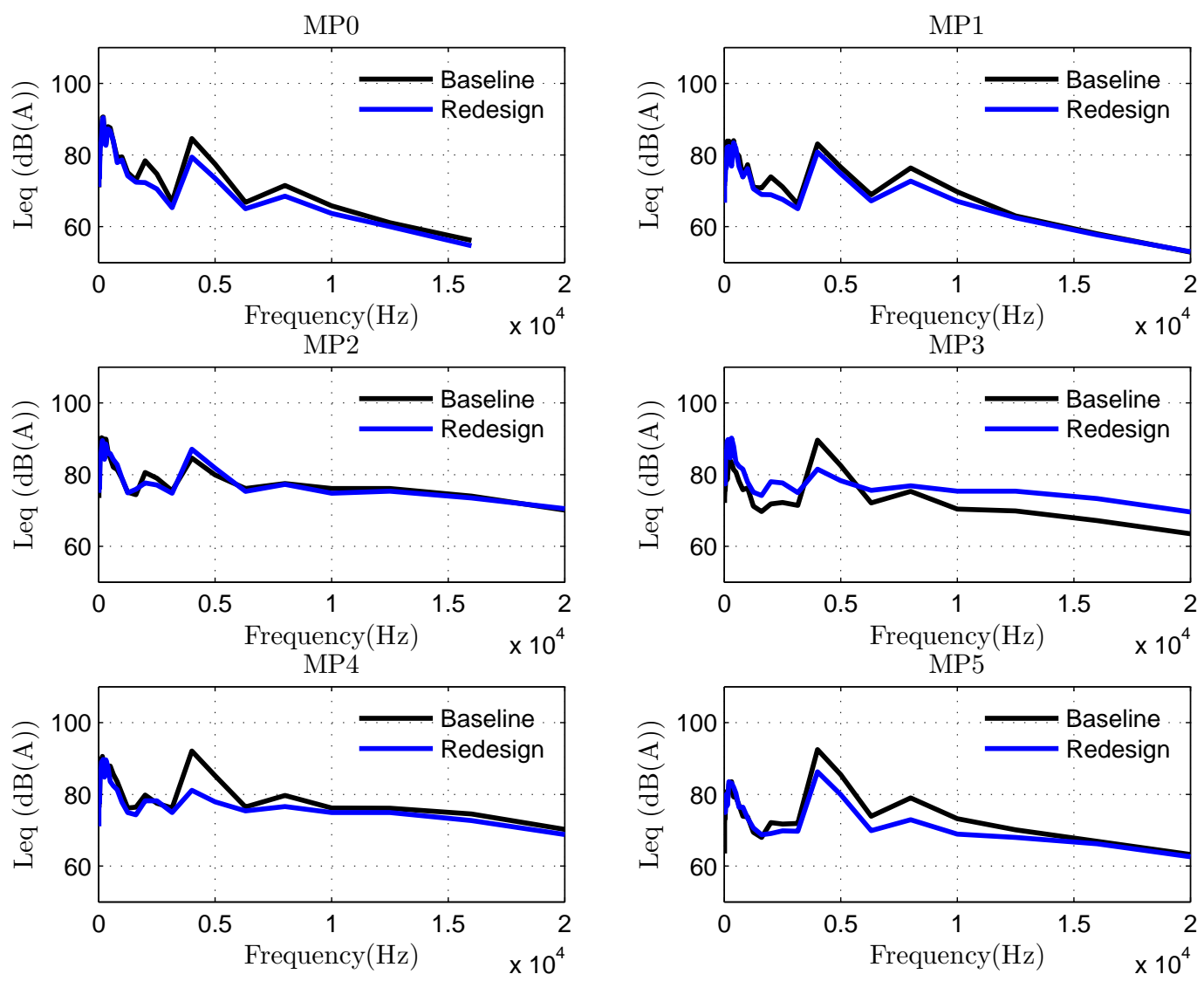

Figure 8.12: Comparisons of the measured spectral characteristics of baseline and redesign impellers at speedline 1400 

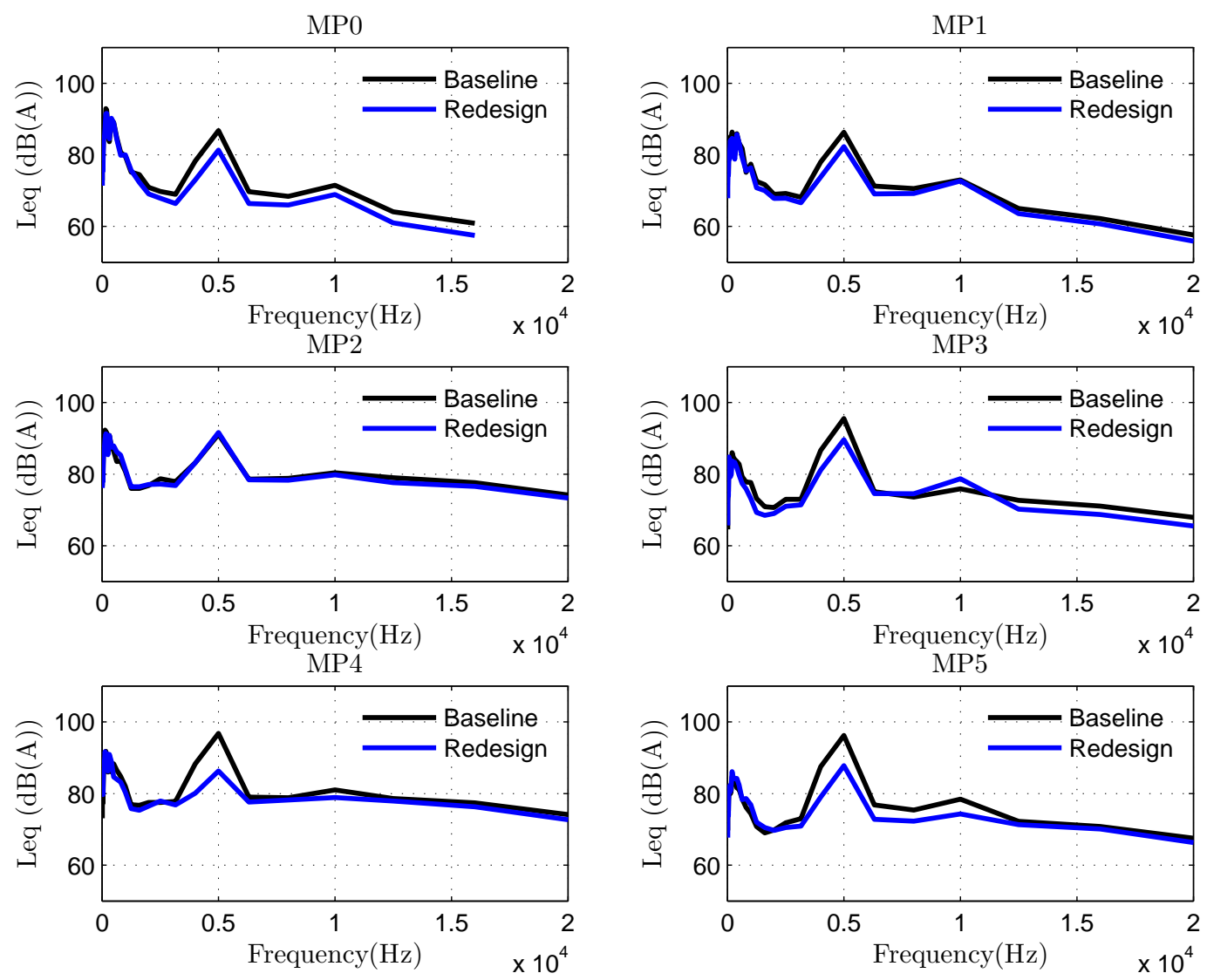

Figure 8.13: Comparisons of the measured spectral characteristics of baseline and redesign impellers at speedline 1500 

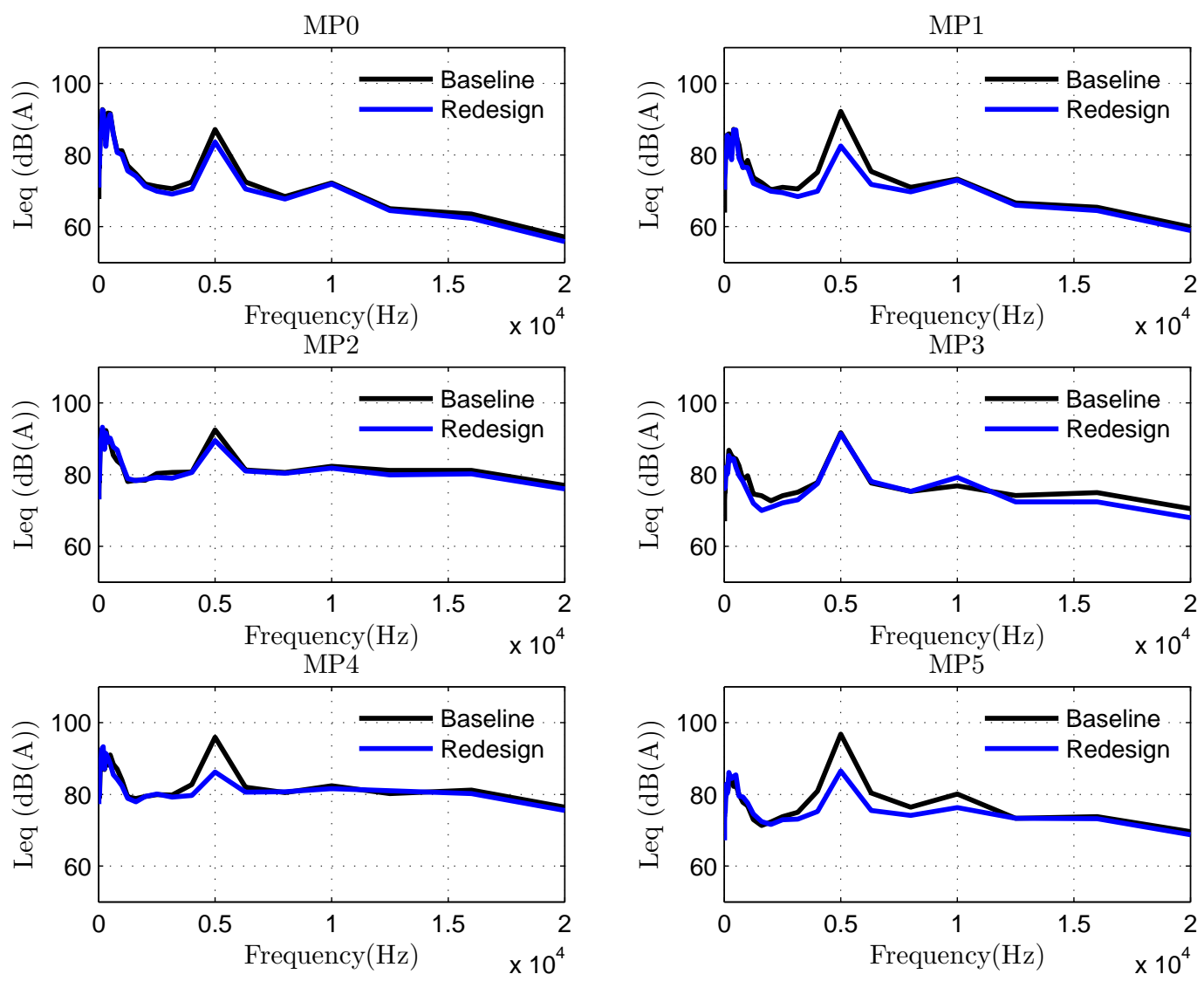

Figure 8.14: Comparisons of the measured spectral characteristics of baseline and redesign impellers at speedline 1600 

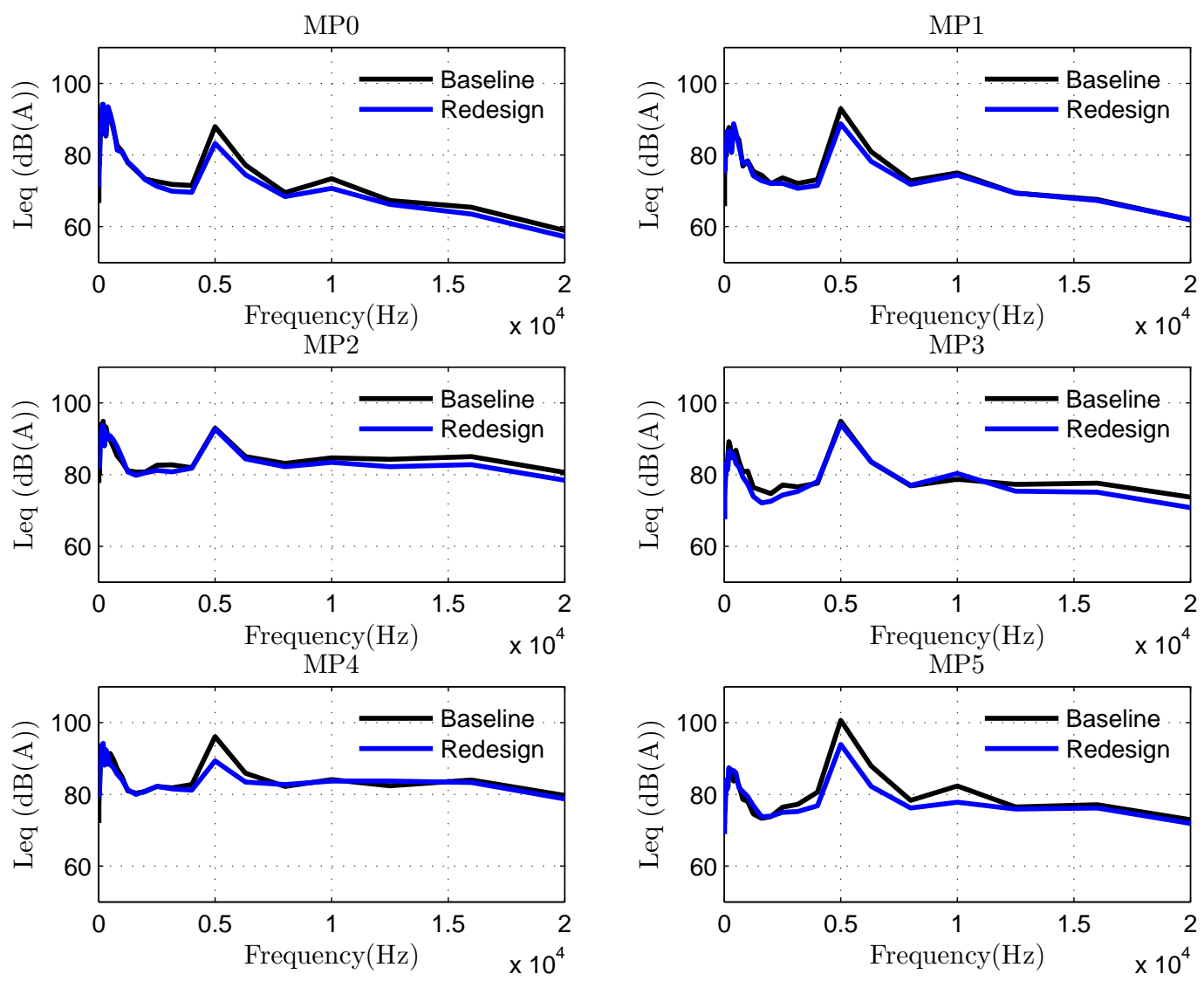

Figure 8.15: Comparisons of the measured spectral characteristics of baseline and redesign impellers at speedline 1700 

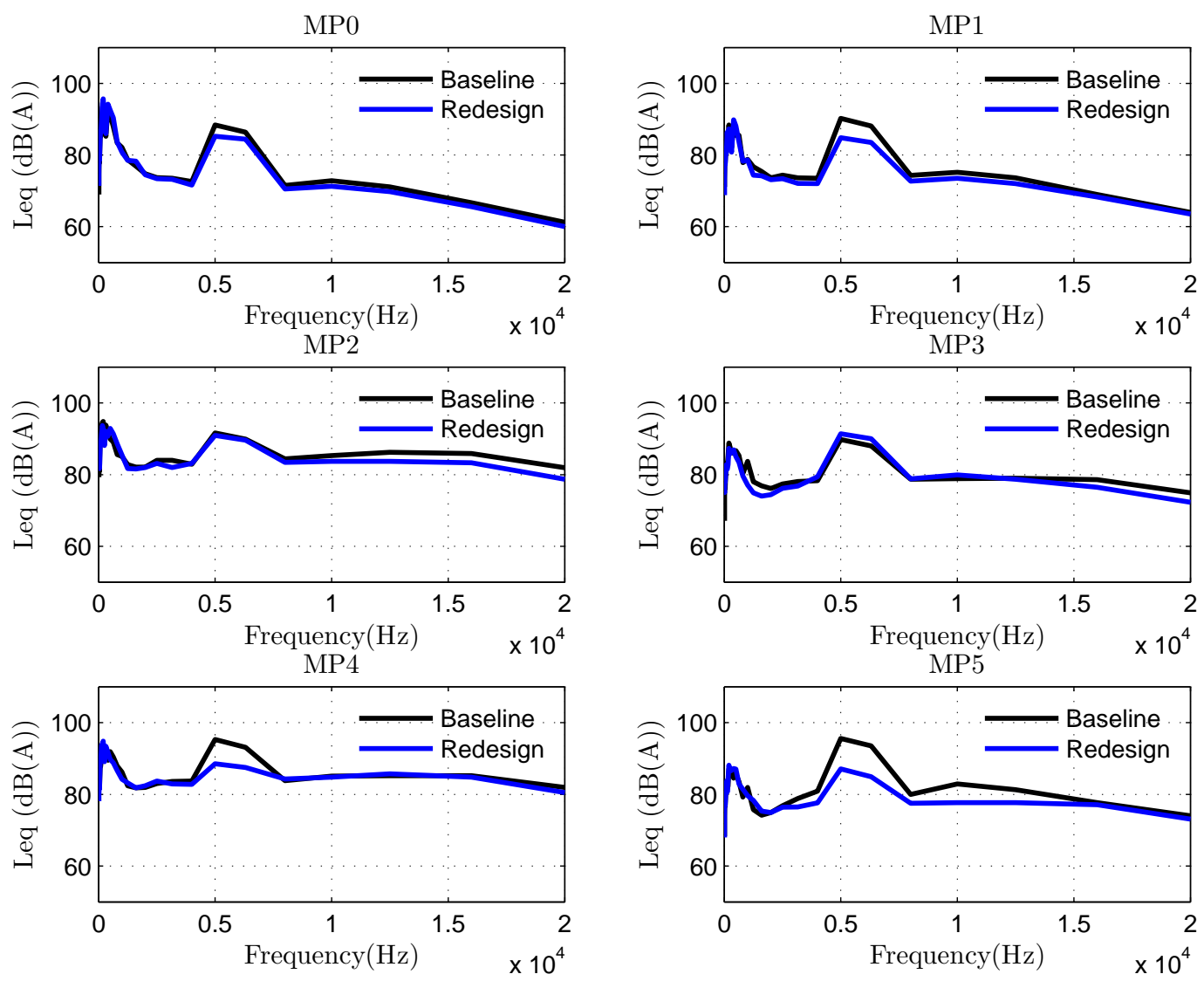

Figure 8.16: Comparisons of the measured spectral characteristics of baseline and redesign impellers at speedline 1800 

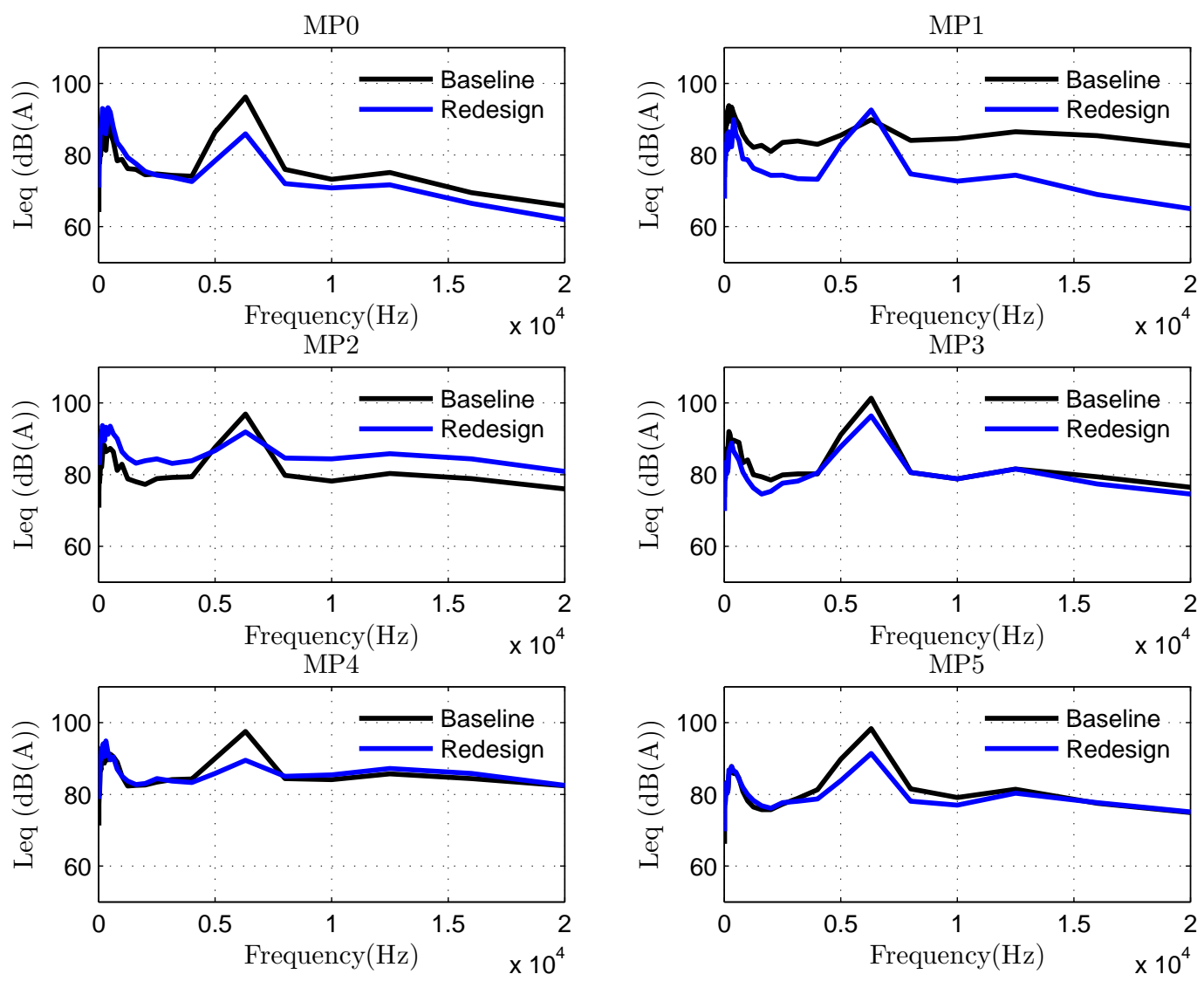

Figure 8.17: Comparisons of the measured spectral characteristics of baseline and redesign impellers at speedline 1900 


\section{EVALUATIONS OF THE INVERSELY DESIGNED IMPELLER}

\subsection{Comparisons of flow physics}

Having demonstrated above that the redesigned impeller leads to better aerodynamic and aeroacoustic performance above, flow physics in the baseline and redesigned blade channels are compared in this section. We will focus on the flow structures at choke, stall, and peak efficiency conditions. The major losses in a transonic centrifugal compressor such as shocks, flow separation, secondary flow, tip leakage, and exit flow uniformity will be investigated for both designs.

\subsubsection{Choke margins}

As has been mentioned that the throat area is normally regarded as a very important parameter to monitor during the design process since it determines compressor's choking condition. It was decided at the beginning that the redesigned impeller must have similar-scale throat area to the baseline so as to ensure the similar choke margins. However the test data has shown that the redesigned compressor has noticeable smaller choke margins at almost every speedline, which is a contradiction to conventional design theory. To understand how this unusual phenomenon can happen, we need to investigate the details of the flow field in the blade channel.

In Figs 8.18 - 8.26 are presented the relative Mach number distributions at the different spanwise positions from hub to shroud. It should be noticed that choking at the lower rpms occur in a region close to the impeller trailing edge, but the conventional throat area is usually assumed in the impeller leading edge, as is shown in Fig. 8.27. It represents the area of a plane that is in the vicinity of the inducer and constructed by the shortest lines connecting the pressure and suction side at different spanwise locations. As choking does not happen in this area, the throat area cannot be used to control the choke margin. Thus, we need to seek another explanation why the redesigned impeller has smaller choke margins, particularly at lower rpms.

If we re-think about the flow velocity changes in the compressor channel, we can see that the flow is accelerated in the channel due to its contracting shape. As the incoming flow rate increases for a compressor operating at lower rpms, the flow can attain Mach number 1 near the trailing edge first, and hence chokes. 


\section{EVALUATIONS OF THE INVERSELY DESIGNED IMPELLER}
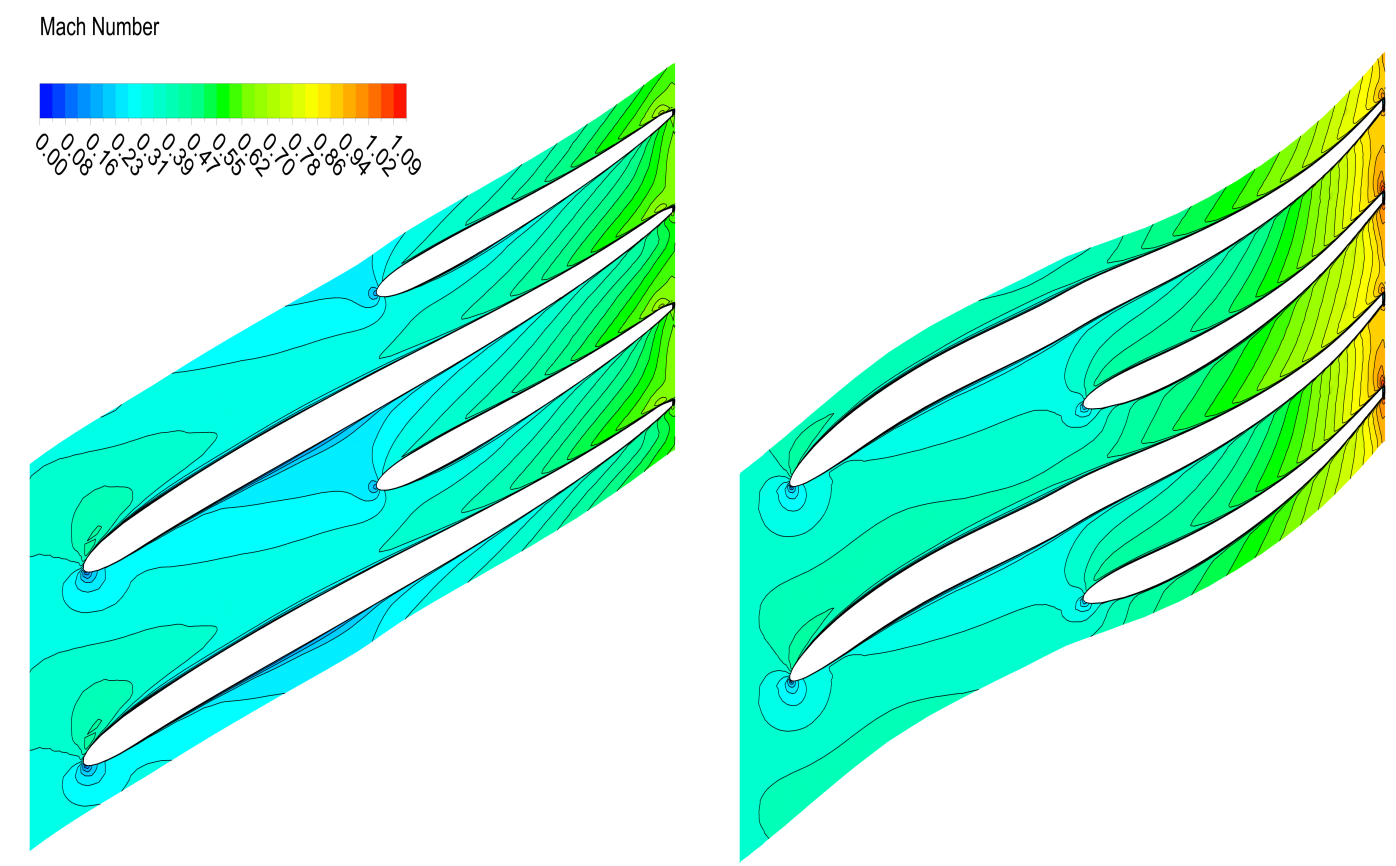

Figure 8.18: Comparisons of the relative Mach number distribution between the baseline (left) and redesign (right) impeller near the hub at speedline 1000

Although the meridional housing of the baseline and redesign is the same, the redesigned impeller channel has smaller effective area for the flow to pass due to its increased thickness at the blade root, which can be seen in Figs. 7.11 and 7.12. Therefore, the redesigned impeller chokes earlier when operating at lower rpms. As the rpm increases, the flow would reach Mach number 1 in the inducer first before getting to the exduer region. Thus, with similar throat area of the two design, similar choke margin shall be obtained at high rpms, as shown in Fig. 8.5 .

\subsubsection{Tip leakage flow}

The tip leakage flow is one of the most important loss sources in centrifugal machines. Reduced tip leakage flow would benefit the aerodynamic performance, such as efficiency and surge margin, and help to reduce the broadband noise. Figure 8.31 shows the 3D streamlines behaviours for both baseline and redesigned impeller channels. There is noticeably more flow crossing the tip gap from the 


\section{EVALUATIONS OF THE INVERSELY DESIGNED IMPELLER}
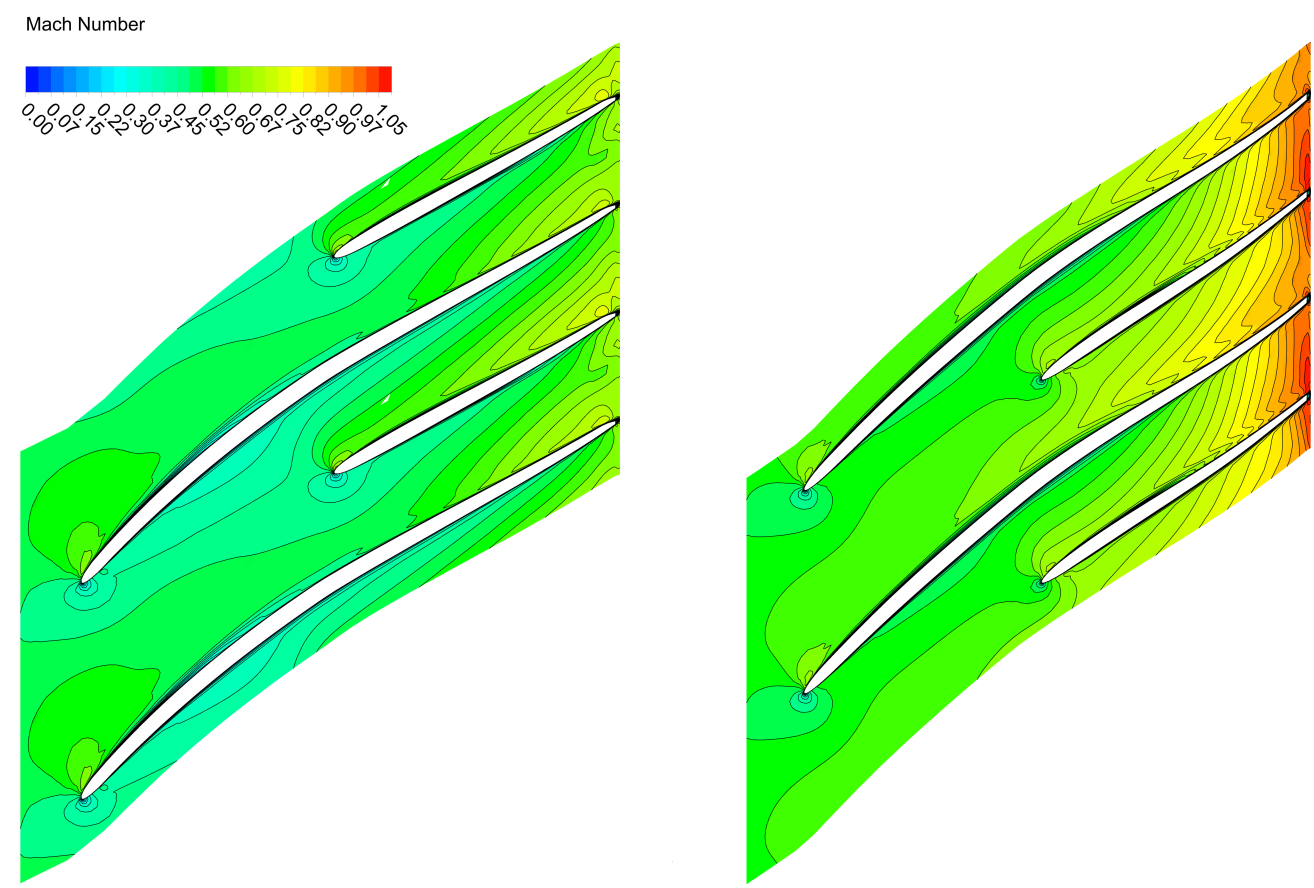

Figure 8.19: Comparisons of the relative Mach number distribution between the baseline (left) and redesign (right) impeller at the midspan at speedline 1000

pressure side to suction side in the baseline channel, whose tip leakage flow exhibits a stronger vortical nature. A better view of tip leakage flow in the baseline and redesigned impeller can be seen in Fig. 8.40, which shows significant more leakage flow coming from the suction side of the baseline full blade than the redesign.

\subsubsection{Flow separations}

The flow separations in the blade channel are also a very important loss mechanism of turbomachinery efficiency and broadband noise source. In Figs 8.32 and 8.33 are shown the surface stream line and relative Mach number distribution on the full and splitter blades' suction surfaces. One can easily see that the redesigned splitter has a much better streamline structure attached on the blade surface and the low momentum zone is considerably smaller.

In Figs. 8.34 and 8.35 are presented the velocity vectors on the suction surface of the baseline and redesigned full and splitter blades respectively. On the suction 


\section{EVALUATIONS OF THE INVERSELY DESIGNED IMPELLER}
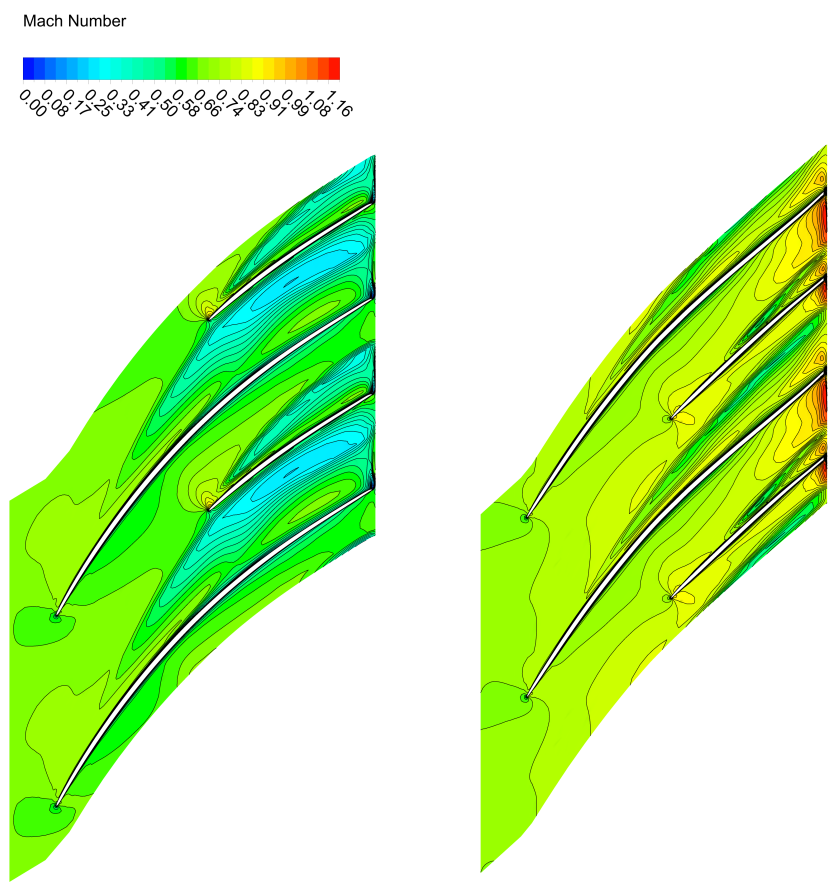

Figure 8.20: Comparisons of the relative Mach number distribution between the baseline (left) and redesign (right) impeller near the shroud at speedline 1000

surface of the baseline full blade, it is easy to notice flow moving from the suction to pressure side through the tip gap around the mid-chord of the blade. A similar but less severe phenomenon can also be seen on the redesigned full blade's suction side. If one takes a closer scrutiny of the trailing edge shown in Fig. 8.34, it can be seen that the flow on the hub gradually moves towards a higher spanwise position on the baseline design, which is not observable on the redesigned full blade trailing edge. The flow in the region near the trailing edge of the redesigned full blade moves perfectly along the streamwise direction.

The flow velocities on the suction side of the splitter blade are quite distinct. A clear separation of the flow can be seen on the baseline splitter, and there are clear tendencies that the flow starts to escape from the suction side and slide to the pressure side in the frontal area of the splitter blade. The flow on the redesigned splitter blade fully attaches to its surface and smoothly moves along the streamwise direction from the leading edge to trailing edge. 


\section{EVALUATIONS OF THE INVERSELY DESIGNED IMPELLER}
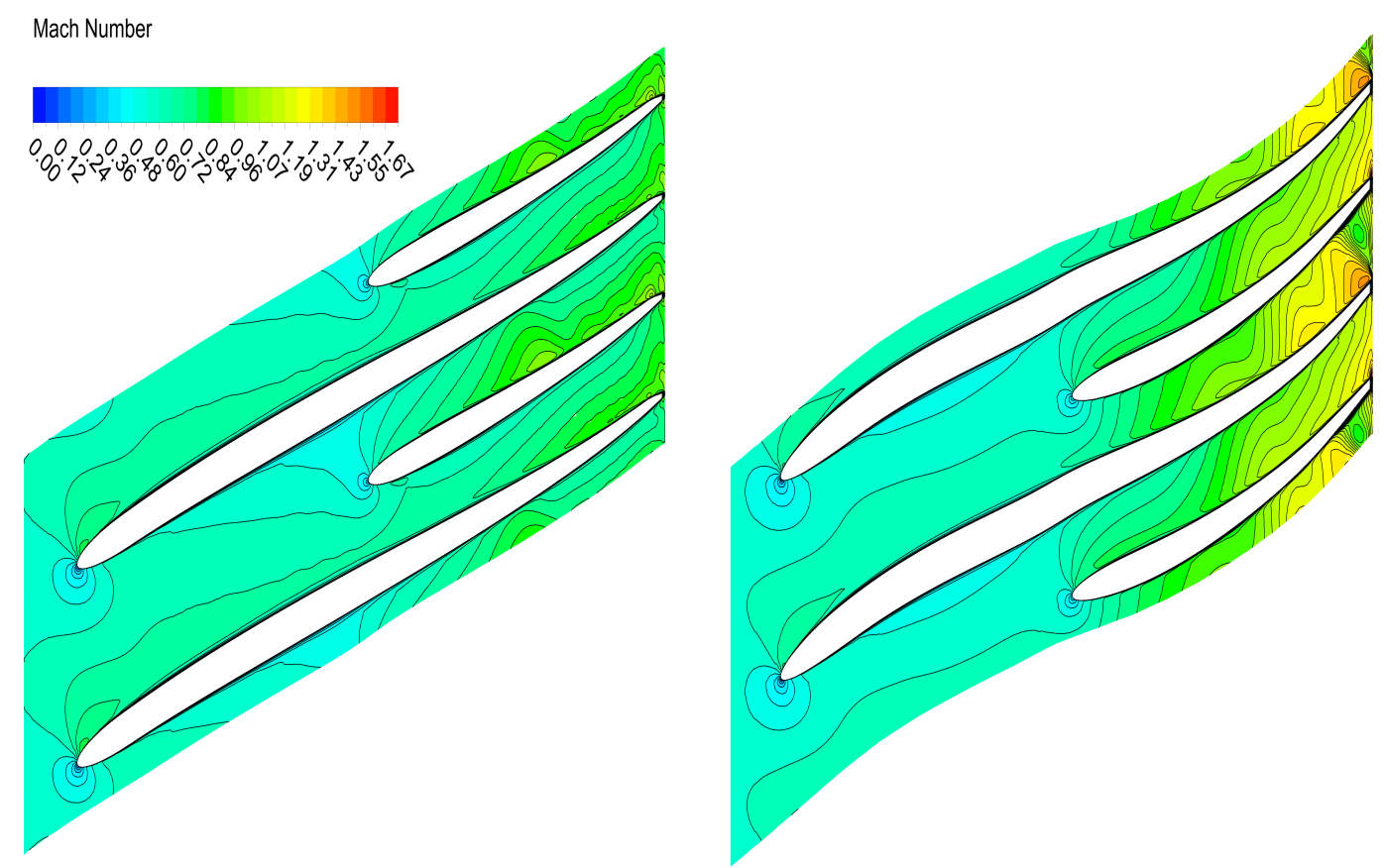

Figure 8.21: Comparisons of the relative Mach number distribution between the baseline (left) and redesign (right) impeller near the hub at speedline 1500

\subsubsection{Exit flow uniformity}

The exit flow uniformity plays a rather important role for determining the overall stage performance for the compressor unit. After leaving the rotating blades it will interact with the vaned diffuser, where flow with strong non-uniformity will generate large losses. Figure 8.36 illustrates the Mach number distribution in a plane offset from the impeller trailing edge by $1 \mathrm{~mm}$. Clearly, the exit flow structures between the baseline and redesign are quite distinct. On the exit of baseline impeller there is a clear jet-wake pattern flow which consists of a portion of flow with very low velocity. Such big velocity variations are not found on the redesigned impeller's exit. The jet-wake flow is one of very important sources undermining the diffuser pressure recovery, and consequently the stage efficiency loss and earlier stalling. 


\section{EVALUATIONS OF THE INVERSELY DESIGNED IMPELLER}
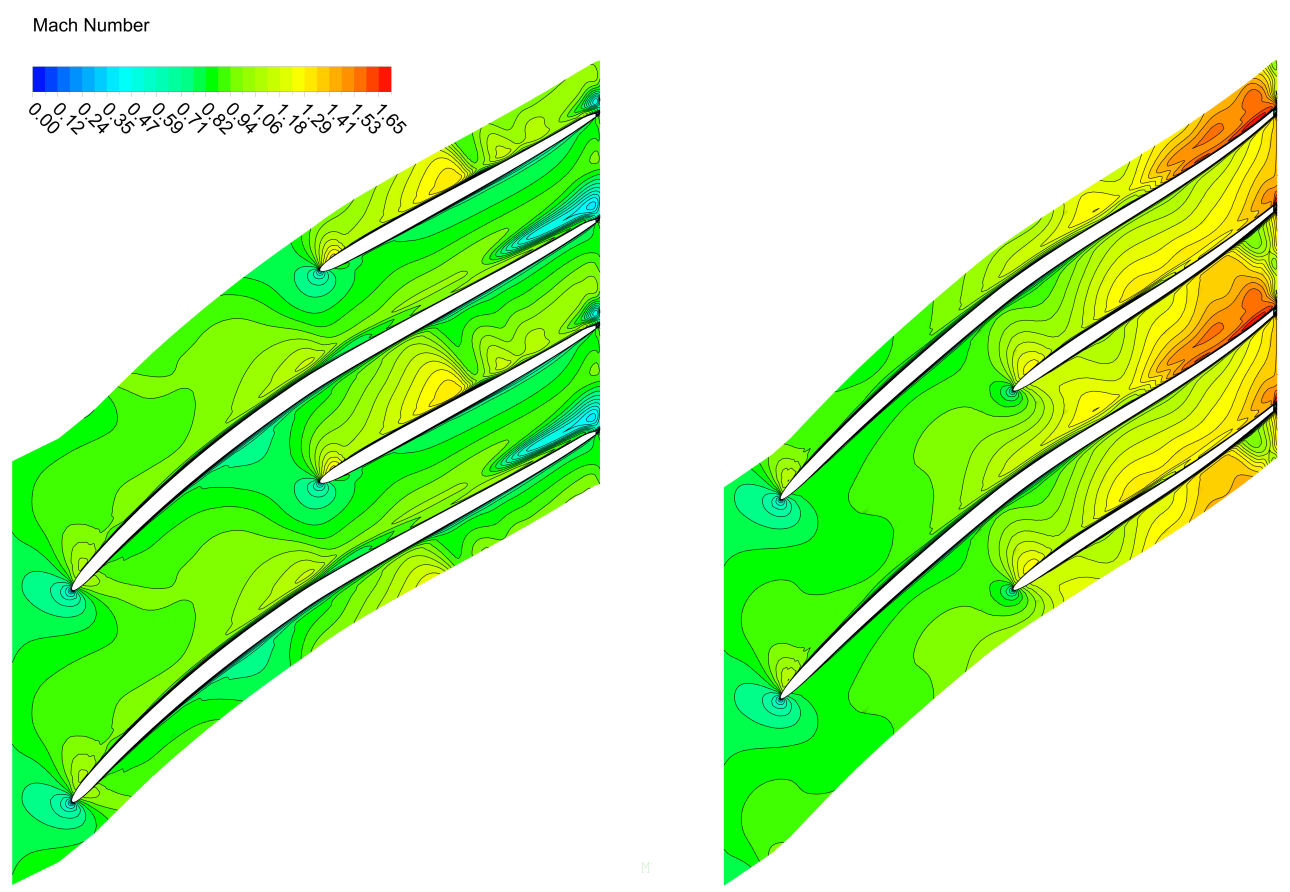

Figure 8.22: Comparisons of the relative Mach number distribution between the baseline (left) and redesign (right) impeller at the midspan at speedline 1500

\subsubsection{Shock wave structures}

The behaviour of the shock waves in the leading edge are quite distinct between the two designs which can be seen in Figs 8.37 to 8.40. The detailed Mach number distributions at different spanwise locations near the LE are compared at the BEP for both designs. Figure 8.37 compares the Mach number distributions near the leading edges of two impellers. Strong and very similar shocks can be found on both designs close to the shroud. A noticeable difference can be seen in a region that is close to the shroud on the pressure side, where the shock strength of the baseline is stronger than the redesign. This can be cross-checked with Fig. 8.40, on which a stronger shock wave on the pressure side of the baseline is clearly visible. Figure 8.38 shows very small differences of the shock wave structure and strength near the hub of both designs. Slightly more concentrated shocks are stacked in the baseline's leading edge.

Figure 8.39 presents the comparisons at the midspan of two impeller blades. Following by the two similar normal shock waves on the suction side of the in- 


\section{EVALUATIONS OF THE INVERSELY DESIGNED IMPELLER}
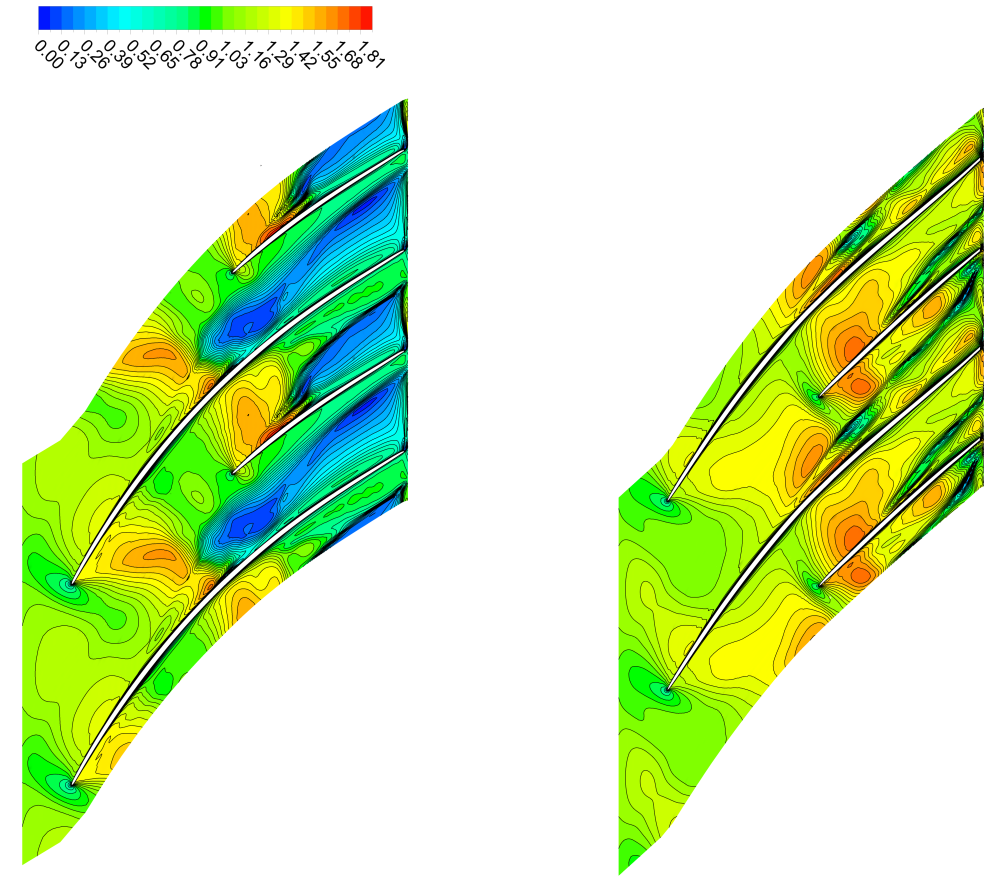

Figure 8.23: Comparisons of the relative Mach number distribution between the baseline (left) and redesign (right) impeller near the shroud at speedline 1500

ducer, there are "a street of shocks" generated on the suction side of the baseline full blade and they are extended until the exducer of the blade. This street of shocks has not been generated on the redesigned blade. It is interesting to cross-check Fig. 8.39 with Fig. 8.42, on which we can find that a very "bumpy" pressure is distributed on the suction side of the baseline full blade whereas it is much smoother on the same positions of the redesign. It should also be noted that a stronger shock is formed on the pressure side of the baseline full blade and it extends much more further towards the suction side of the adjacent blade when compared with the redesign.

Figure 8.40 compares the Mach number on a plane close to the shroud. Both designs produce a bow shock at their leading edges. Downstream of the redesigned impeller channel, a passage shock is formed on the suction surface. This sort of passage shock is also generated in the baseline design but it appears to be weaker, which can be better understood from Fig. 8.43. It is obvious that the big pressure variation on the redesigned impeller appears nearer the blade leading edge. 


\section{EVALUATIONS OF THE INVERSELY DESIGNED IMPELLER}
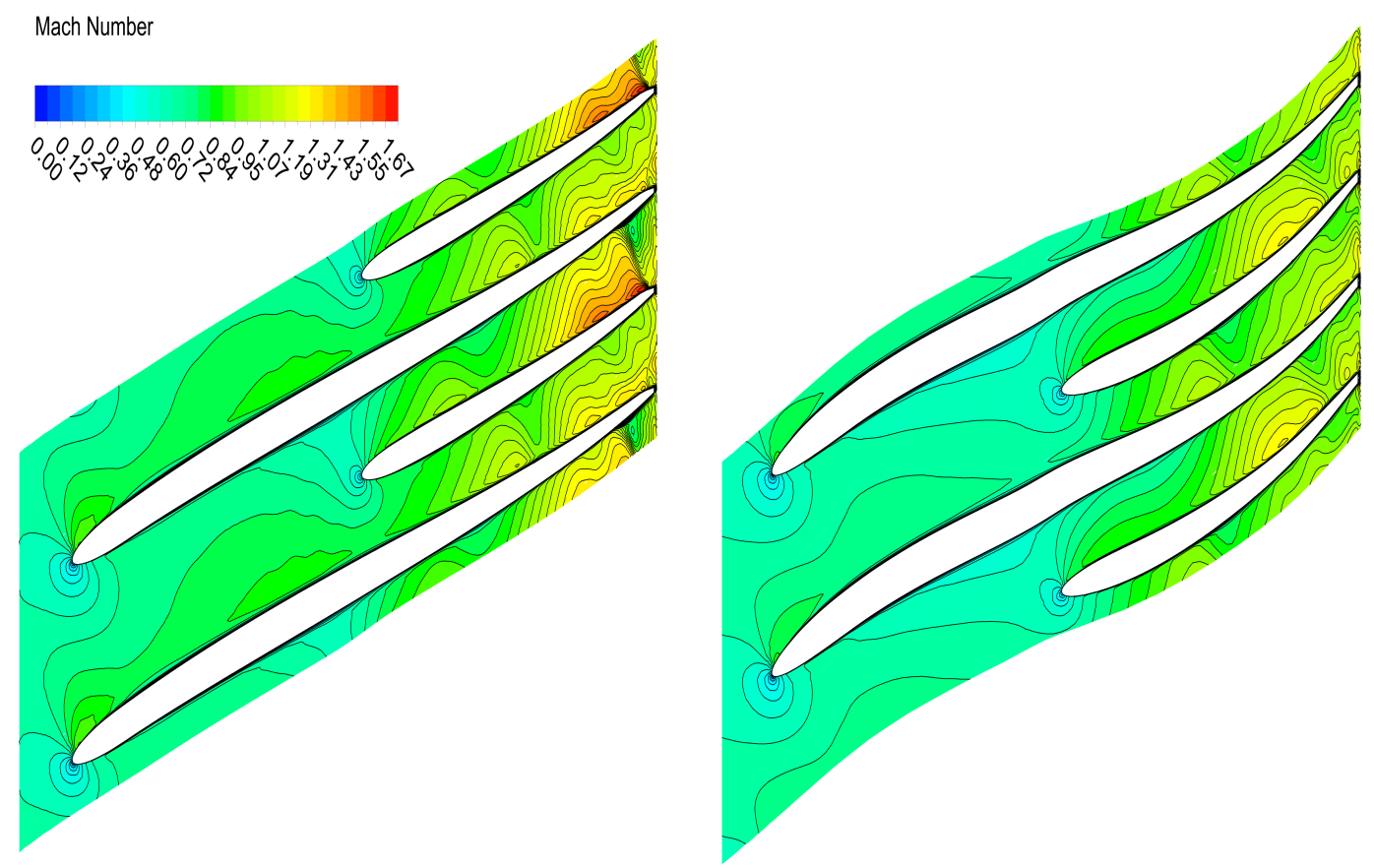

Figure 8.24: Comparisons of the relative Mach number distribution between the baseline (left) and redesign (right) impeller near the hub at speedline 1800

It is interesting to notice that a non-breaking tip leakage flow is generated right behind the passage shock in the baseline design, which propagates downstream until hitting the pressure side of the adjacent full blade. Hah et al. [2004] also noticed this phenomenon in an axial transonic compressor, and concluded that this extending and non-breaking tip leakage vortex can initiate the stall inception, which is also verified in the current work too. 

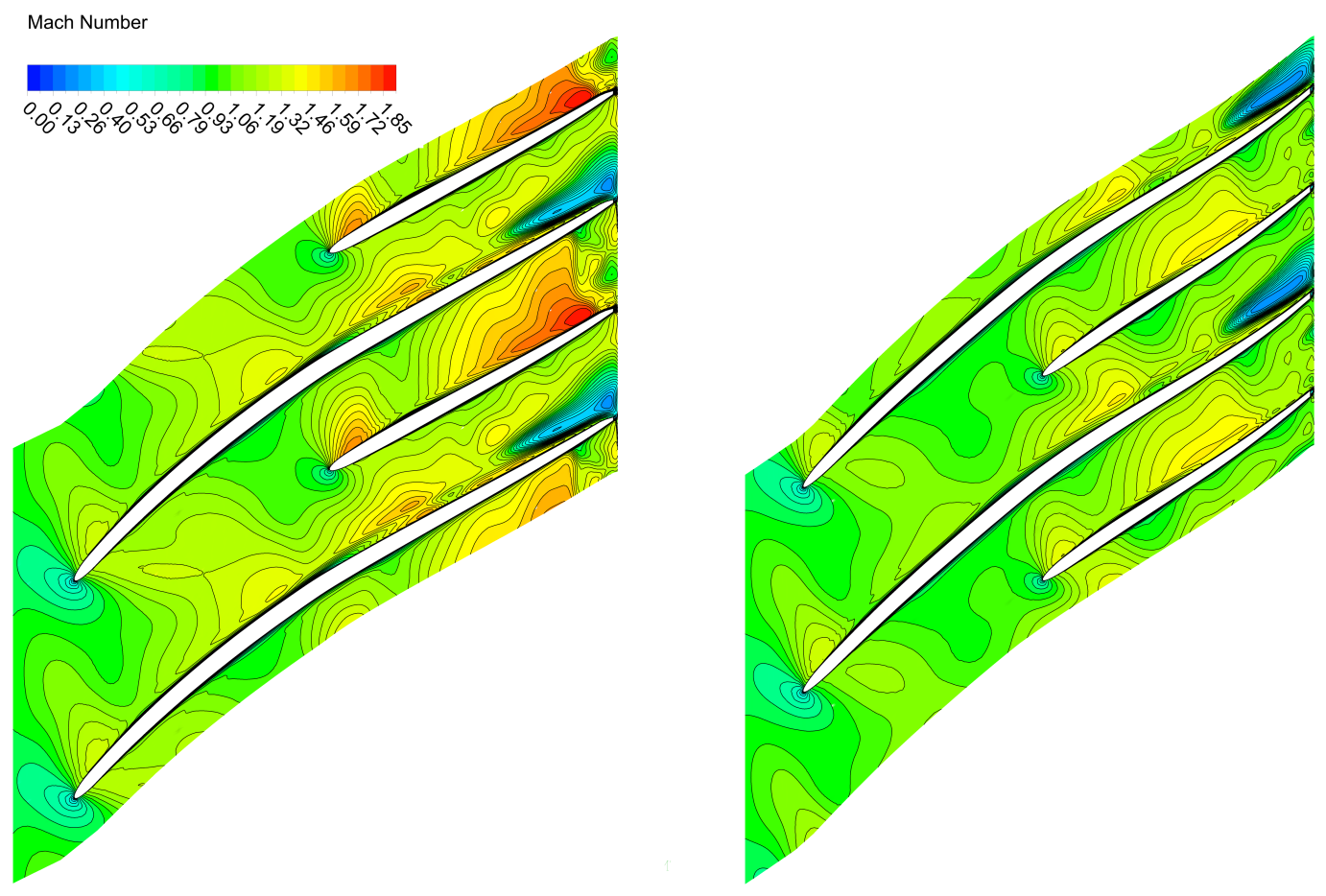

Figure 8.25: Comparisons of the relative Mach number distribution between the baseline (left) and redesign (right) impeller at the midspan at speedline 1800 


\section{EVALUATIONS OF THE INVERSELY DESIGNED IMPELLER}
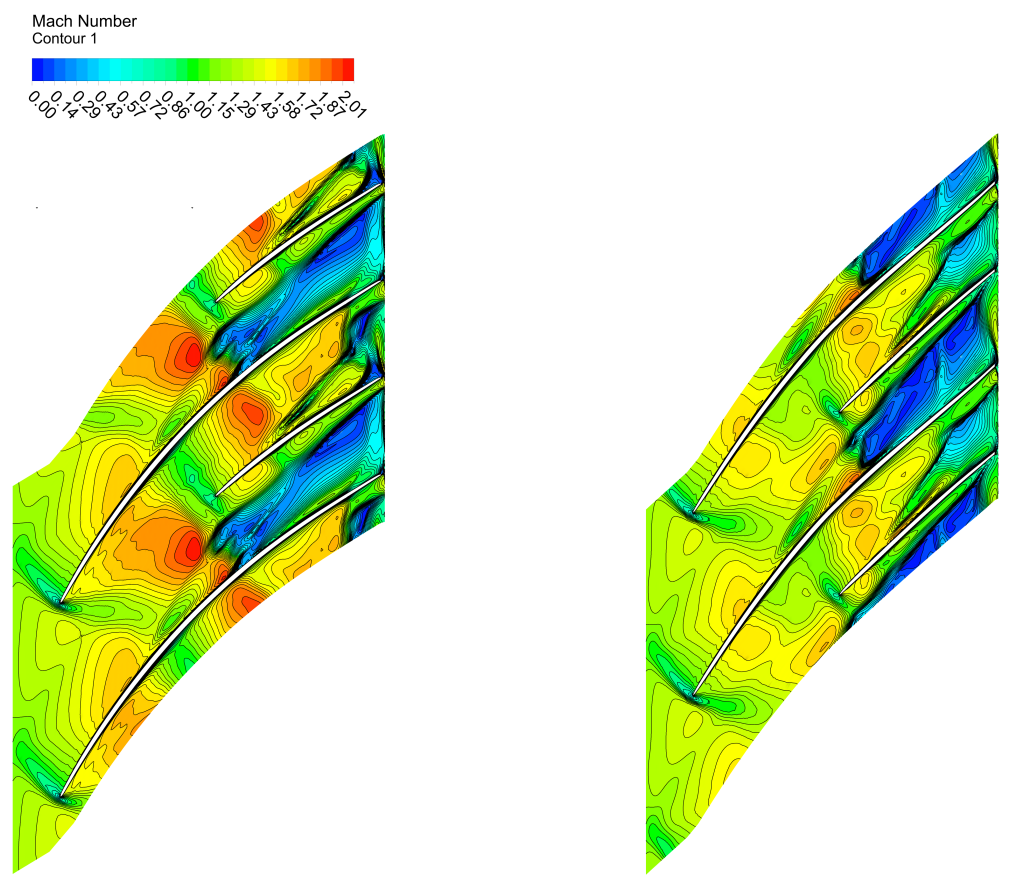

Figure 8.26: Comparisons of the relative Mach number distribution between the baseline (left) and redesign (right) impeller near the shroud at speedline 1800

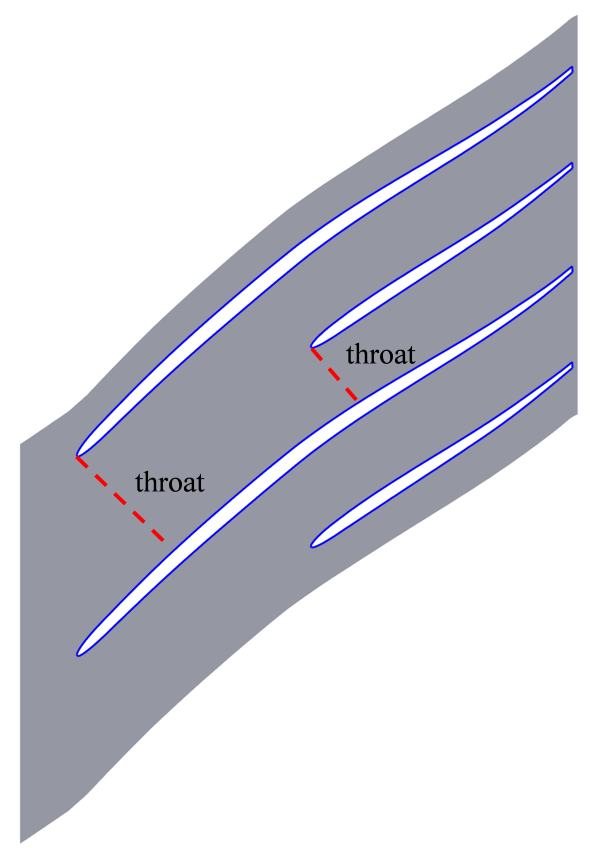

Figure 8.27: The conventional throat area schematic 
8. EVALUATIONS OF THE INVERSELY DESIGNED IMPELLER
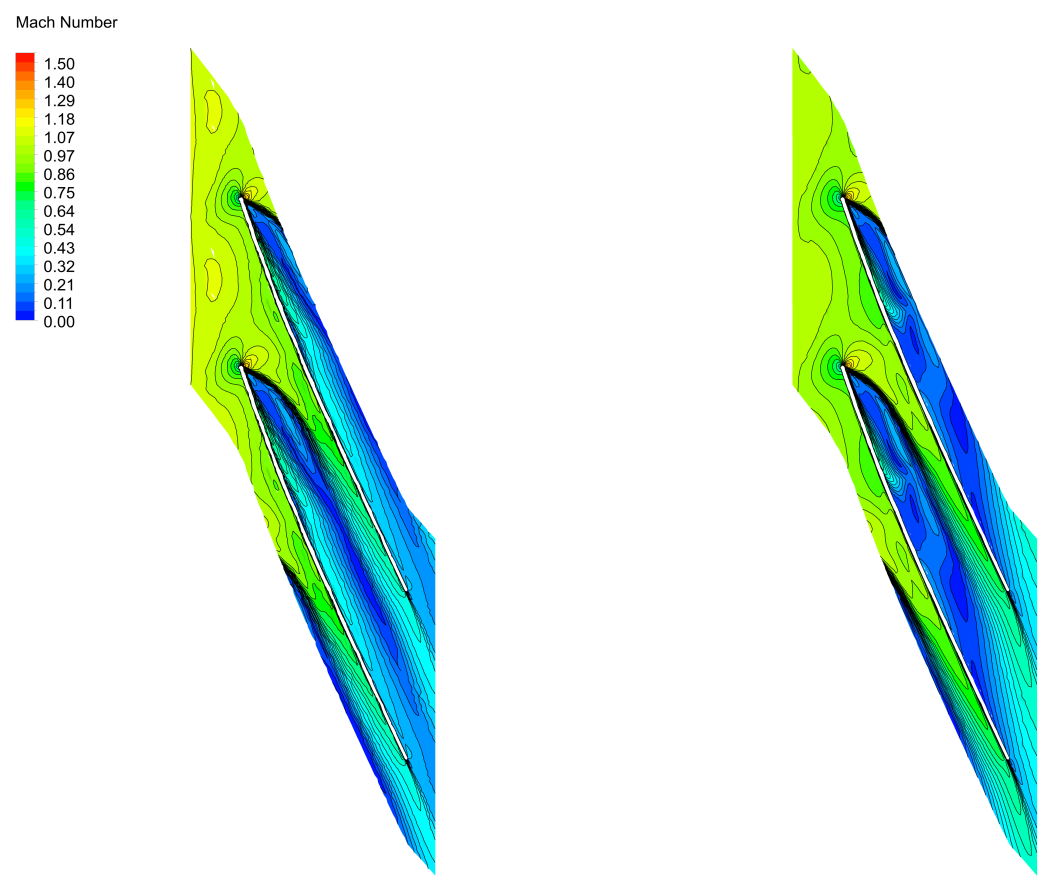

Figure 8.28: Comparisons of the relative Mach number distribution between the baseline (left) and redesign (right) near the diffuser hub at speedline 1800 

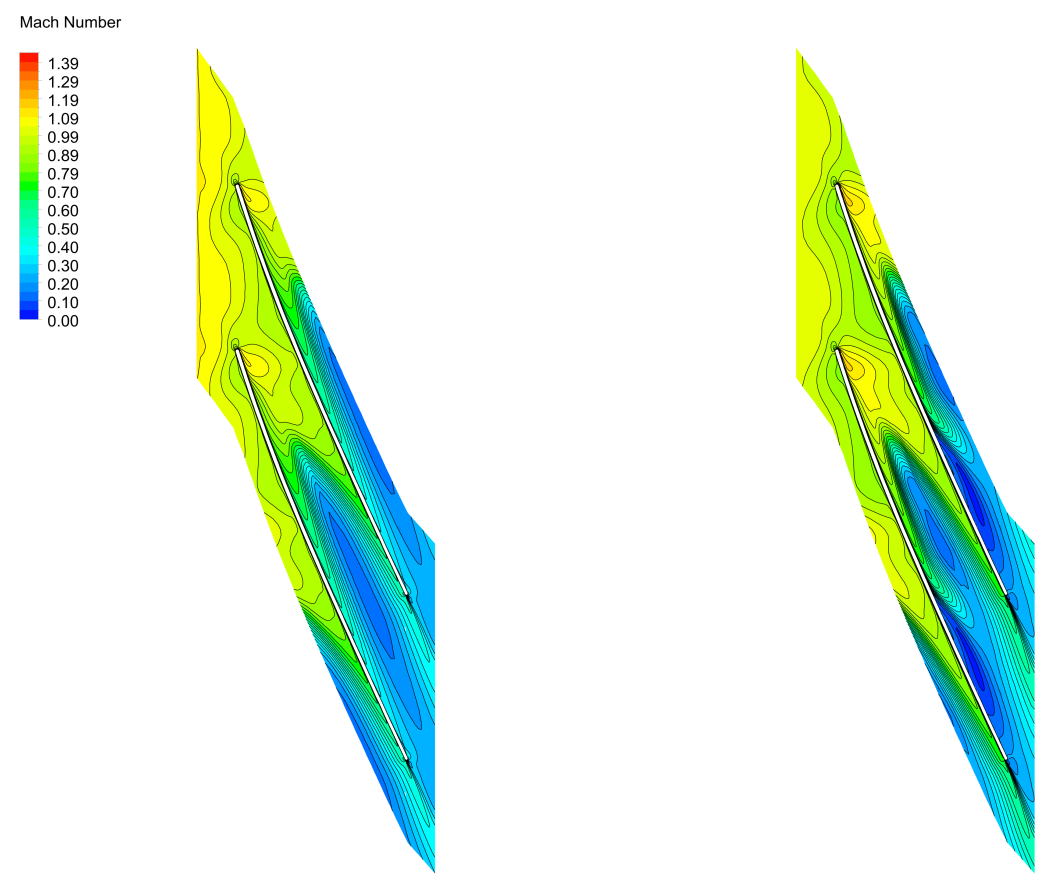

Figure 8.29: Comparisons of the relative Mach number distribution between the baseline (left) and redesign (right) at the diffuser midspan at speedline 1800 

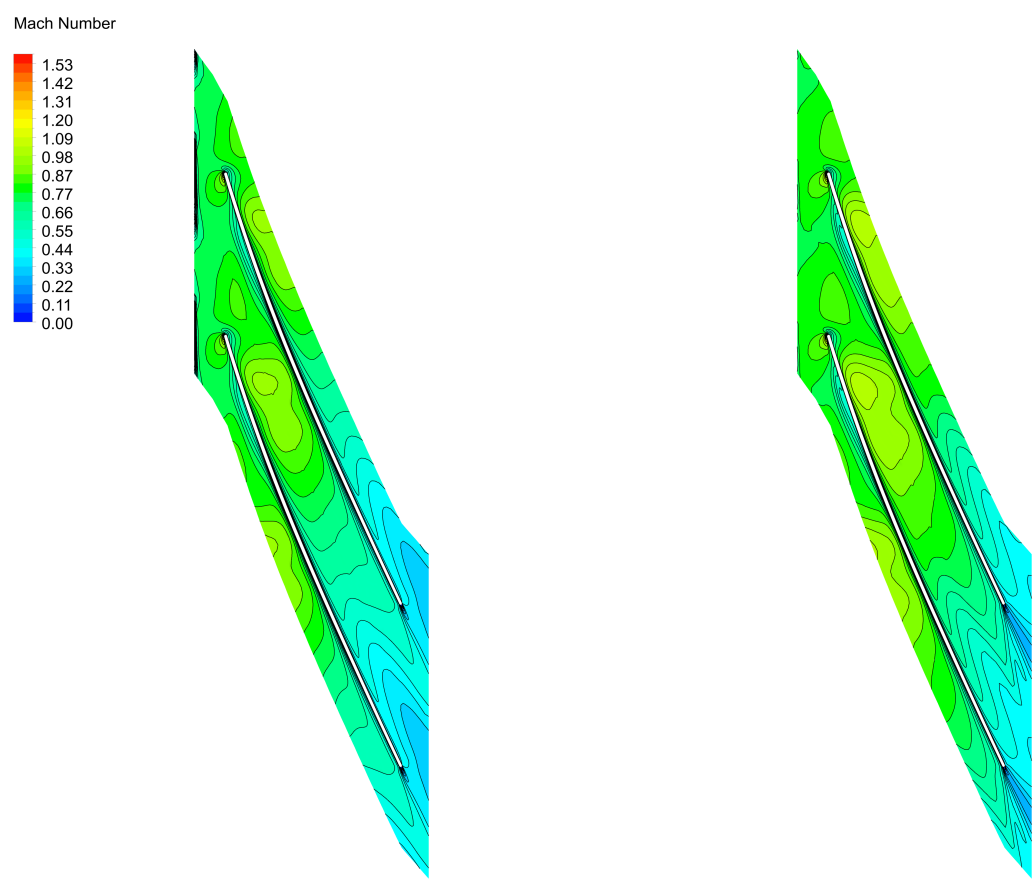

Figure 8.30: Comparisons of the relative Mach number distribution between the baseline (left) and redesign (right) near the shroud at speedline 1800
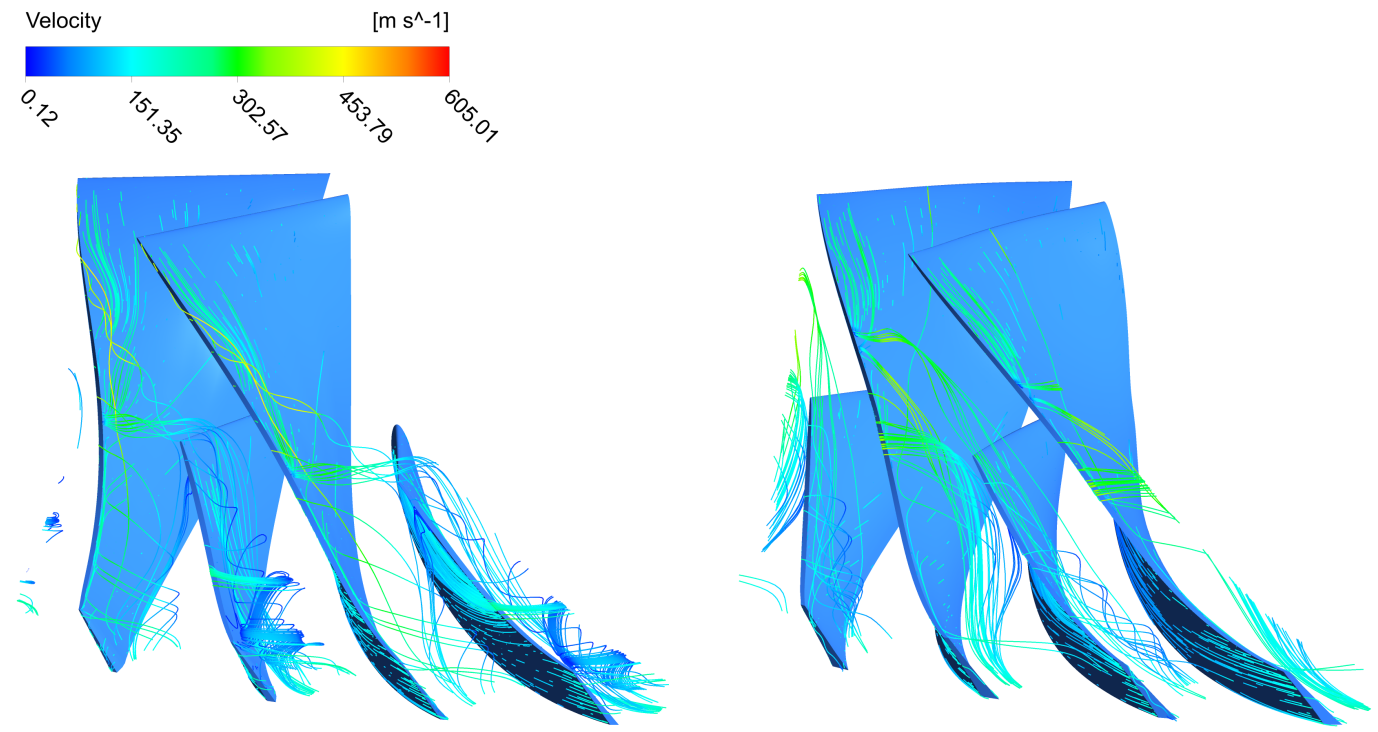

Figure 8.31: Tip leakage flow behaviours for the baseline (left) and redesign (right) blade channels at the peak efficiency point 

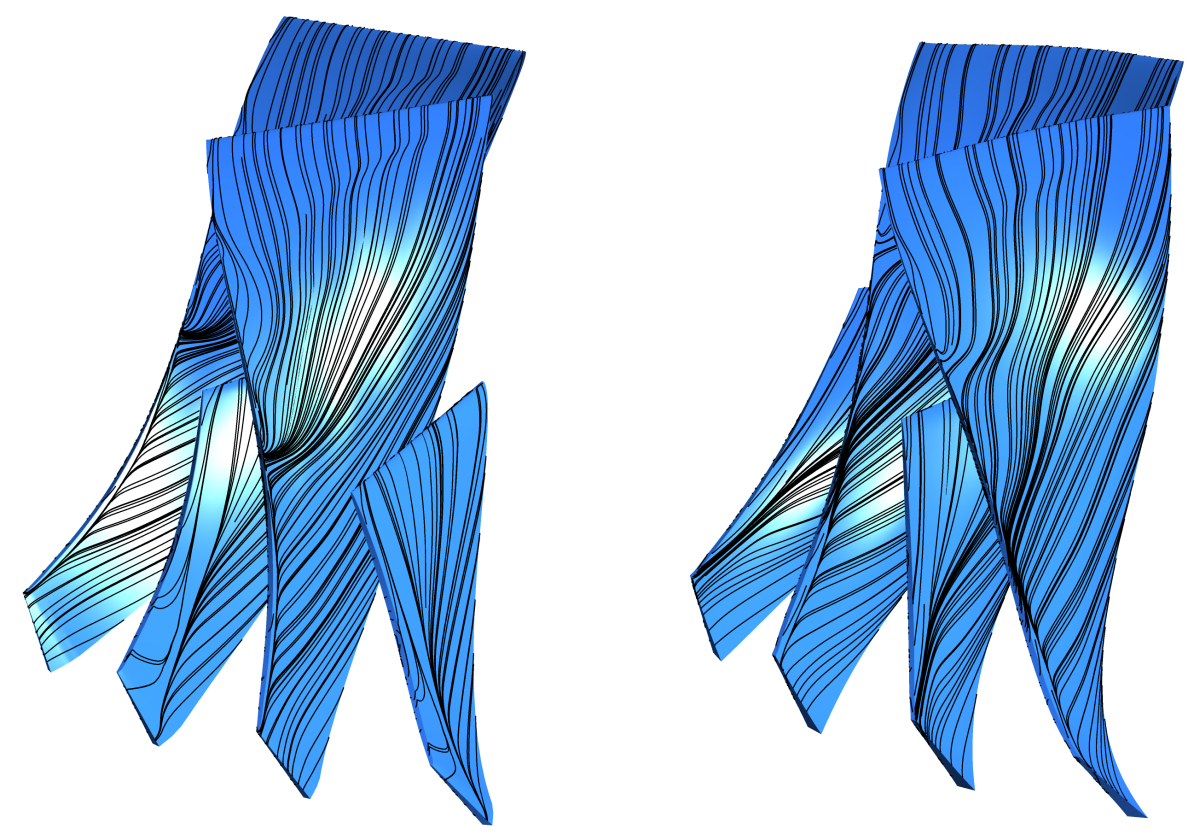

Figure 8.32: Surface streamline structures on the baseline and redesign impeller at the peak efficiency point 


\section{EVALUATIONS OF THE INVERSELY DESIGNED IMPELLER}
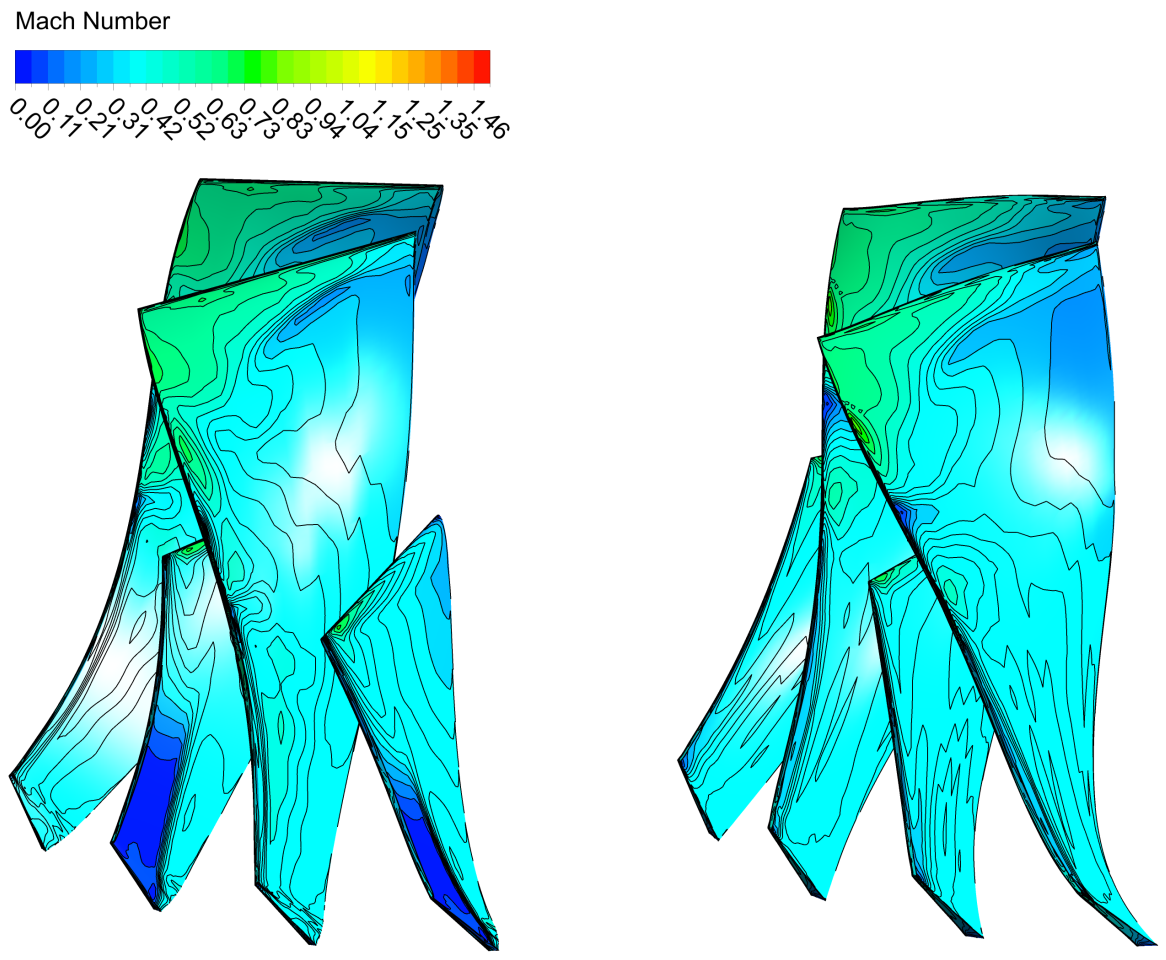

Figure 8.33: Mach number distributions on the suction surface of baseline and redesign impeller at the peak efficiency point

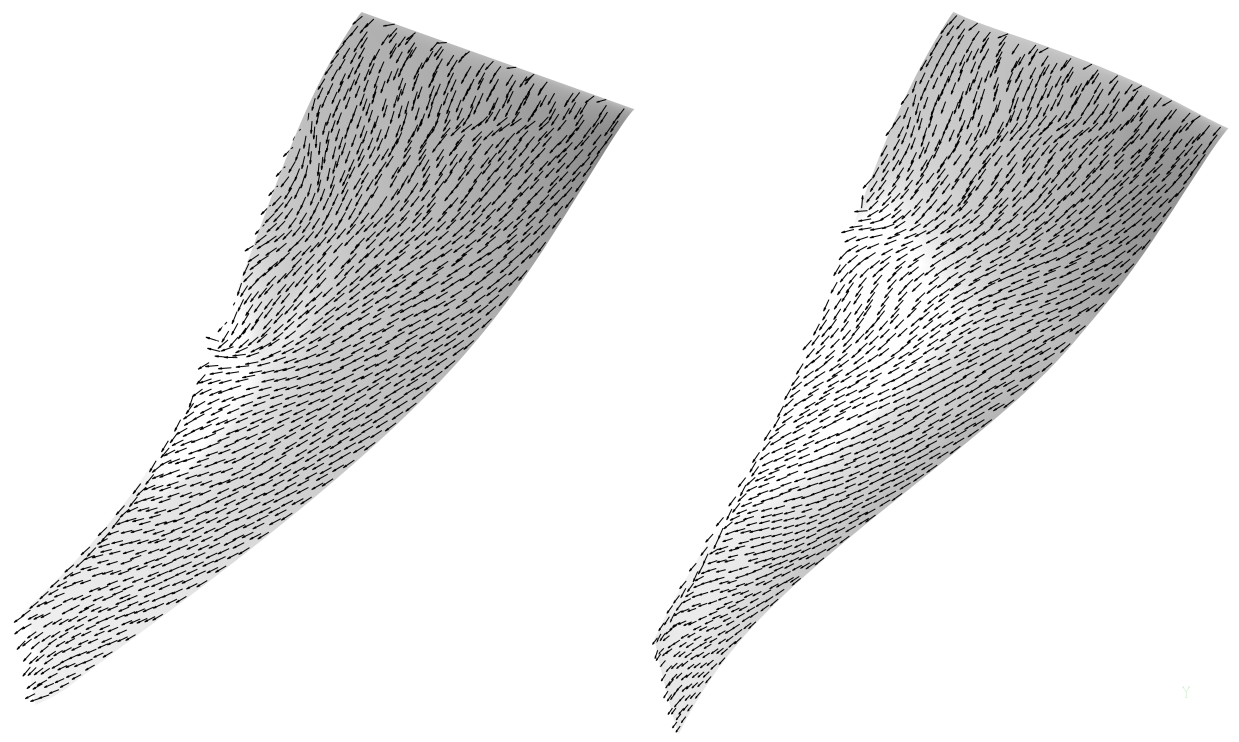

Figure 8.34: Comparisons of velocity vectors on the full blade suction surface between the baseline (left) and redesign (right) 


\section{EVALUATIONS OF THE INVERSELY DESIGNED IMPELLER}
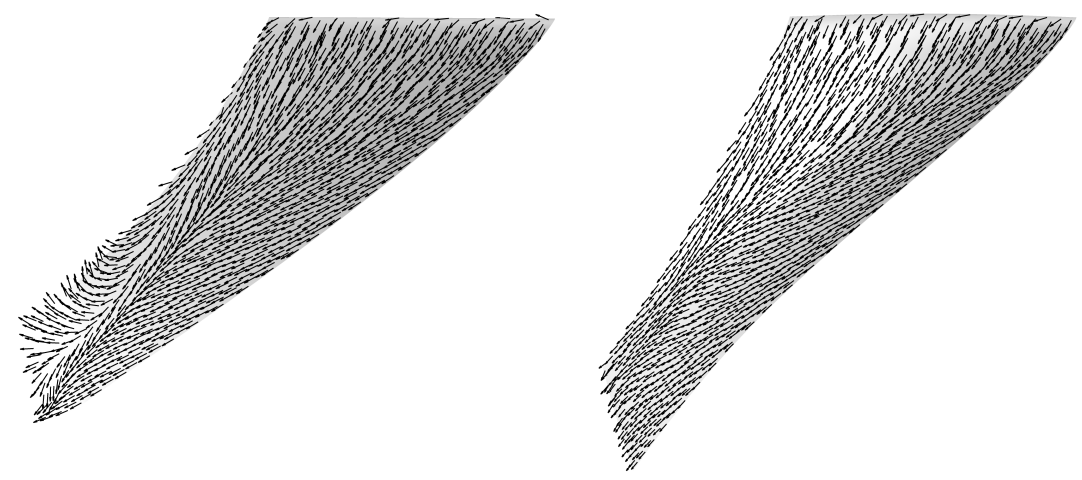

Figure 8.35: Comparisons of velocity vectors on the splitter blade suction surface between the baseline (left) and redesign (right)

Mach Number

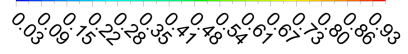
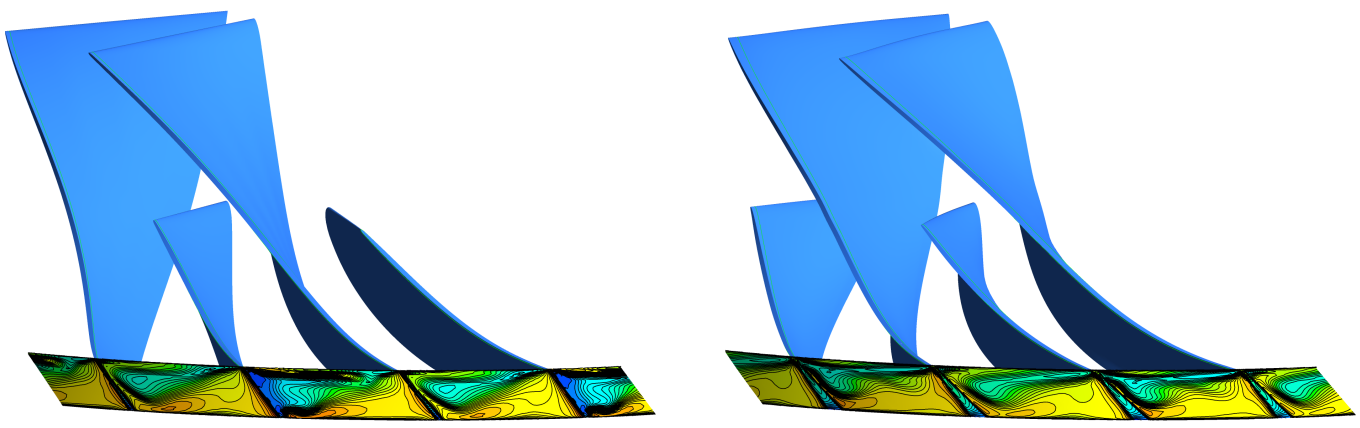

Figure 8.36: Comparisons of the exit flow Mach number at $1 \mathrm{~mm}$ downstream of trailing edge for the baseline (left) and redesign (right) impeller at the peak efficiency point 


\section{EVALUATIONS OF THE INVERSELY DESIGNED IMPELLER}
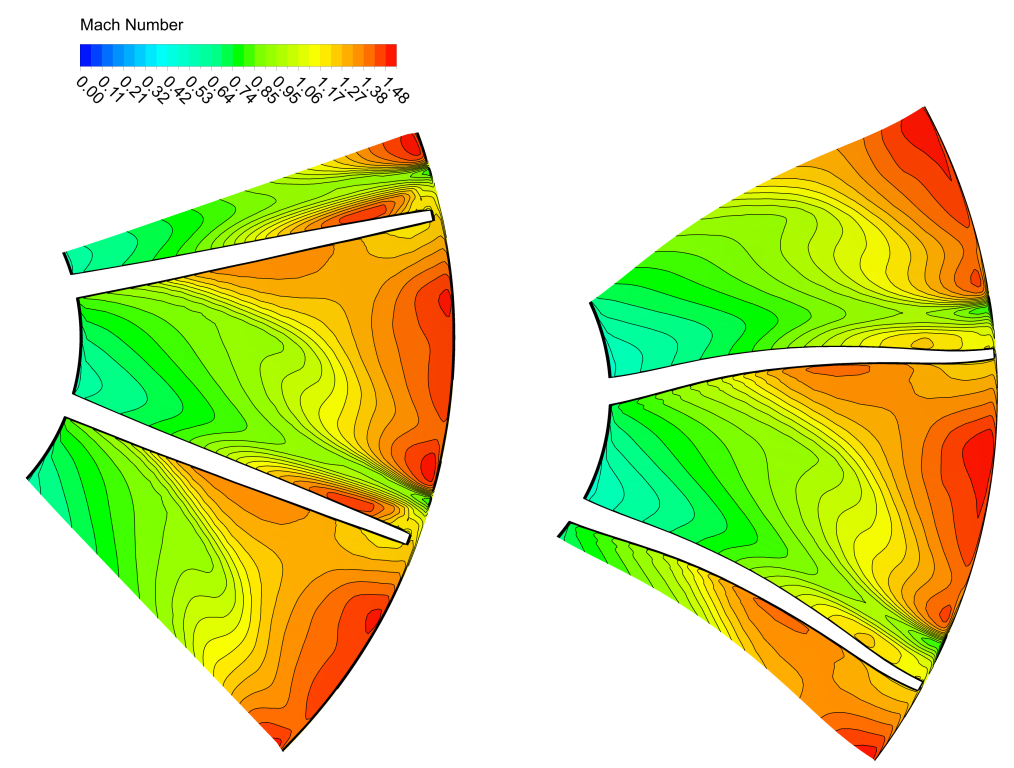

Figure 8.37: Comparisons of the relative Mach number near the impeller LE between the baseline (left) and redesign (right) impeller at the peak efficiency point
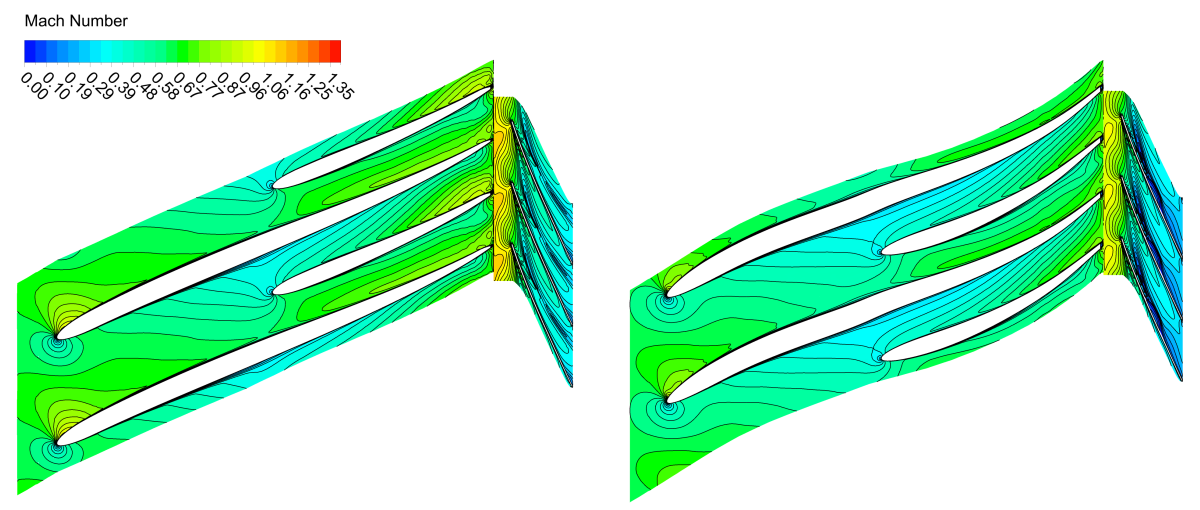

Figure 8.38: Comparisons of the relative Mach number near the hub between the baseline (left) and redesign (right) impeller at the peak efficiency point 


\section{EVALUATIONS OF THE INVERSELY DESIGNED IMPELLER}

\section{Mach Number}
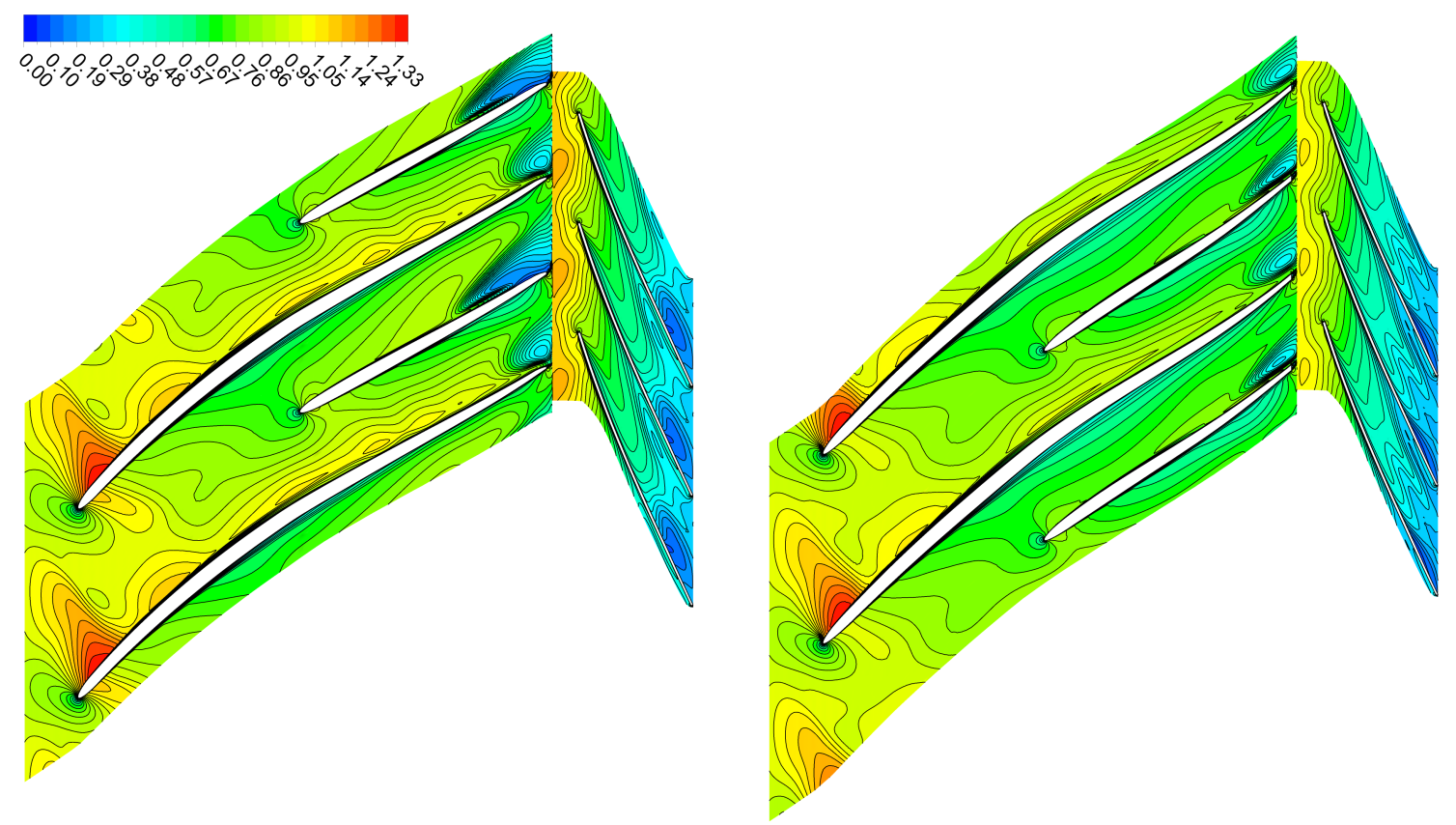

Figure 8.39: Comparisons of the relative Mach number at the midspan between the baseline (left) and redesign (right) impeller at the peak efficiency point 


\section{EVALUATIONS OF THE INVERSELY DESIGNED IMPELLER}
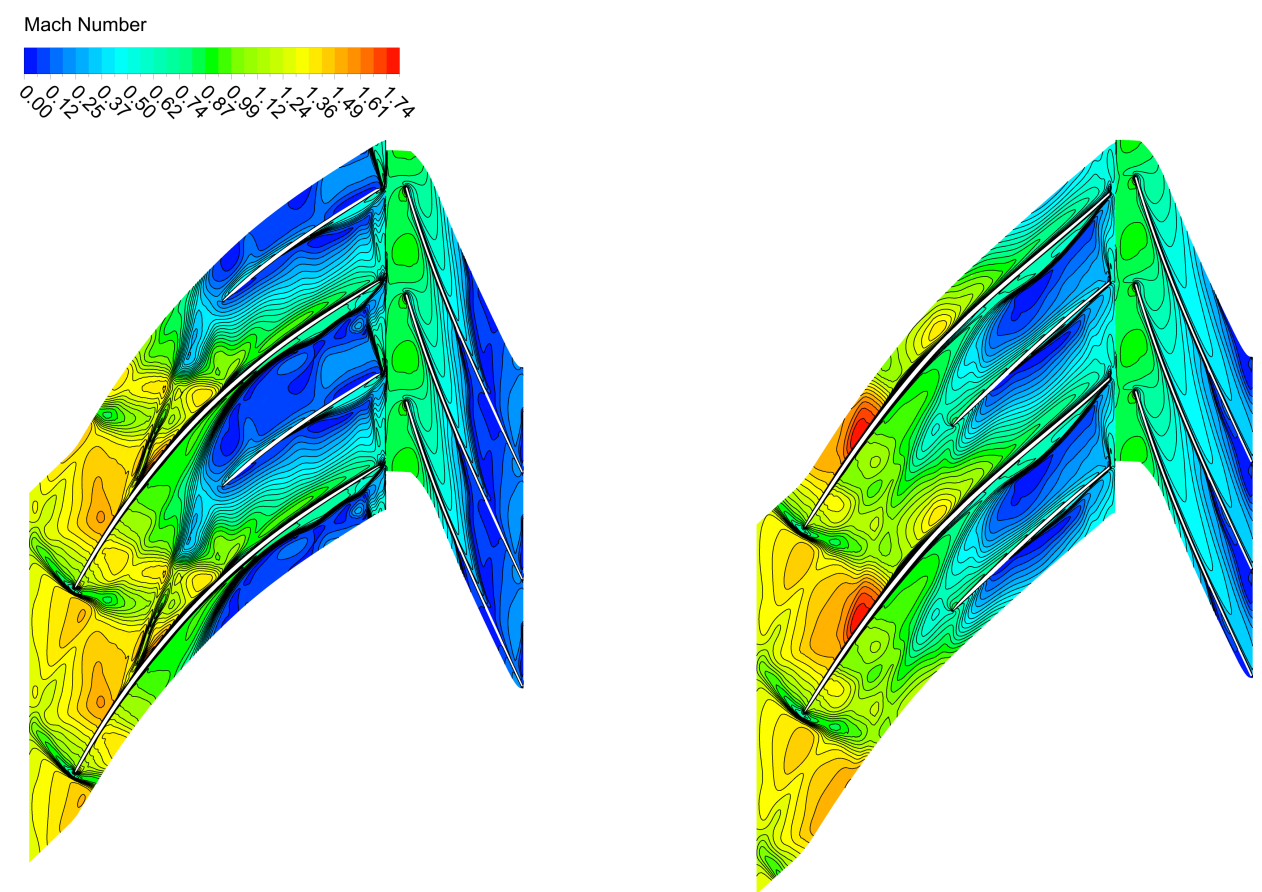

Figure 8.40: Comparisons of the relative Mach number near the shroud between the baseline (left) and redesign (right) impeller at the peak efficiency point

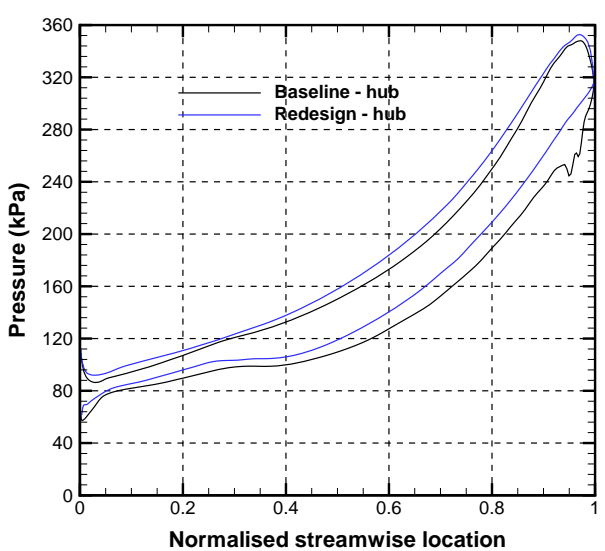

(a) Full blade

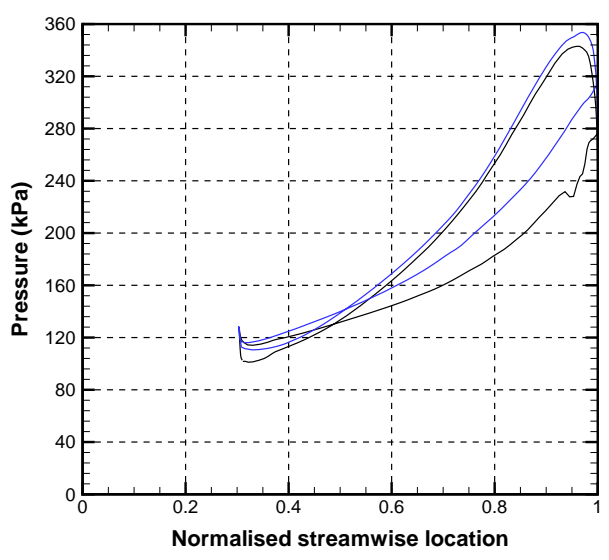

(b) Splitter blade

Figure 8.41: Comparisons of the blade surface pressure near the hub between the baseline and redesign at the peak efficiency point 


\section{EVALUATIONS OF THE INVERSELY DESIGNED IMPELLER}

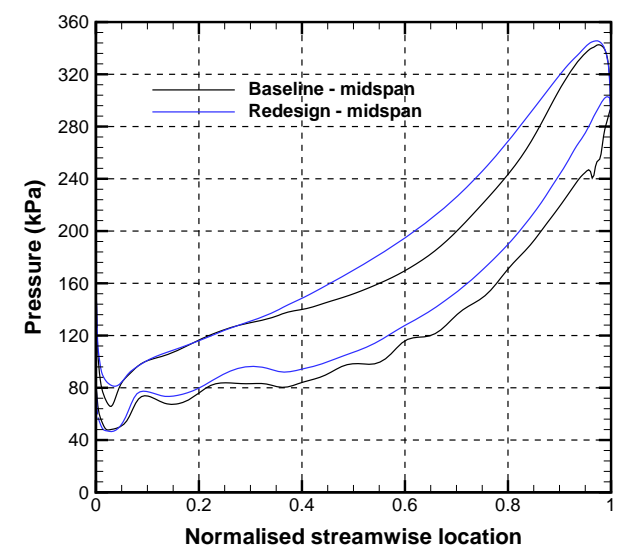

(a) Full blade

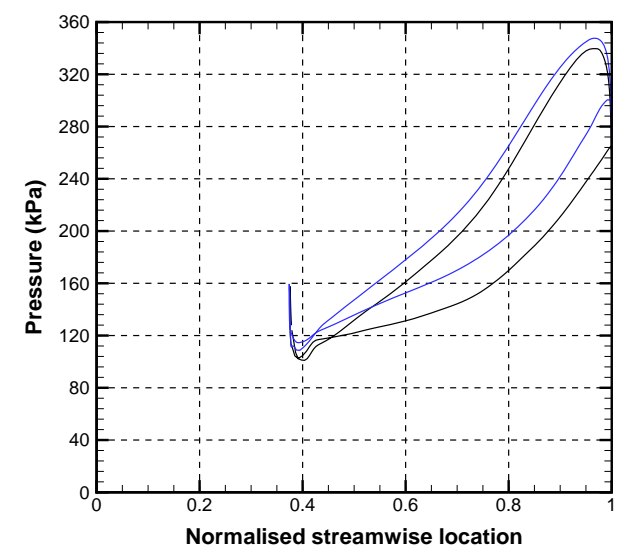

(b) Splitter blade

Figure 8.42: Comparisons of the blade surface pressure at the midspan between the baseline and redesign at the peak efficiency point

\subsubsection{Flow in the vaned diffuser}

The vaned diffuser is kept the same for both baseline and redesigns. Its leading edge has a cut-back structure with shroud leading hub that is designed to accommodate the flow from the impeller with different spanwise velocity profiles. Figure 8.44 shows the pressure recovery of the flow through this diffuser coupled with the baseline and redesigned impellers. One can see that the same vaned diffuser actually is able to achieve a much higher pressure recovery when it is used with the redesigned impeller, which can be explained by comparing the detailed flow field.

Figure 8.45 shows the Mach number in the plane slightly offset from the diffuser's leading edge. The exit flow from the baseline impeller has much higher Mach numbers that will cause strong impeller-diffuser interactions, as a result, the compressor operating range and efficiency must be reduced. Figure 8.46 compares the 3D streamlines in the diffuser channel. It can be observed that in the mid height of the diffuser, there is much more flow rolling to form vortex that moves from pressure to suction side in the baseline channel. From Figs. 8.38 to 8.40 one can also easily see the flow separations on the baseline design channel are much more severe than the redesign impeller from the hub to shroud. 


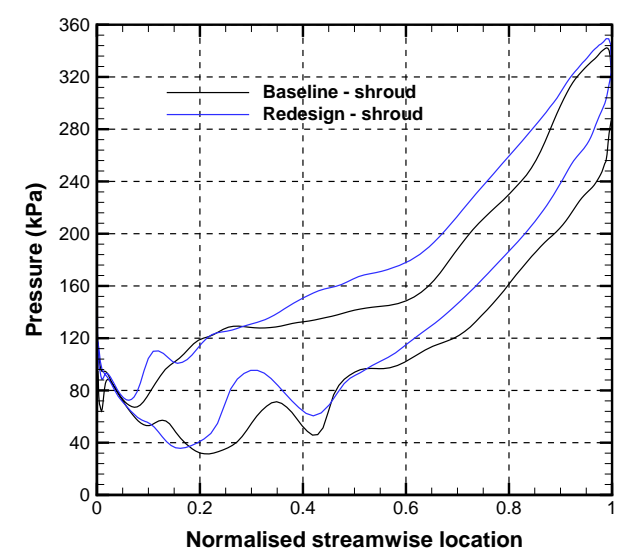

(a) Full blade

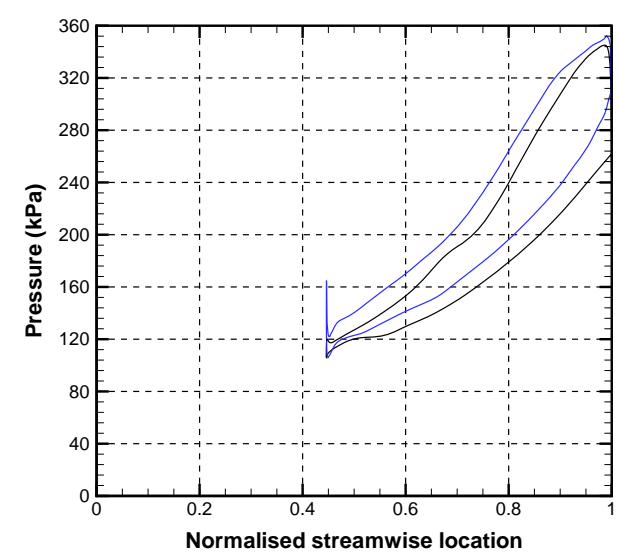

(b) Splitter blade

Figure 8.43: Comparisons of the blade surface pressure near the shroud between the baseline and redesign at the peak efficiency point

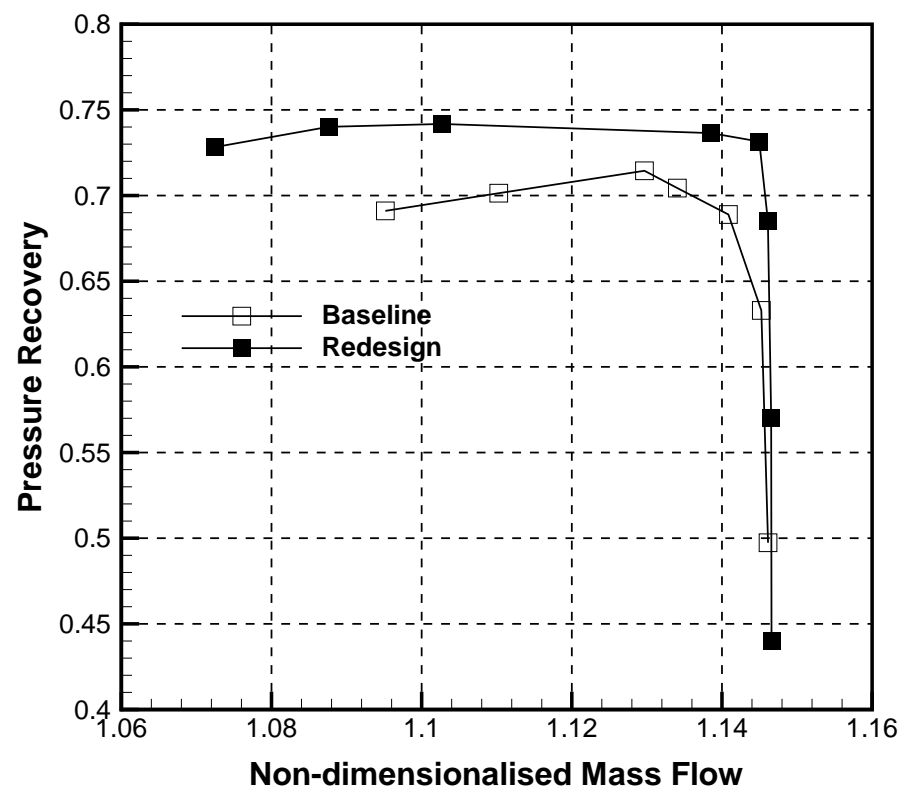

Figure 8.44: Comparisons of the pressure recovery of the same vaned diffuser when matched with the baseline and redesigned impellers 


\section{EVALUATIONS OF THE INVERSELY DESIGNED IMPELLER}
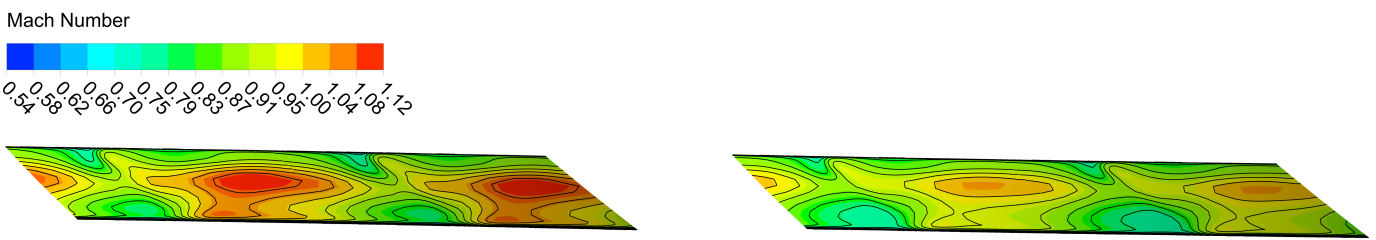

Figure 8.45: Comparisons of the Mach number at the diffuser inlet between the baseline (left) and redesign (right) impeller
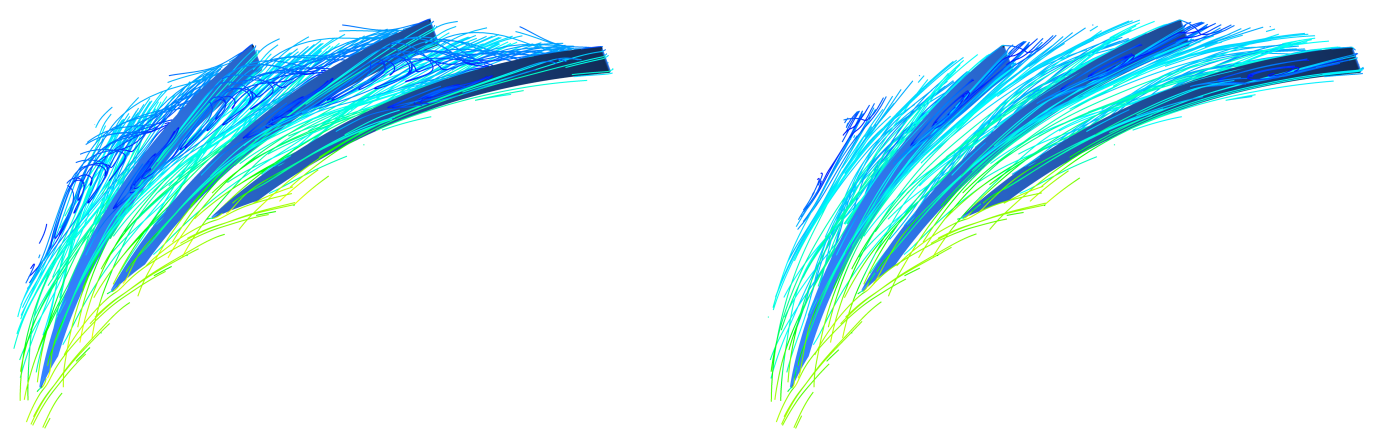

Figure 8.46: Comparisons of 3D streamlines in the same diffuser channel with incoming flow from baseline (left) and redesign (right) impeller exit

\subsubsection{Stall margins}

For a centrifugal compressor design, the wide operating range is always desired. Figure 8.7 shows the considerable advantages of the redesigned compressor's stall margin over the baseline at higher operating speeds (when the speedline is greater than 1600), which is exactly one of the design targets to achieve. Part of the mechanisms of the improved stall margins has been demonstrated in the previous paragraphs. For example, the suppressed tip leakage flow and reduced flow separations both account for the improvement, as are shown in Figs. 8.31 and 8.34. On the other hand, the more stabilised incoming flow to the diffuser is also a key source. A vaned diffuser is used in turbocharger compressors for its well-known capabilities to increase the pressure ratio and efficiency, but it would inevitably incur the earlier surge of a compressor. It is common to use a low solidity diffuser as trade-off between the aerodynamic performance and operating ranges. But it is not the intention to replace a diffuser design in this project. The impeller has to be designed in such a way to adapt the existing diffuser, which is quite contrary to a normal design process. It should be mentioned that the mesh for 


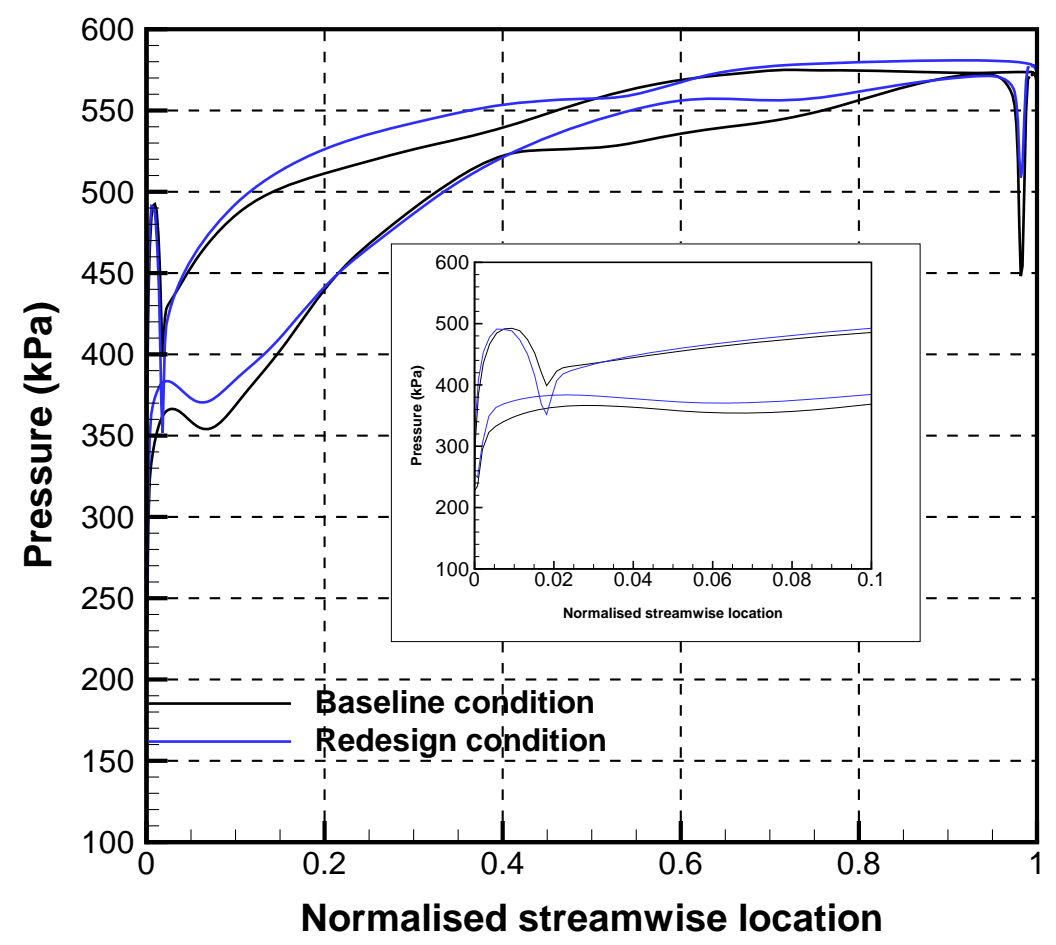

Figure 8.47: Comparisons of surface pressure on the midspan position of the diffuser with baseline and redesign incoming flow conditions

the vaned diffuser is identical for both compressor stage simulations.

Figures 8.47 show the comparisons of the surface pressure on the same diffuser subject to two different inlet flow conditions. One can see that a negative incidence is formed in the diffuser leading edge at $25 \%$ spanwise location. Several authors (Amineni et al. [1996]; Engeda [1996, 1997]) have reported that designing with a negative incidence angle for a diffuser is quite beneficial for the surge margin, and this has also been confirmed in one of the present thesis author's design projects for a racing car turbocharger. ${ }^{1}$

\subsection{Summary}

In this chapter we have endeavoured to discuss CFD and experimental results of the redesigned impeller in great detail. Fairly detailed comparisons of the

\footnotetext{
${ }^{1}$ Due to the commercial confidentiality, no details of this project can be disclosed here.
} 


\section{EVALUATIONS OF THE INVERSELY DESIGNED IMPELLER}

performances between the two designs have been given. The experimental measurements and computational calculations have both confirmed the superiority of the inversely redesigned impeller.

The CFD predictions of the redesigned compressor's characteristics were compared with the measurements and quite good agreement was obtained. Some underestimation of the choke and surge margins were due to the exclusion of the ported shroud in the CFD set up.

The characteristics of the two compressor stages were compared at 9 speedlines. The test data confirmed that the redesigned compressor shifted the high efficiency zone to the higher rpms and obtained greater surge margins for high speeds as well. The acoustic measurements for the two designs were conducted at various speeds and locations. The results showed that the inversely designed impeller can lead to significant noise level reductions at the blade passing frequency.

Following that, extensive internal flow field analyses of two designs were given, including:

- the explanation of the reduced choke margin of the new impeller at lower rpms even if its throat area is very close to the baseline;

- reduced tip leakage flows from the redesign indicating an enhanced surge margin can be obtained;

- improved flow uniformity at the exit of redesigned impeller that would lead to fewer losses in the vaned diffuser and hence a better stage performance;

- weakened shock strength at the new impeller's leading edge that would contribute to both aerodynamic performance improvement and reduction of BPF tones;

- improved flow behaviours in the vaned diffuser that gives higher pressure recovery;

- enhanced surge margins at high speeds due to reduced tip leakage flows and negative incidence angle at the diffuser vane's leading edge. 


\section{EVALUATIONS OF THE INVERSELY DESIGNED IMPELLER}

The success achieved in this compressor redesign project has provided strong evidence that the inverse design method can help to improve the turbomachinery performance greatly and shorten the design period significantly. 
"You can't connect the dots looking forward; you can only connect them looking backward."

Steve Jobs (1955 - 2011)

"Things won are done; joy's soul lies in the doing."

William Shakespeare (1564 - 1616)

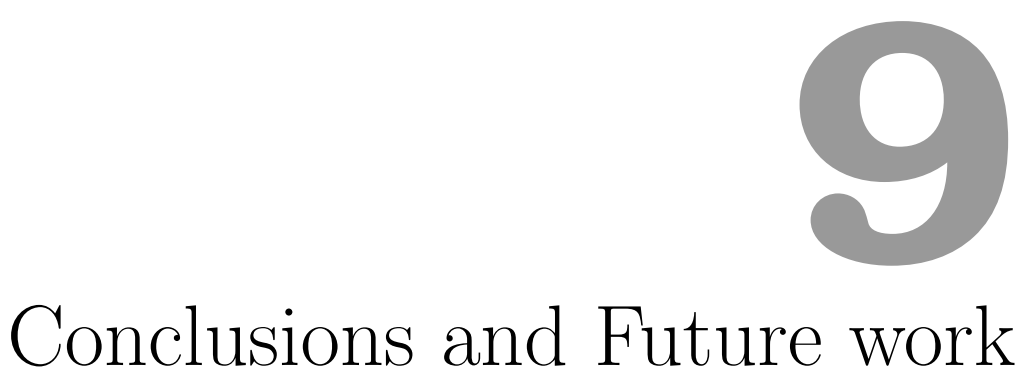

In this thesis we have presented a multi-objective and multi-disciplinary study for a transonic centrifugal compressor used for marine turbochargers. Numerical calculations of acoustics, CFD predictions of compressor characteristics, aerodynamic analyses of flow field, inverse design, FEA analyses, and experimental validations have all been attempted. An inversely designed impeller wheel has been prototyped by and tested at Napier Turbochargers Ltd. The measured data show that the inversely designed compressor has significant advantages at the desired speed lines over the baseline impeller in terms of aerodynamic efficiencies and aeroacoustic noise generations. Apart from the aero performances, the FEA modelling also confirmed that the peak stress and total deformation of the redesigned blades are both lower than the baseline whereas the vibration frequency at all different modes are higher than the baseline. This chapter is dedicated to summarising the accomplishments achieved in this thesis and proposing the possible aspects for future research that are of interest and significance for the turbomachinery community. 


\subsection{Accomplishments achieved in this thesis}

- Reviewed, from the first principle, most of the important literature on the aeroacoustic theories and methods for rotor-induced noise.

- Developed a set of mathematical formulations that can be used for the rotor noise modellings starting from the fundamental Lighthill acoustic analogy and FW-H equation.

- Developed an aeroacoustic code that can be used for the subsonic and transonic rotor noise predictions. The detailed implementation algorithms and manuals are both presented. The noise induced by the UH1H helicopter rotor with NACA 0012 as the cross-section shape and DLR's well-known SRV2 impeller were computed and validated with the published data in literatures.

- Derived the formulations of the inverse design method that can be used for the design of multiple blades per channel.

- Fully analysed an existing transonic compressor stage used in marine turbochargers with CFD simulations.

- Redesigned an impeller using the 3D inviscid inverse design method with improved aerodynamic, aeroacoustic and mechanical performances.

- Acoustic and aerodynamic measurements were carried out for both impellers which confirmed the new impeller's advantages.

\subsection{Recommendation of future work}

In this section we delineate the framework of possible future studies derived from the necessity of developing and enhancing the present thesis. We suggest from three aspects, aeroacoustics, aerodynamics, and turbomachinery design to further the current work. 


\section{CONCLUSIONS AND FUTURE WORK}

\subsubsection{Aeroacoustics}

One of the primary objectives set at the beginning is to develop an aeroacoustic solver that can be used to evaluate the compressor noise and incorporated into the inverse design method. It has been explained that the compressor's noise field is highly complicated and also time dependent. In this work, since only the steady state solutions were used for the convective FW-H equation to estimate the noise, there are only sound pressure levels at BPFs computed. The broadband components of the real noise spectrum have been neglected. Additionally, the sound propagations in the solid duct were not considered either. Including these two issues means a thorough analysis of the compressor noise in the far and near field, which demands huge amount of CFD resources. Due to the limited computational resources and time allowance of this project, we could not fully resolve the aeroacoustic field of the compressor, which is, however of great interest for the turbomachinery community. If the computational resources and good acoustic testing facilities are available, it is certainly useful to study the following aspects of the compressor aeroacoustics:

- The sound level and directivity at different operating mass flow rates and rotational speeds. Particularly, the sound mechanisms of compressor choking and stalling at subsonic and transonic conditions are worth careful examinations using test data and numerical simulations.

- The measurement of noise at the exit of the diffuser, which reveals the rotor-diffuser interaction effects on the noise field.

- The more advanced turbulence models, such as LES (large eddy simulations), can be performed and coupled with the Farassat Formulation 1B [Farassat \& Casper, 2012] for the broadband noise predictions with transient solutions.

- The boundary element method can be used to include the duct effects on the propagation of the acoustic field.

We should also remind ourselves that the purpose of understanding the noise mechanism better is to design a turbomachine with lower noise. Thus, the trade- 


\section{CONCLUSIONS AND FUTURE WORK}

off between the acoustic field calculations with high fidelity and obtaining a better impeller design should always be considered.

\subsubsection{Aerodynamics}

Although the development of CFD technology has greatly enhanced our understanding of the aerodynamics of the flow in turbomachines, particularly for the prediction of a characteristic map, the correlations between flow behaviours and compressor's different operating conditions are not understood well. For instance:

- The onset of a compressor's stall. The detailed evolutions of the flow phenomena from the choking to stalling in the blade channel can be investigated to understand what essentially triggers the compressor stall at different speedlines so that counter measures could be found.

- The quantification of losses due to shock waves, secondary flow, flow separation, and jet-wake flows. There is still lack of a viable mathematical modelling for the numerical evaluations of the compressor's loss mechanism. Our understanding on the compressor's losses are still of descriptive nature.

- Conduct the CFD simulations using the deformed blade shape. As has been pointed out, the blade will inevitably be deformed/deflected due to centrifugal forces during operations whereas most simulation literatures published so far have assumed the absolute rigidity of the blade. This is at least one of the reasons that causes the inaccuracy of CFD predictions for the compressor map, particularly for the choke margin, as a slight change of the blade profile will result in a change of throat area, consequently leading to the change of choke margin.

\subsubsection{Turbomachinery design and optimisation}

The design process used in this project is quite effective and has very good efficacy. It has been shown that adjusting the blade loading parameters, $r V_{\theta}^{*}$ and 


\section{CONCLUSIONS AND FUTURE WORK}

$\partial\left(r V_{\theta}^{*}\right) / \partial m^{*}$ can give us designs with significantly improved impellers. However, we still have not explored all possible design space, for example:

- The optimisation of the meridional contour. The hub and shroud profile can be parametrised and optimised for even better performances.

- Improve the diffuser for the new impeller wheel. In this work only the impeller blade has been redesigned and directly coupled with the existing vaned diffuser. For the optimal performances a new diffuser vane should be designed to match the new impeller.

- A volute can also be redesigned to replace the exiting one. For instance, a twin-exit volute can be possibly used for a more compact stage structure.

- Parametric studies on the control variables of the blade loadings, such as, NC, ND, Slope, DRVT to understand how exactly those variables affect the impeller's aerodynamic and aeroacoustic performances. Although this will be a very time-consuming and computationally expensive process, obtaining the correlations through such parametric studies will bring great benefits for the turbomachinery industry. A database can be established eventually to quickly get the optimal designs.

- In the current formulations for inverse design only one splitter blade is allowed. This can be easily adjusted using the formulations derived in Chapter 6 to allow more than one splitter blade to be designed. The same idea can be used in designing vaned diffusers with a long-short blade configuration.

- The full and splitter blades are in fact designed independently in the current formulations. The only connection between them is the total $r V_{\theta}^{*}$ at the impeller exit. This can lead to non-physical blade designs, for instance, the inverse solver can find a solution that the full and splitter blades are placed too close in the pitchwise direction. A constraint condition that confines the relative position between these two blades can be introduced to the current inverse design algorithm. 


\section{Bibliography}

Amiet, R.K. (1988). Thickness noise of a propeller and its relation to blade sweep. Journal of Fluid Mechanics, 192, 535-560.

Amineni, N., Engeda, A., Hohlweg, W. \& Direnzi, G. (1996). Performance of low solidity and conventional diffuser systems for centrifugal compressors. Proceedings of ASME 1996 International Gas Turbine and Aeroengine Congress and Exhibition, Birmingham, UK, June 10-13, 1996.

Arbabi, A. \& Ghaly, W. (2013). Inverse design of turbine and compressor stages using a commercial cfd program. ASME Turbo Expo 2013: Turbine Technical Conference and Exposition, GT2013-96017.

Baeder, J.D., Galllman, J.M. \& Yu, Y.H. (1997). A computational study of the aeroacoustics of rotor in hover. Journal of the American Helicopter Society, 42, 39-53.

Batchelor, G. (2000). An Introduction to Fluid Dynamics. Cambridge University Press.

Blokhintsev, D. (1956). Acoustics of a nonhomogeneous moving medium. NACA TM-1399.

Borges, J. (1990a). A three-dimensional inverse method for turbomachinery: Part I - Theory. Journal of Turbomachinery, 112(3), 346-354. 
Borges, J. (1990b). A three-dimensional inverse method for turbomachinery: Part II - Experimental verification. Journal of Turbomachinery, 112(3), 355361.

Boselli, P. (2016). An inverse design methodology for long last-stage steam turbine blades. University College London.

Boxwell, D.A., Yu, Y.H. \& Schmitz, F.H. (1979). Hovering impulsive noise: some measured and calculated results. Vertica, 3(1), 35-45.

Brentner, K.S. (1994). A new algorithm for computing acoustic integrals. Proceedings of IMACS 14th World Congress on Computational and Applied Mathematics, 2, 592-594.

Brentner, K.S. \& FArassat, F. (2003). Modelling aerodynamically generated sound of helicopter rotors. Progress in Aerospace Sciences, 39, 83-120.

Bres, G.A., Brentner, K.S., Pereza, G. \& Jonesb, H.E. (2004). Maneuvering rotorcraft noise prediction. Journal of Sound and Vibration, 275, 719-738.

Brigham, E.O. (1974). The Fast Fourier Transform. Prentice-Hall, Inc., Englewood Cliffs, N.J., USA.

Carolus, T., Zhu, T. \& Strum, M. (2015). A low pressure axial fan for benchmarking prediction methods for aerodynamic performance and sound. Fan2015, Lyon(France), 15- 17, April 2015.

Casalino, D. (1996). Aeroacoustics of 2-d and 3-d blade vortex interaction using the indicial method. Proc. 52nd Annual Forum American Helicopter Society, Washington, DC: American Helicopter Society.

Casalino, D. (2003). An advanced time approach for acoustic analogy predictions. Journal of Sound and Vibration, 261, 583-612.

Chen, H. \& LeI, V. (2013). Casing treatment and inlet swirl of centrifugal compressors. Journal of Turbomachinery, 135(4). 
Cheney, W. \& Kincaid, D. (2008). Numerical mathematics and computing. Bob Pirtle, USA, 6th edn.

Cumpsty, N.A. (1977). Critical review of turbomachinery noise. Trans ASME Journal of Fluids Engineering, 99, 278-293.

Cumpsty, N.A. (1989). Compressor Aerodynamics. Longman Scientific and Technical, New York.

Curle, N. (1955). The influence of solid boudaries upon aerodynamic sound. Proceedings of the Royal Society of London, Series A, Mathematical and Physical Sciences, 231(1187), 505-514.

DAng, T. \& IsGro., V. (1995). Euler-based inverse method for turbomachine blades part 1: Two-dimensional cascades. AIAA, 33(12), 2309-2315.

Dang, T., Damle, S. \& QIU, X. (2000). Euler-based inverse method for turbomachine blades part2: Three-dimensional flows. AIAA, 38(11), 2007-2013.

Demeulenaere, A. \& Den Braembussche, R.V. (1998). Three-dimensional inverse method for turbomachinery blading design. Journal of Turbomachinery, 120, 248-255.

Demming, A.F. (1938). Noise from propellers with symmetrical sections at zero blade angle. NACA, TM 769.

di Francescantonio, P. (1997). A new boundary integral formulation for the prediction of sound radiation. Journal of Sound and Vibration, 202(4), 491509.

Dixon, S.L. \& Hall, C.A. (2010). Fluid Mechanics and Thermodynamics of Turbomachinery. Butterworth-Heinemann, USA.

DrInG, R. (1984). Blockage in axial compressors. Journal of Engineering for Gas Turbines and Power, 106, 712-714. 
EngedA, A. (1996). The influence of a diffuser width on the unsteady performance of a centrifugal compressor stage. Proceedings of ASME 2002 Joint U.S.European Fluids Engineering Division Conference, Montreal, Quebec, Canada, July 14-18, 2002.

EngedA, A. (1997). Comparative rotating stall and surge characteristic of a centrifugal compressor with three different types of diffusers. Proceedings of ASME 1997 International Gas Turbine and Aeroengine Congress and Exhibition, Orlando, Florida, USA, June 2-5, 1997.

Ernsthausen, W. (1937). The source of propeller noise. NACA, TM 825.

FARAssat, F. (1975). Theory of noise generation from moving bodies with an appliaction to helicopter rotors. tech. rep., NASA, TR R-451.

Farassat, F. (1986). Prediction of advanced propeller noise in the time domain. AIAA, 24, 578-584.

FARAssat, F. (1994). Introduction to genearlized functions with applications in aerodyanmics and aeroacoustics. NASA-TP-3428, corrected April 1996.

FARAssat, F. (2000). The integration of $\delta^{\prime}(f)$ in a multidimensional space. NASA-TP-3428, 230, 460-462.

Farassat, F. (2007). Derivation of Formulations 1 and $1 \mathrm{~A}$ of Farassat. NASA/TM-2007-214853.

Farassat, F. \& Brentner, K.S. (1998). Supersonic quadrupole noise theory of high-speed helicopter rotors. Journal of Sound and Vibration, 218(3), 481500 .

Farassat, F. \& Casper, J. (2012). Broadbandnoiseprediction when turbulencesimulation is available - derivation of formulation $2 \mathrm{~b}$ and its statisticalanalysis. Journal of Sound and Vibration, 331(10), 2203-2208.

Farassat, F. \& Myers, M.K. (1988). Extension of kirchhoffs formula to radiation from moving surfaces. Journal of Sound and Vibration, 123(3), 451-461. 
FfowCS-Williams, J.E. \& HALl, L.H. (1970). Aerodynamic sound generation by turbulent flow in the vicinity of a scattering half plane. Journal of Fluid Mechanics, 40, 657-670.

Ffowcs-Williams, J.E. \& Hawkings, D.L. (1969). Sound generantion by turbulence and surfaces in arbitrary motion. Proceedings of the Royal Society of London, Series A, Mathematical and Physical Sciences, 264(1151), 321342.

Fukano, T. \& JAng, C. (2004). Tip clearance noise of axial flow fans operating at design and off-design condition. Journal of Sound and Vibration, 275, 833853.

Gancedo, M., Guillou, E. \& Gutmark, E. (2014). Bleed slot benefits on turbocharger centrifugal compressor stability. Proceedings of ASME Turbo Expo 2014: Power for Land, Sea and Air, June 16-20, 2014, Düsseldorf, Germany.

Ganz, U.W., Joppa, P.D., Pattern, T.J. \& Scharpf, D.F. (1998). Boeing 18-inch fan rig broadband noise test. Tech. Rep. CR-1998-208704, NASA.

Garrick, I.E. \& Watkins, C.E. (1954). A theoretical study of the effect of forward speed on the free-space sound-pressure field around propellers. NACA Report, 1198.

Gliebe, P., Mani, R., Shin, S., Mitechell, B., Salamah, G. \& Connell, S. (2000). Aeroacoustic prediction codes. NASA/CR-2000-210244.

Goto, T., Онмото, E., Онта, Y. \& Outa, E. (2009). Noise reduction and surge margin improvement using tapered diffuser vane in a centrifugal compressor. Journal of Thermal Science, 19(1), 21-25.

Gutin, L. (1948). On the sound of a rotating airscrew. NACA, TM 1192.

Hah, C., Rabe, D. \& Wadia, A. (2004). Role of tip leakage vortices and passage shock in stall inception in a swept transonic compressor rotor. ASME Turbo Expo 2004, June 14-17, 2004, Vienna, Austria, GT2004-53867. 
Hawthorne, W.R., Wang, C., Tan, C. \& McCune, J. (1984). Theory of blade design for large deflection:Part I - Two-dimensional cascade. Journal of Turbomachinery, 106, 346-353.

Hung, N. (2013). Aero and Vibroacoustics of Automotive Turbochargers. Springer Berlin Heidelberg.

IANNIELlo, S. (1999). Algorithm to integrate the ffowcs williams-hawings equation on supersonic rotating domain. AIAA, 37(9), 1048-1054.

IANNIELlo, S. (2007). New perspectives in the use of the ffowcs williams hawkings equation for aeroacoustic analysis of rotating blades. Journal of Fluid Mechanics, 570, 79-127.

Kameier, F. \& Neise, W. (1997). Rotating blade flow instability as a source of noise in axial turbomachines. Journal of Sound and Vibration, 203, 833-853.

Kay, P. \& Heyes, F. (2014). NT1-10 Quiet Impeller testing. Report No. Dev1392. Napier Turbocharger Technology, Ltd, Lincolnshire.

Khalid, S., Khalsa, A., Waitz, I., Tan, C., Greitzer, E., Cumpsty, N., AdAmCZYK, J. \& MARBLE, F. (1999). End wall blockage in axial compressors. Journal of Turbomachinery, 121, 499-509.

Krain, H. \& Hoffman, B. (2007). Improved high pressure ratio centrifugal compressor. ASME Turbo Expo 2007, Montreal, Canada, GT2007-27100.

Krain, H. \& Hoffman, B. (2008). Flow study of a redesigned high-pressureratio centrifugal compressor. Journal of Propulsion and Power, 24.

Ladson, C.L., Brooks, C.W., Hill, A.S. \& Sproles, D.W. (1996). Program to obtain ordinates for naca airfoils. NASA Technical Memorandum, 4741.

Lamb, H. (1975). Hydrodynamics. Cambridge University Pressure, United Kingdom, 6th edn. 
Leonard, O. \& den Braembussche, R.V. (1992). Design method for subsonic and transonic cascade with prescribed mach number distribution. Journal of Turbomachinery, 114, 553-556.

Lighthill, J. (1967). Introduction to Fourier analysis. Cambridge University Pressure, United Kingdom, 1st edn.

Lighthill, M. (1945). A new method of two dimensional aerodynamics design. Aeronautical Research Council.

Lighthill, M.J. (1952). On sound generated aerodyanmically - 1. genearl theory. Proceedings of the Royal Society of London, Series A, Mathematical and Physical Sciences, 211(1107), 564-587.

Lighthill, M.J. (1954). On sound generated aerodyanmically - 2. turbulence as a source of sound. Proceedings of the Royal Society of London, Series A, Mathematical and Physical Sciences, 222(1148), 1-32.

Lighthill, M.J. (1961). The bakerian lecture: Sound generated aerodynamically. Proceedings of the Royal Society of London, Series A, Mathematical and Physical Sciences, 267(1329), 147-182.

Lighthill, M.J. (1962). The Bakerian Lecture, 1961. Sound Generated Aerodynamcially. Proceedings of the Royal Society of London, Series A, Mathematical and Physical Sciences, 267(1329), 147-182.

LiU, Q., QI, D. \& MaO, Y. (2006). Numerical calculation of centrifugal fan noise. Proc. IMecheE Part C: JMES, 220, 1167-1177.

Lyrintzis, A.S. \& Xue, Y. (1997). Towards a versatile kirchhoffi ${ }^{-}$s method code. AIAA, 35, 198-200.

McAlpine, A., Fisher, M. \& Tester, B. (2006). Buzz-saw noise: A comparison of measurement with prediction. Journal of Sound Vibration, 290, $1202-1233$.

MedD, A. (2002). Enhanced inverse design code and development of design strategies for transonic compressor blading. PhD thesis, Syracuse University. 
Morfey, C.L. (1973). Rotating blades and aerdynamic sound. Journal of Sound and Vibration, 40, 587-317.

Morgans, A.S., Karabasov, S.A., Dowling, A.P. \& Hynes, T.P. (2005). Transonic helicopter noise. AIAA, 43, 1512-1524.

Najafi-Yazzdi, A., Bres, G. \& Mongeau, L. (2010). An acoustic analogy formulation for moving sources in uniformly moving media. Proceedings of the Royal Society of London, Series A, Mathematical and Physical Sciences, 467, 144-165.

Nieuwland, G. (1967). Transonic potential flow around a family of quasielliptical aerofoil sections. National Aerospace Laboratory report NLR-TRT172.

Ohta, Y., Takehara, N., Okutsu, Y. \& Outa, E. (2005). Effects of diffuser vane geometry on interaction noise generated from a centrifgual compressor. Journal of Thermal Science, 14(4), 321-328.

Ohta, Y., Takehara, N., Okutsu, Y. \& Outa, E. (2006). Aerodynamic performance and noise characteristics of a centrifugal compressor with modified vaned diffusers. Journal of Thermal Science, 15(4), 289-295.

Oкамото, H. \& Goto, A. (2009). Design of a propeller fan using 3-D inverse design method and CFD for high efficiency and low aerodynamic noise. Proceedings of FEDSM2009.

Peake, N. \& PARry, A.B. (2012). Modern challenges facing turbomachinery aeroacoustics. Annu. Rev. Fluid Mech., 44, 227-248.

Purcell, T.W. (1988). CFD and transonic helciopter sound. 14th European Rotorcraft Forum, No. 2.

Raitor, T. \& Neise, W. (2008). Sound generation in centrifugal compressors. Journal of Sound and Vibration, 314, 738-756. 
Roois, M. \& MedD, A. (2012). Reformulation of a three-dimensional inverse design method for application in a high-fidelity computational fluid dynamics environment. ASME Turbo Expo 2012: Turbine Technical Conference and Exposition, GT2012-69891.

Sivagnanasundaram, S., Spence, S. \& Early, J. (2012). Map width enhancement technique for a turbocharger compressor. Journal of turbomachinery, 136(6).

Sobieczky, H., Yu, N., Fung, K. \& Seebass, A. (1978). New method for designing shock-free transonic configurations. AIAA, 17(7), 722-729.

Stadler, M., Schmitz, M., Laufer, W. \& Ragg, P. (2013). Inverse aeroacoustic design of axial fans using genetic optimization and the latticeboltzmann method. Journal of Turbomachinery, 136(4).

Stanitz, J. (1953). General design method for three dimensional channels with prescribed velocity along the channel walls. NACA report No.1115.

Stratton, J.A. (1941). Electromagnetic Theory. McGraw-Hill, New York.

Suder, K. (1997). Blockage development in a transonic, axial compressor rotor. NASA Technical Memorandum 113115.

Sun, H. \& LEe, S. (2003). Numerical prediction of centrifugal compressor noise. Journal of Sound and Vibration, 269, 421-430.

Sun, H., Shin, H. \& LeE, S. (2006). Analysis and optimization of aerodynamic noise in a centrifugal compressor. Journal of Sound and Vibration, 289(4), 999-1018.

Tadamasa, A. \& Zangeneh, M. (2011). Numerical prediction of wind turbine noise. Journal of Rewnewable Energy, 36(7), 1902-1912.

Tan, C., Hawthorne, W.R., McCune, J. \& Wang, C. (1984). Theory of blade design for large deflection:Part II - Annular cascades. Journal of Turbomachinery, 106, 354-365. 
Tiow, W., Yiu, K. \& Zangeneh, M. (2002). Application of simulated annealing to inverse design of transonic turbomachinery cascades. Proceedings of the Institution of Mechanical Engineers, Part A: Power and Energy, 216, 59-73.

Tjokroaminata, W. (1992). A design study of radial inflow turbines with splitter blades in three-dimensional flow. Master thesis. Massachusetts Institute of Technology.

Tyler, J.M. \& Sorfin, T.G. (1962). Axial compressor noise studies. Trans Society of Automotive Engineers, 70, 309-322.

Wang, P. \& Zangeneh, M. (2014). Aerodynamic and aeroacoustic optimisaiton for a transonic centrifugal compressor. Proceedings of ASME Turbo Expo 2014: Power for Land, Sea and Air, June 16-20, 2014, Düsseldorf, Germany.

Wang, P. \& Zangeneh, M. (2015). Multi-disciplinary and multi-point optimization of an axial fan for noise and efficiency by $3 \mathrm{~d}$ inverse design method. International Conference on Pumps and Fans(ICPF 2015).

Wang, P., Zangeneh, M., Richards, B., Gray, K., Tran, J. \& AnDAH, A. (2017). Redesign of a compressor stage for a high-performance eletric supercharger in a heavily downsized engine. Proceedings of ASME Turbo Expo 2017: Turbomachinery Technical Conference and Exposition, June 26-30, 2017, Cjarlotte, NC, USA, GT2017-64732.

Wu, C. (1952). A general theory of three-dimensional flow in subsonic and supersonic turbomachines of axial-, radial-, and mixed-flow types. NACA TN 2604 .

Wu, J., Ma, H. \& Zhou, M. (2006). Vorticity and Vortex Dynamics. SpringerVerlag Berlin Heidelberg, Germany, 1st edn.

YANG, L. (1991). A design study of radial inflow turbines in three-dimensional flow. PhD thesis. Massachusetts Institute of Technology.

Yin, J., Li, P. \& PEES, S. (2009). Optimization of turbocharger ported shroud compressor stages. Proceedings of ASME Turbo Expo 2009: Power for Land, Sea, and Air, June 8-12, 2019, Orlando, Florida, USA. 
Zangeneh, M. (1988). Three dimensional design of radial-inflow turbines. University of Cambridge.

ZANGEnEH, M. (1991). A compressible three-dimensional design method for radial and mixed flow turbomachinery blades. International journal of numerical methods in fluids, 13, 599-624.

ZANGENEH, M. (1996). On 3d inverse design of centrifugal compressor impellers with splitter blades. ASME 1998 International Gas Turbine and Aeroengine Congress and Exhibition, Stockholm, Sweden, June 2-5, 1.

Zangeneh, M., Goto, A. \& Harada, H. (1998). On the design criteria for suppression of secondary flows in centrifugal and mixed flow impellers. Journal

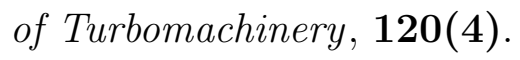

Zangeneh, M., Nikpour, B. \& Watanabe, H. (2010). Development of a high performance centrifugal compressor using a 3d inverse design technique. 9th International Conference on Turbochargers and Turbocharging, 135-145.

Zangeneh, M., Amarel, N., Daneshrhah, K. \& Krain, H. (2011). Optimization of 6.2:1 pressure ratio centrifugal compressor impeller. Proceedings of ASME Turbo Expo 2011: Power for Land, Sea and Air, June 6-11, 2011, Vancouver, Canada.

Zhang, W., Gong, W. \& XI, G. (2010). The influence of the geometry of the leading edge of diffuser vanes on the noise generated by a centrifugal fan. Proc. IMechE Part A: J. Power and Energy, 224, 723-735. 


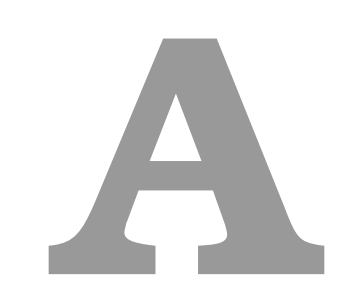

\section{Appendix A: Derivation of Formulation $1 \mathrm{C}$ in terms of source-time derivative}

This appendix provides a detailed derivation of Eq. 4.20 that is for the far-field thickness noise in terms of source-time derivatives $\frac{\partial()}{\partial \tau}$. A similar derivation can be found in Farassat [2007]. One needs to move the observer-time derivative in Eq. 4.19 into the integral Leibnitz rule of differentiation under an integral sign, which is

$$
F(x)=\int_{a(x)}^{b(x)} f(x, t) d t
$$




$$
\frac{d F(x)}{d x}=f(x, b(x)) b^{\prime}(x)-f(x, a(x)) a^{\prime}(x)+\int_{a(x)}^{b(x)} \frac{\partial f(x, t)}{\partial x} d t
$$

Consider a generic integral,

$$
I_{Q}=\frac{\partial}{\partial t} \int_{-\infty}^{t} \int_{\mathbb{R}^{3}} Q(\boldsymbol{y}, \tau) \delta(f) \frac{\delta(g)}{r^{*}} d \boldsymbol{y} d \tau
$$

in which $f=f(\boldsymbol{y}, \tau)$ and $g=\tau-t+r / c$ Using the Leibnitz rule stated above and taking the $\frac{\partial}{\partial t}$ into the integral yields,

$$
I_{Q}=\underbrace{\int_{\mathbb{R}^{3}} Q(\boldsymbol{y}, t) \delta(f(\boldsymbol{y}, t)) \frac{\delta(r / c)}{r^{*}} d \boldsymbol{y}}_{I_{1}}+\underbrace{\int_{-\infty}^{t} \int_{\mathbb{R}^{3}} Q(\boldsymbol{y}, \tau) \delta(f) \frac{\partial}{\partial t}\left(\frac{\delta(g)}{r^{*}}\right) d \boldsymbol{y} d \tau}_{I_{2}}
$$

It will be shown that the first integral $I_{1}$ in the LHS of Eq. A.4 vanishes to zero, and this is proven below.

Recall Eq. 3.34,

$$
r=\frac{-M_{0}\left(x_{3}-y_{3}\right)+r^{*}}{\beta^{2}},
$$

which can be re-expressed as,

$$
r=\frac{r^{*}}{\beta^{2}}\left(1-\frac{M_{0}\left(x_{3}-y_{3}\right)}{r^{*}}\right)
$$

One may convert the Cartesian coordinate $\left(y_{1}, y_{2}, y_{3}\right)$ into Spherical coordinate $\left(r^{*}, \phi, \theta\right)$, then

$$
\cos \theta=\frac{M_{0}\left(x_{3}-y_{3}\right)}{r^{*}}
$$

Subsequently,

$$
\delta(r / c)=c \delta(r)=c \delta\left[\frac{r^{*}}{\beta^{2}}\left(1-M_{0} \cos \theta\right)\right]=\frac{c\left(1-M_{0}\right) \cos \theta}{\beta^{2}} \delta\left(r^{*}\right) .
$$


Inserting Eq. A.7 into $I_{1}$ and using the spherical coordinate system gives,

$$
I_{1}=\int_{0}^{\infty} \int_{0}^{\pi} \int_{0}^{2 \pi} \frac{c\left(1-M_{0}\right) \cos \theta}{\beta^{2}} Q(\boldsymbol{y}, t) \delta(f(\boldsymbol{y}, t)) r^{*} \delta\left(r^{*}\right) d r^{*} \sin \theta d \phi d \theta
$$

which can be re-written as

$$
\begin{aligned}
I_{1}= & \lim _{\epsilon \rightarrow 0} \int_{0}^{\infty} \int_{0}^{\pi} \int_{0}^{2 \pi} \frac{c\left(1-M_{0}\right) \cos \theta}{\beta^{2}} Q(\boldsymbol{y}, t) \delta\left(f\left(r^{*}, \phi, \theta\right)\right) \\
& \times r^{*} \delta\left(r^{*}-\epsilon\right) d r^{*} \sin \theta d \phi d \theta,
\end{aligned}
$$

and since $r^{*} \delta\left(r^{*}-\epsilon\right)=\epsilon \delta\left(\epsilon-r^{*}\right)$, then Eq. A.9 becomes

$$
\begin{aligned}
I_{1}= & \lim _{\epsilon \rightarrow 0}\left\{\epsilon \left[\int_{0}^{\infty} \int_{0}^{\pi} \int_{0}^{2 \pi} \frac{c\left(1-M_{0}\right) \cos \theta}{\beta^{2}} Q(\boldsymbol{y}, t) \delta\left(f\left(r^{*}, \phi, \theta\right)\right)\right.\right. \\
& \left.\left.\times \epsilon \delta\left(\epsilon-r^{*}\right) d r^{*} \sin \theta d \phi d \theta\right]\right\}
\end{aligned}
$$

which vanishes as $\epsilon \rightarrow 0$. Thus,

$$
I_{Q}=\int_{-\infty}^{t} \int_{\mathbb{R}^{3}} Q(\boldsymbol{y}, \tau) \delta(f) \frac{\partial}{\partial t}\left(\frac{\delta(g)}{r^{*}}\right) d \boldsymbol{y} d \tau
$$

Subsequently, one can take the observer temporal derivatives in Eq. 4.18 inside 
the integral leading to,

$$
\begin{aligned}
4 \pi p_{T}^{\prime}(\mathbf{x}, t)= & \int_{-\infty}^{t} \int_{V} \frac{\partial}{\partial t}\left[Q_{i} n_{i} \delta(f) \frac{\delta(g)}{r^{*}} d \tau\right] d^{3} \mathbf{y} \\
& -M_{0} \int_{-\infty}^{t} \int_{V} \frac{\partial}{\partial t}\left[Q_{j} n_{j} \delta(f) \frac{\tilde{r}_{3} \delta(g)}{r^{*}} d \tau\right] d^{3} \mathbf{y} \\
& -U_{0} \int_{-\infty}^{t} \int_{V} Q_{j} n_{j} \delta(f) \frac{\tilde{r}_{3}^{*} \delta(g)}{r^{* 2}} d^{3} \mathbf{y} d \tau \\
= & \int_{f=0, g=0} \frac{\partial}{\partial t}\left[\frac{Q_{i} n_{i}}{r^{*}\left(1-M_{R}\right)}\right] d^{3} \mathbf{y} \\
& -M_{0} \int_{f=0, g=0} \frac{\partial}{\partial t}\left[\frac{Q_{j} n_{j} \tilde{r}_{3}^{*}}{r^{*}\left(1-M_{R}\right)}\right] d^{3} \mathbf{y} \\
& -U_{0} \int_{f=0, g=0} \frac{Q_{j} n_{j} \tilde{r}_{3}^{*}}{r^{* 2}\left(1-M_{R}\right)} d^{3} \mathbf{y},
\end{aligned}
$$

where the observer-time derivative can be transformed to source-time derivative by,

$$
\frac{\partial}{\partial t}=\frac{1}{1-M_{R}} \frac{\partial}{\partial \tau}
$$

Substituting the above identity into Eq. A.12, the final integral representation for thickness noise (Eq. 4.20) can be obtained. The loading noise (Eq. 4.23) can be deduced in a similar way. 